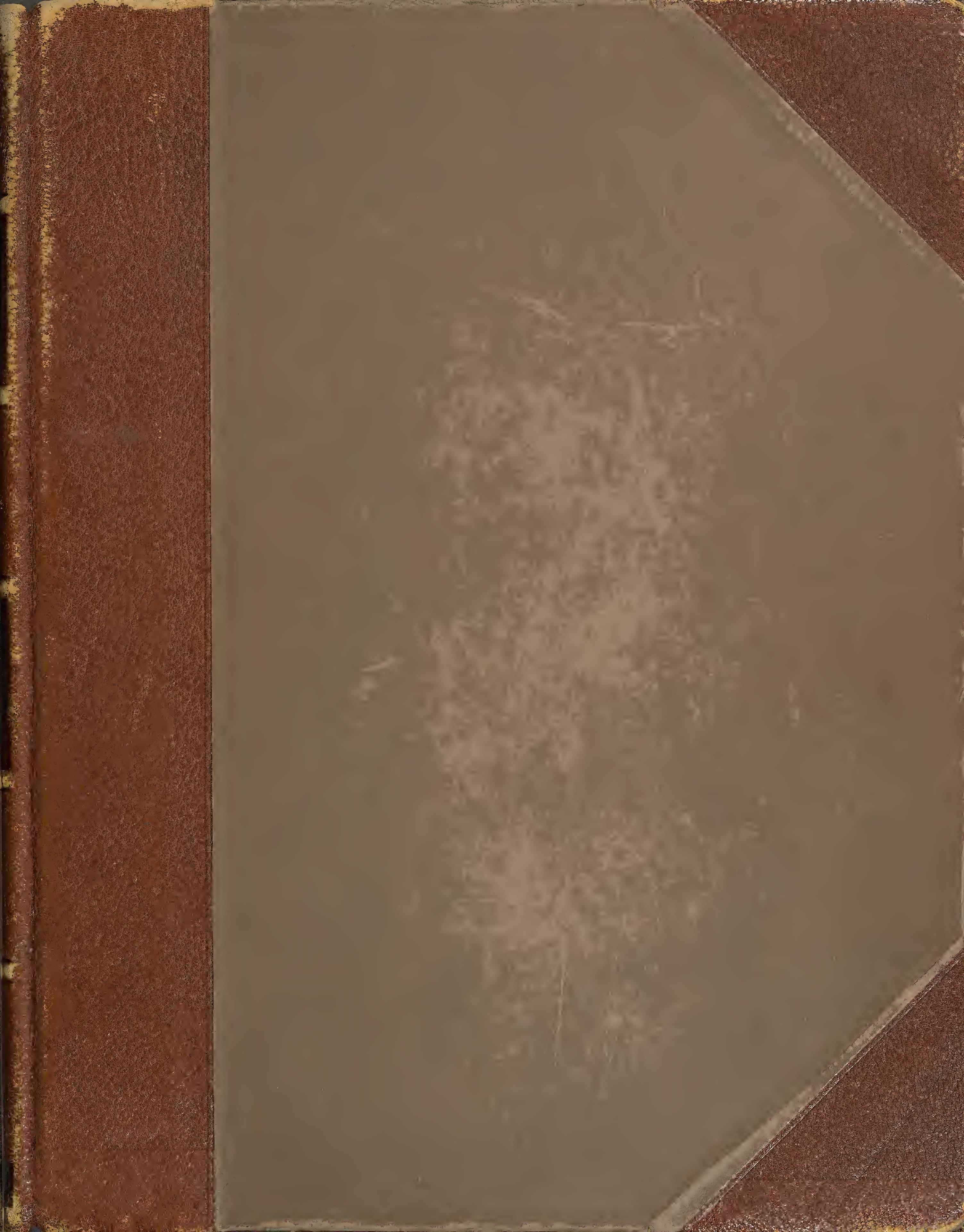










FA GSIMILE

OF THE

WASHINGTON MANUSCRIPT OF THE

MINOR PROPHETS IN THE FREER COLLECTION

THE BERLIN FRAGMENT OF GENESIS 
EDITION OF 400 COPIES

This is copy No. /

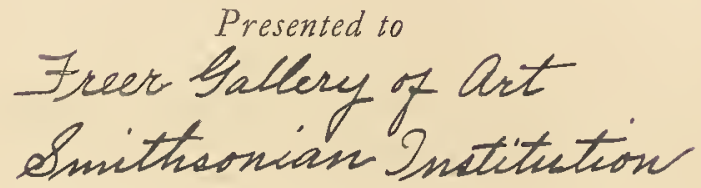

By the University of Michigan 


\title{
FACSIMILE
}

OF THE

\section{WASHINGTON MANUSCRIPT \\ OF THE}

MINOR PROPHETS

IN THE FREER COLLECTION

AND THE

\section{BERLIN FRAGMENT OF GENESIS}

\author{
WITH AN INTRODUCTION BY \\ HENRY A. SANDERS
}

Finn Frbor, SIDichigan

THE UNIVERSITY OF MICHIGAN

1927

Freer Gallery of Ant

Washington, $D, C$. 



\section{CONTENTS}

INTRODUCTION

I. The Papyrus of the Minor Prophets . . . . . . . . . . . . . . . . . vii

- II. The Berlim Fragment of Genesis . . . . . . . . . . . . . . . . . . . . . ix

PART I. THE FACSIMILE OF THE WASHINGTON MANUSCRIPT OF THE MINOR PROPHETS

Fragments OF Hosea . . . . . . . . . . . . . . . . . . . . . . . . . . I

Amos . . . . . . . . . . . . . . . . . . . . . . . . . . 3

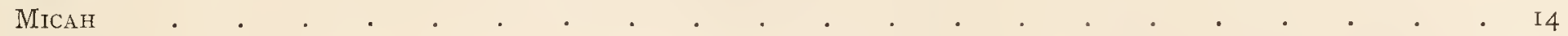

Joel • • • • •

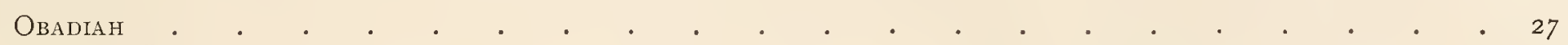

JONAH $\cdot$ •

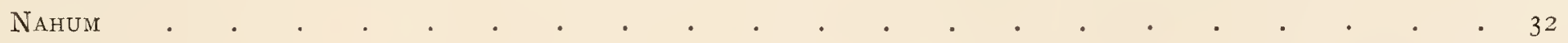

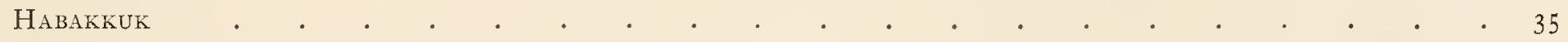

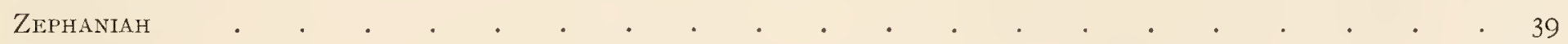

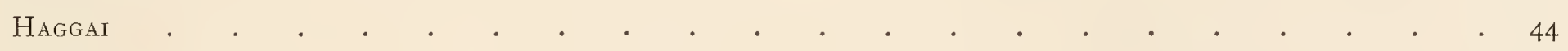

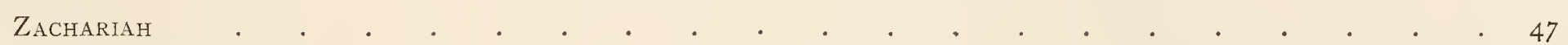

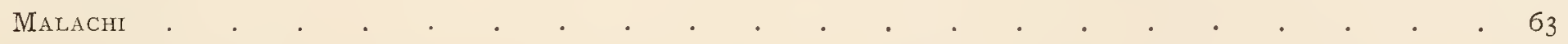

Unplaced Fragmetts

PART II. THE FACSIMILE OF THE BERLIN FRAGMENT OF GENESIS

Genesis, Chapter i, i6 (beginning)

Chapter xxxv, 8 (end) 



\section{NTRODUCTION}

\section{PAPYRUS OF THE MINOR PROPHETS}

$\mathrm{T}$

HE papyrus of the Minor Prophets, of which the Facsimile Edition forms the first part of this volume, is Greek Manuscript V of the Freer Gallery in the Smithsonian Institution, Washington, D. C. A full discussion of the manuscript appears simultaneously in the University of Michigan Studies, Humanistic Series, Vol. XXI, Part I. For the convenience of users of the facsimile a brief summary is appended here.

The whole papyrus was written by the same scribe in a semi-cursive hand, about the middle of the third century, in Egypt. From various considerations it is inferred that the manuscript was once in use in Sahidic or Achmimic territory. It was carefully corrected by a contemporary scribe, the $\delta\llcorner\rho \rho \theta \omega \tau \eta \dot{s}$, and there are many other corrections scattered through the text. All the approximately contemporary corrections in similar ink have been assigned to the second hand, and those in blacker ink and more crudely written, to the third hand, though it is recognized that the work of more than one corrector is included under each hand. In addition to these corrections in semi-cursive or uncial there are a few marginal notes in pure cursive and many more in Coptic. Most of these are too fragmentary to be read with certainty.

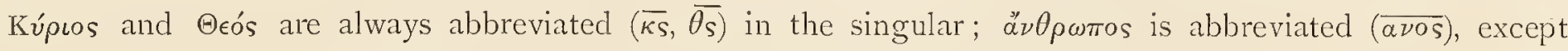

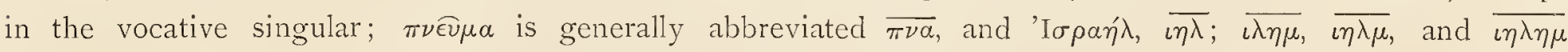

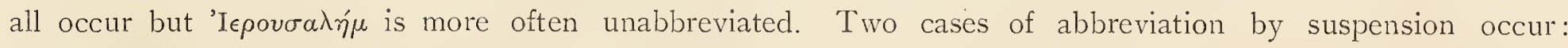

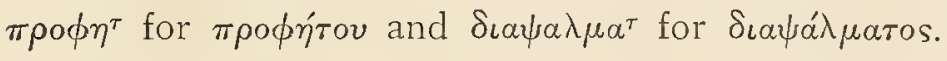

The common punctuation is a single dot in high position; rarely it occurs in low position. Two dots in the form of a colon occur a few times as a major division. The coronis is found at the end of each Prophet, and a simpler form of the paragraph mark occurs rarely elsewhere.

A large number of accents are read with certainty, but not all are from the original scribe. In general they conform to the system still in use. The rough breathing is found in the form $\llcorner$, or rarely $r$; the smooth breathing - is rare. Double dots occur frequently over initial $\iota$ and $v$, rarely when not initial. An apostrophe appears often after foreign names ending in a consonant and rarely at other word ends. 
The manuscript was in book form and consisted of a single quire. Originally there were probably 48 leaves, but 9 leaves at the end were either left vacant or used for another work. The original size of the double sheets of papyrus used in making the quire must have been about II $\frac{3}{4}$ by $\mathrm{I} 3 \frac{1}{2}$ inches. The facsimile shows the exact size at present.

All unplaced fragments of the manuscript or binding, as well as three fragments tentatively placed in the earlier chapters of Hosea, are united on pages I and 2 ; after these follow, pages 3 to 68 , the 33 consecutive leaves of the manuscript as united. On the last two unnumbered pages are reproduced a number of very dim fragments, which seem to come from a different manuscript and to have been written by a different hand. As they were found mixed with the fragments of the Minor Prophets they are reproduced here, though I have not been able to identify them.

The photographing for this edition was done by Mr. George R. Swain, photographer for the University of Michigan. The plates were made by the photo-gelatine process of the Heliotype Company of Boston, Mass.

The manuscript is rather dim and discolored and in places is almost illegible. The utmost pains were taken to secure the best possible photographs, yet at times one can see letters a little more clearly in the original. The pages of the facsimile are entirely free from retouching. 


\section{THE BERLIN FRAGMENT OF GENESIS}

$\mathrm{T}$

HE second part of this facsimile volume contains a large papyrus fragment of Genesis, now in the Staatsbibliothek in Berlin, where it bears the number Graec. Fol. 66, I, II. In Rahlfs' list, Septuaginta-Unternehmens, Vol. II, it has the number 91 I. As a full introduction, a reprint of the text, and explanatory notes appear simultaneously in Vol. XXI, Part II, of the University of Michigan Studies, Humanistic Series, only a brief statement of those matters necessary for understanding the facsimile is added here.

The whole papyrus was written by one scribe in a cursive hand in the latter part of the third century A.D. in Egypt. Corrections are not numerous, and seem to have been made by the same hand, or at least in the same ink.

Of the common Church abbreviations only $\overline{\kappa_{s}}$ and $\overline{\theta_{s}}$ occur, and these in the singular only. Rarely

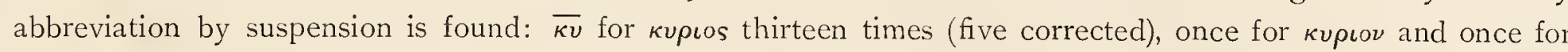
$\kappa \nu \rho \iota \omega$. Other cases noted are $\nu \nu \bar{\kappa}$ for $\nu \nu \kappa \tau \alpha, \bar{\theta}$ for $\theta \eta \sigma \omega, \epsilon \pi \circ \rho \bar{\epsilon}$ for $\epsilon \pi \circ \rho \epsilon u \theta \eta$, and $\bar{\delta}$ for $\iota \delta o v$. A simple abbreviation stroke marks the omission of a single letter, medial as well as final. In order of frequency the omitted letters are $\nu, \sigma, \iota, v, \rho, a, \eta, \mu$, and $\omega$. The same stroke often seems to represent a rough breathing or to replace the curved stroke as an apostrophe. Other instances of a like stroke are best explained as accent, emphasis, or even deletion.

There is no punctuation and but few paragraph marks. Line fillers $>>$ are rarely used. Two dots are often placed over iota, when initial or preceded by another vowel with which it does not form a diphthong. Less often the dieresis appears above upsilon.

The papyrus, written on both sides, formed a single quire with double leaves sewed through the middle between the encroaching columns of writing. Originally there were sixteen double sheets, fourteen by ten inches, of which the outer one, which served chiefly as cover, has been lost. All written pages were numbered in Coptic fashion, beginning on the inside of the first leaf. Three of these page numbers are preserved, in whole or in part.

The text includes Genesis, I, I6 to 35,8 , but no leaf is perfect and some are very fragmentary. The excellent photographs for the facsimile were furnished by the Staatsbibliothek of Berlin and the plates were made by the photo-gelatine process of the Heliotype Company of Boston, Mass. The pages of the facsimile are entirely free from retouching and, in painting out lacunae and margins, the utmost pains were taken not to obscure any letter. 

PART I

THE FAGSIMILE OF THE WASHINGTON MANUSGRIPT

$\mathrm{OF}$

THE MINOR PROPHETS 



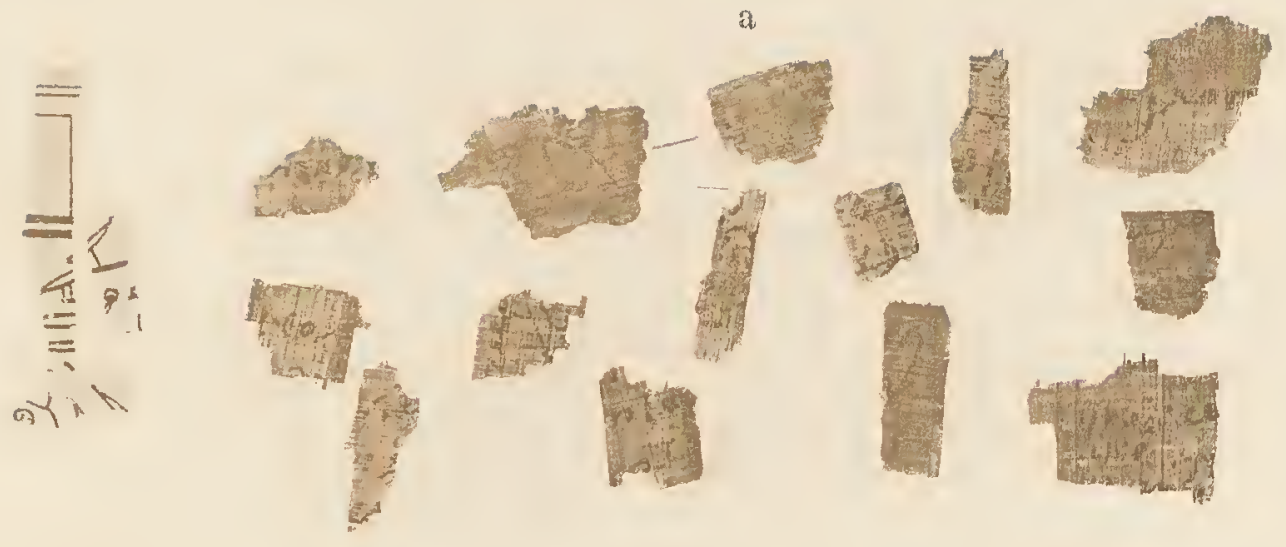

b
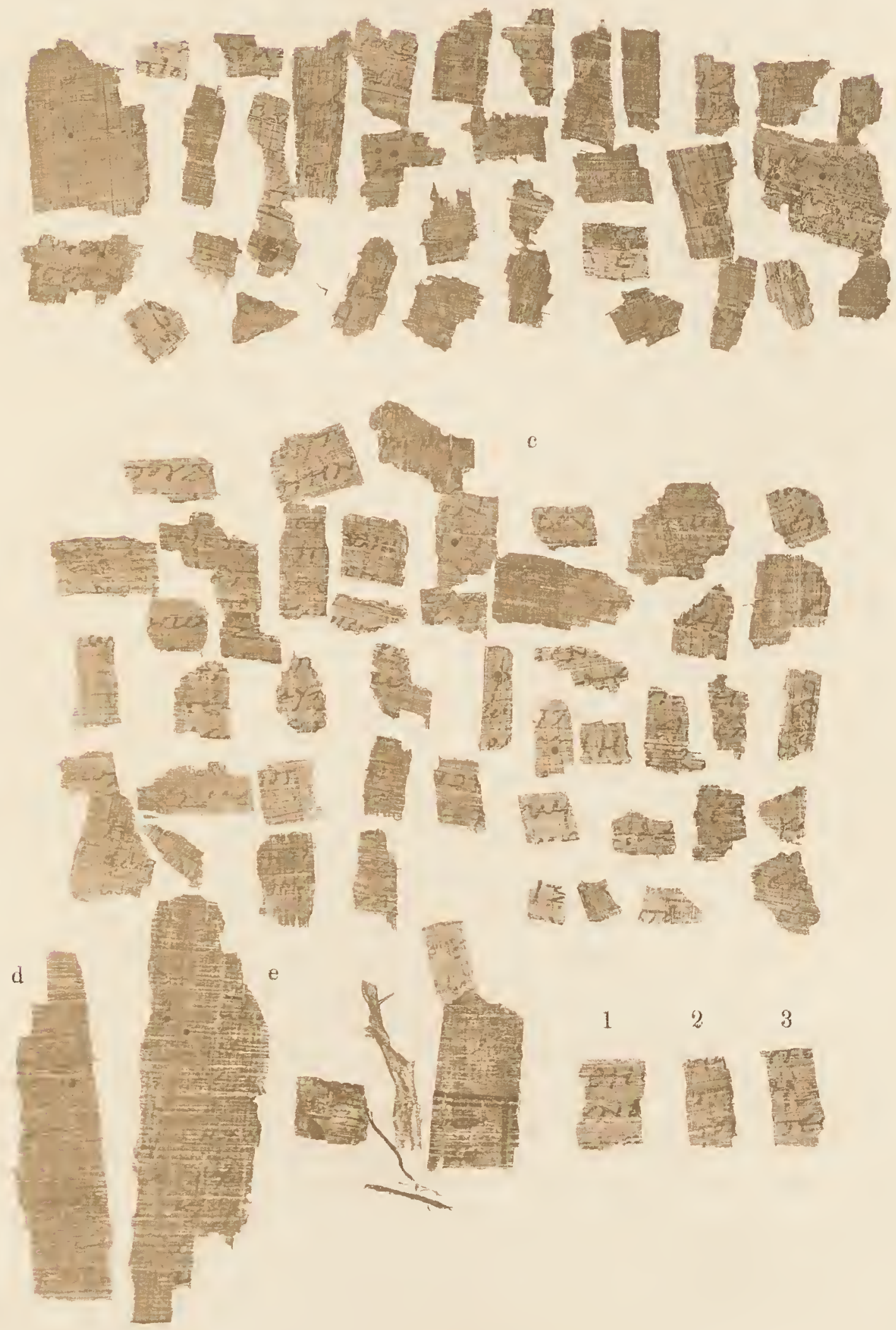

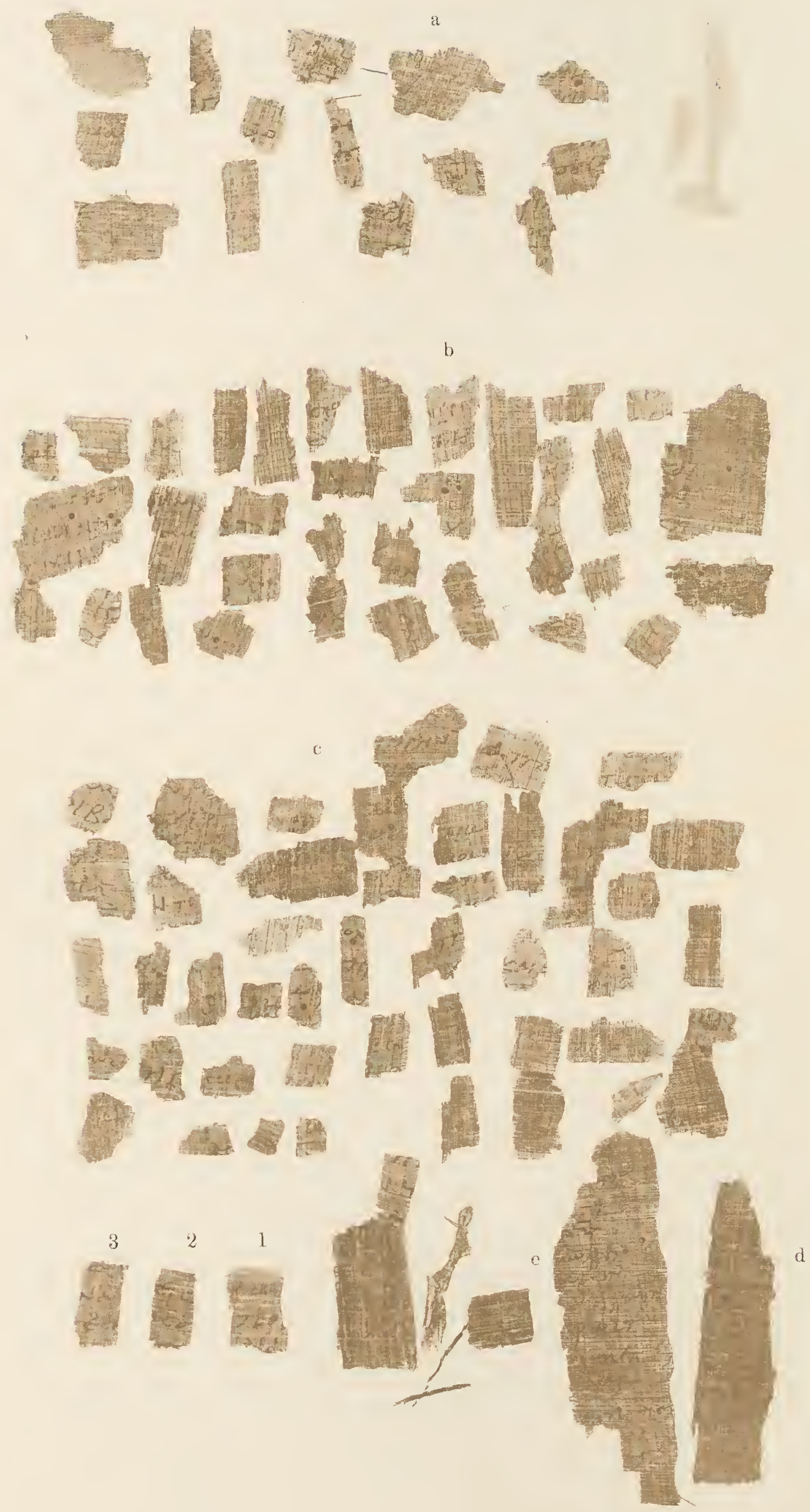

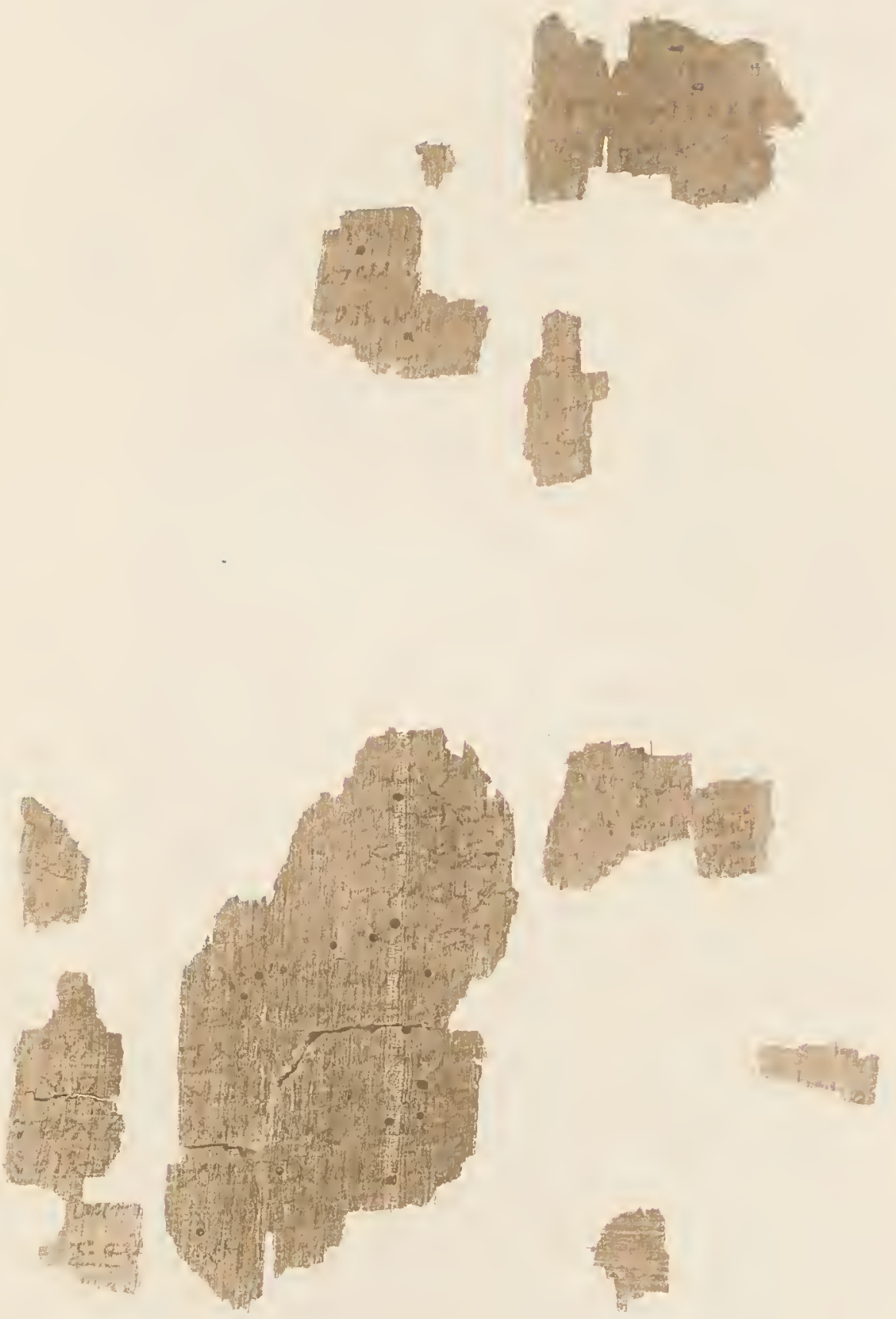

Hoseas xiv, $\tilde{\tau}-A$ mos $i, j$. 

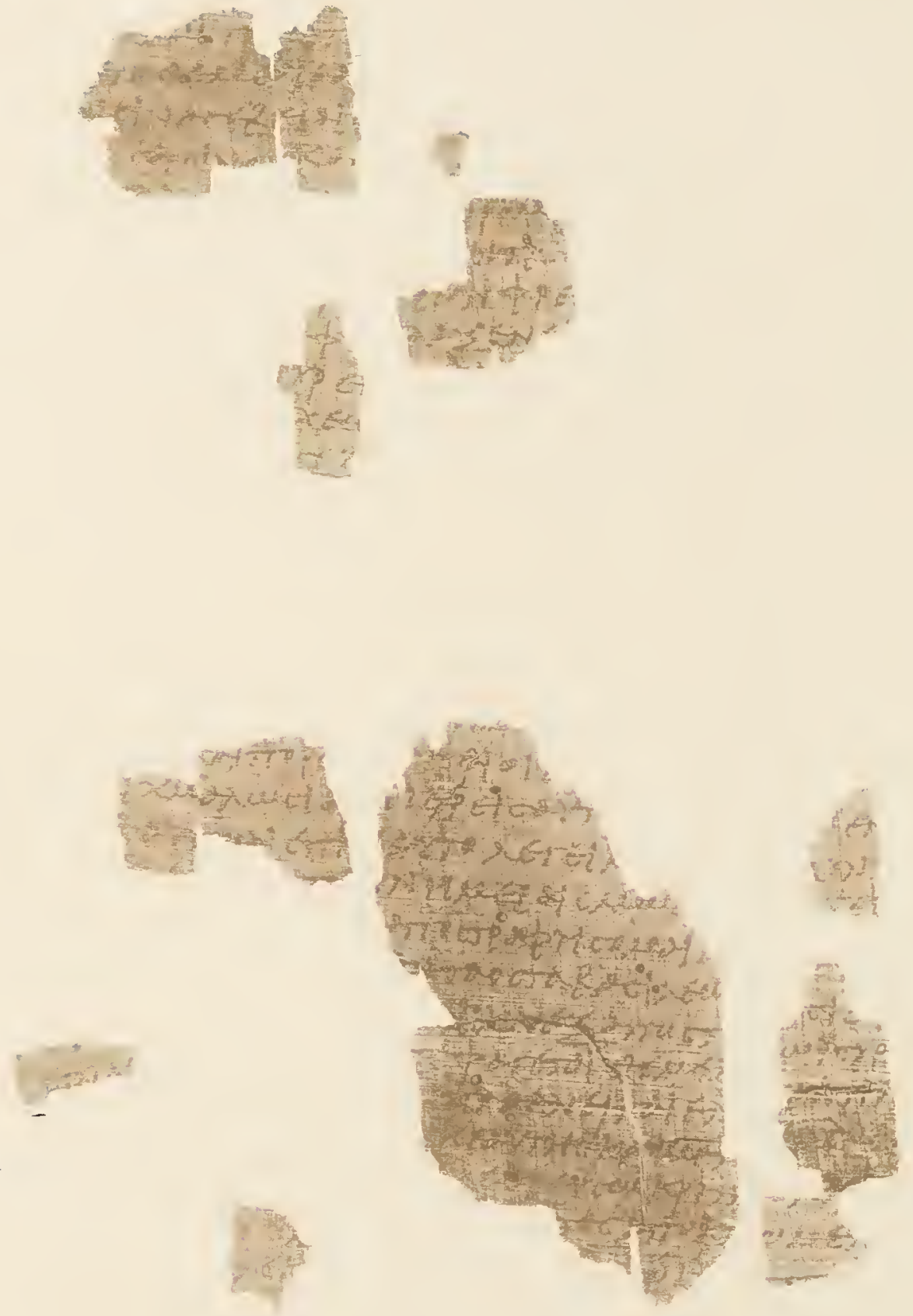


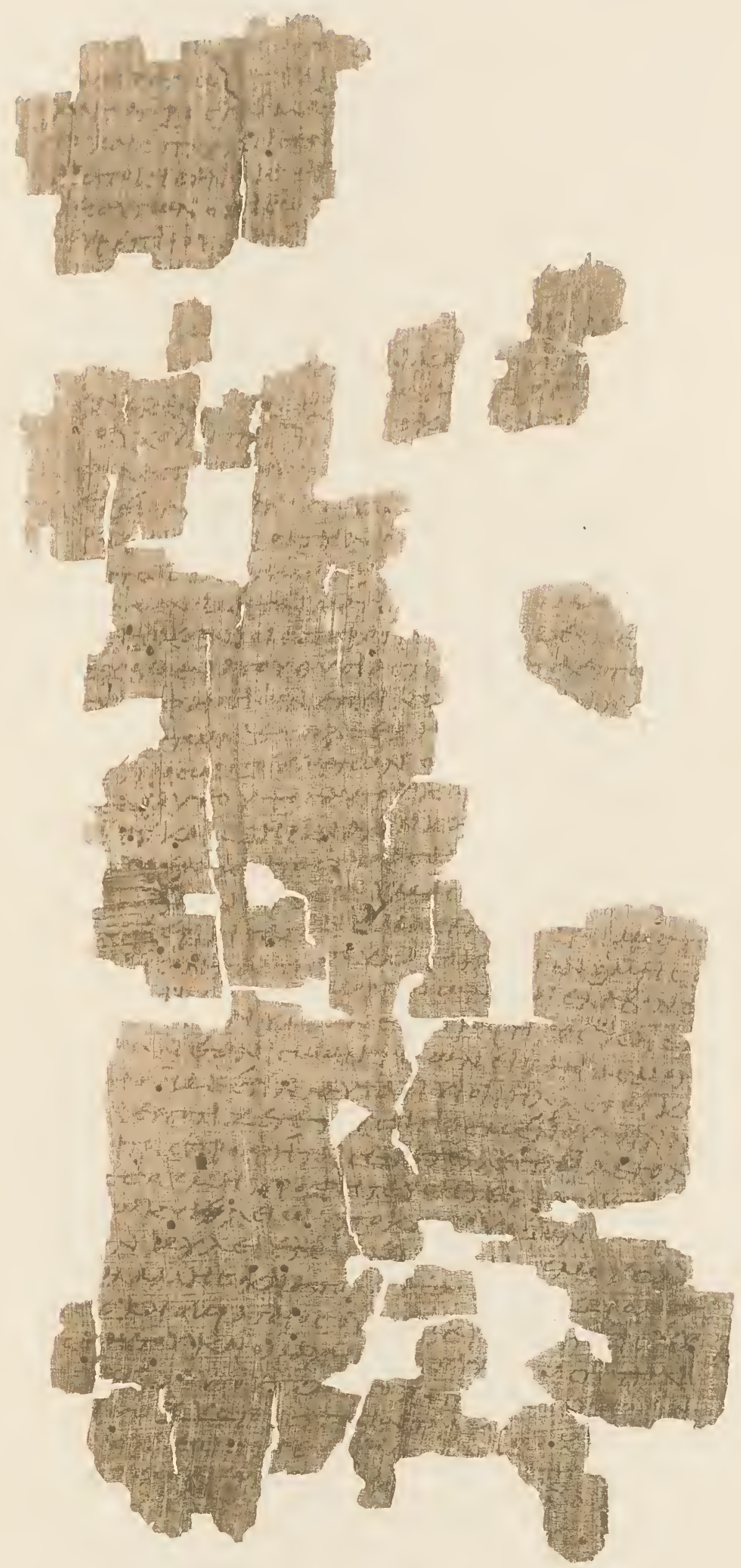




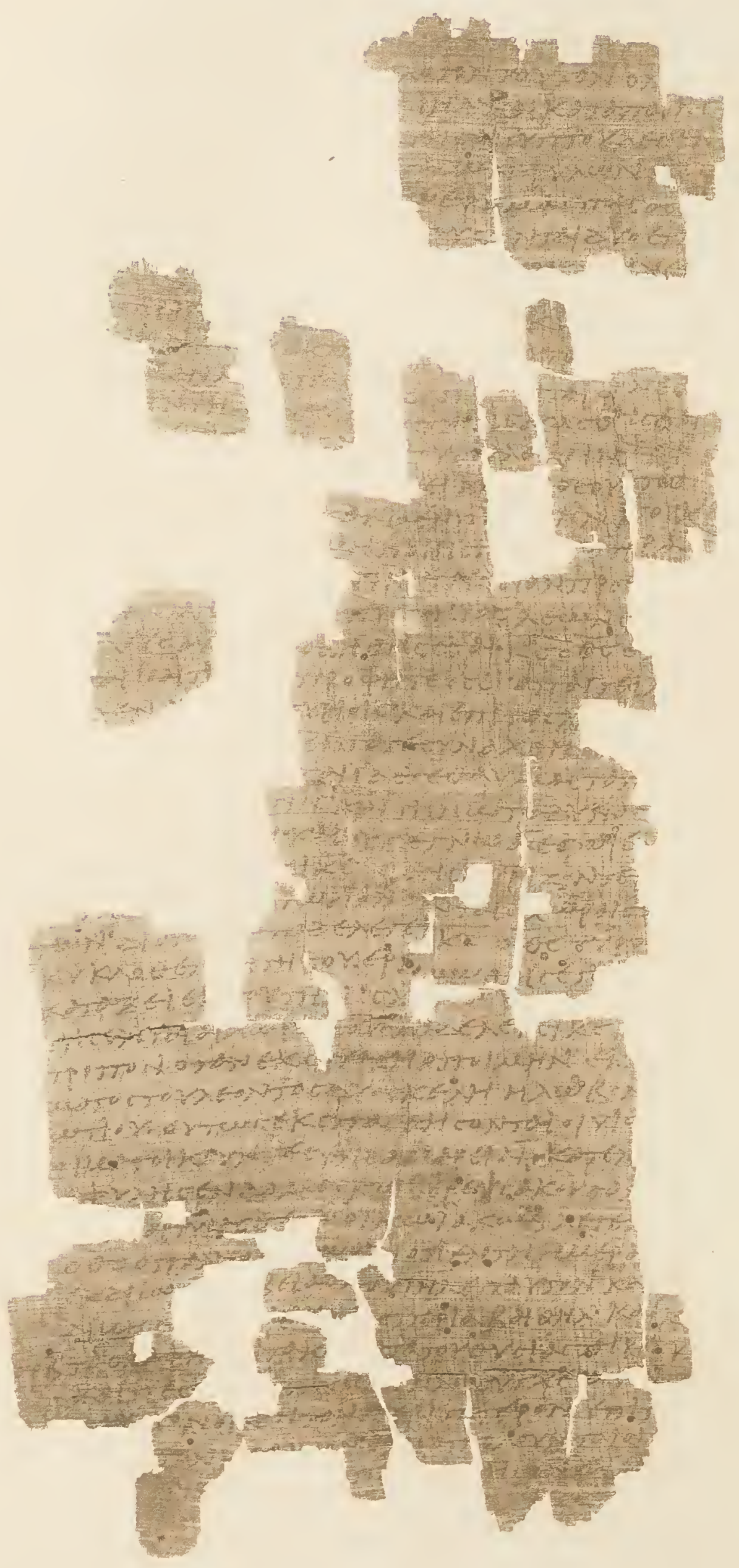

Axos ii, 16-iv, 1. 

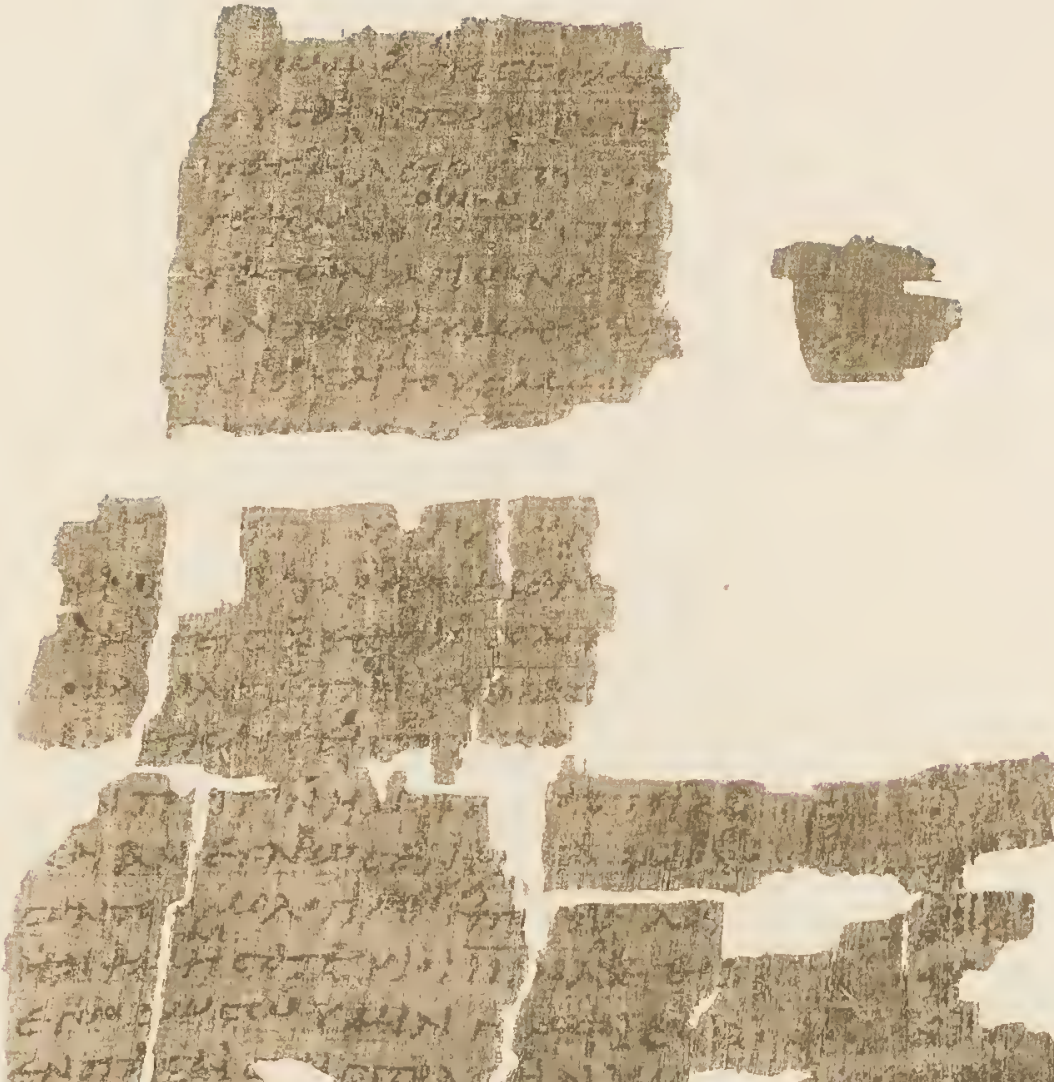

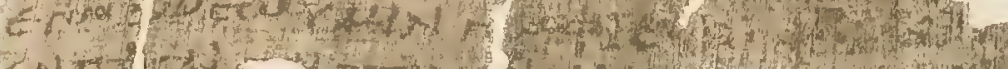

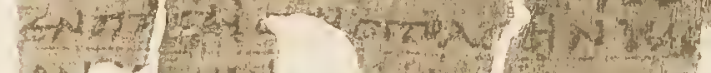

an'

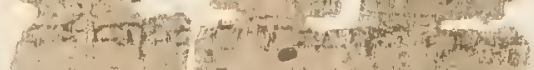

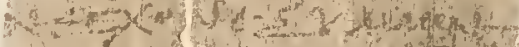

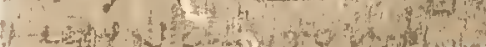
-
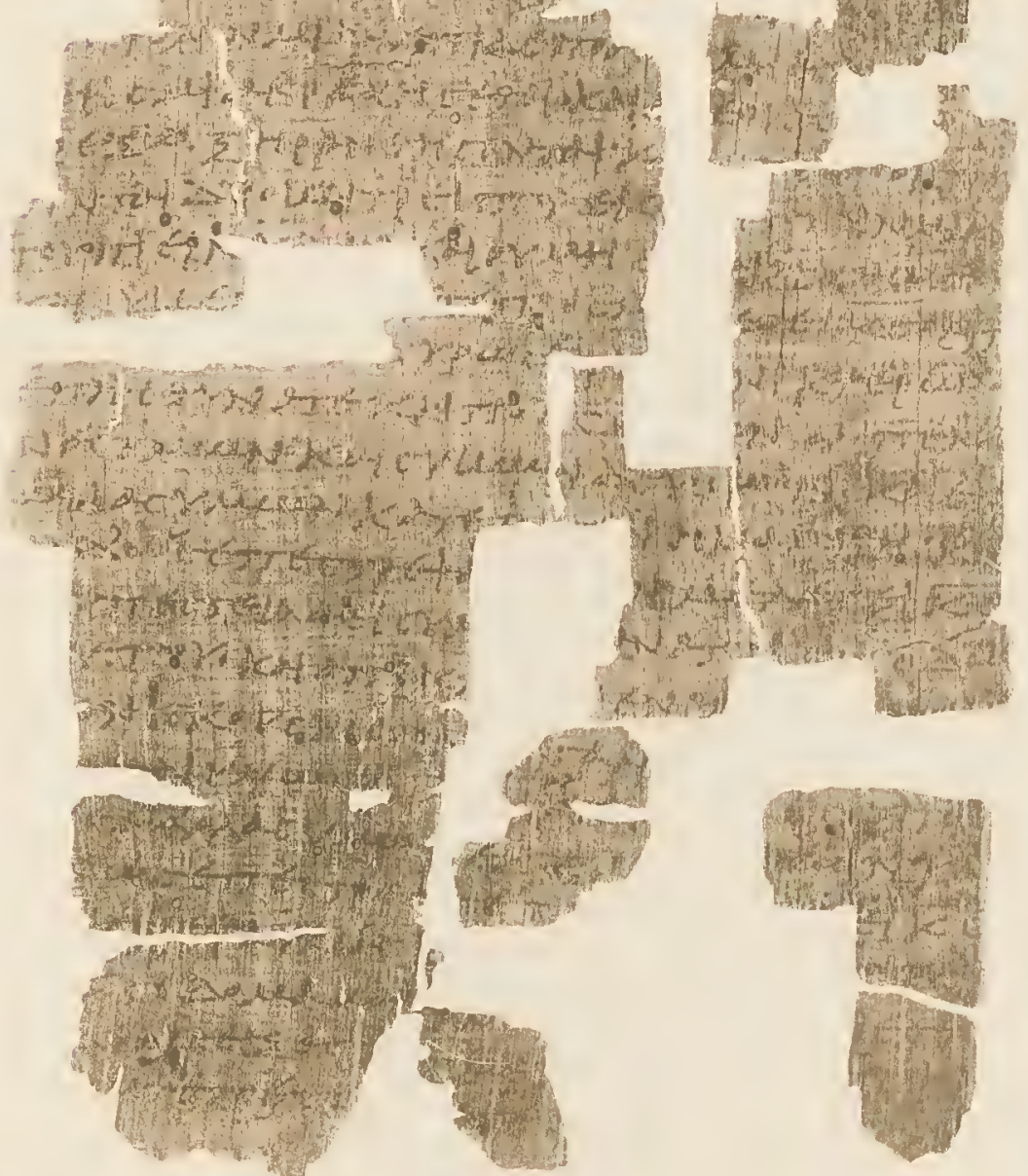


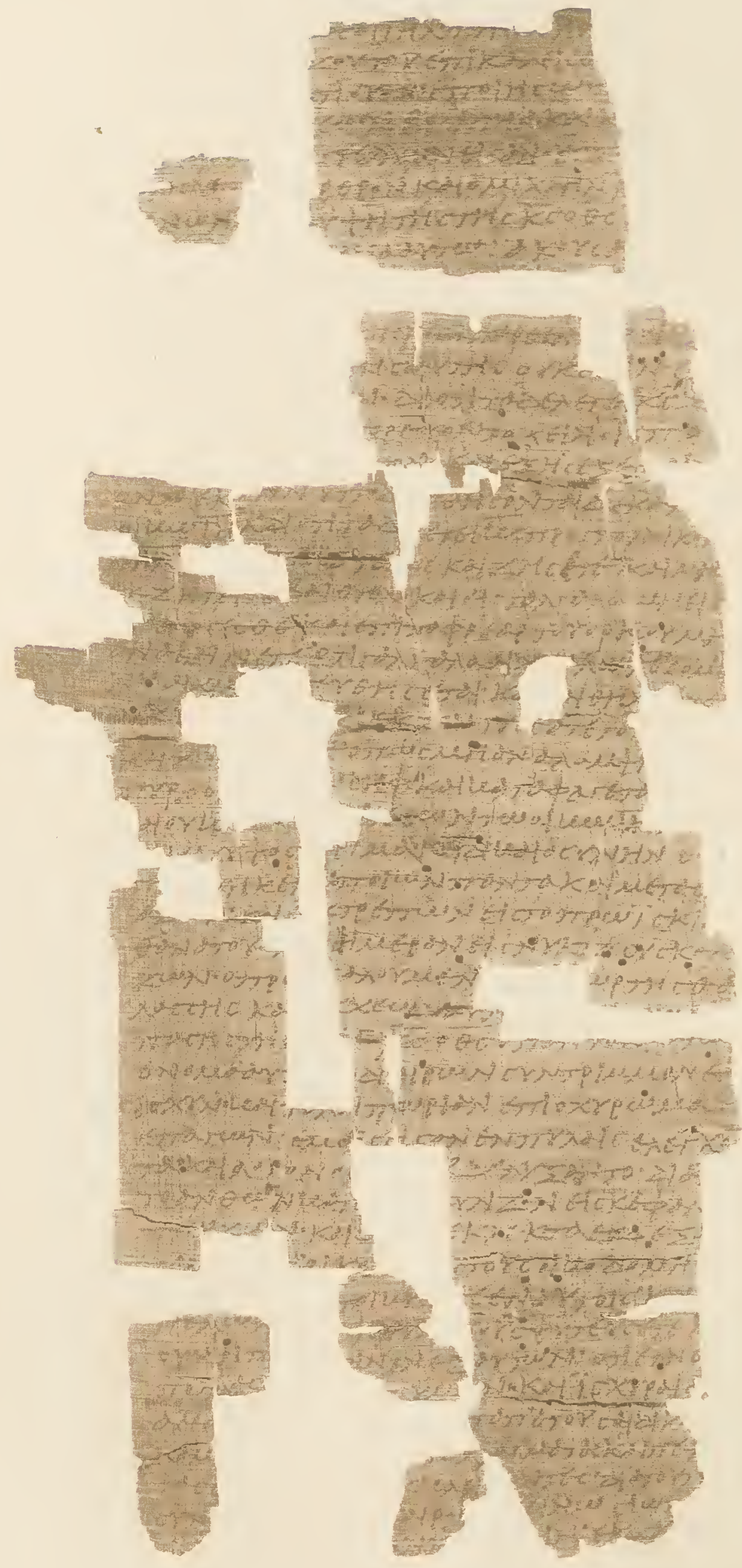



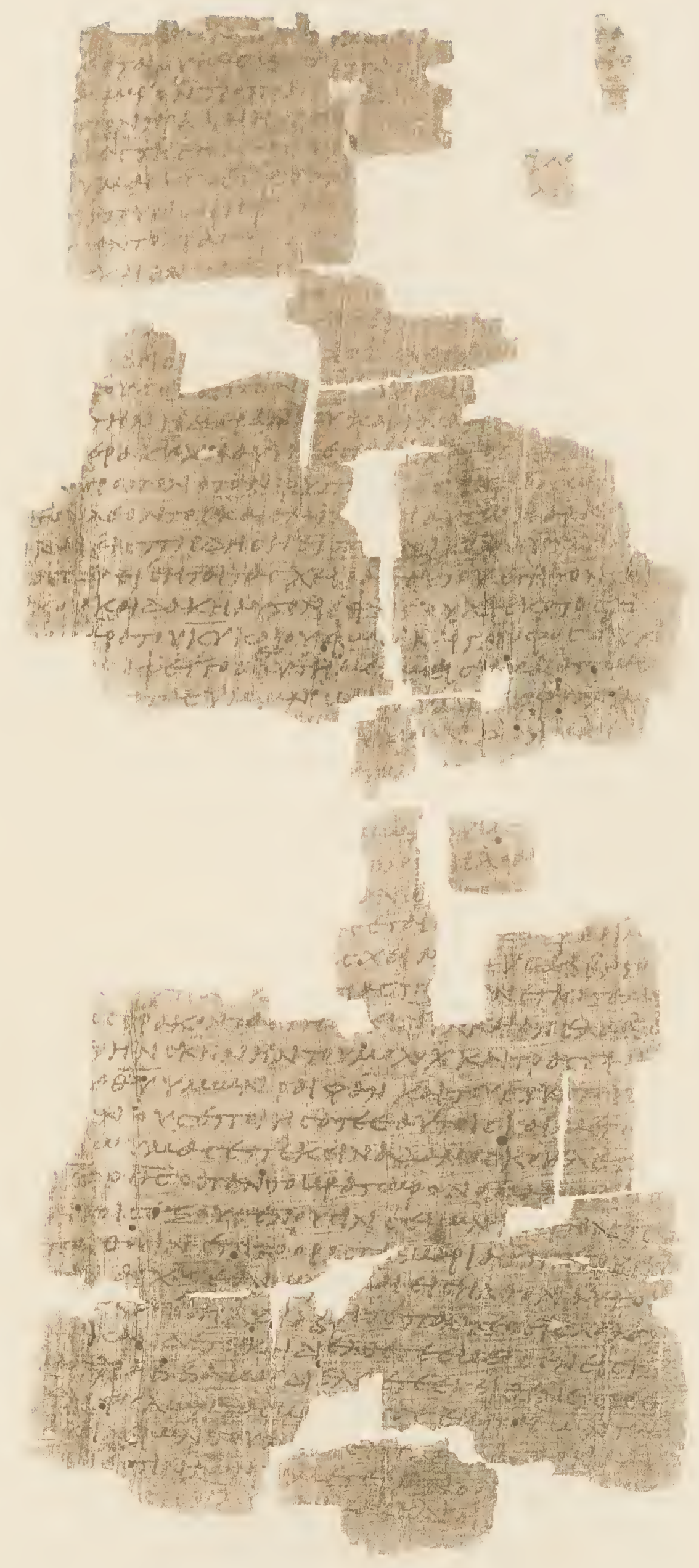

Amos v, 14-vi, 3. 


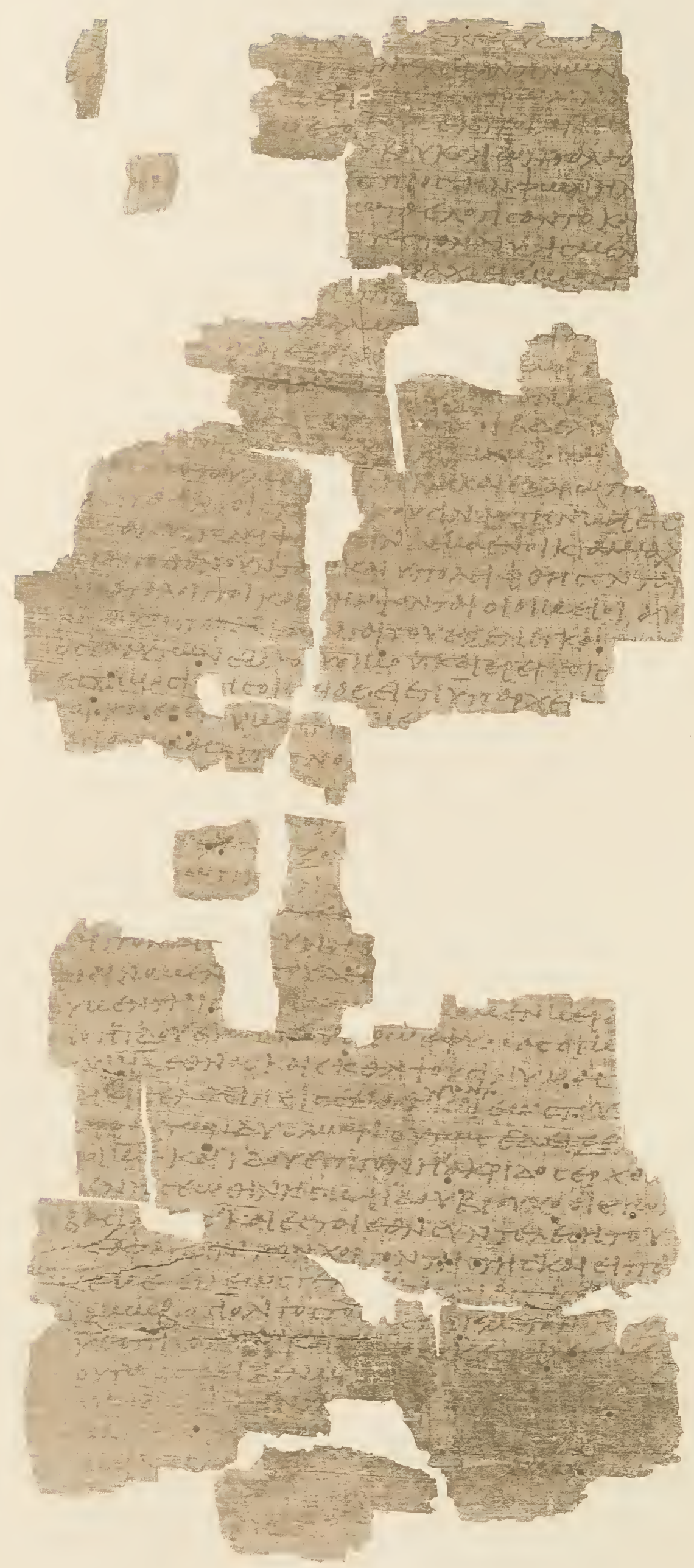

Amos vi, 3 - vii, 5 


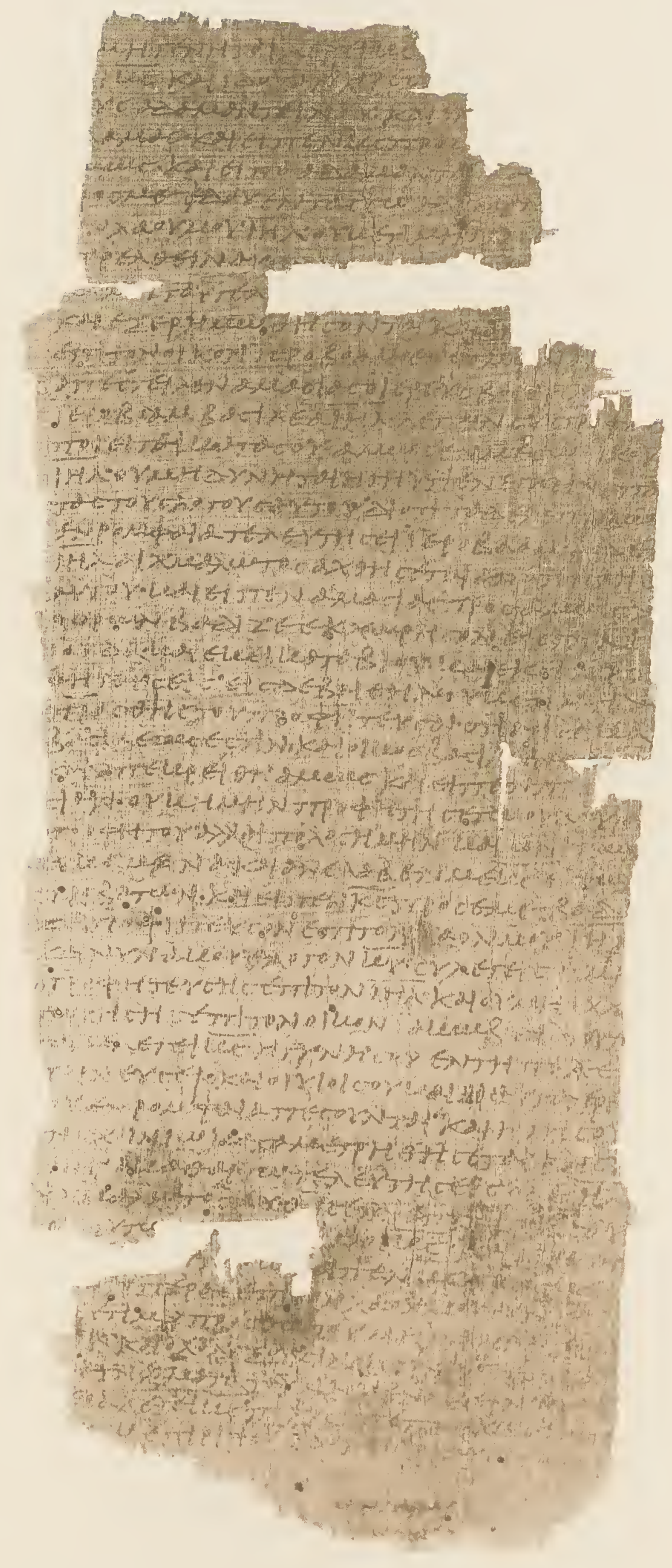

A Mos vii, 5-viii, 4 . 


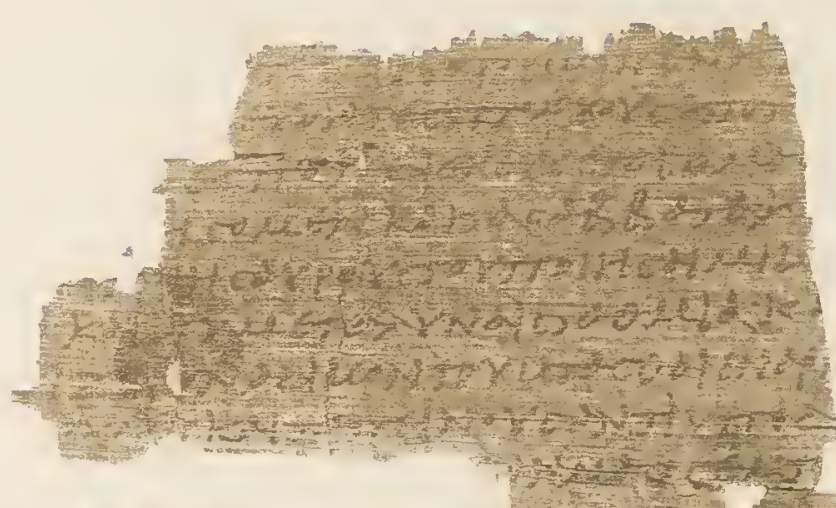

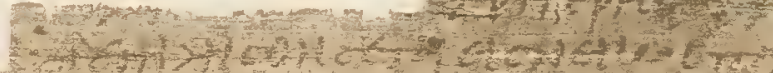

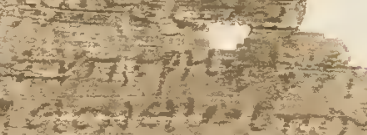

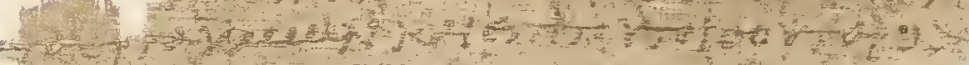

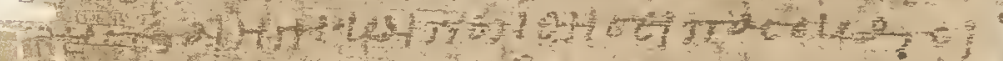
11. why

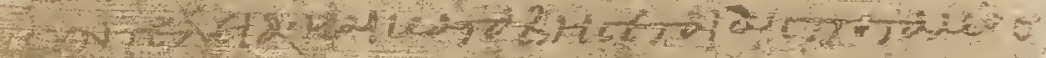

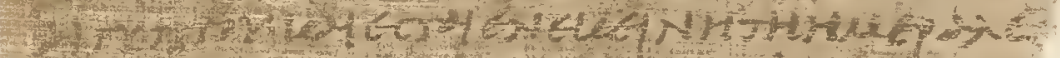

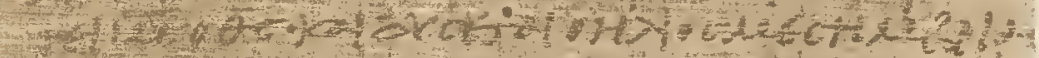

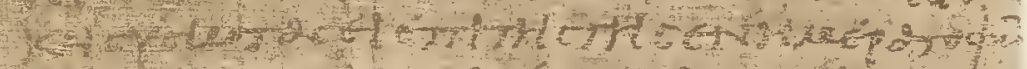

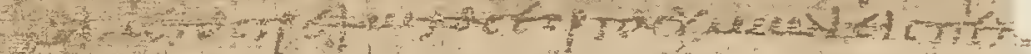

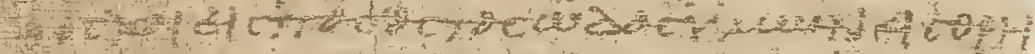

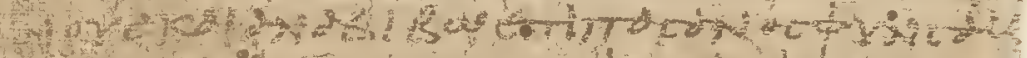

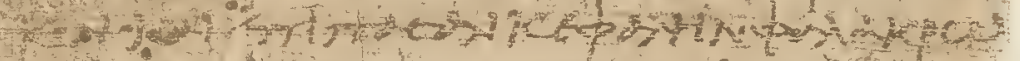

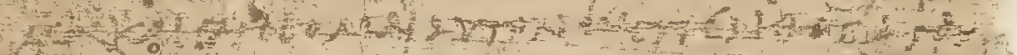

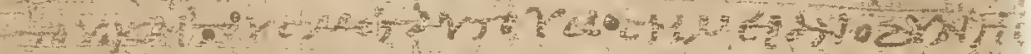

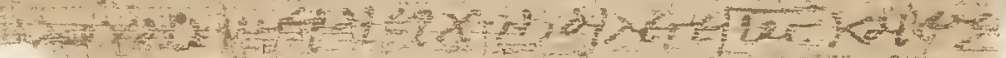

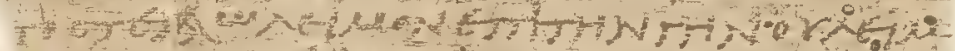

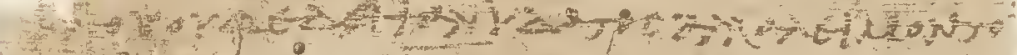

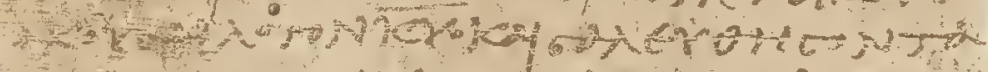

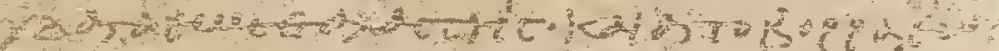

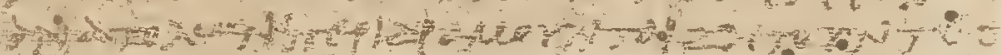
Thx Hacer

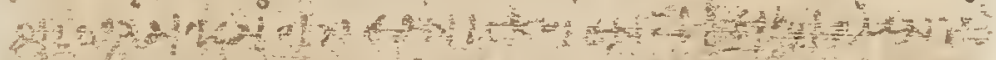

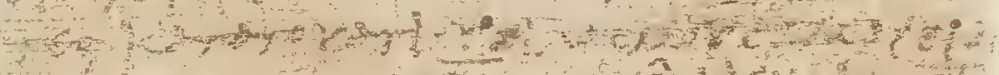

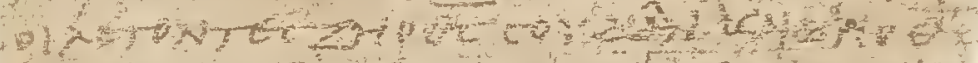

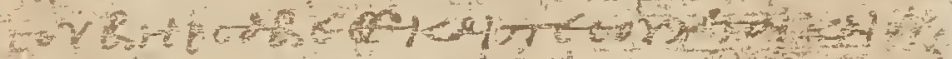

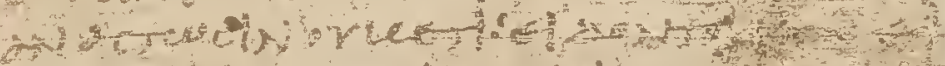

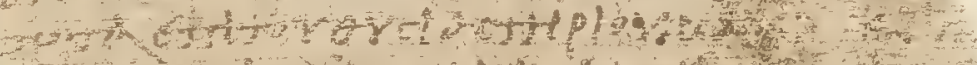

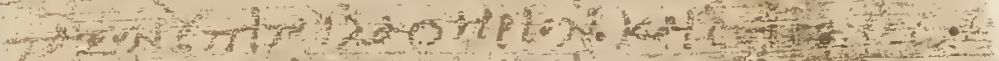
-

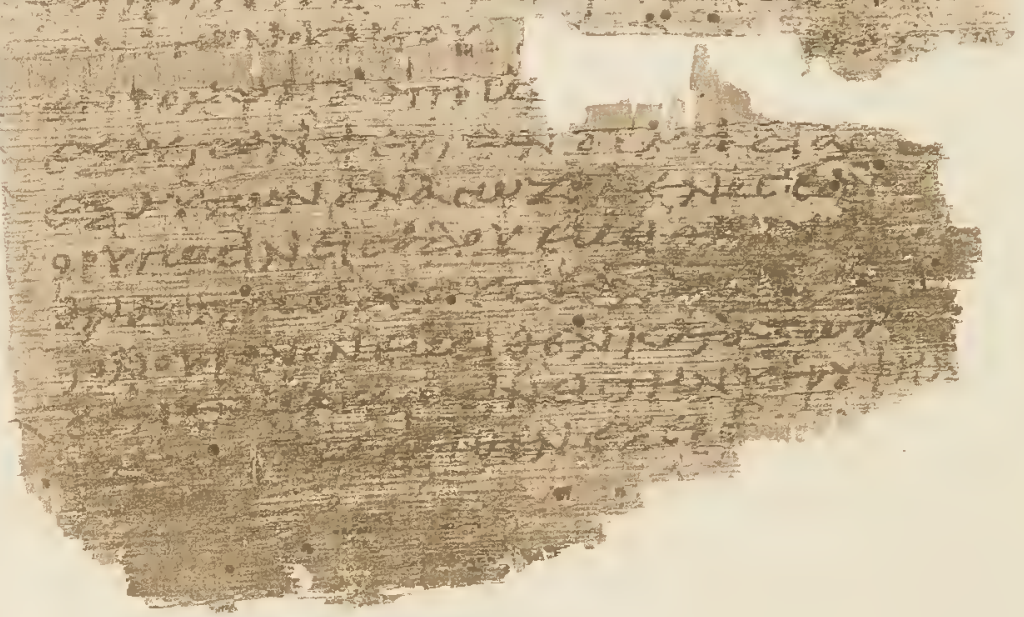

Amos viii, 4 -ix, 3. 


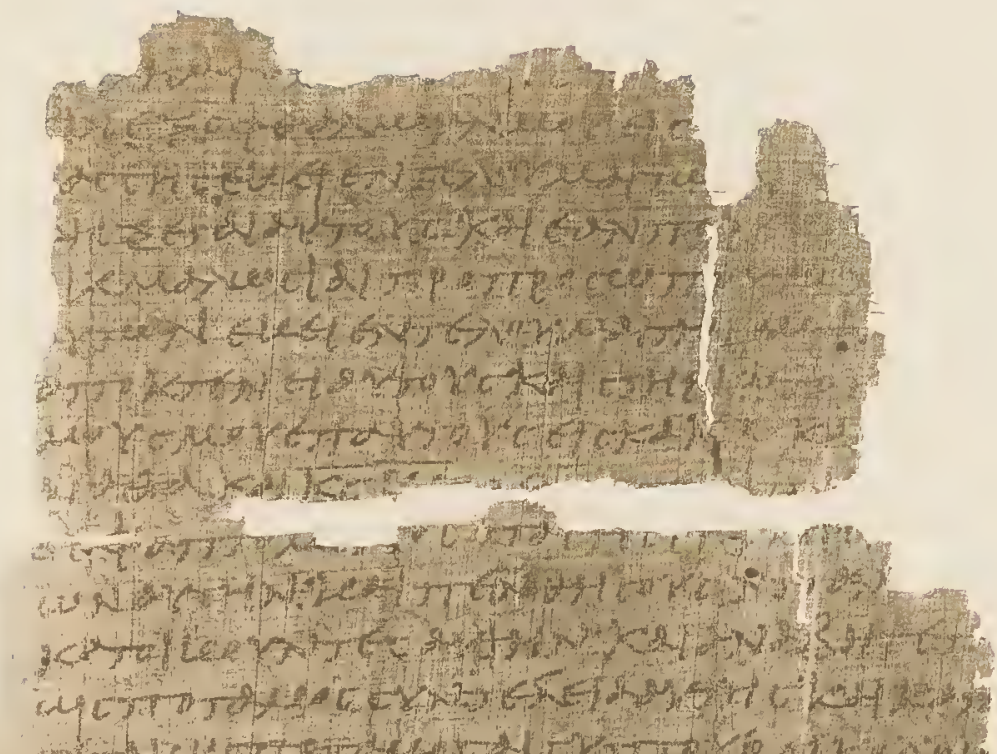

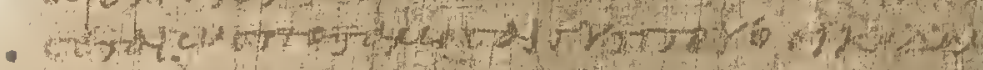

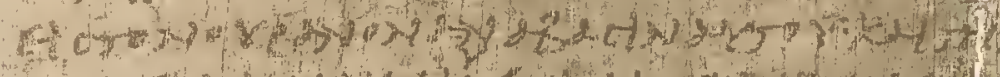
G. 6.,

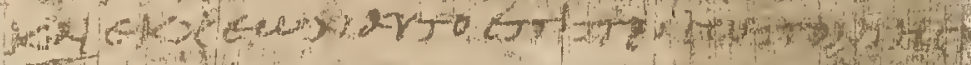
Fer J

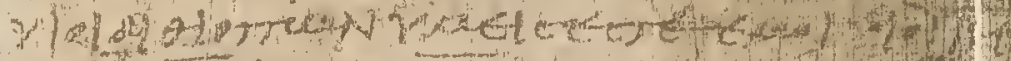

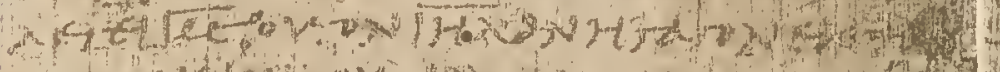

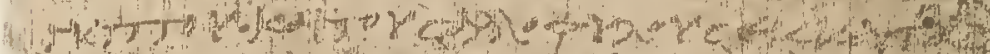

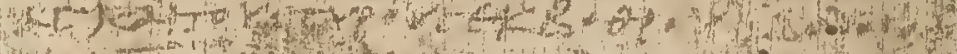
सelक

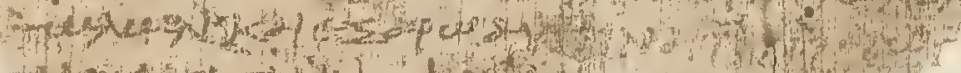

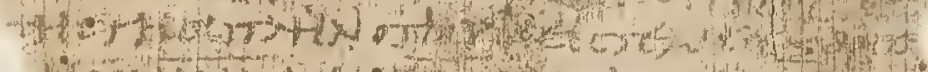

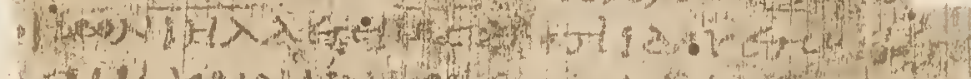

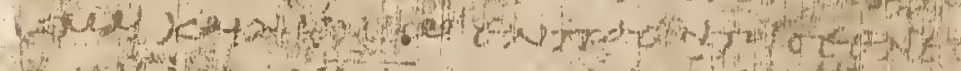
Tholn

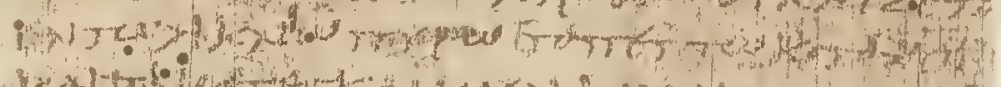

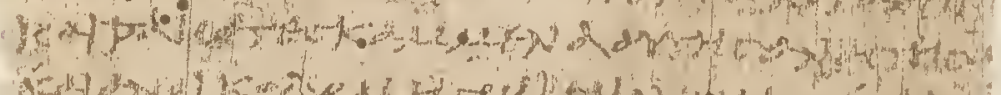
(10)

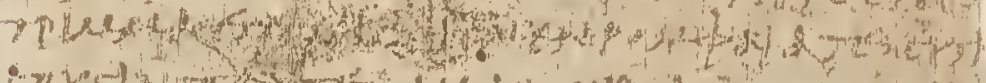
-vad

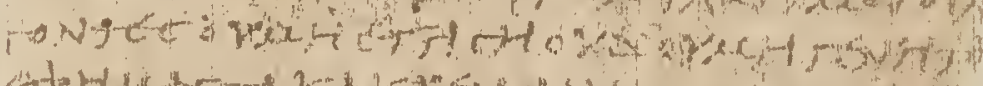

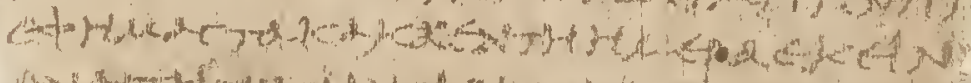
H W

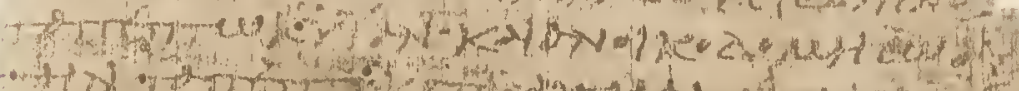

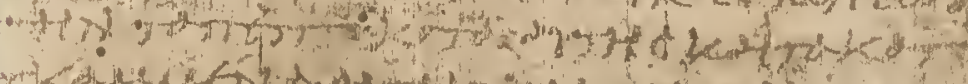

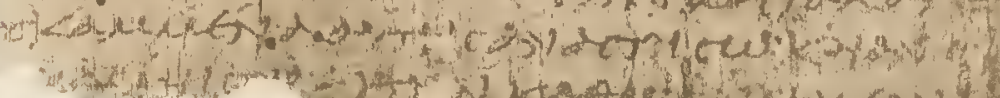

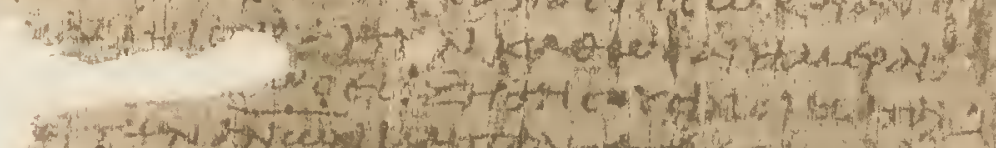

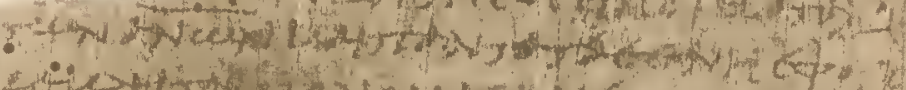

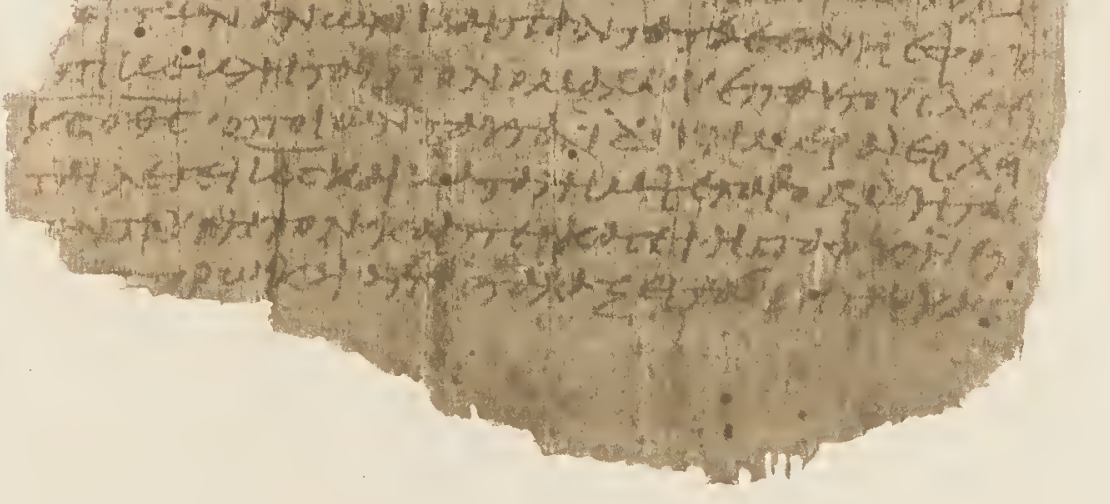




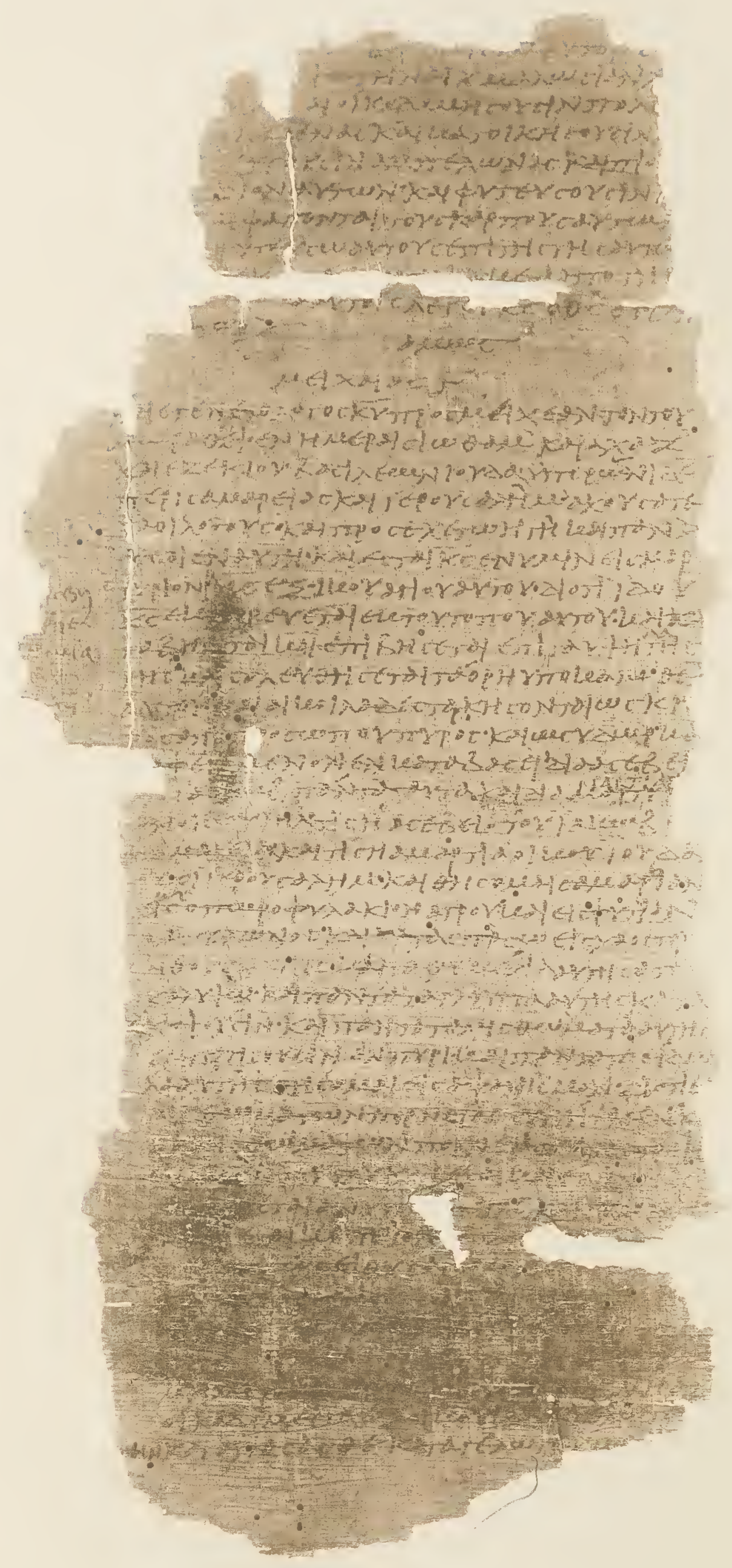

Inos ix. 13-Micias i, 11. 


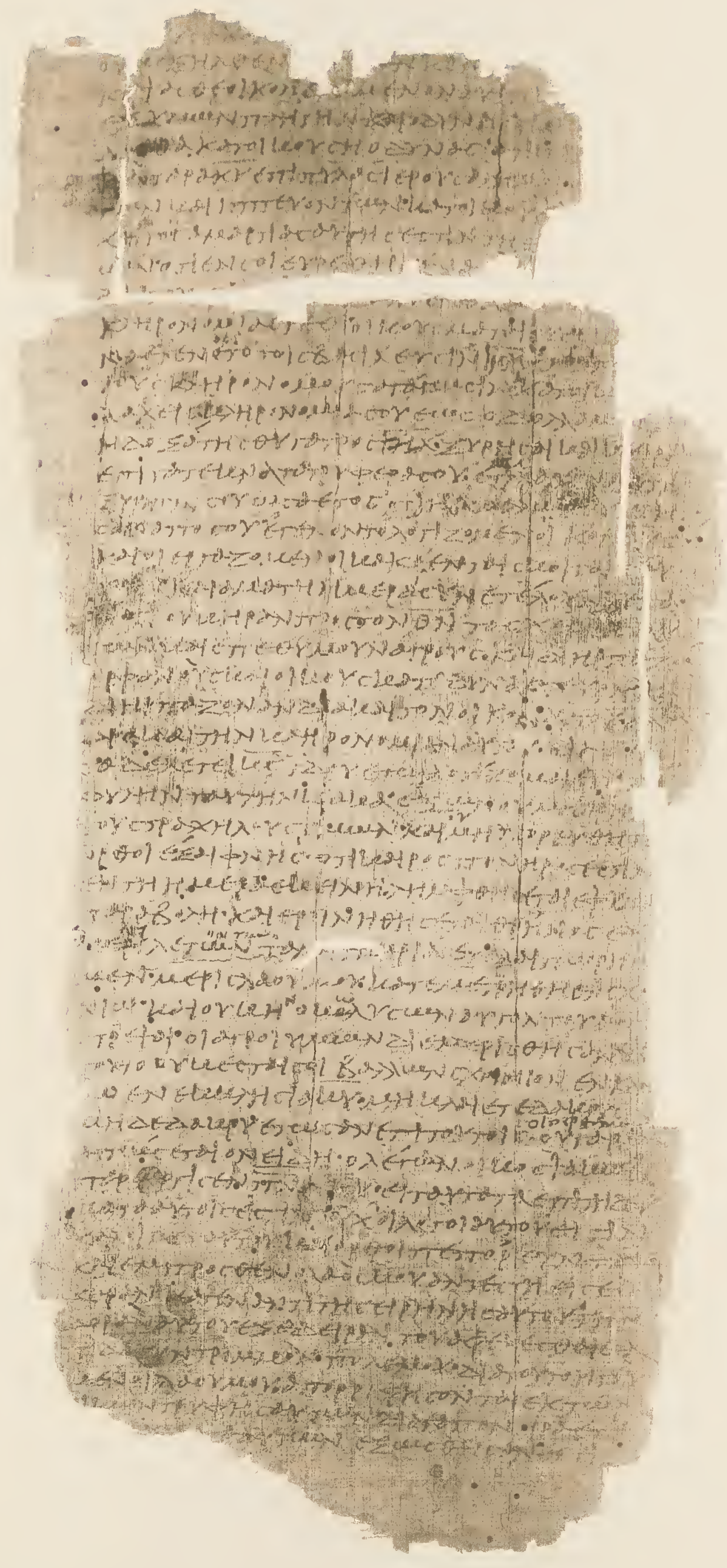

Michas i, 11 - ii, 9. 


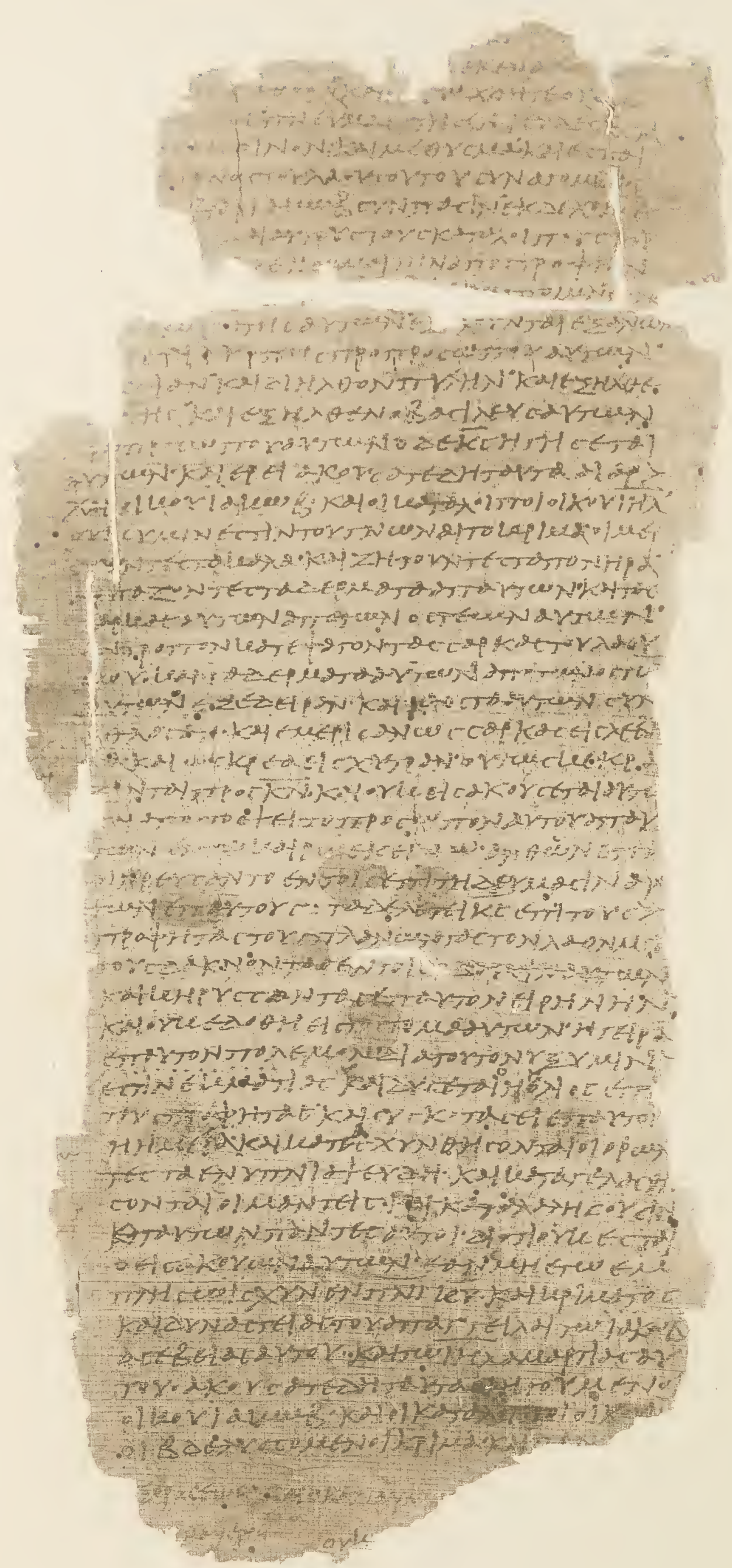

Michas ii, 9 - iii, 9. 


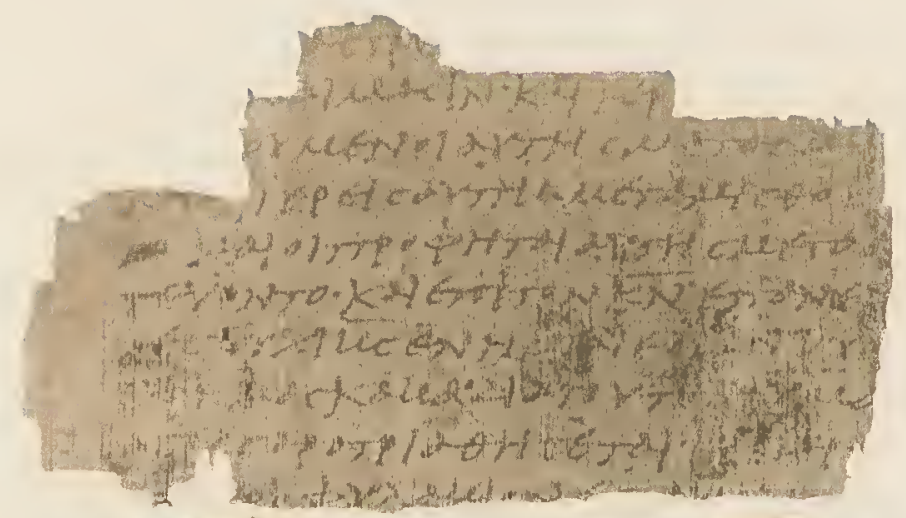

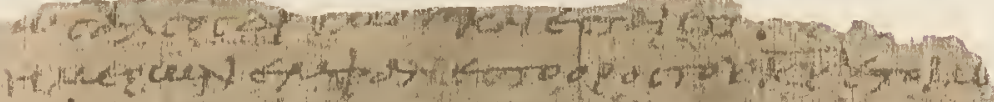

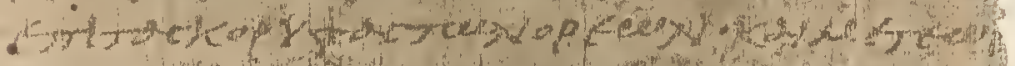

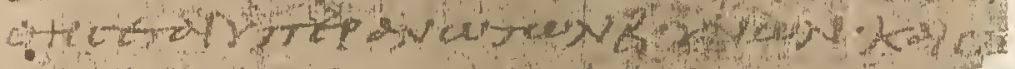

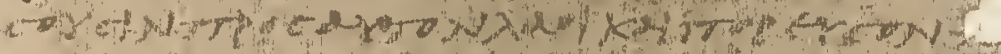

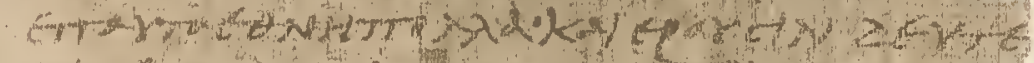

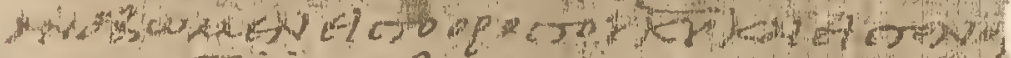

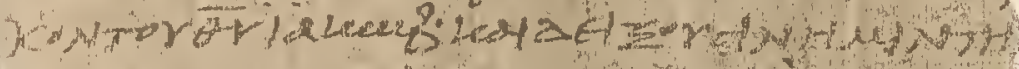
THN

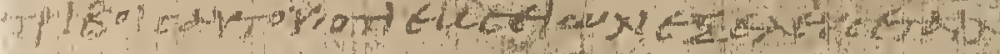

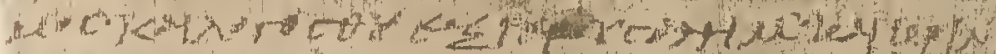

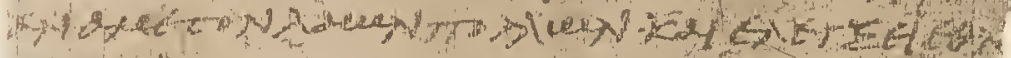

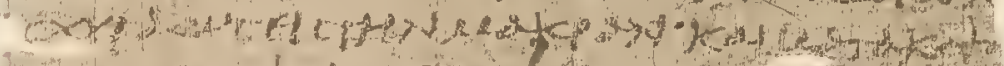

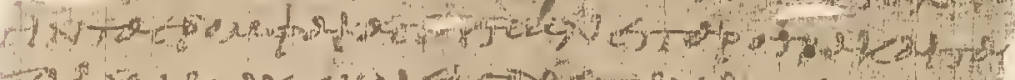

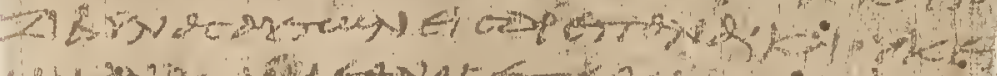

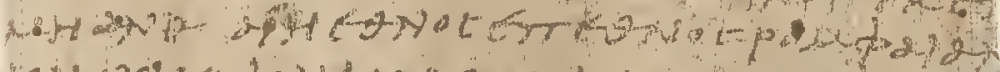

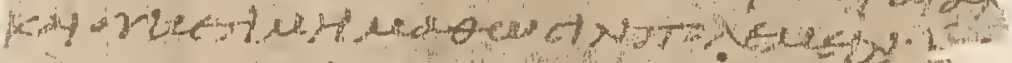

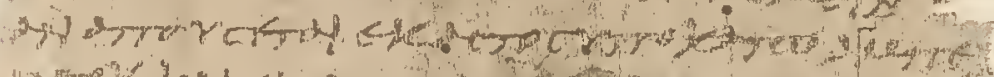

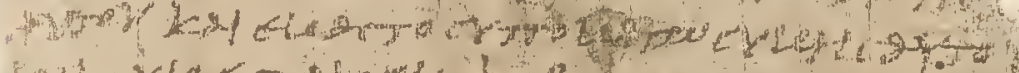

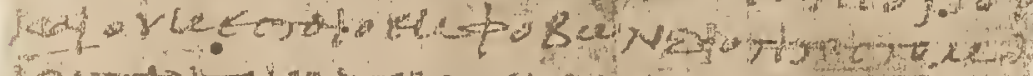

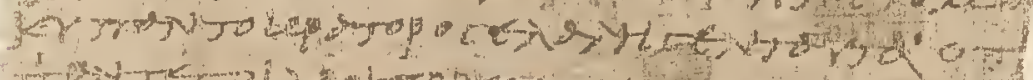
त)

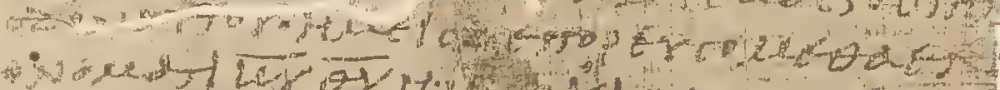

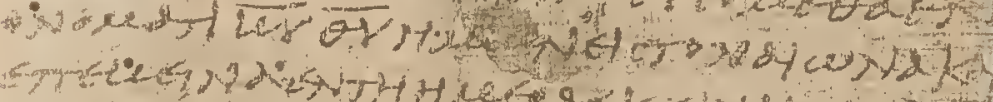

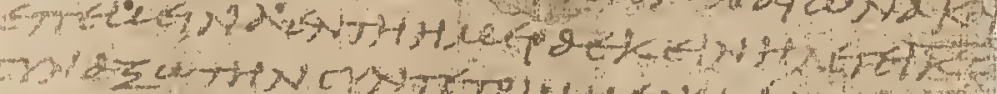
-.. दूस -

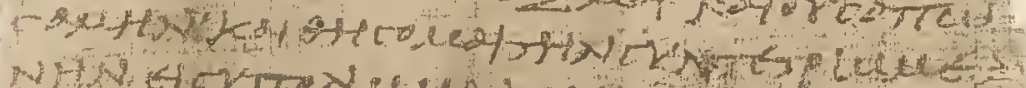

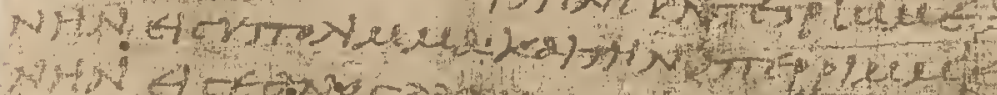

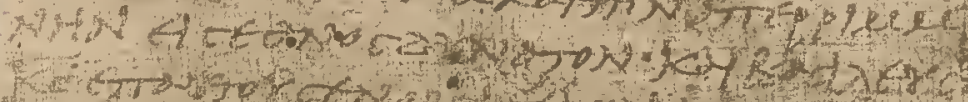

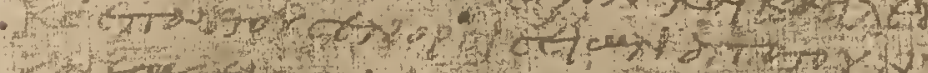

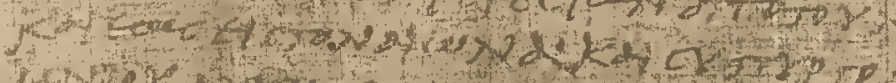

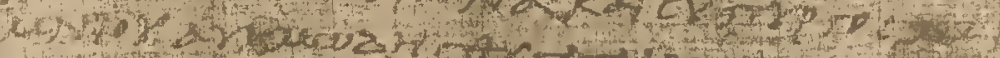

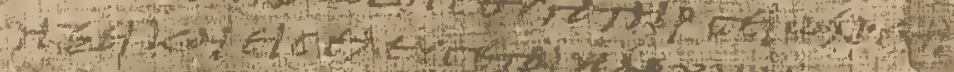

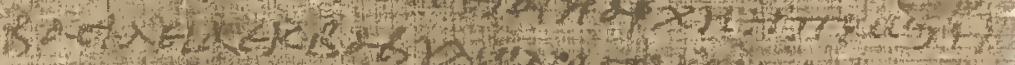

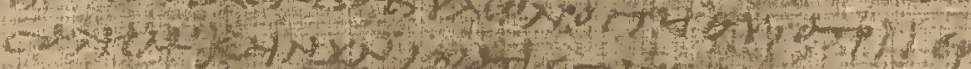

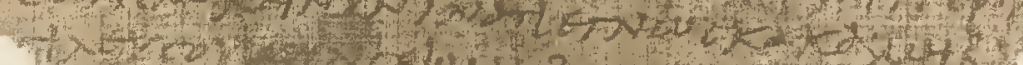

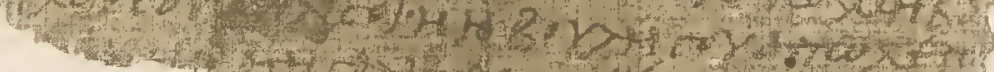

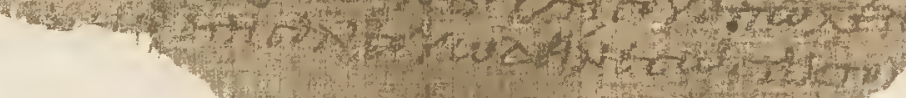

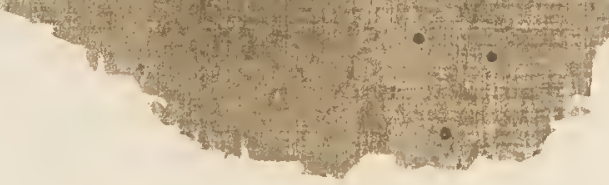




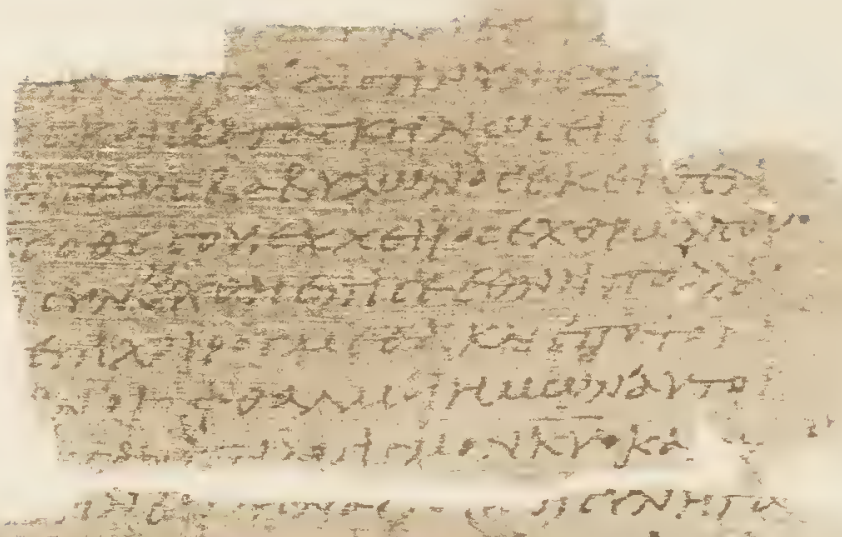

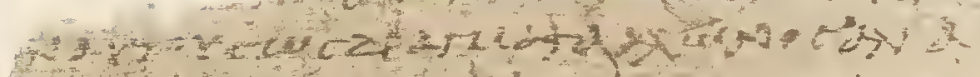

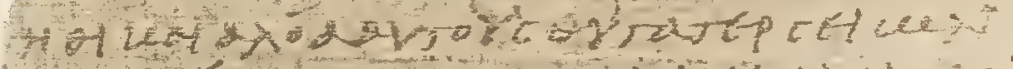

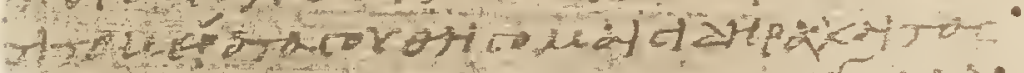

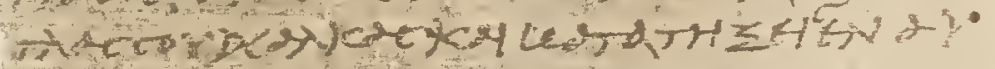

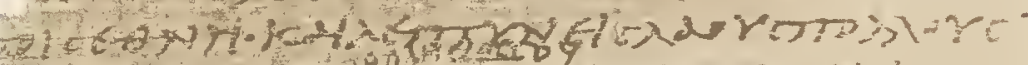

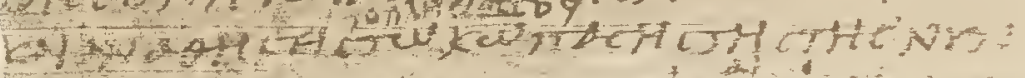

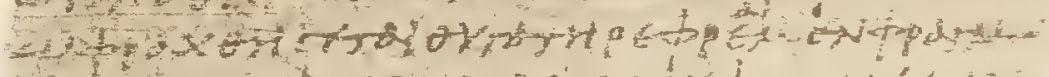

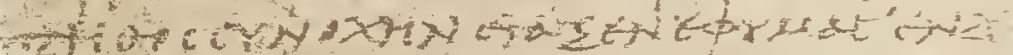

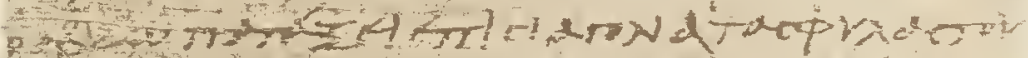

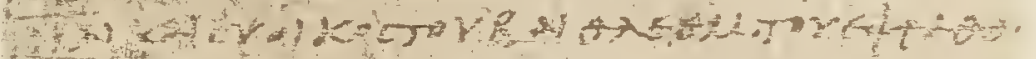

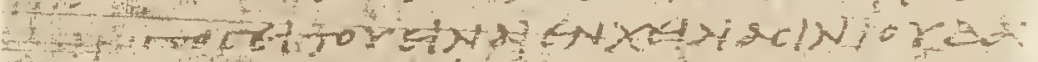

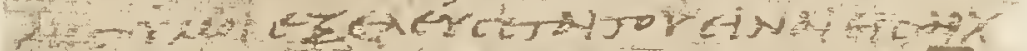

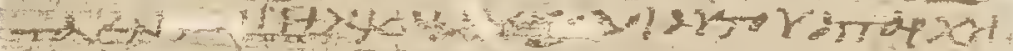

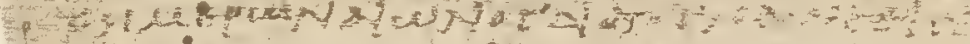

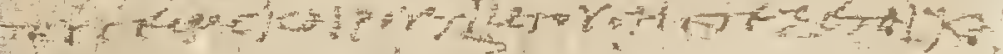

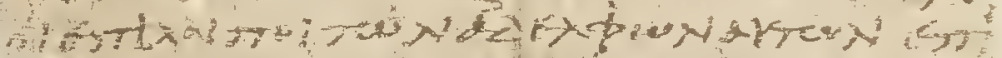

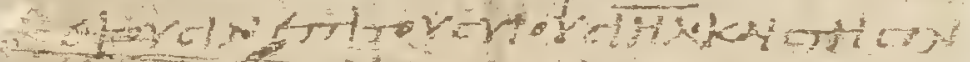

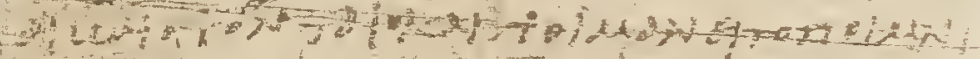
MH

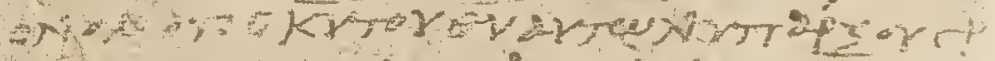

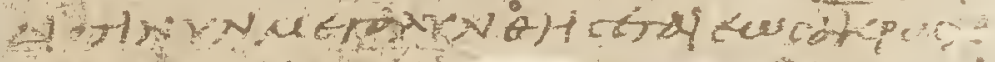

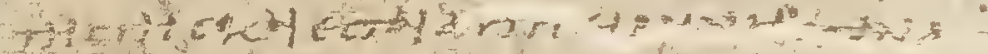
-

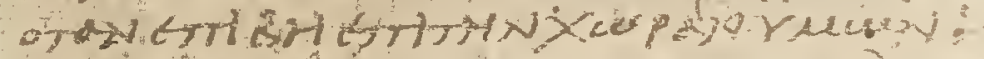

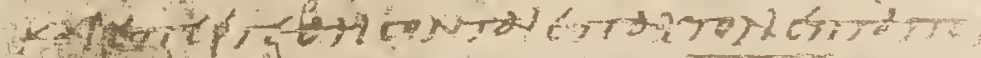

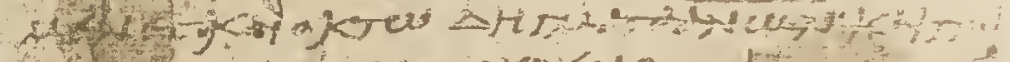

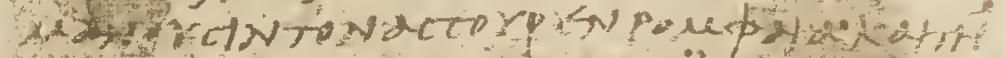
Mnf

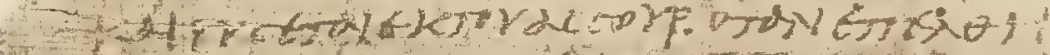

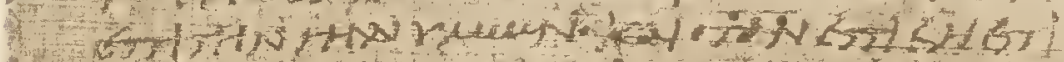

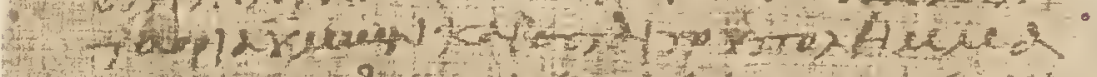

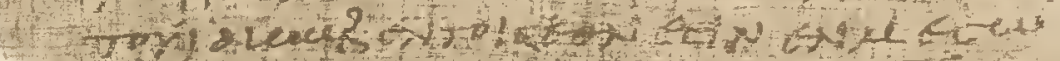

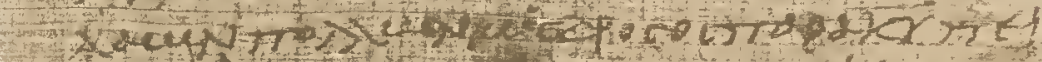

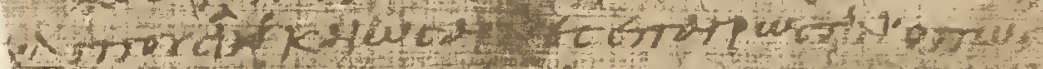

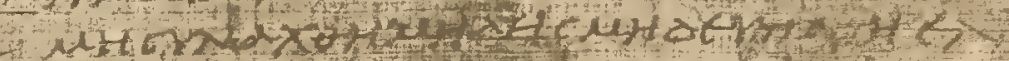

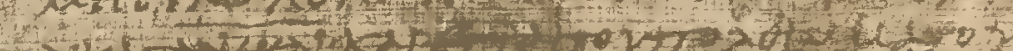

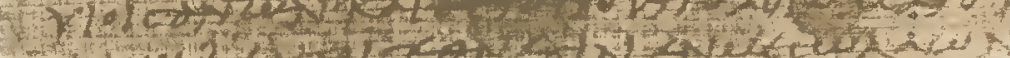

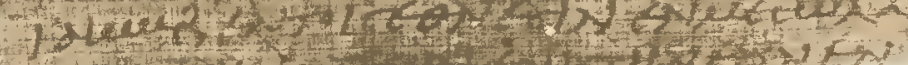

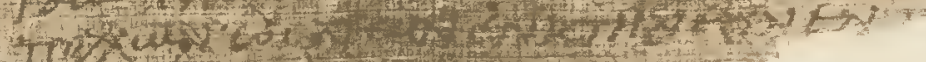

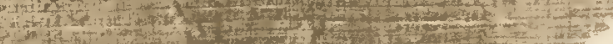

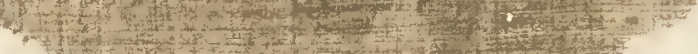

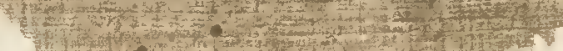

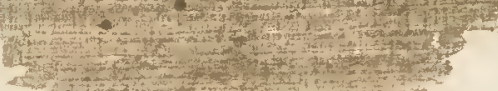

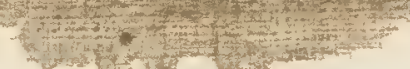




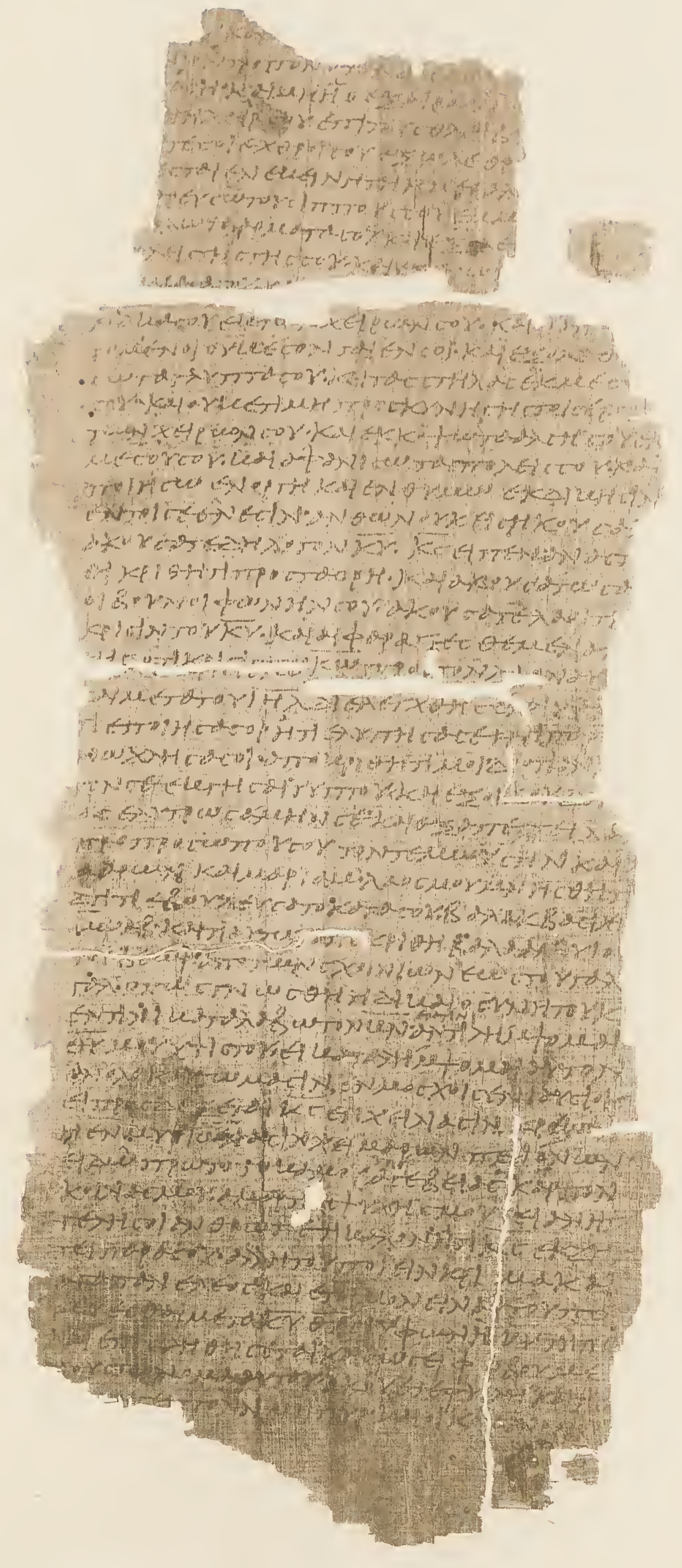

Mlemas $\mathrm{r}, 8-v i, 10$. 


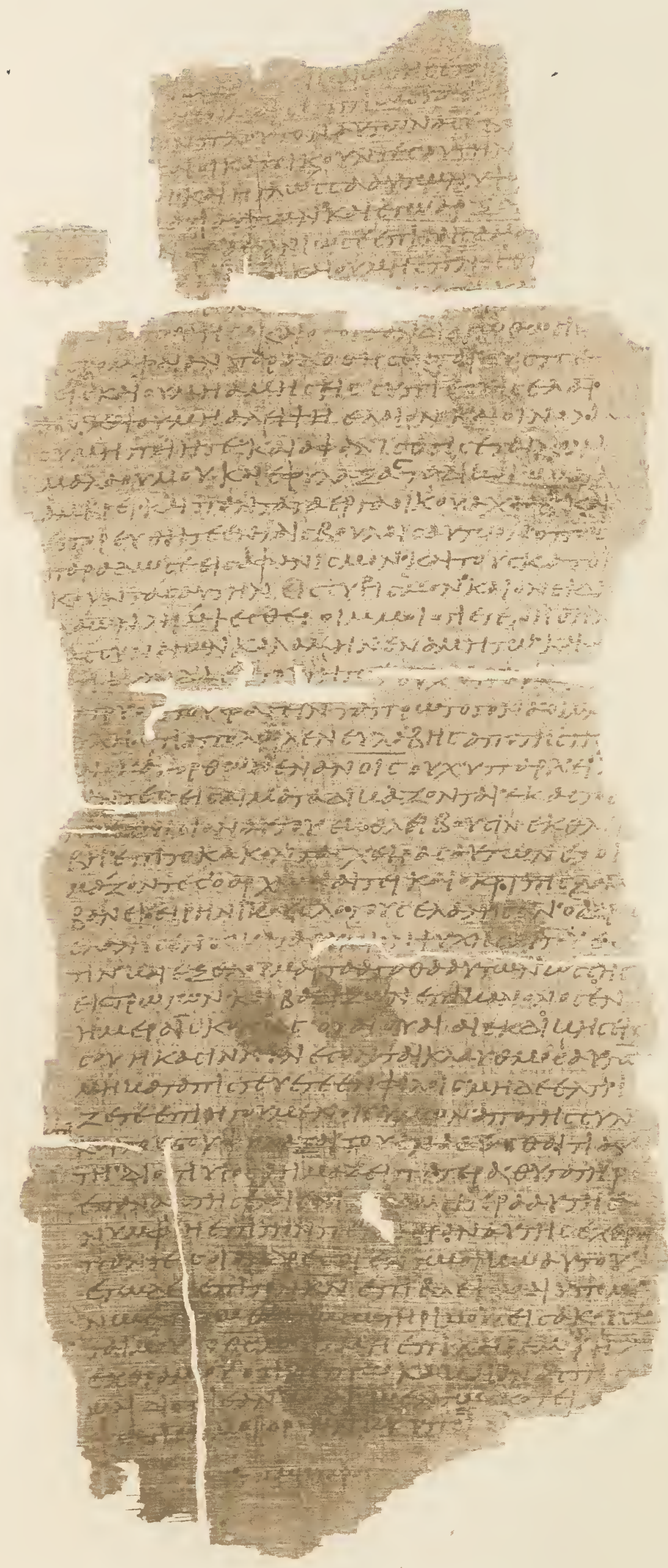

Jichas vi, 10-vii: 9. 


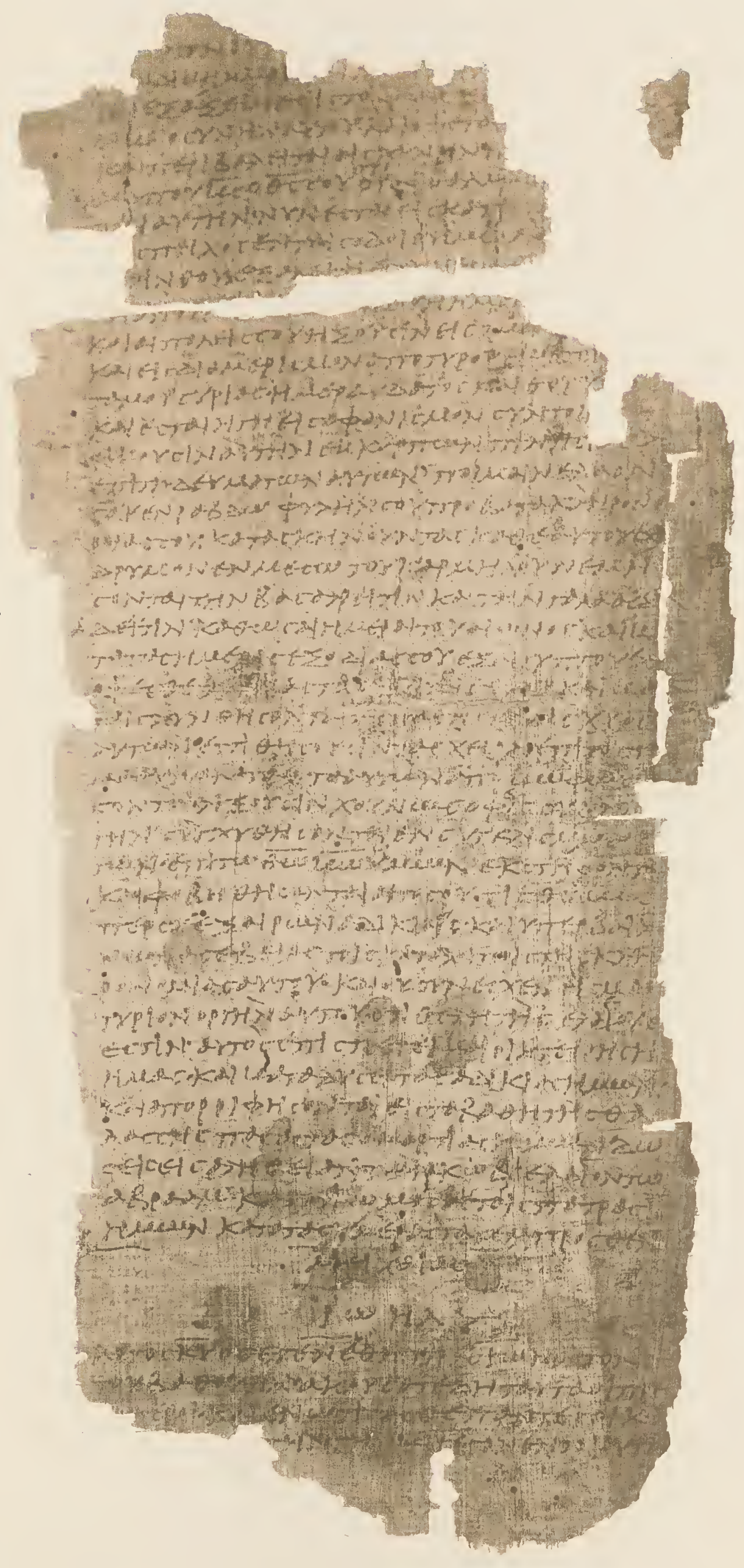




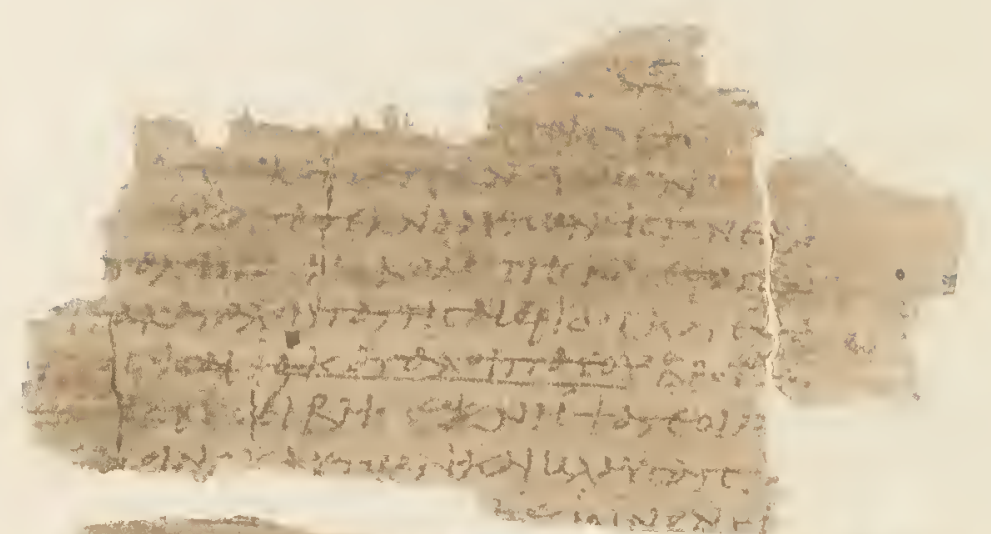

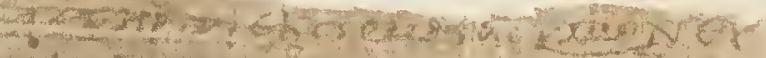
Nhx

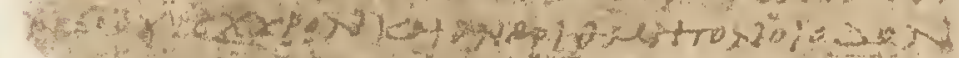

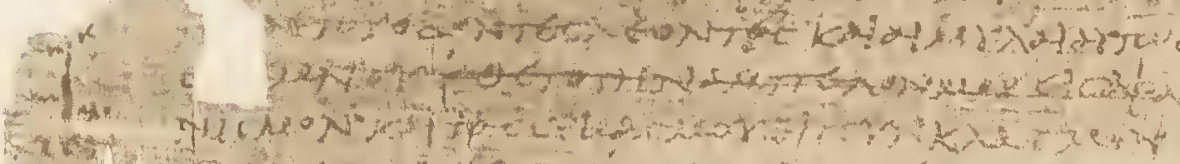

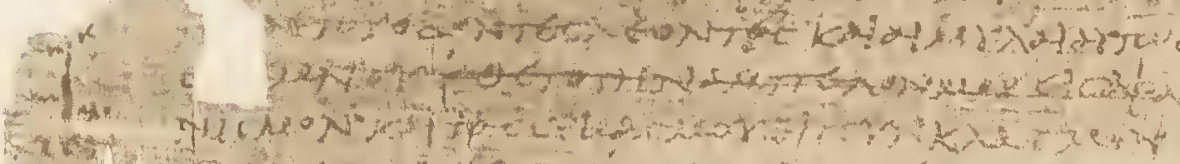

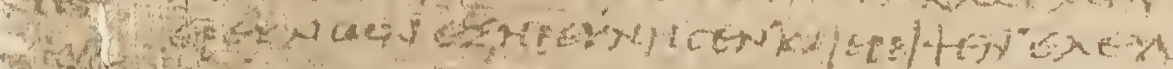

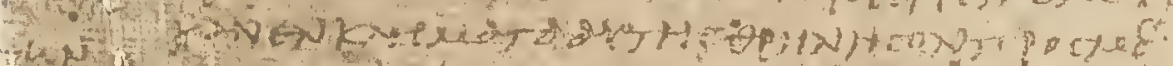

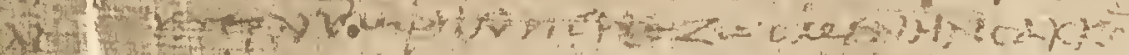
X. Whe bor

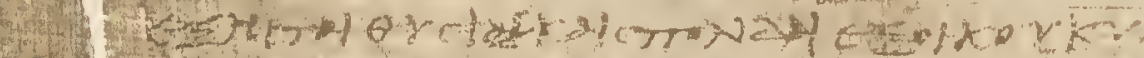

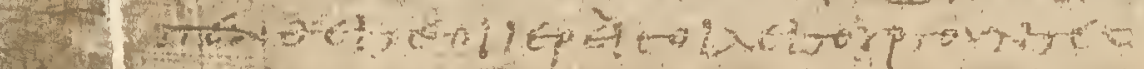

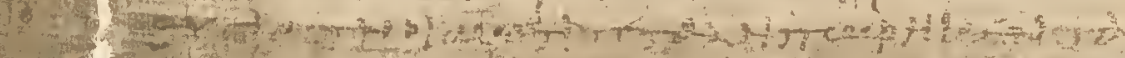

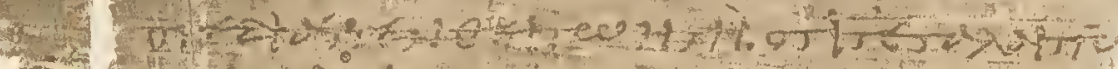

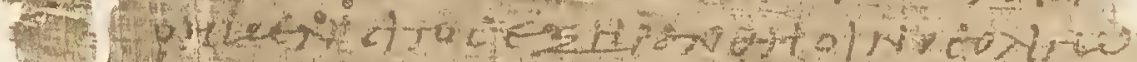

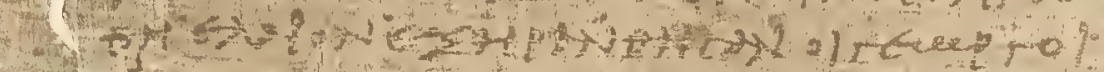

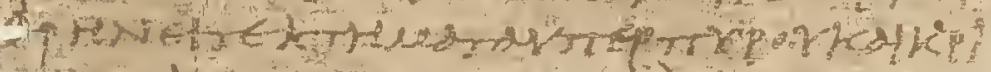

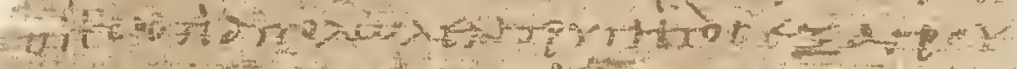

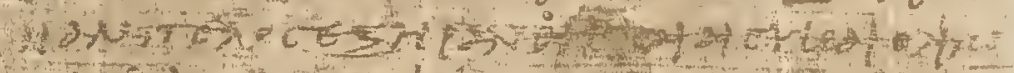

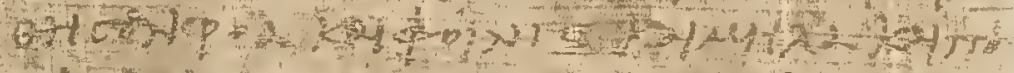

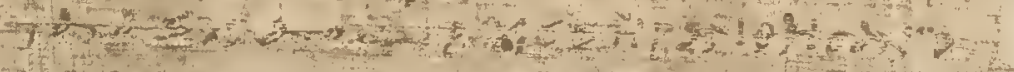

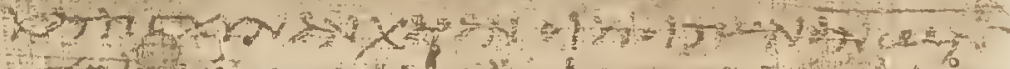

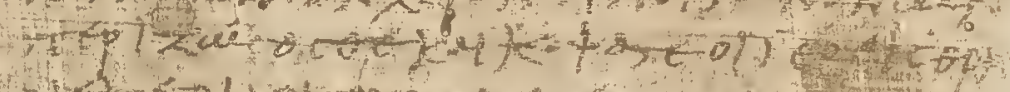
Jet $A c-00$ H

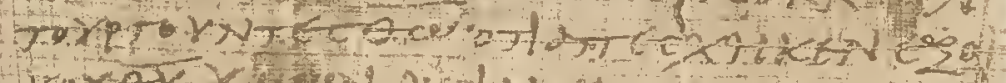

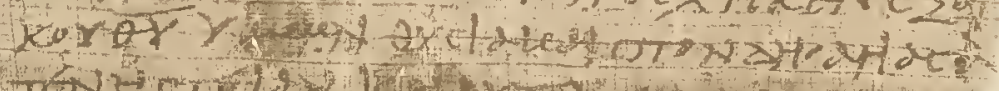

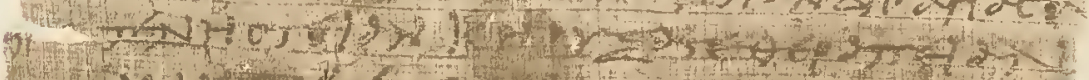

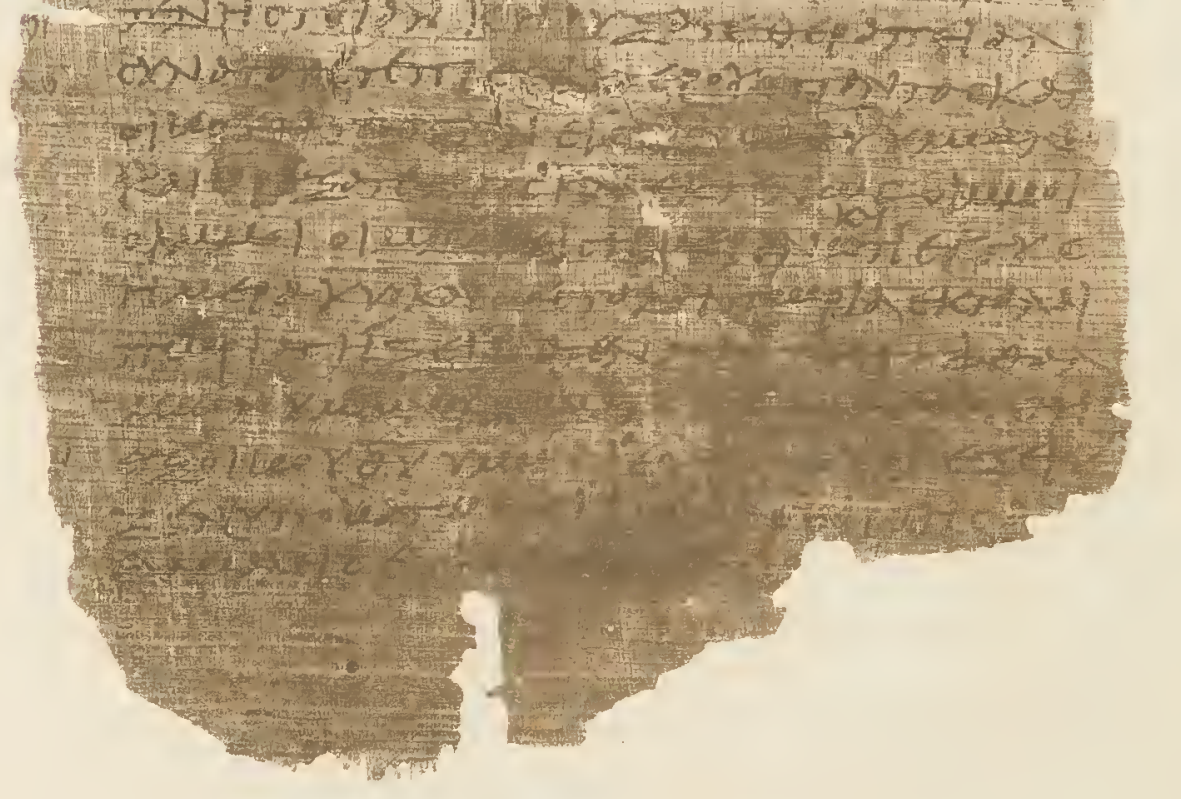

JoEL $\mathrm{i}, 2-17$ 

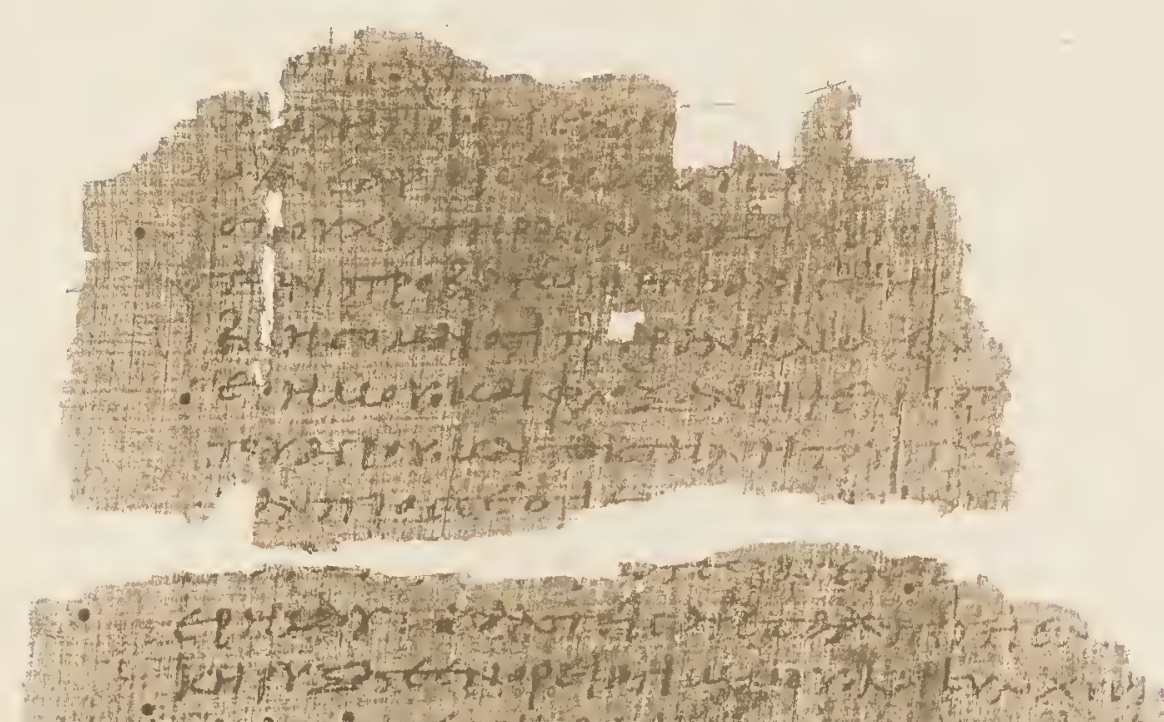
- f

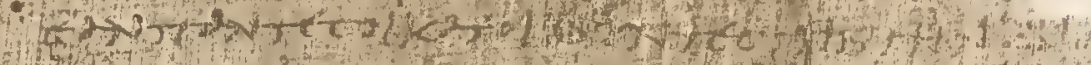

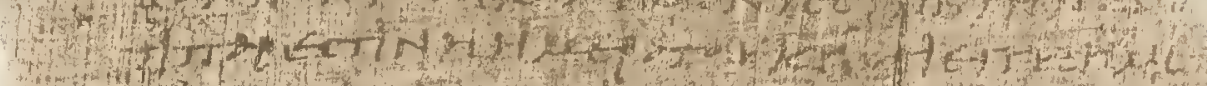

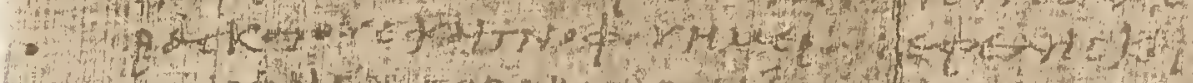

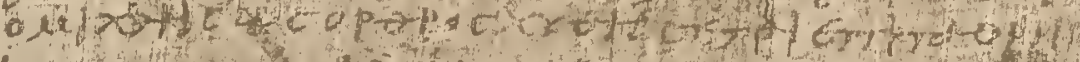

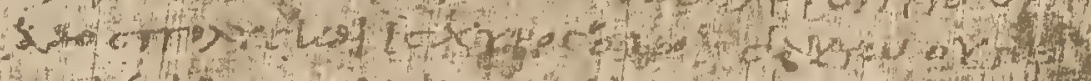

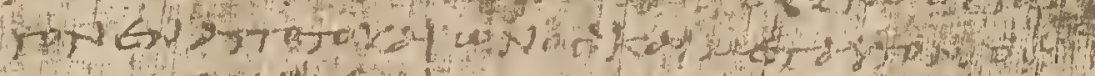

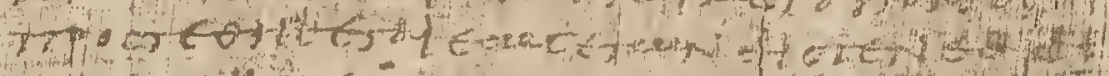

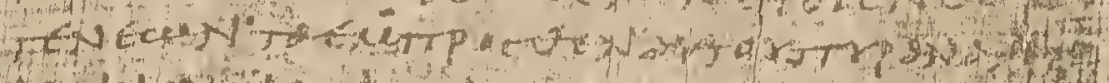

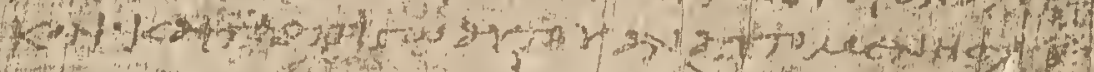

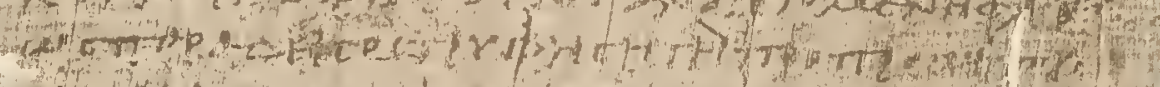

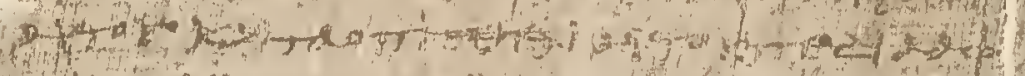

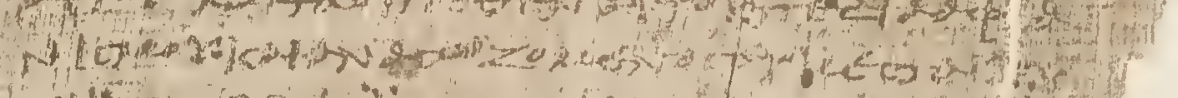

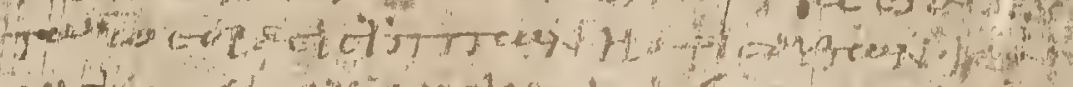

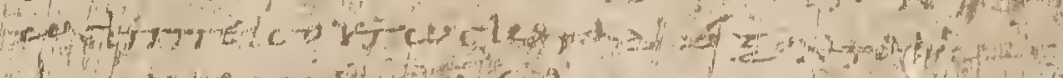

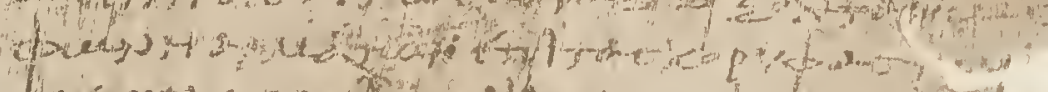

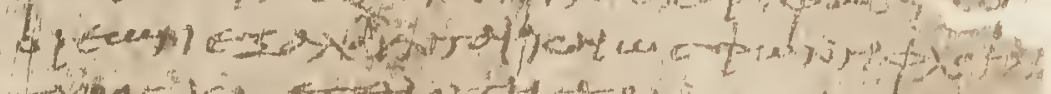

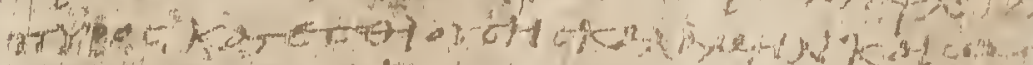

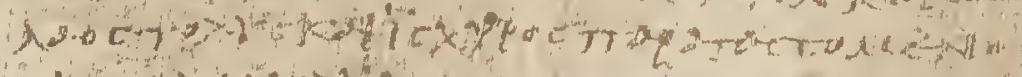

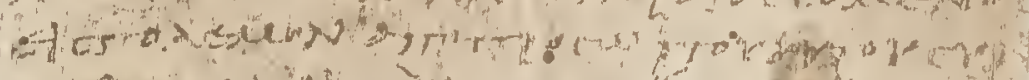

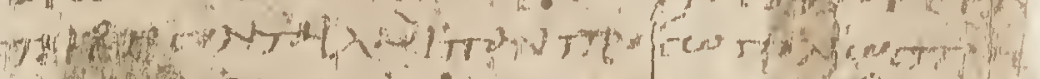

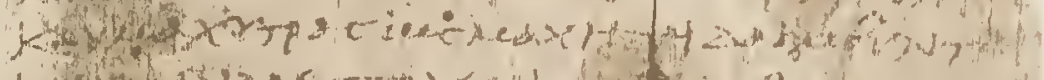

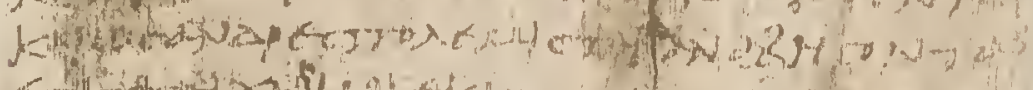
Koth

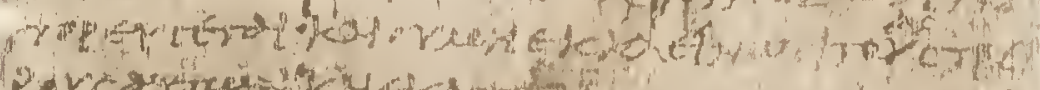

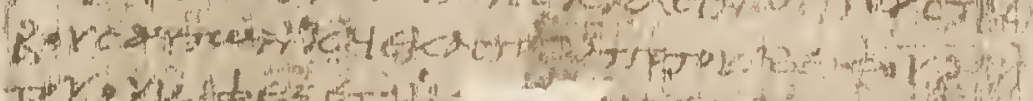

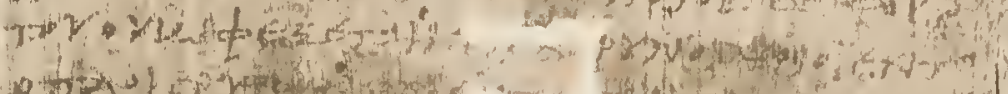

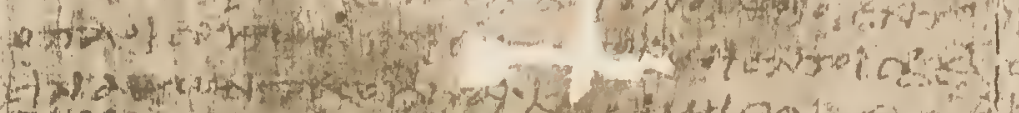

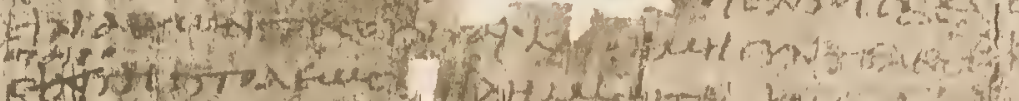

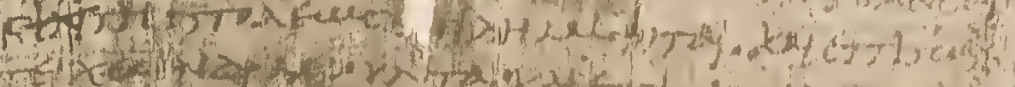

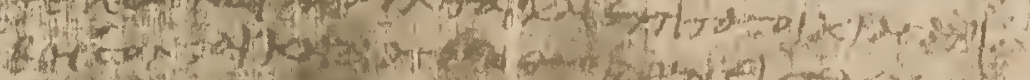

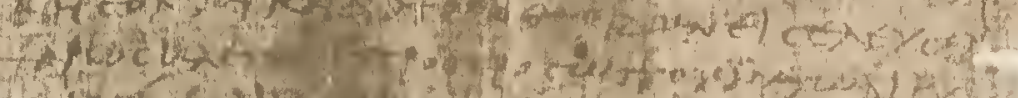

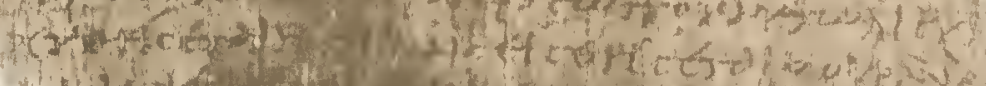

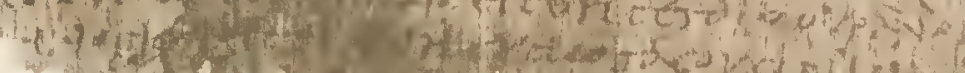
4.

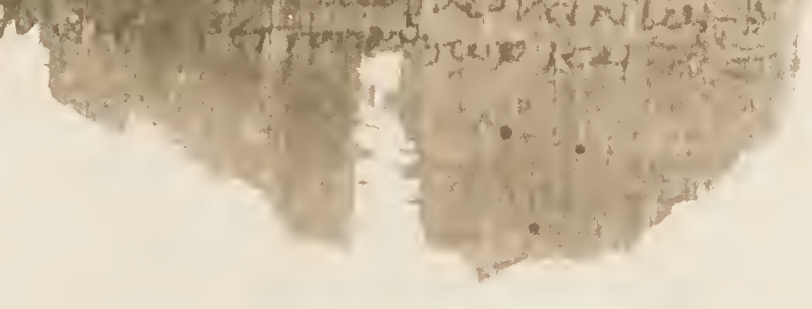




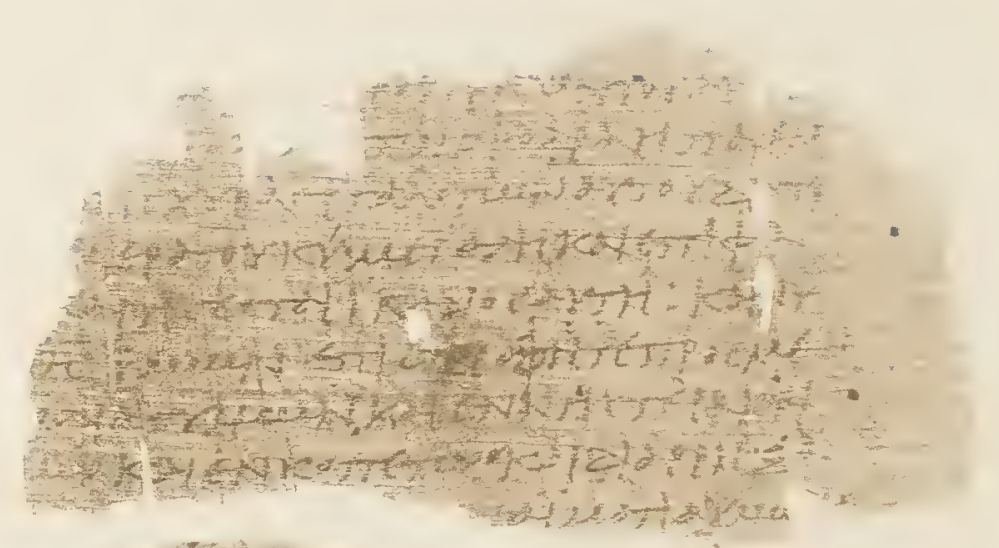

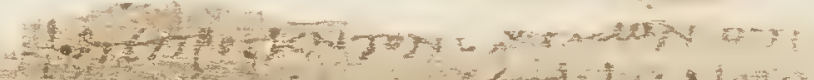

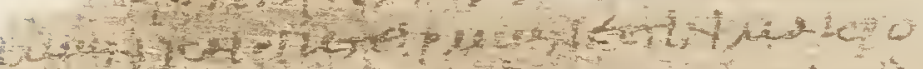

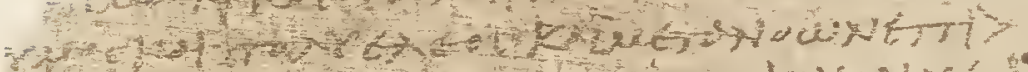

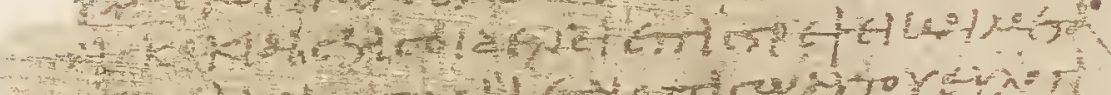

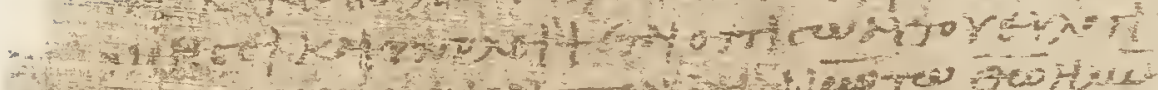

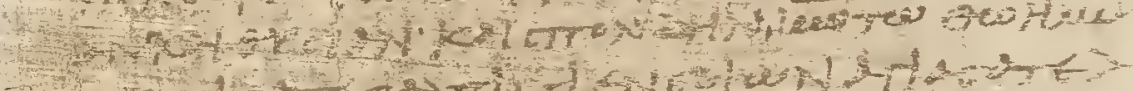

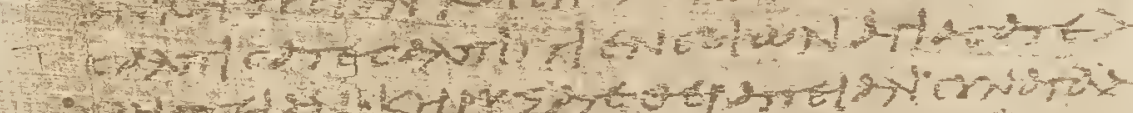

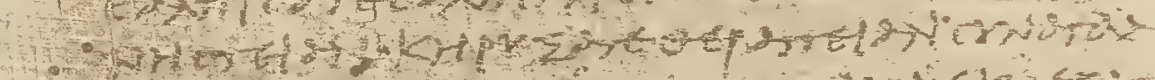

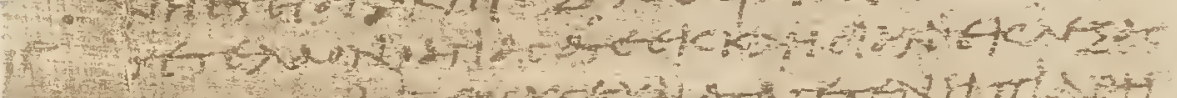

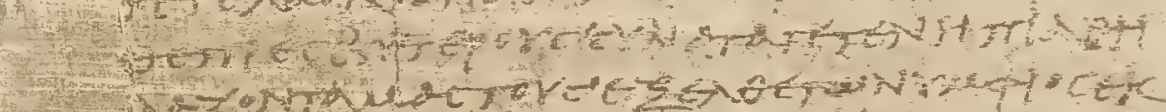

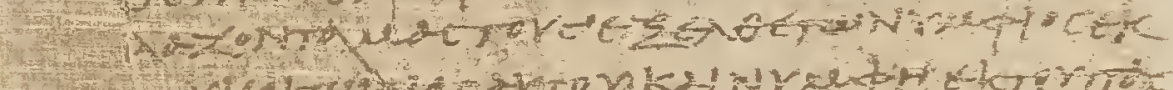

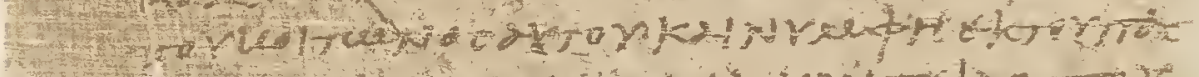

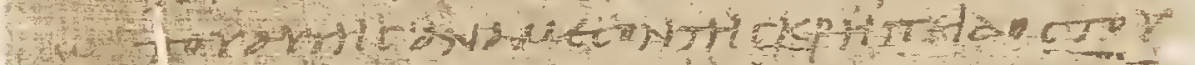

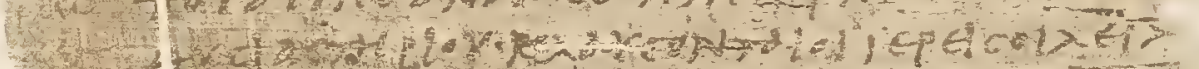

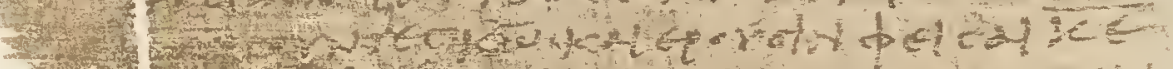

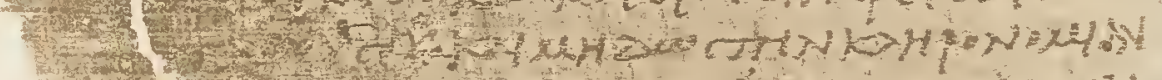

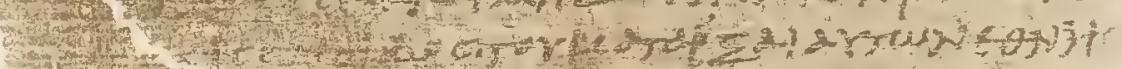

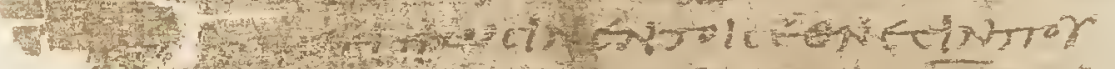

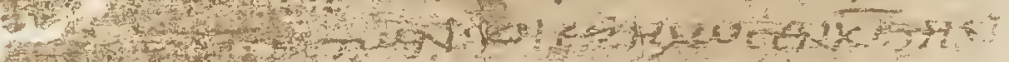

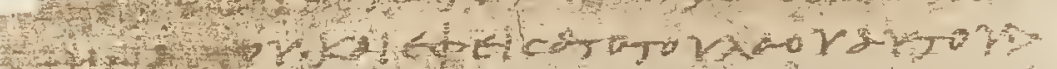

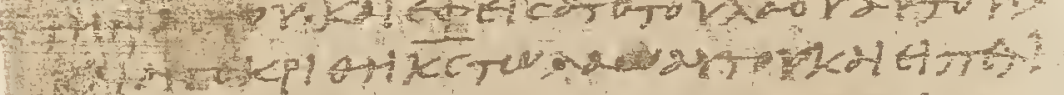

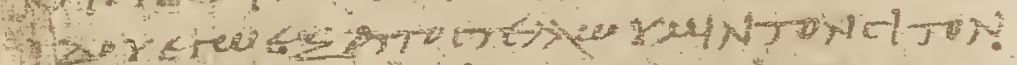

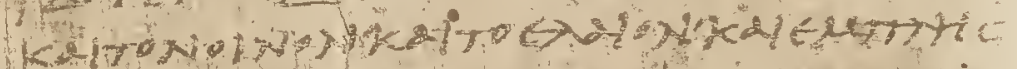

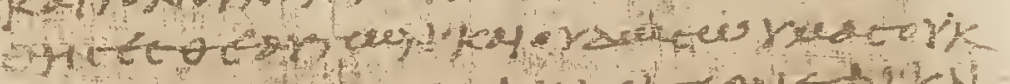

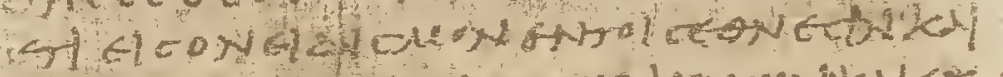

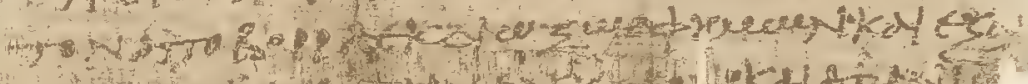

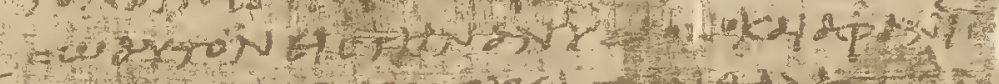

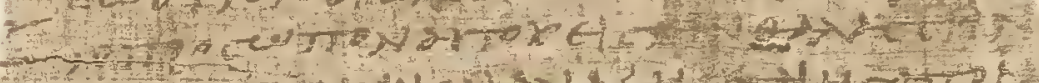

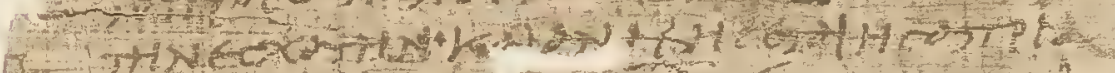

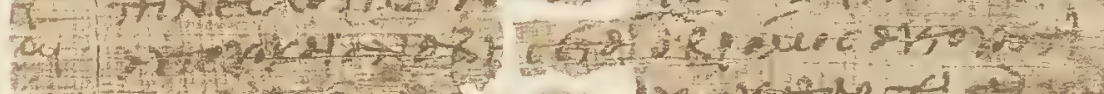

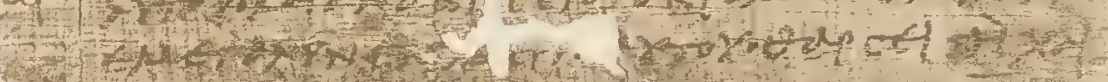

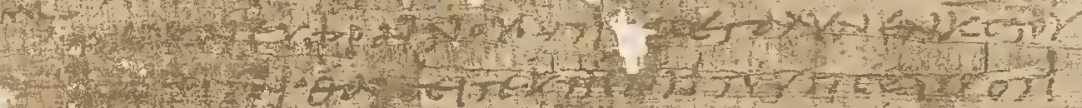

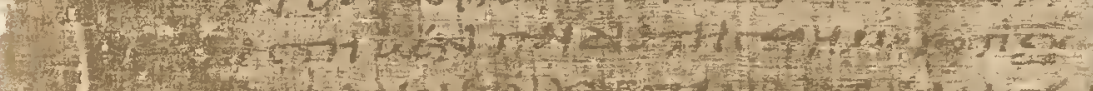

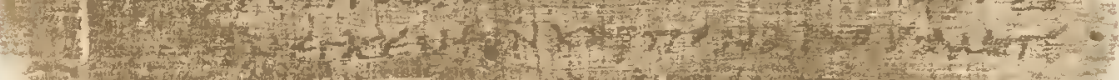

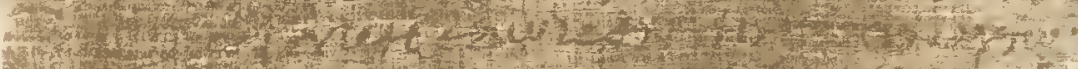

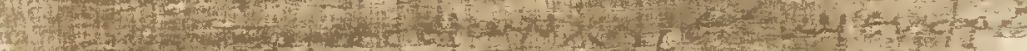

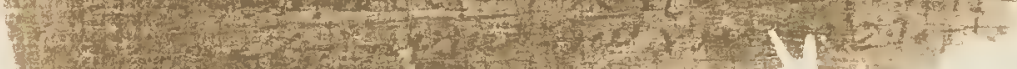
(2)

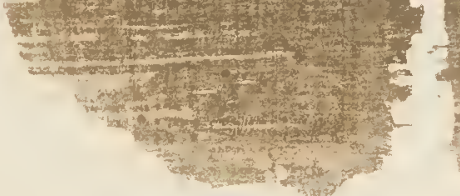

JoEL ii, $11-23$. 

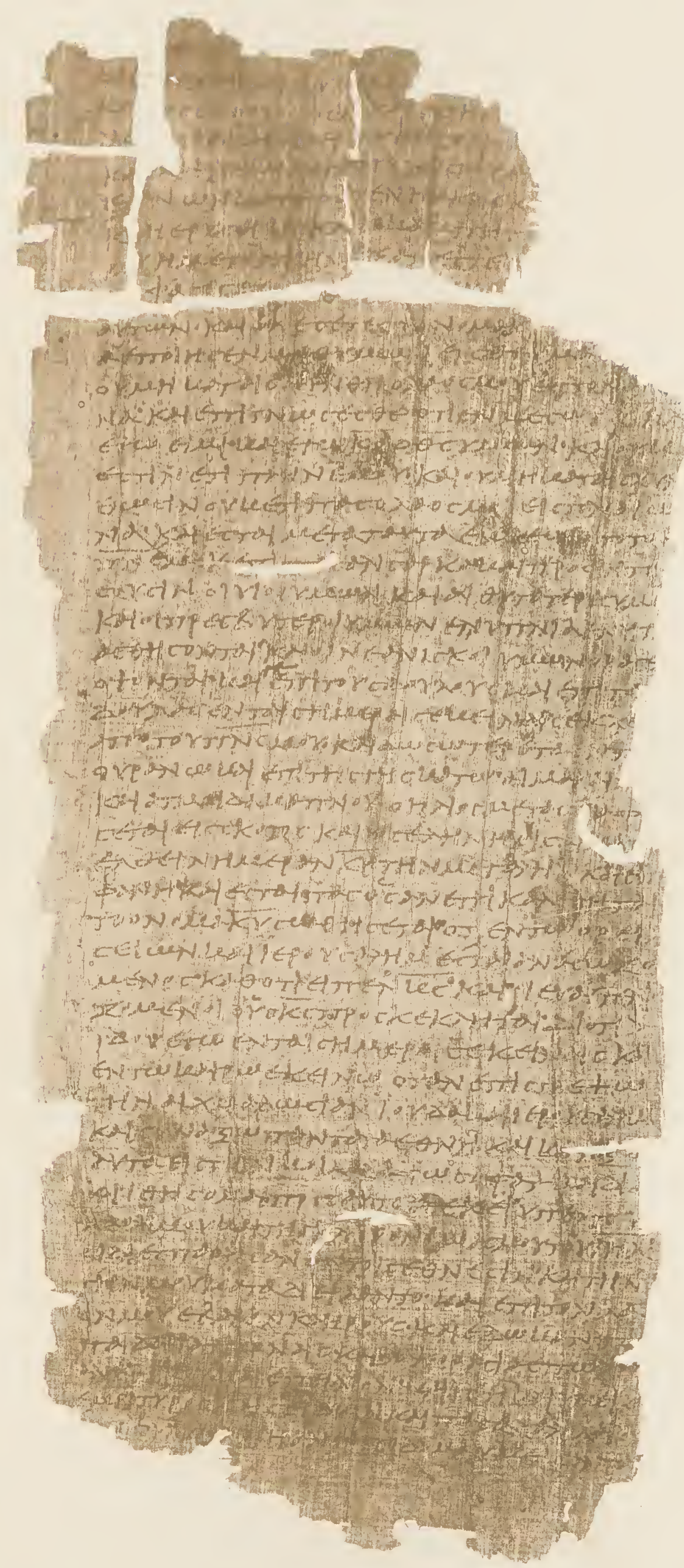

Jofe ii: $23-$ iii: 4. 


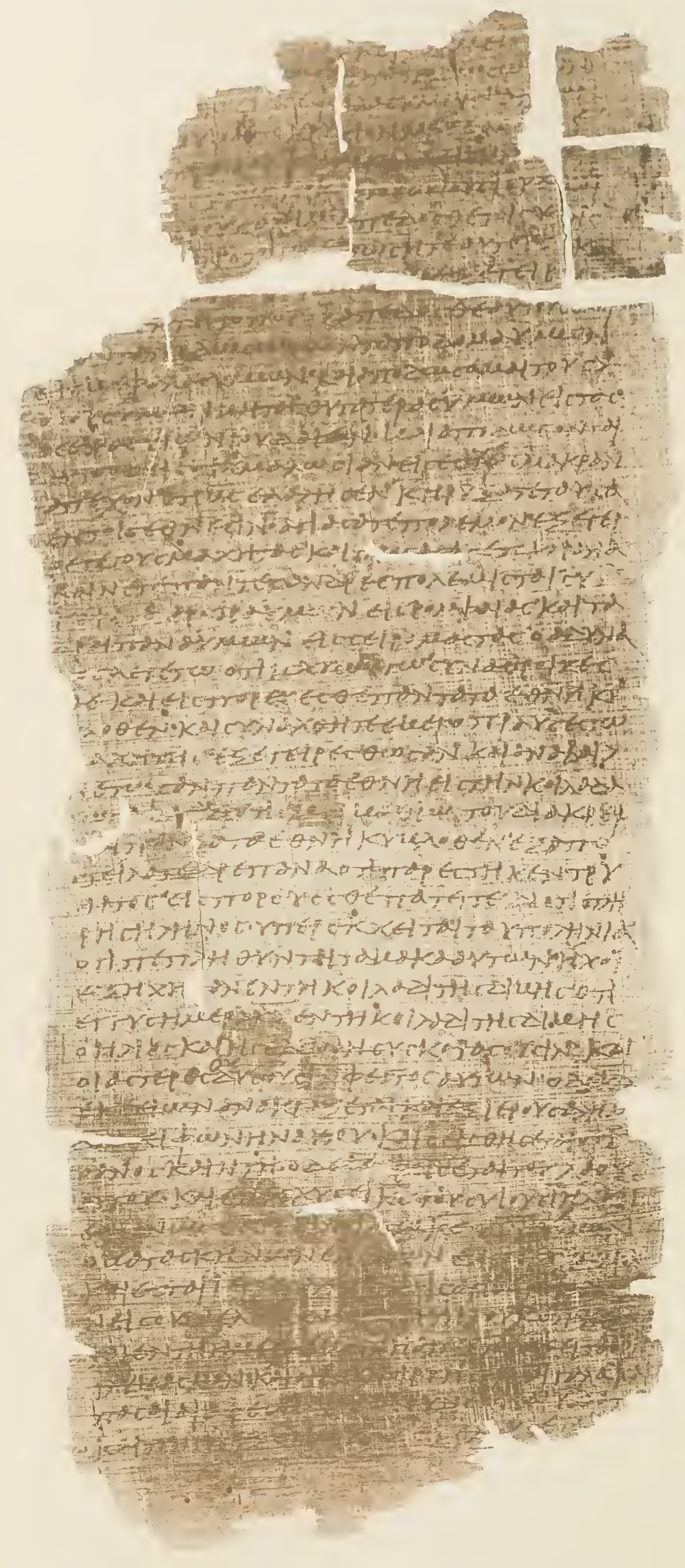



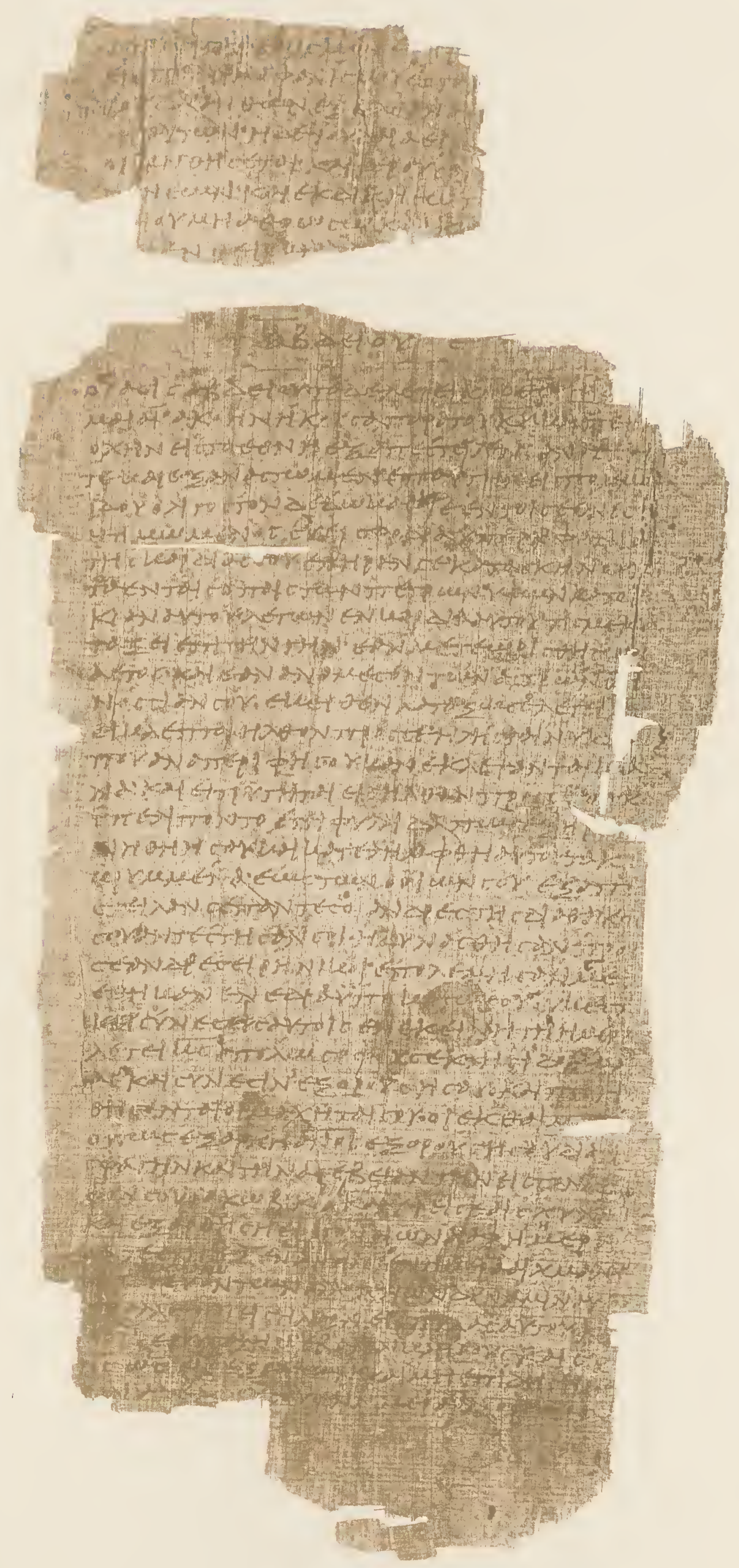

Joli, iii, 18-Obanias 12. 


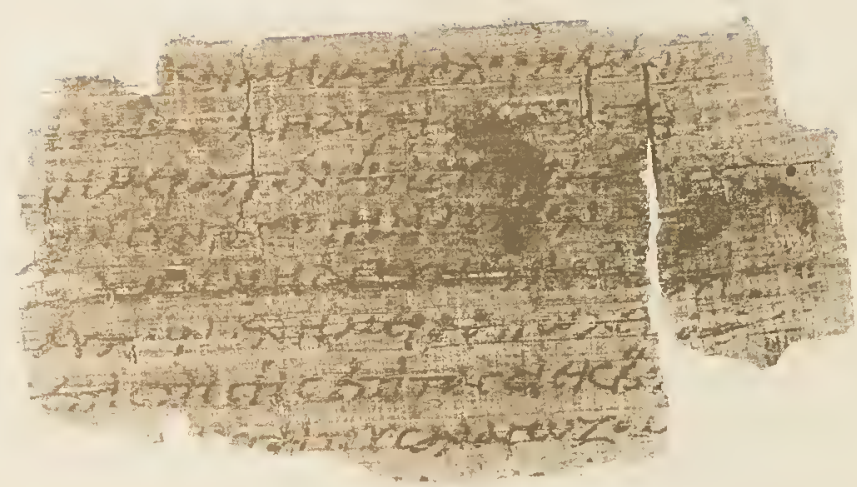

XETTE

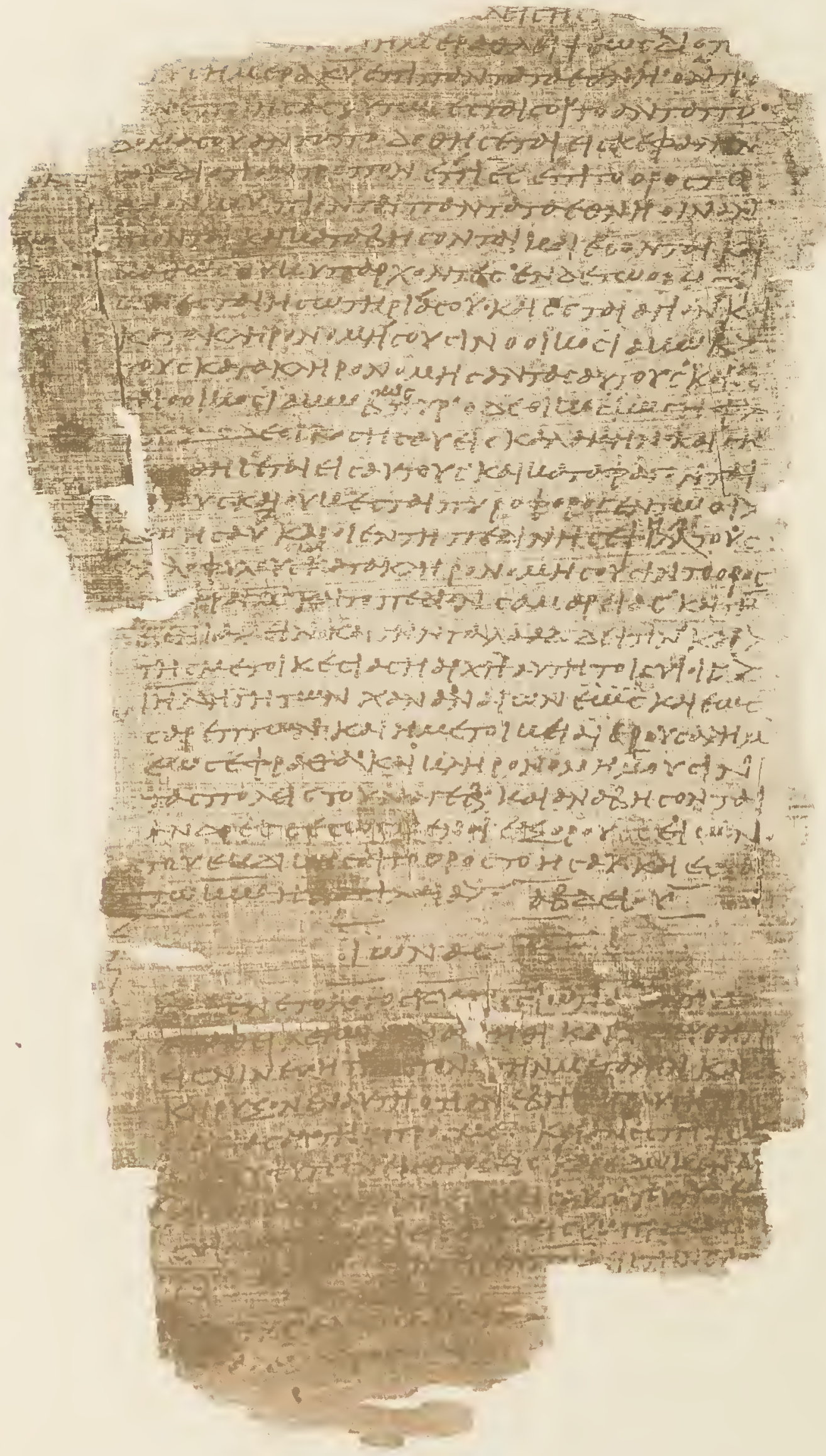

Obadias 12 Jonas $i, 4$. 


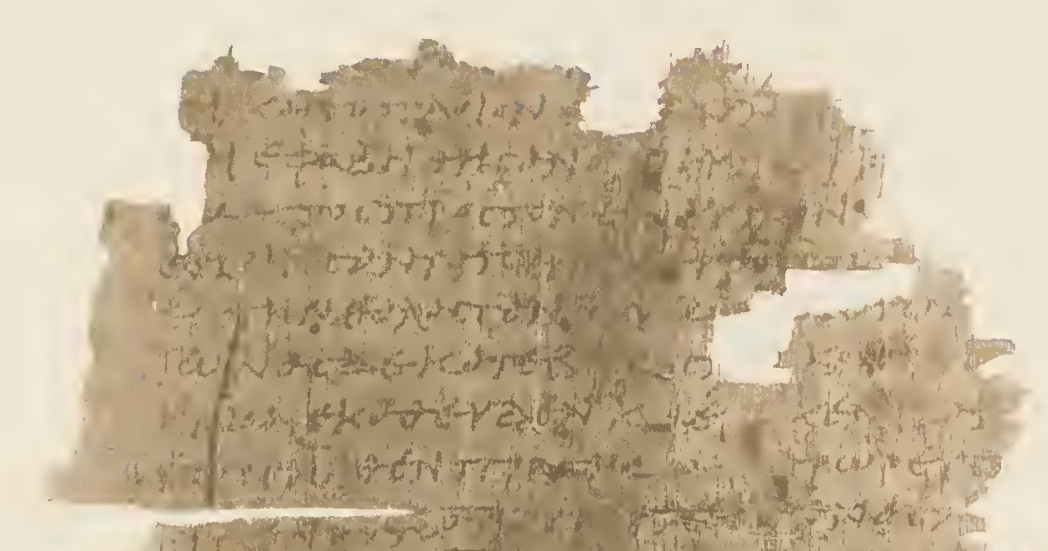

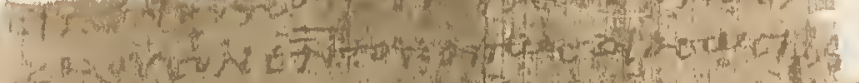

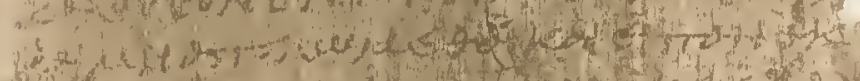

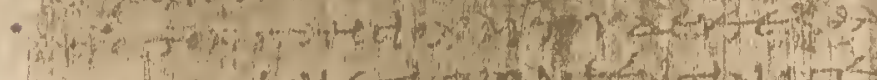

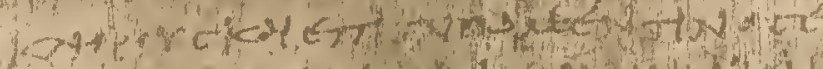

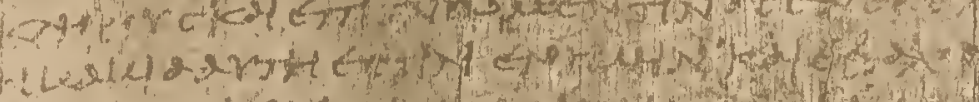
هor

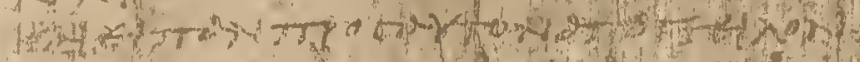

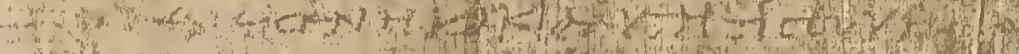

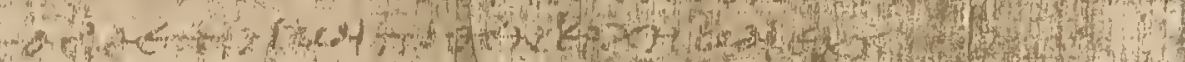

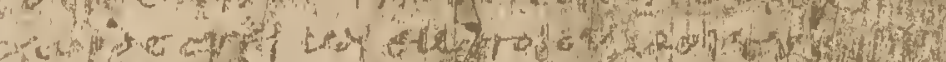

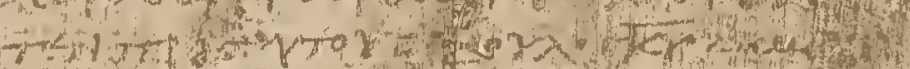

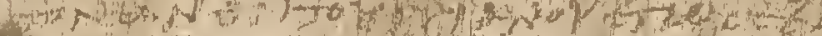

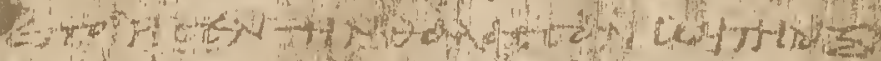

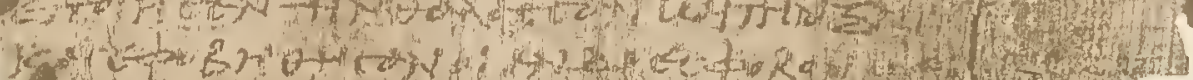

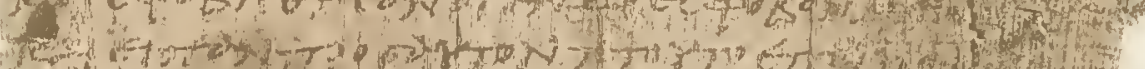

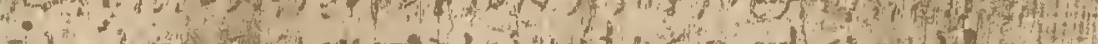

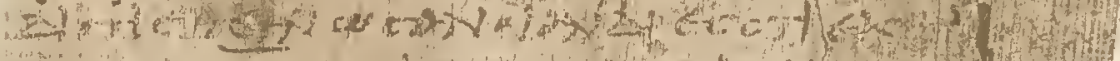

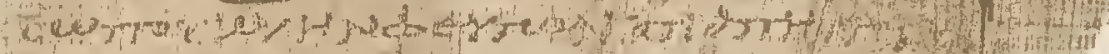

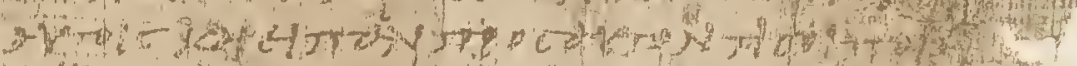
से w

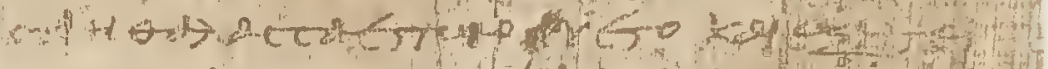

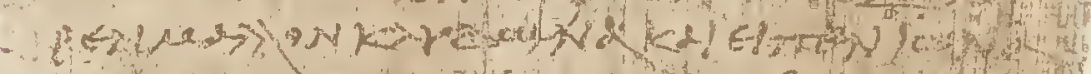

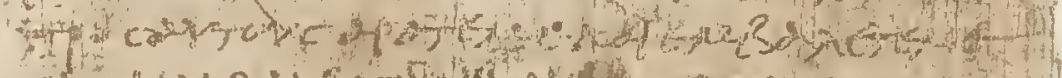

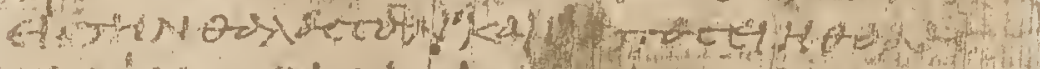

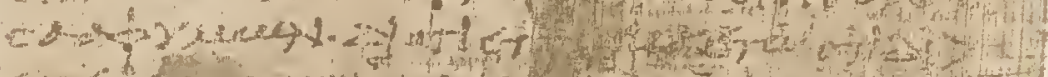

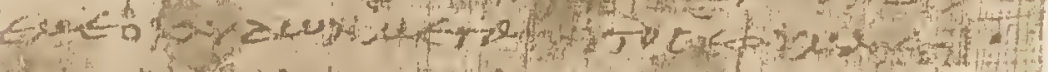

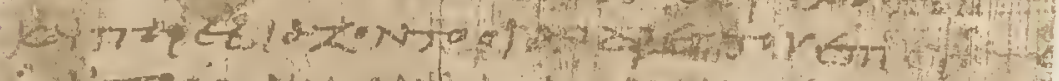

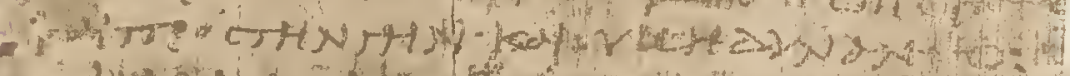

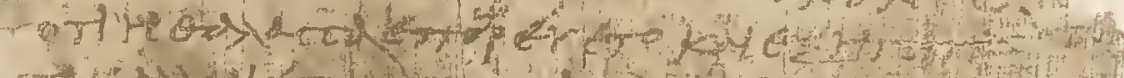

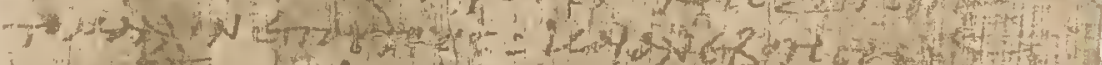

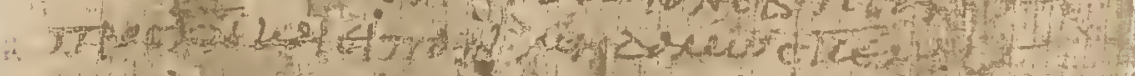

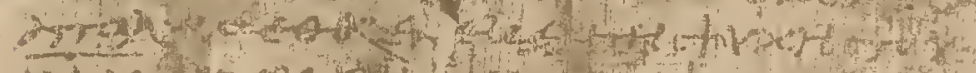

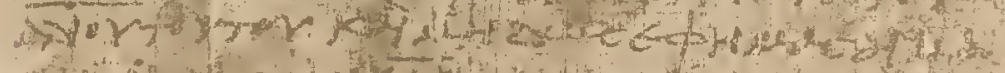

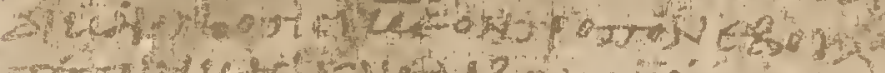

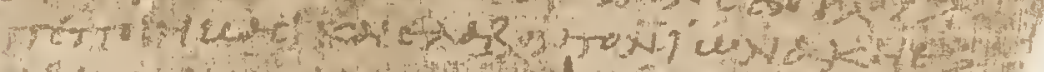

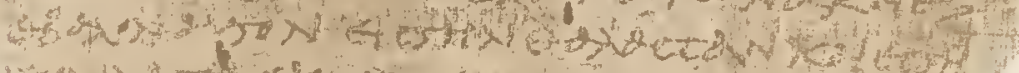

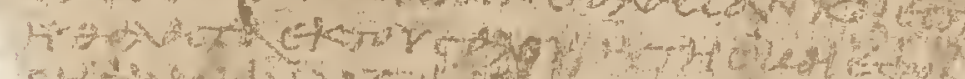

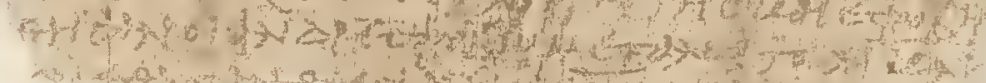

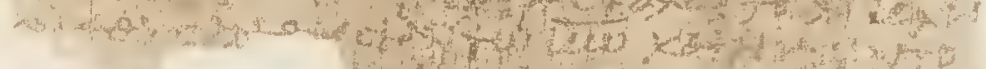

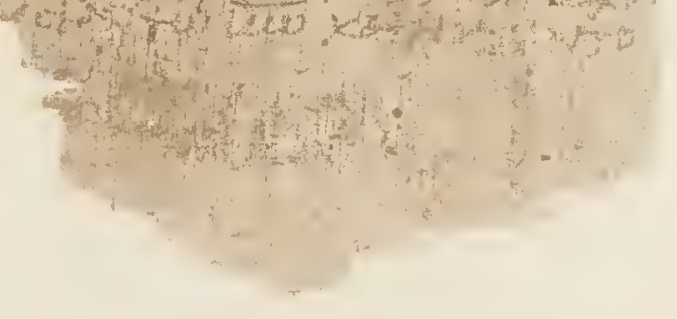




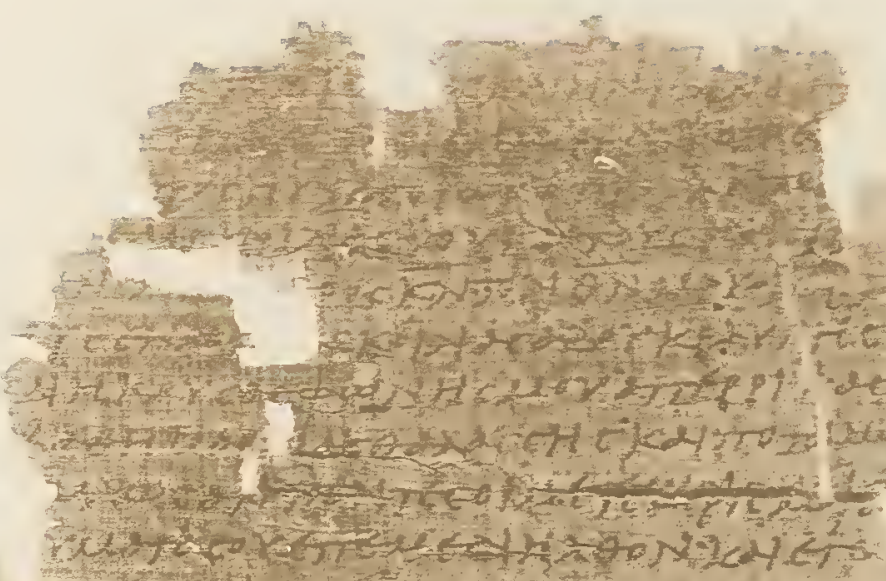

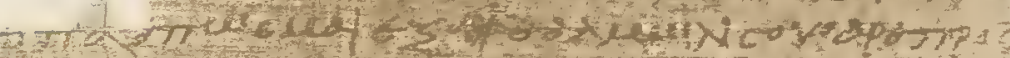

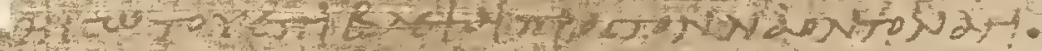

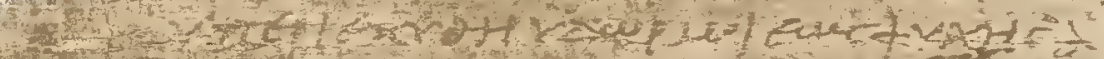

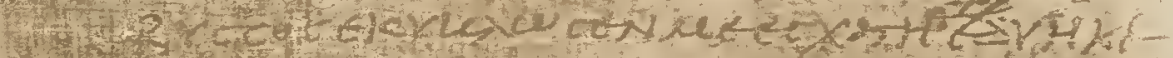

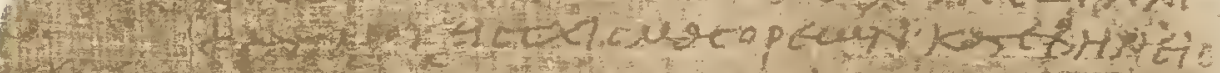

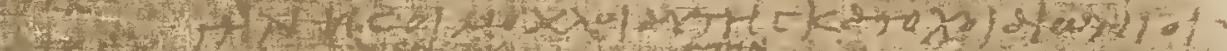

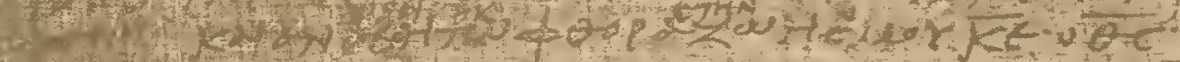

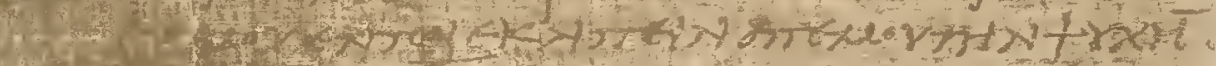

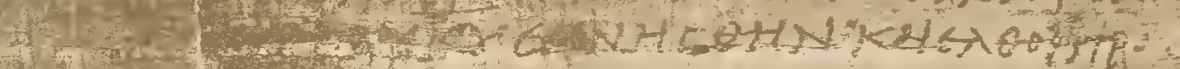

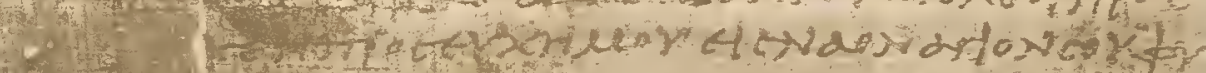

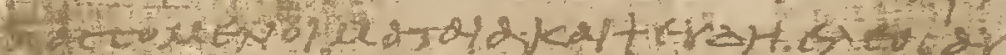

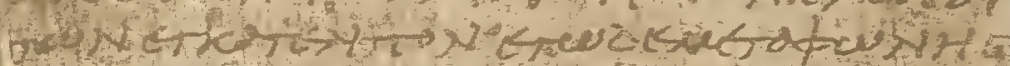

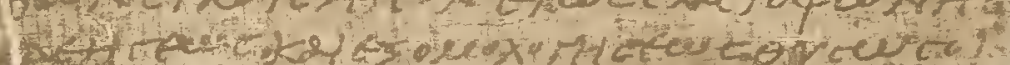

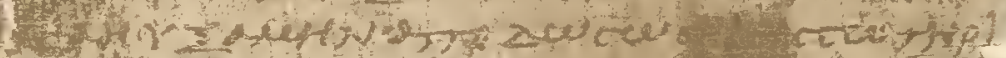

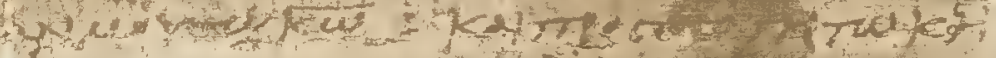

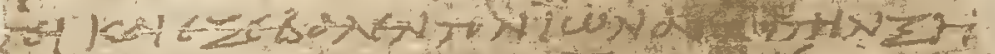

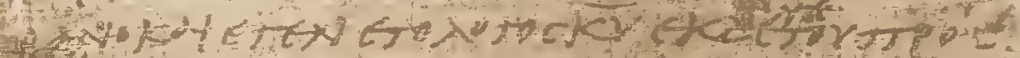

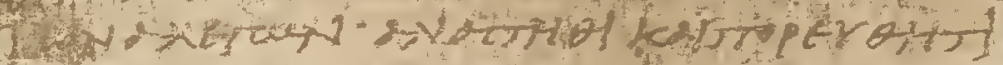

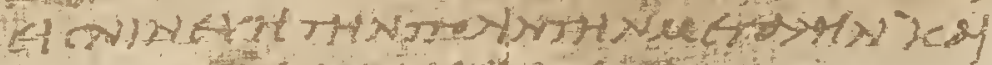

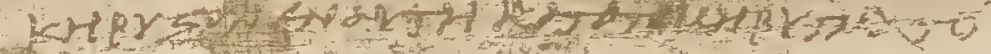

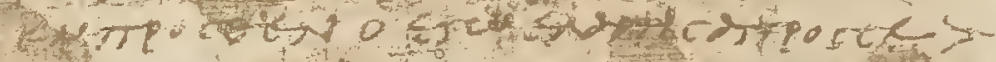

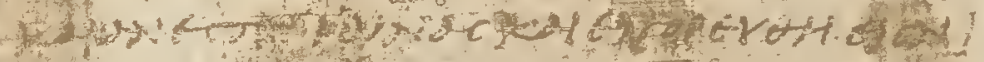

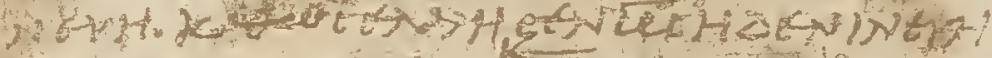
* Nग

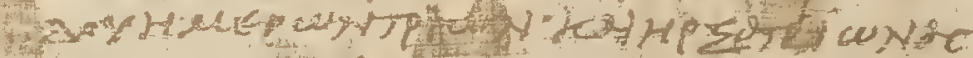

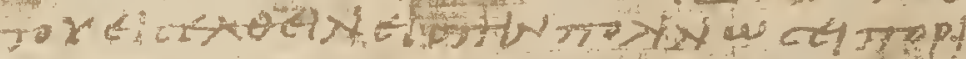

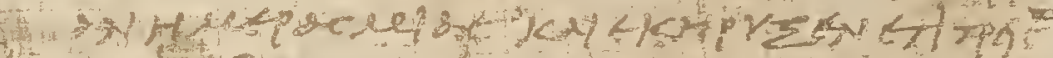

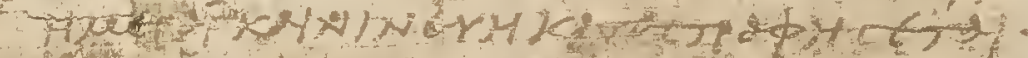

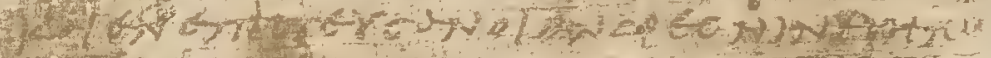

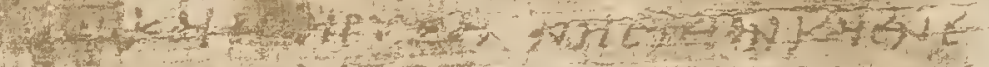

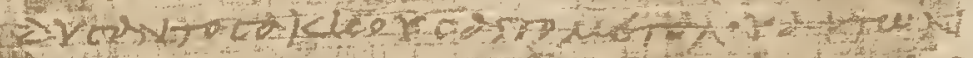

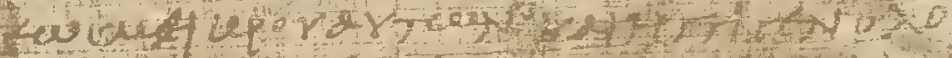

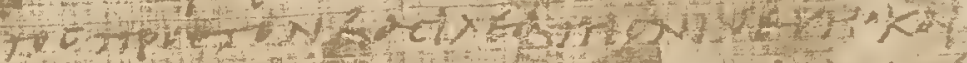

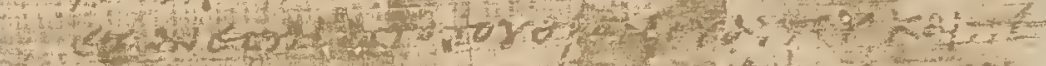

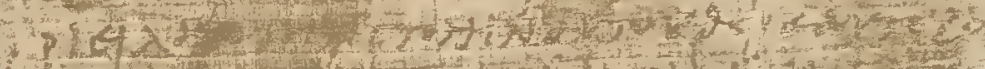

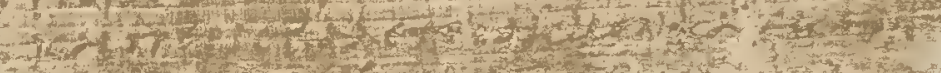

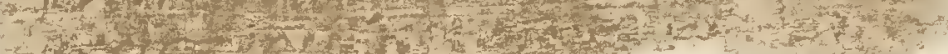

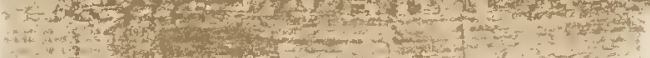

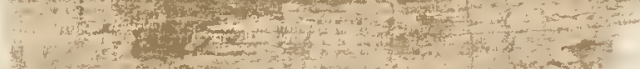

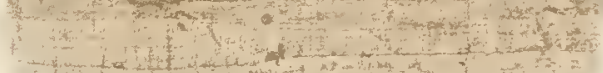

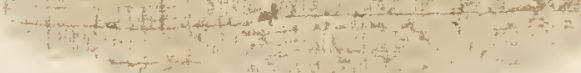

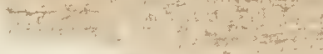
( 


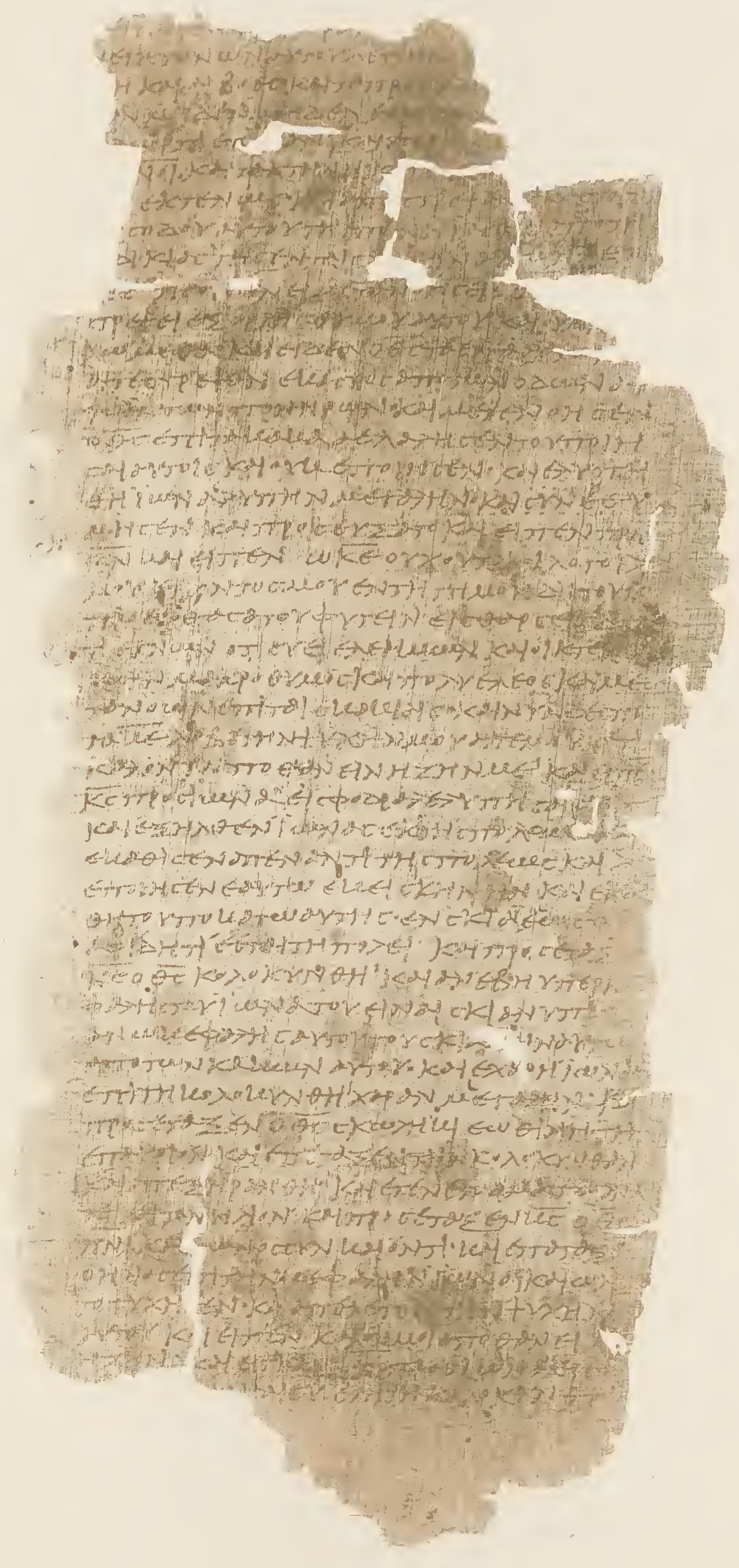

JOXAS iii, 7 -iv, 9 


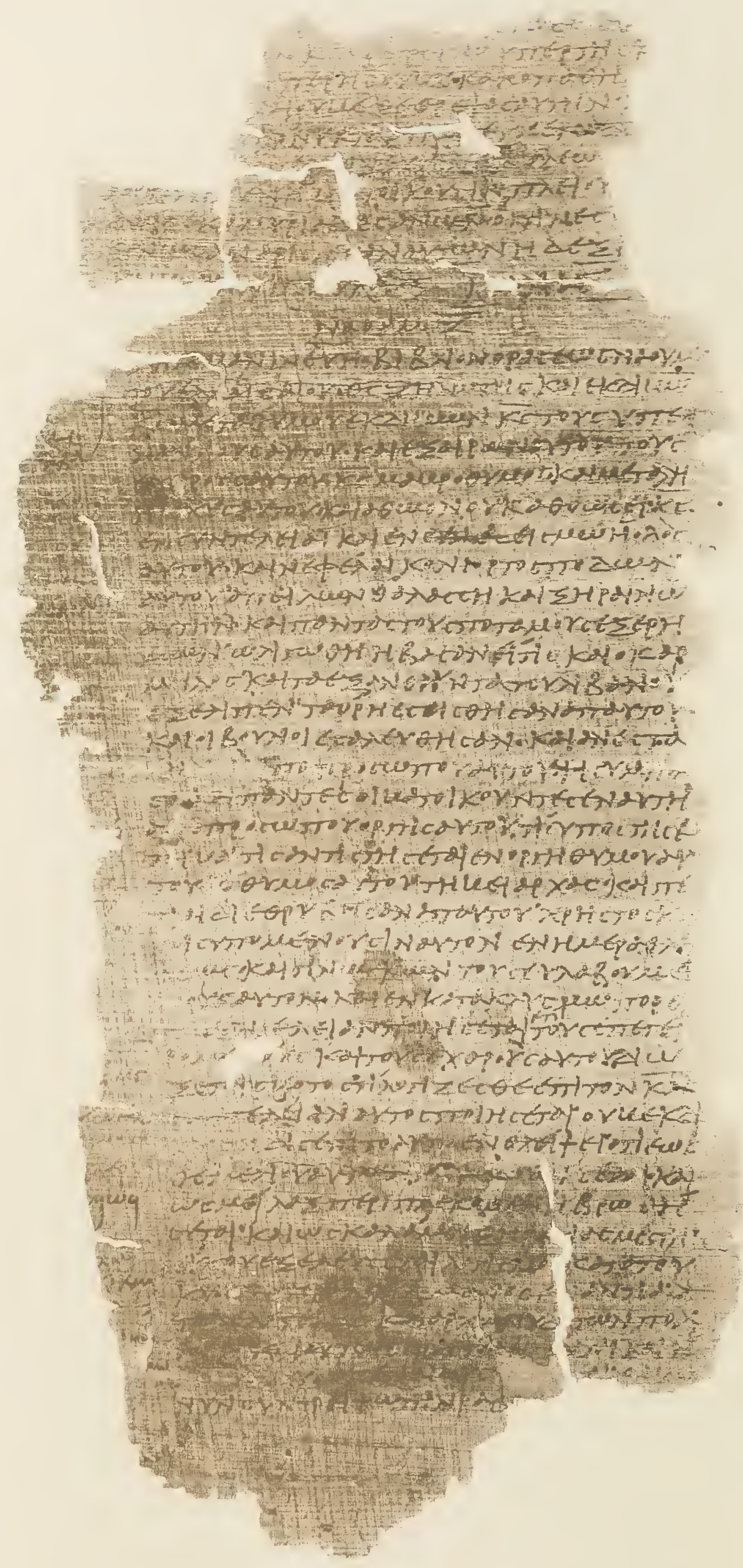

JoNas iv, 9-NAHCM i, 13. 


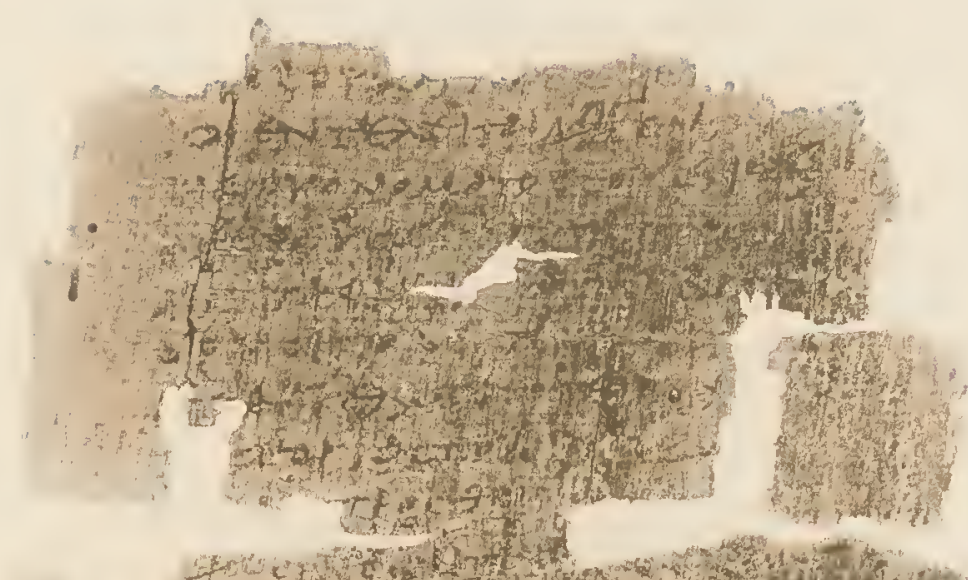

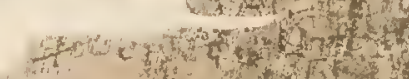

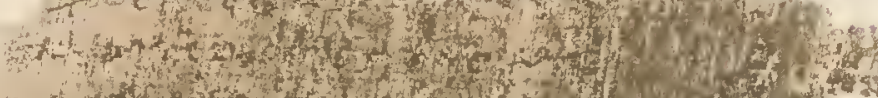

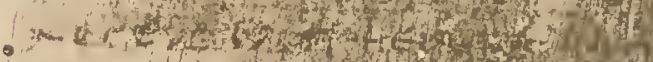

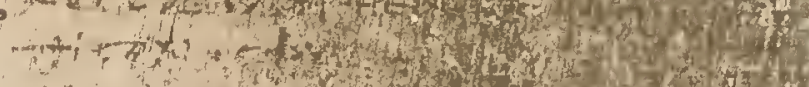

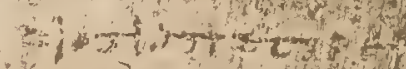

The

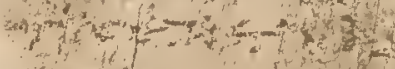

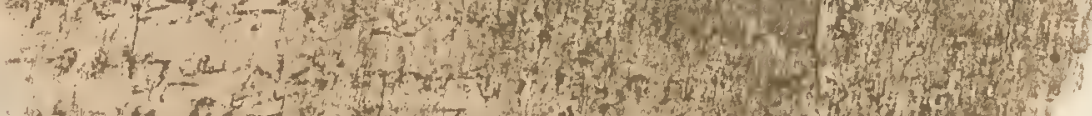

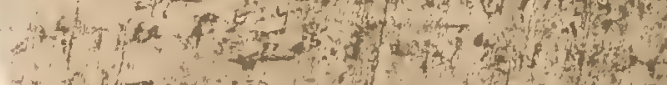

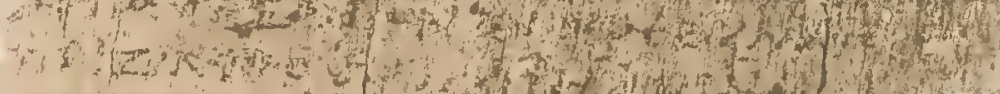

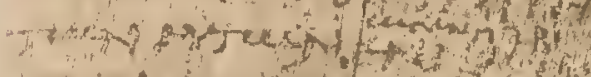

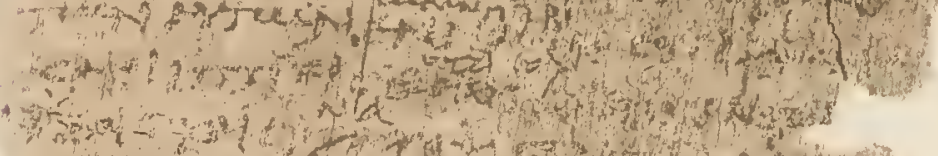

of

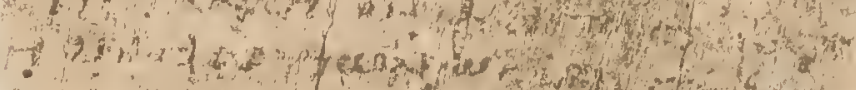

(c)

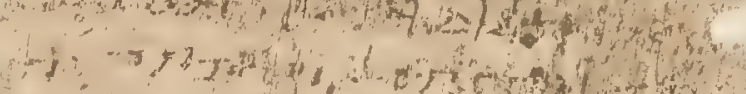

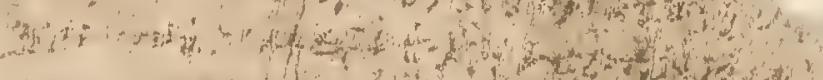

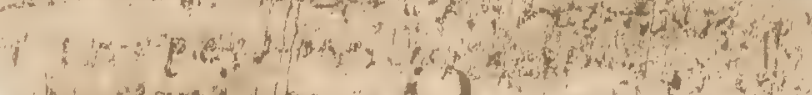

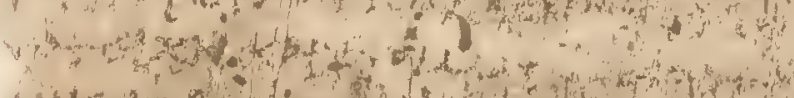

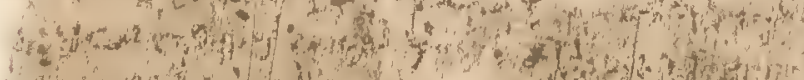

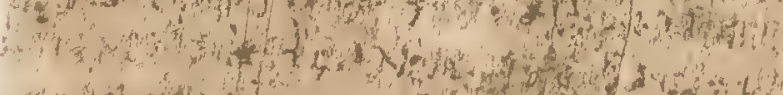

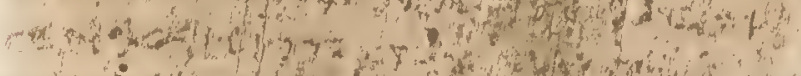

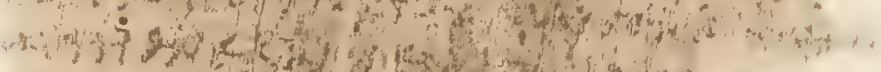

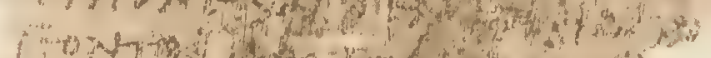

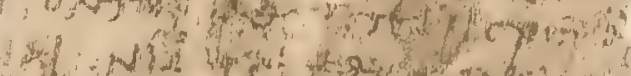

1. Not $20 x$ t

(1)

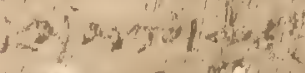

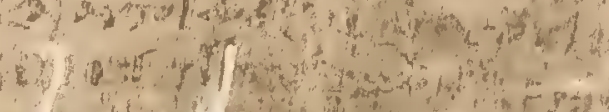

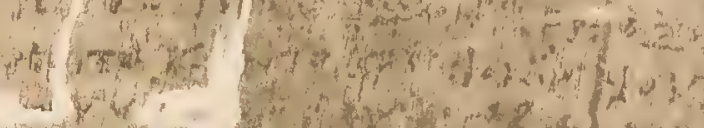

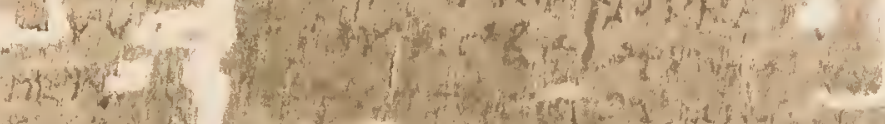

Herenting

instin

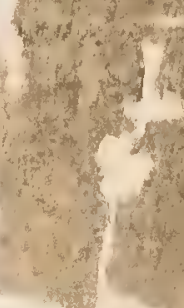




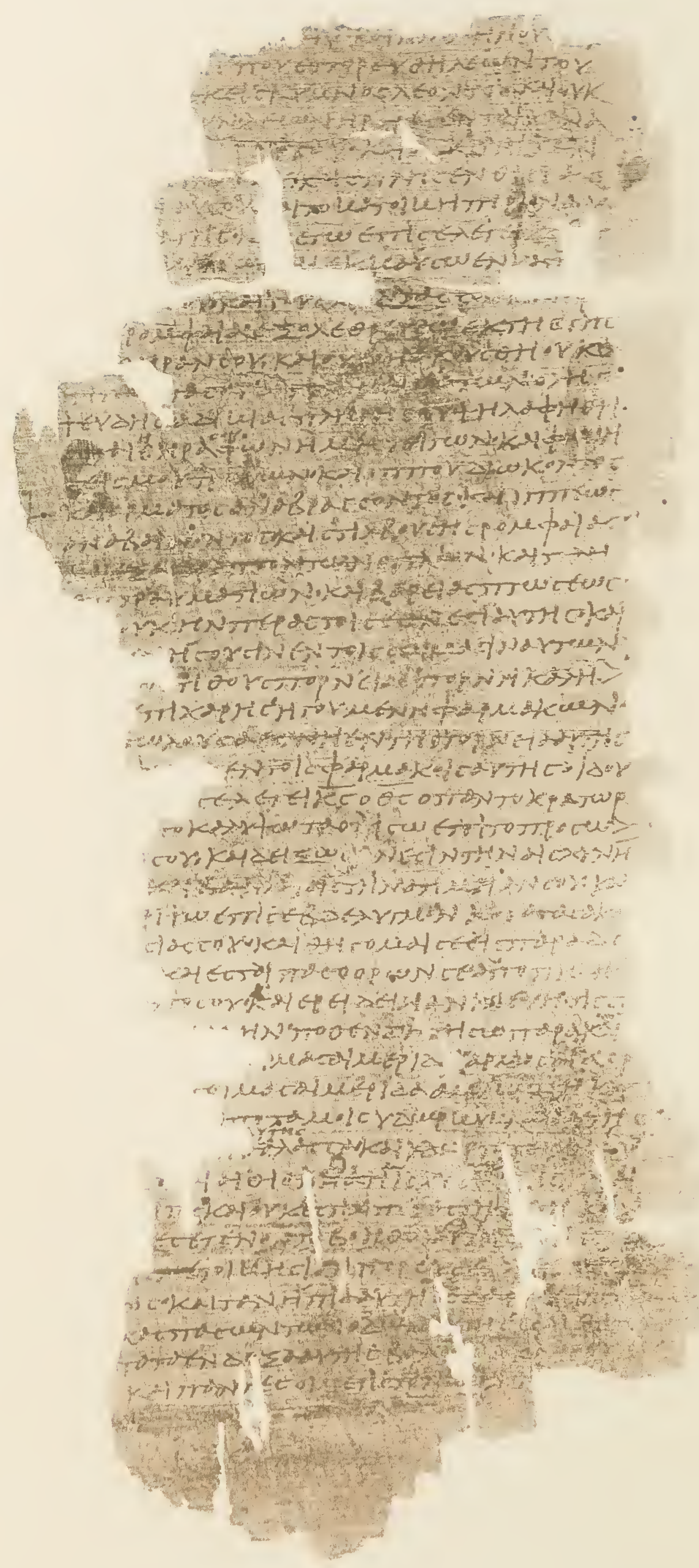

Nahum ii. 12-iii, 10. 


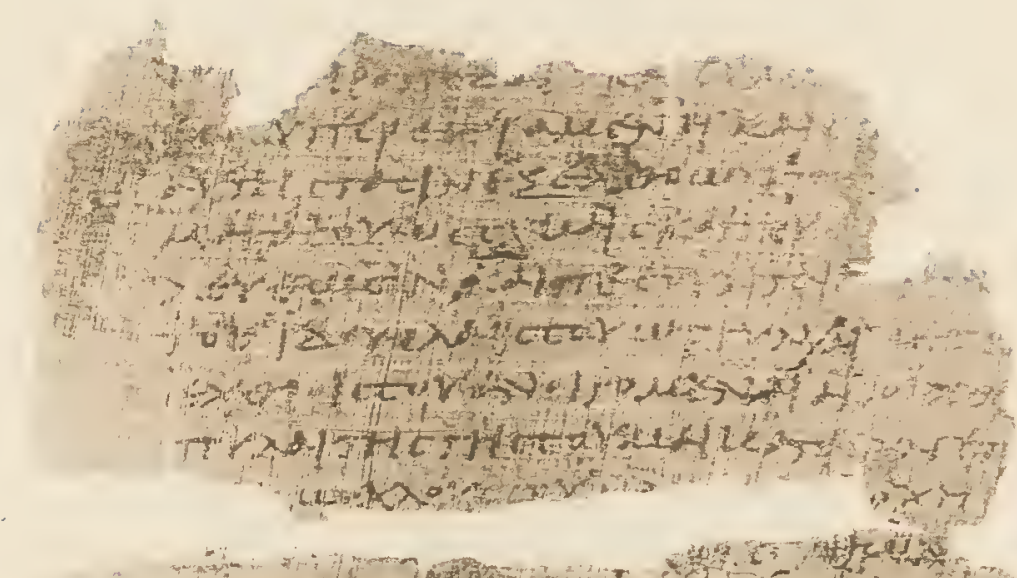

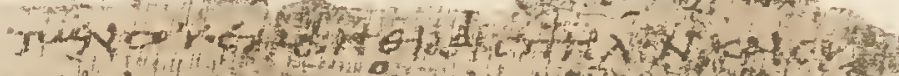

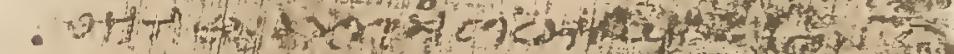

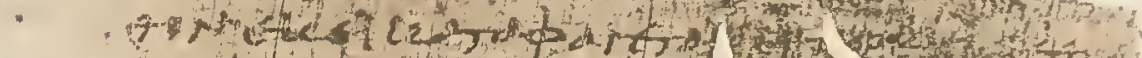

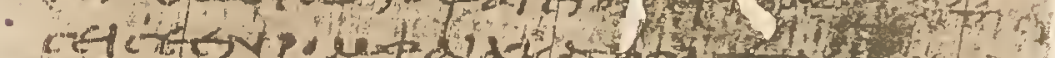

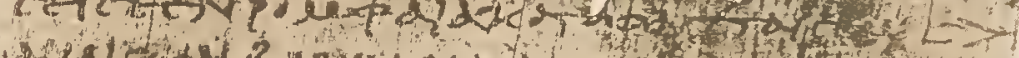

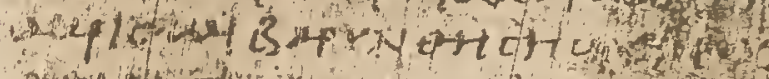

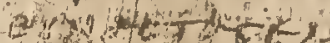

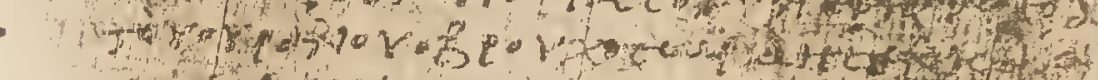

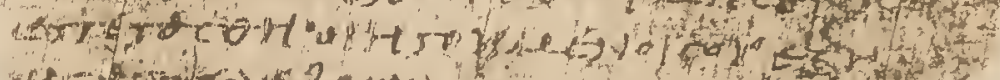

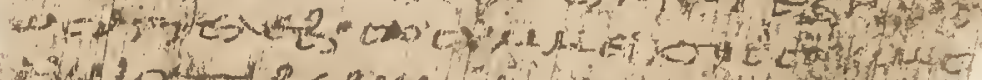

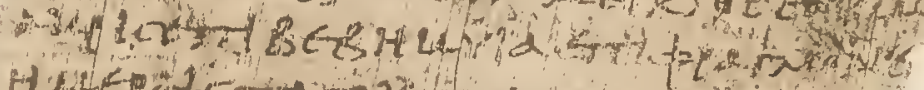

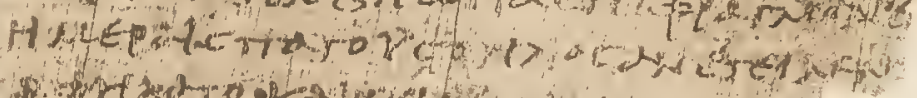

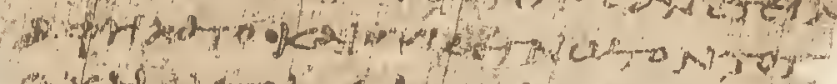

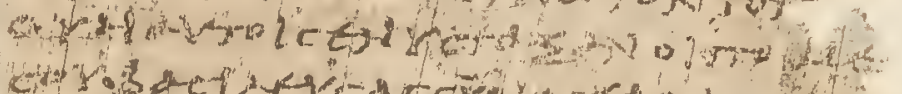

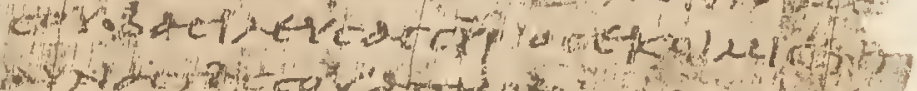

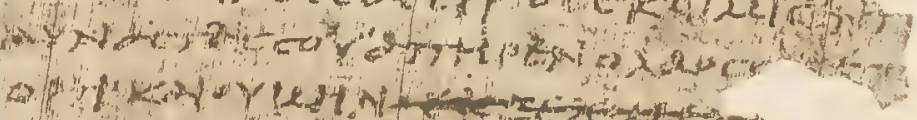

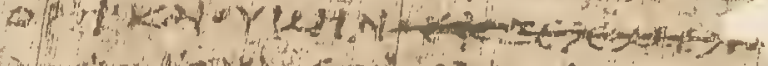

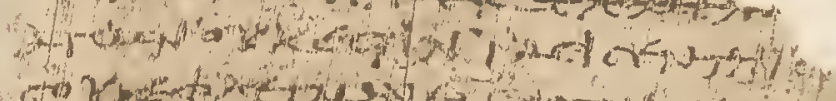

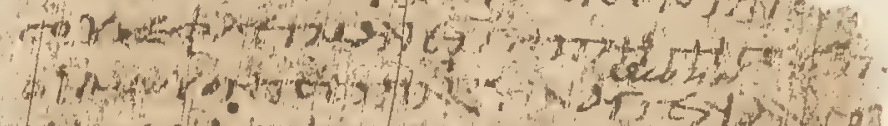

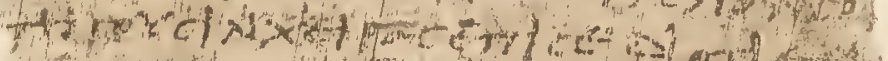

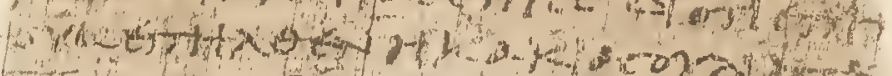

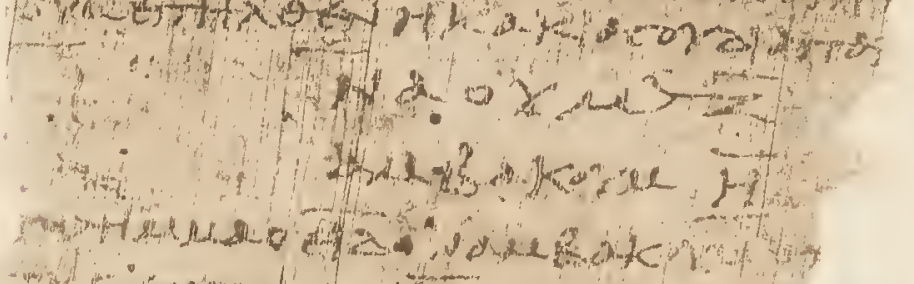

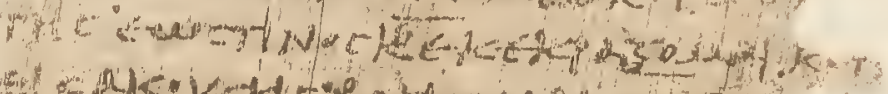

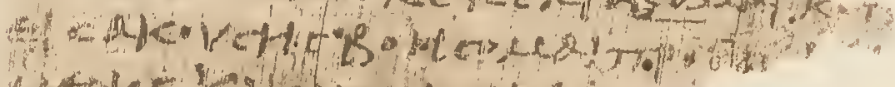

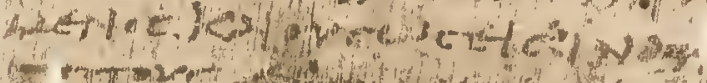

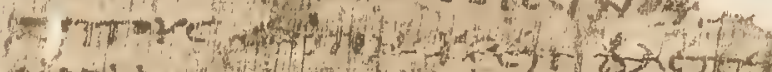

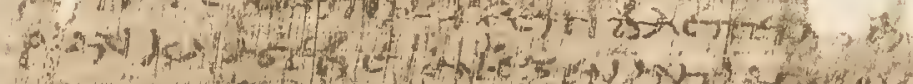

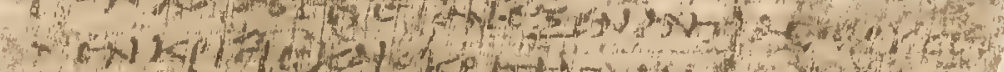

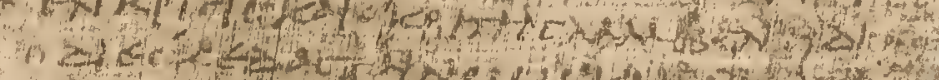

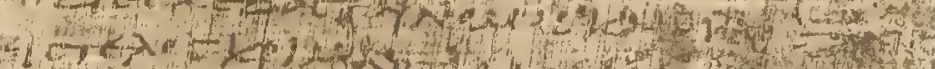

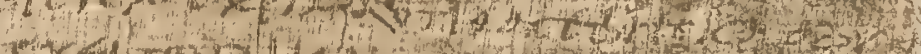

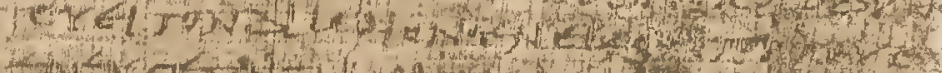

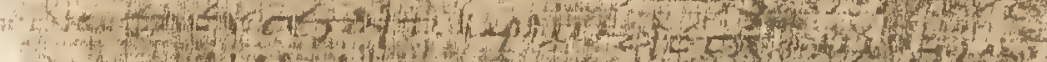

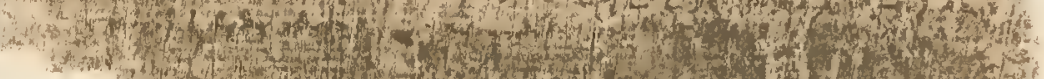

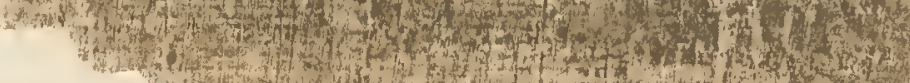

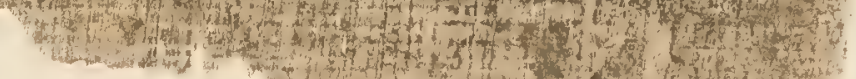
(5)

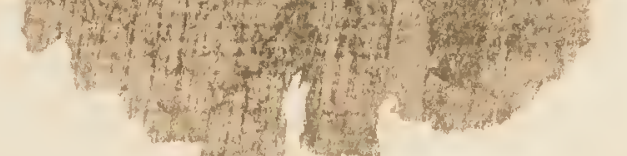




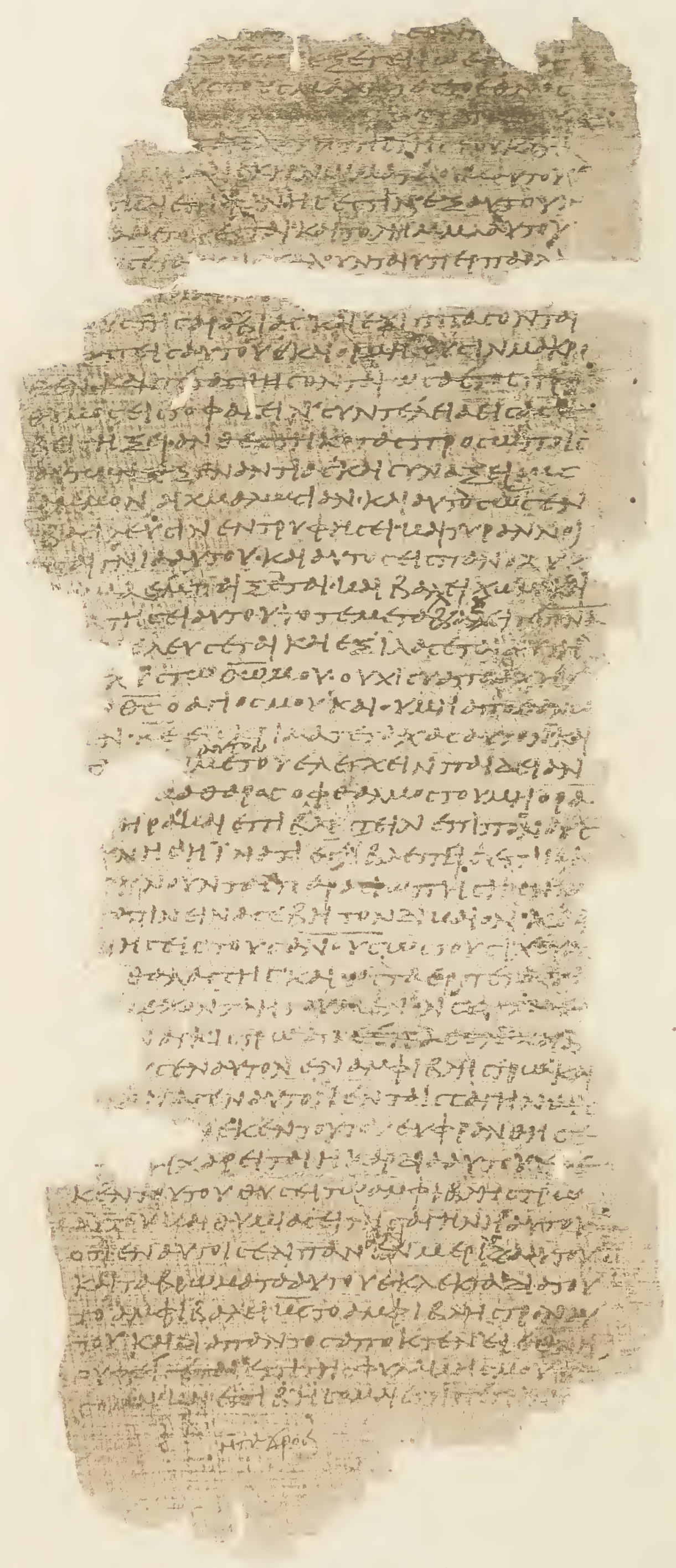

Habacte: i. 5 -ii. 1 . 


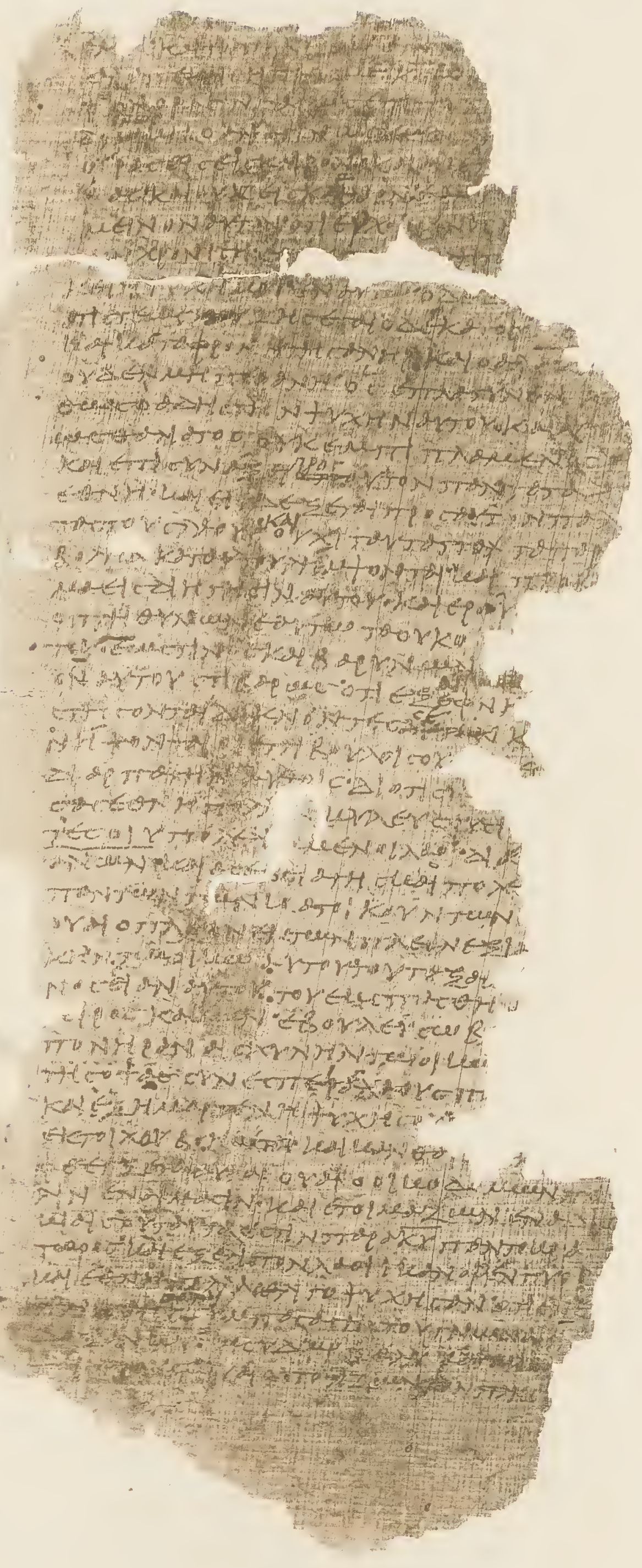

Habacte ii, 1 - 1.). 


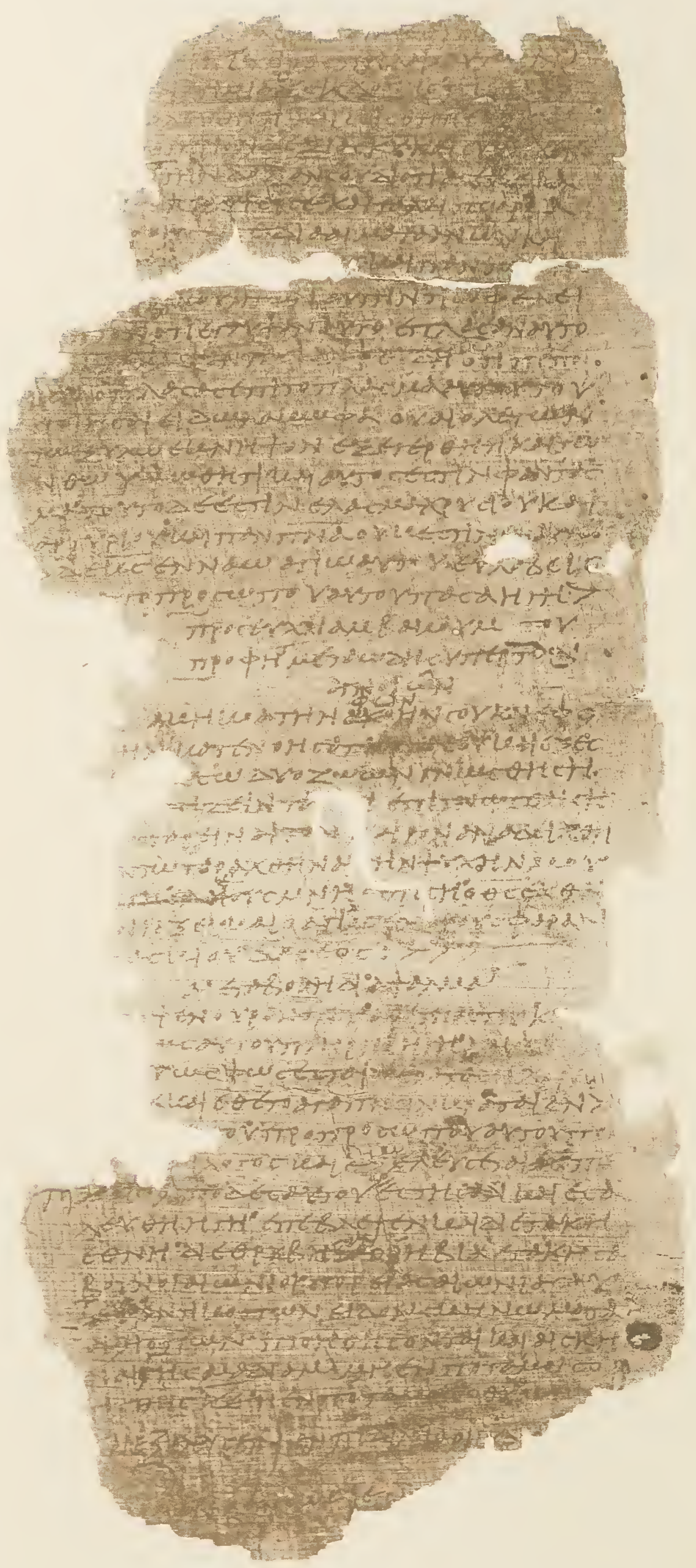

Habace: ii, 15 -- iii, 8. 

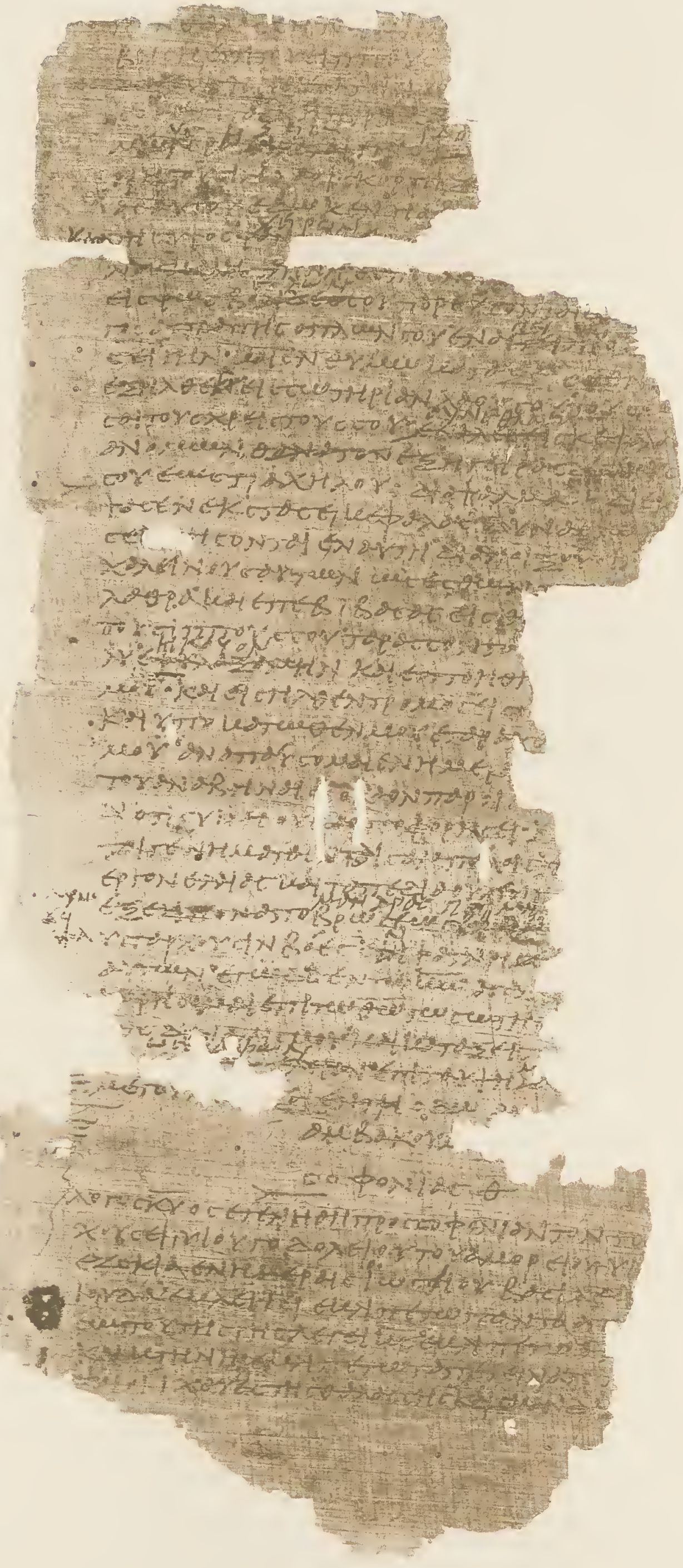

Habacle iii, 8 - Zemianias i, 3. 


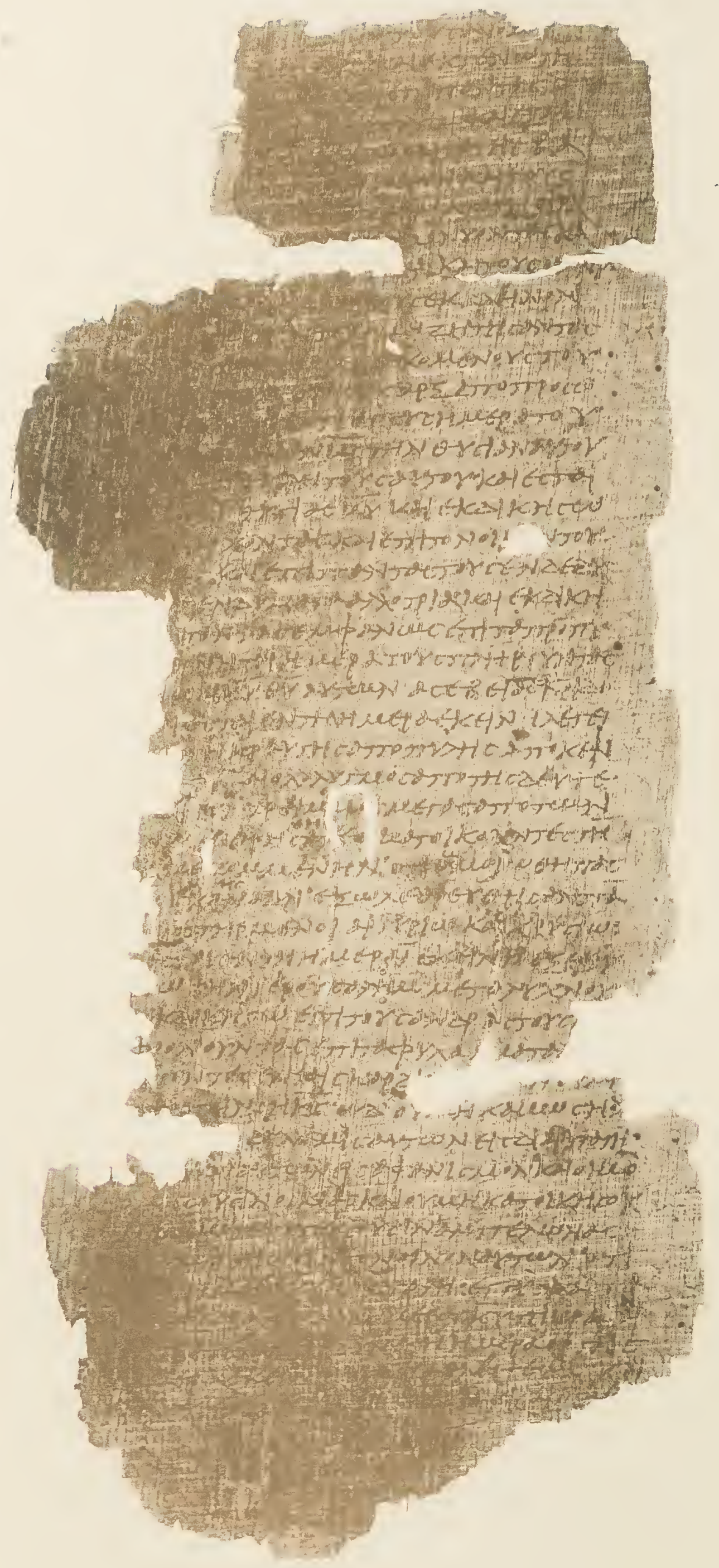

ZerHantas i, $3-15$. 


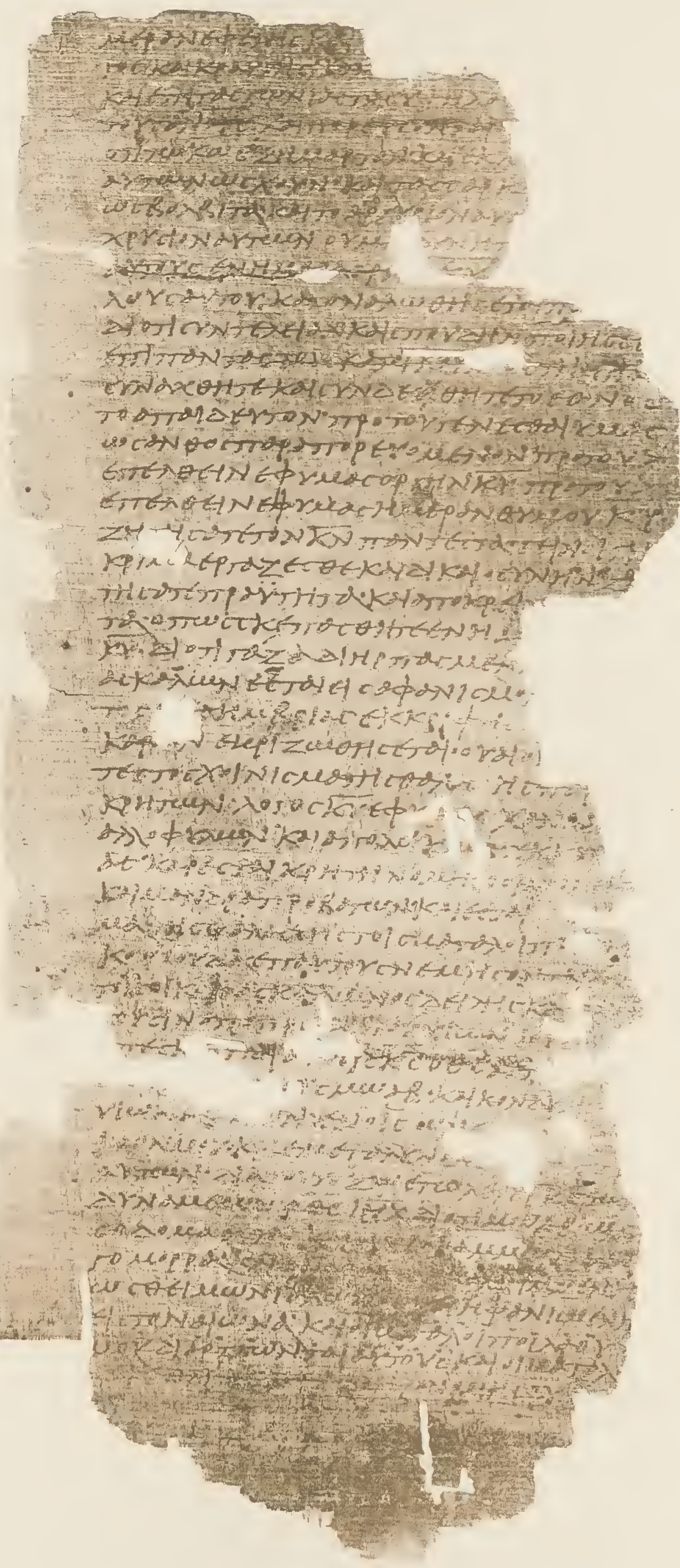




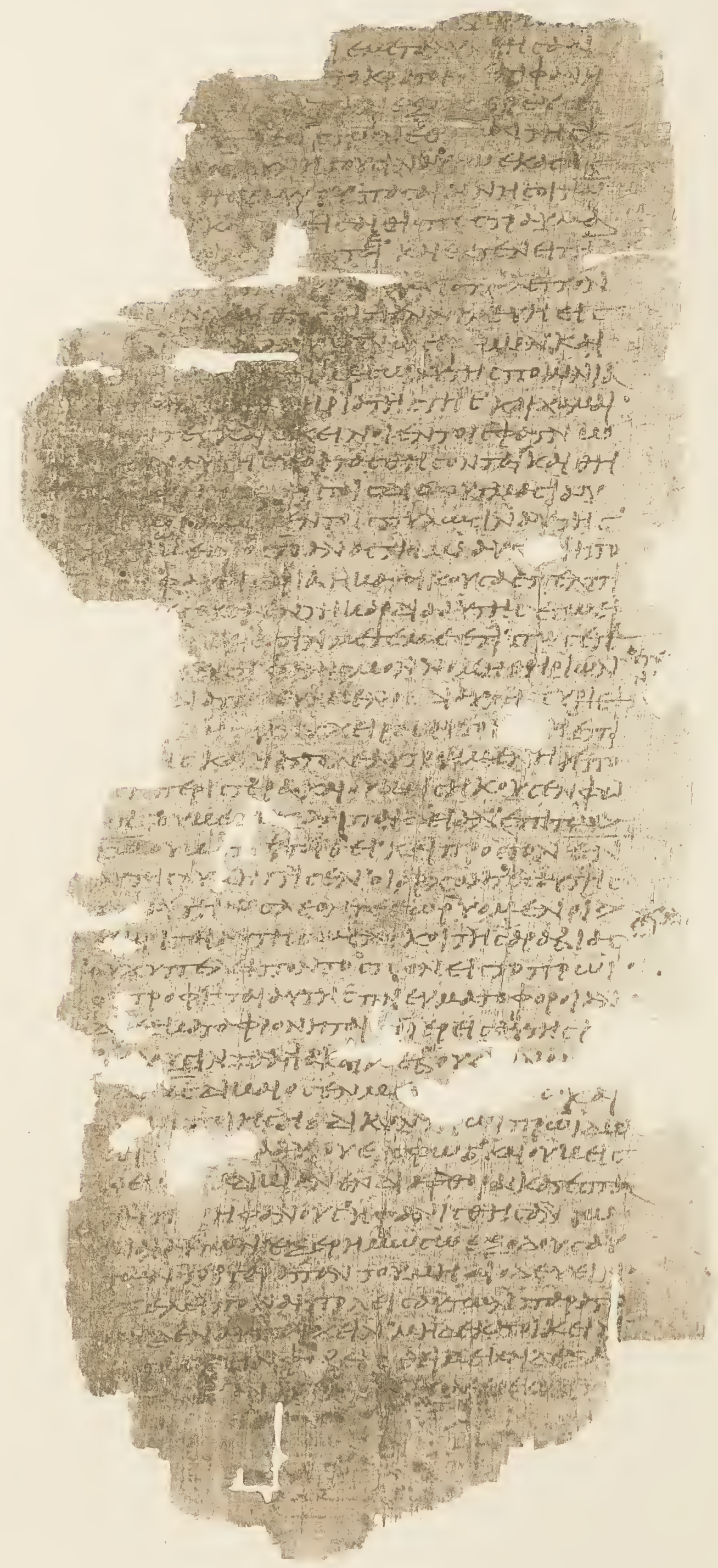

Zephanias ii, $9-$ iii: 7 . 


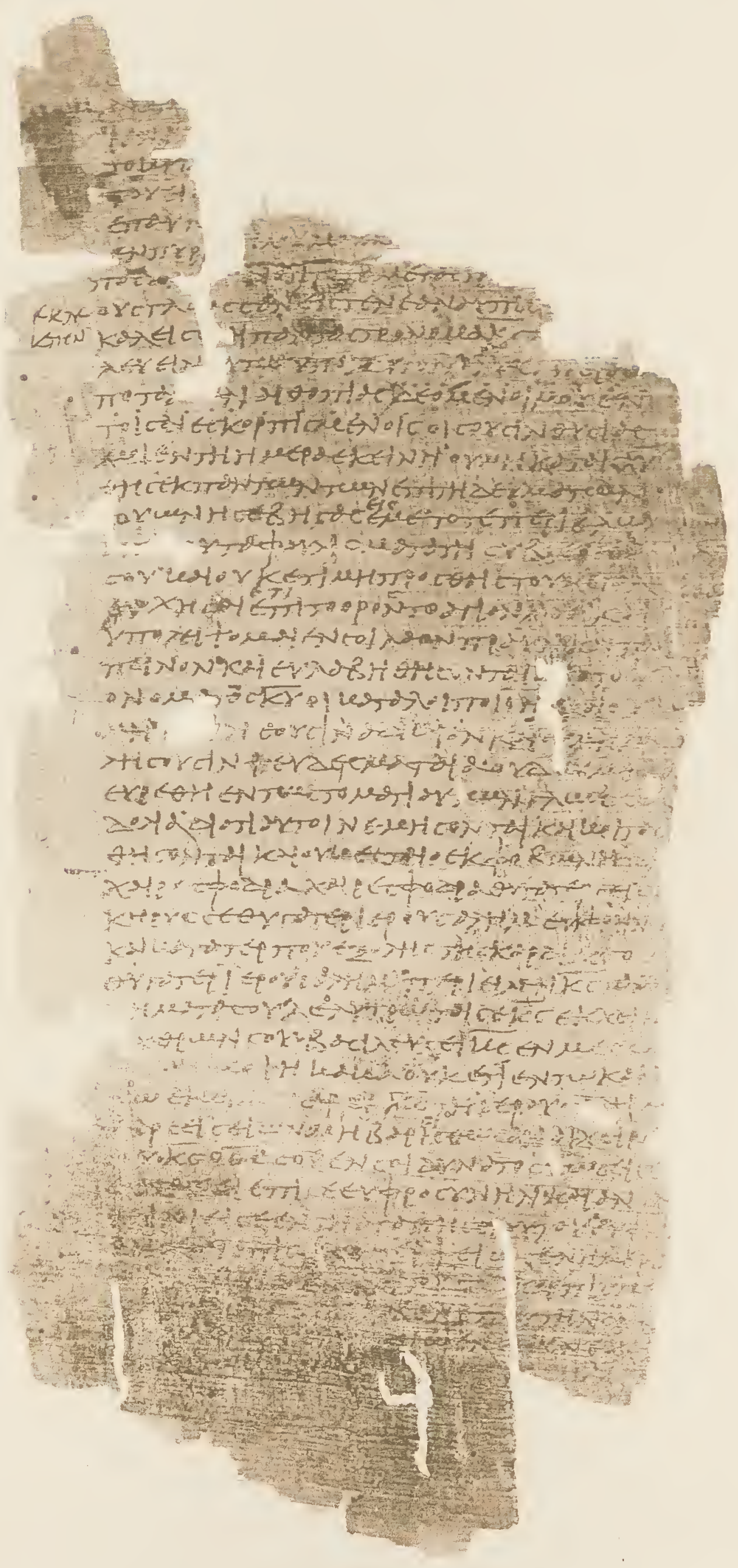




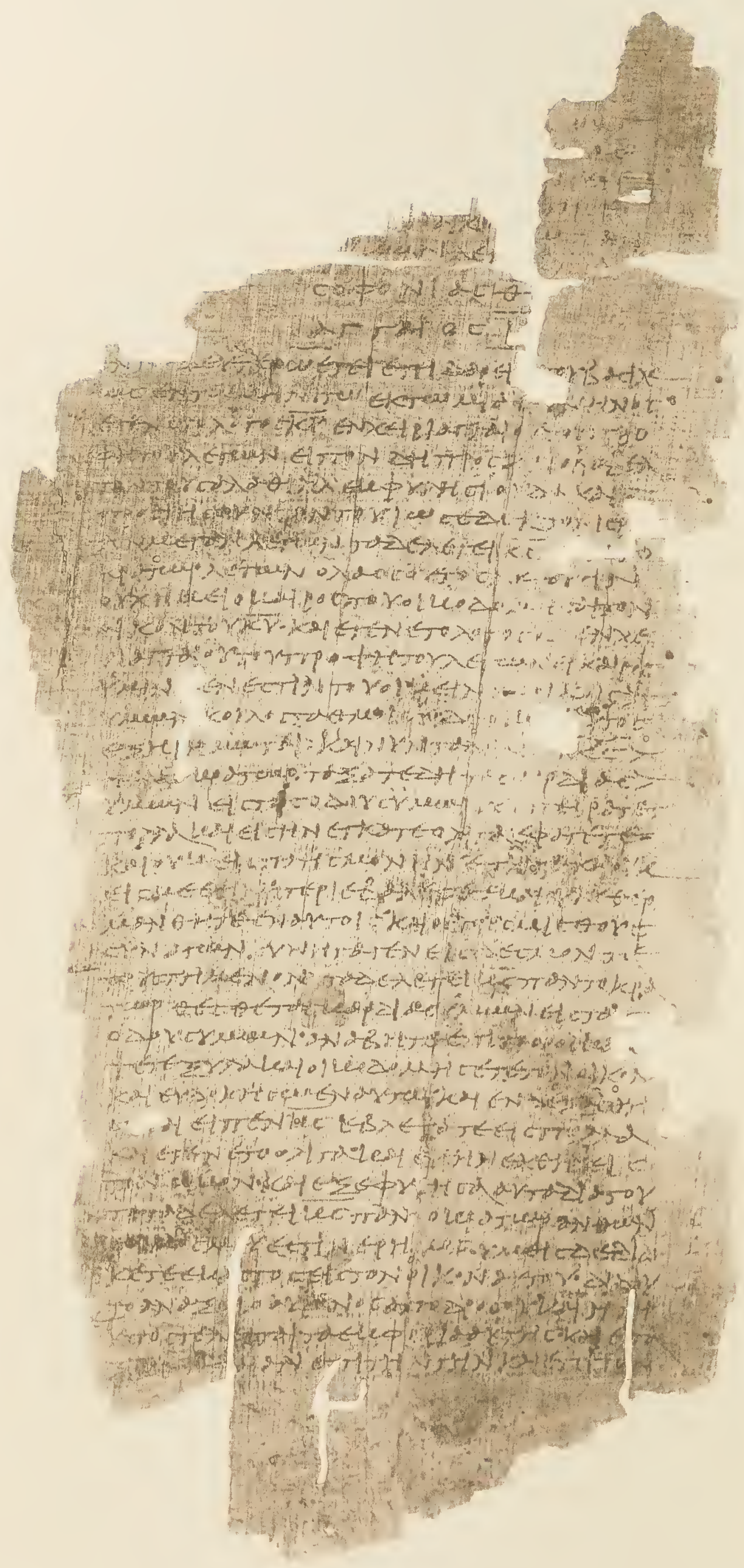




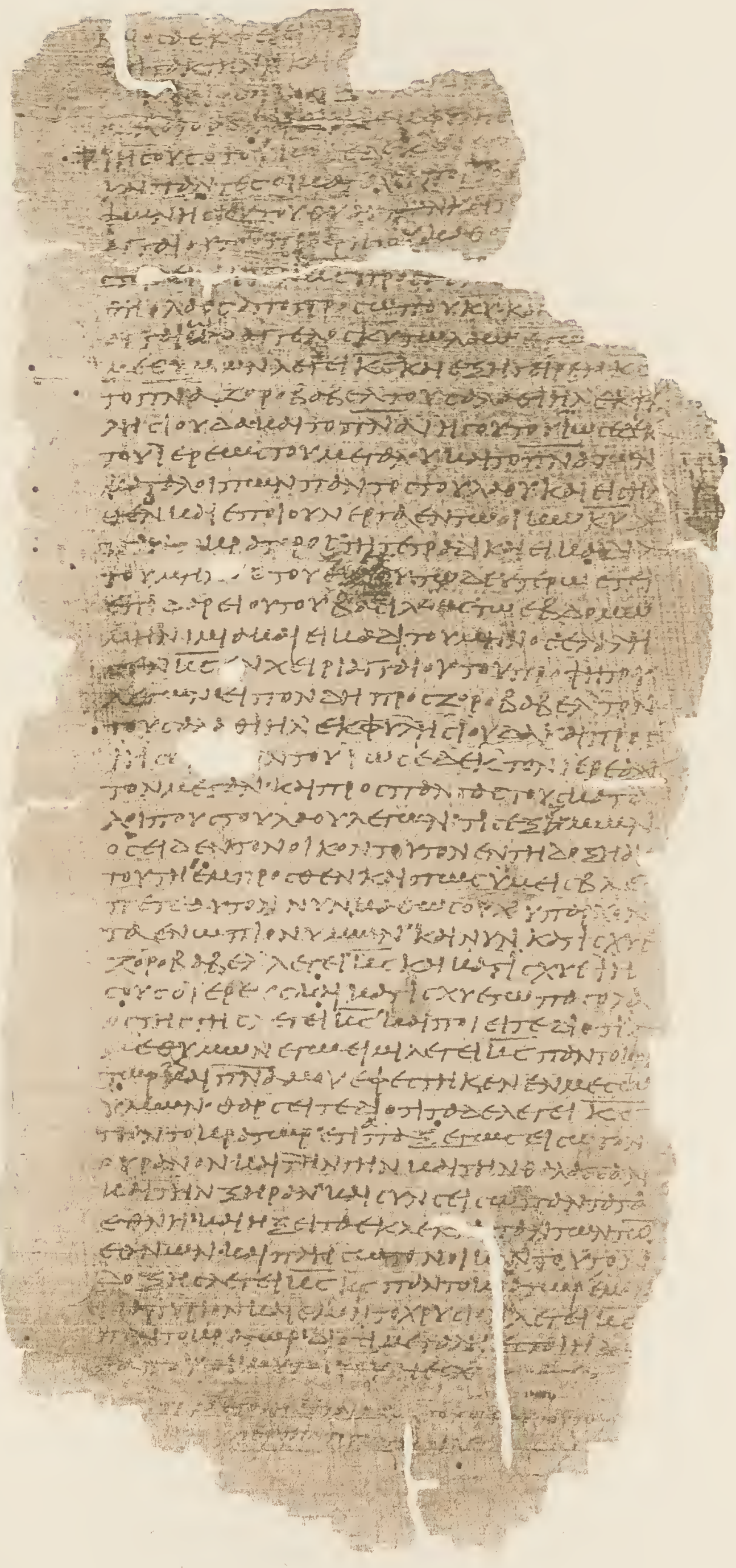

HAgG.AI i, 11 -ii, 9 . 

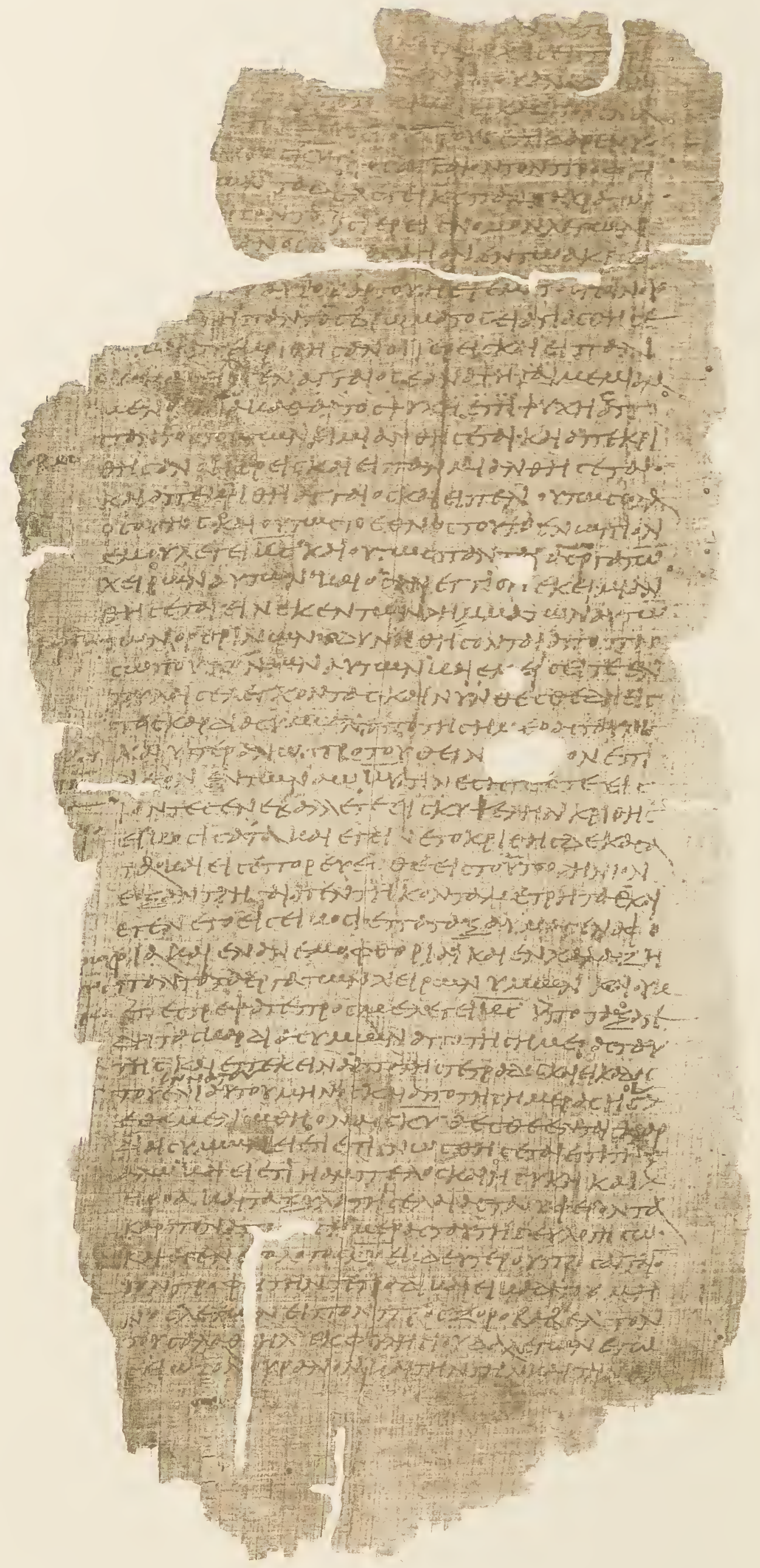

ILAGGat ii, $9-21$. 


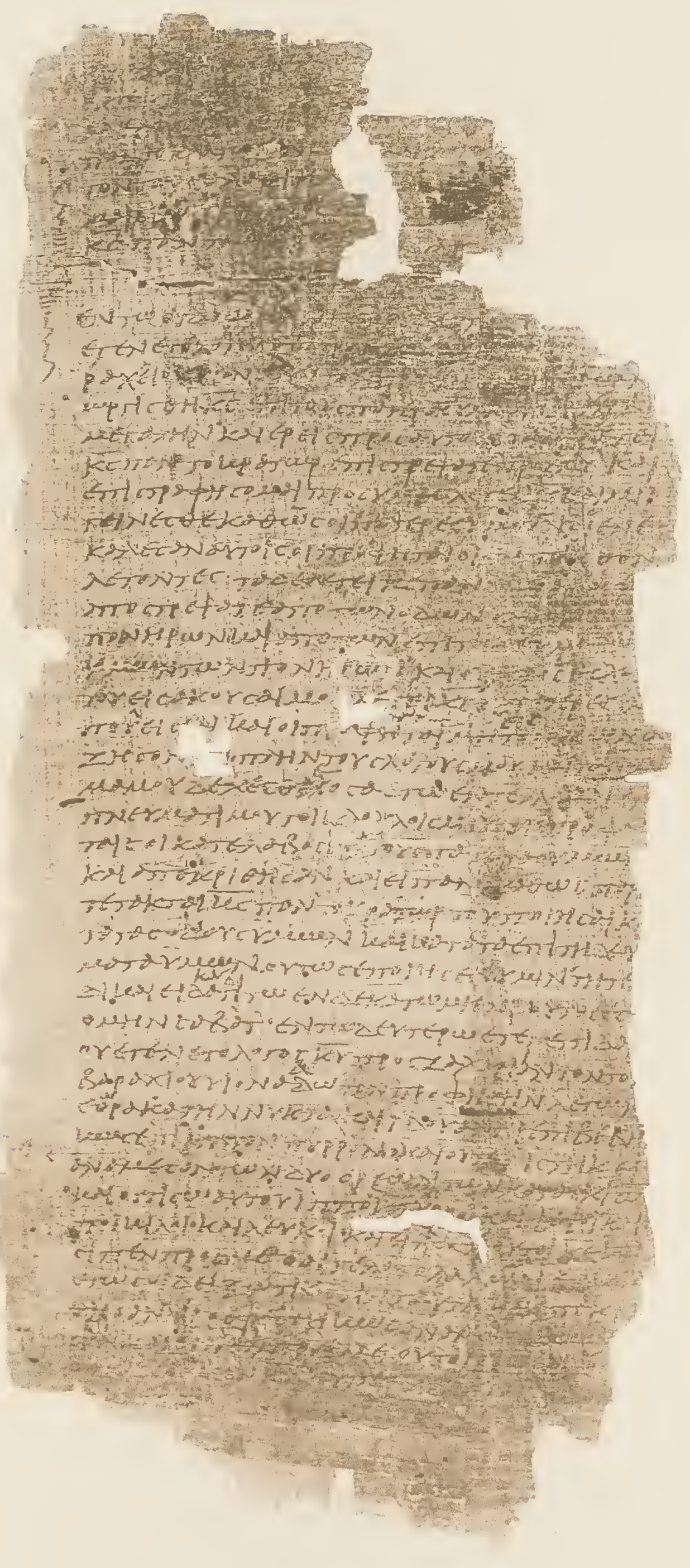

Uagigar ii, 21-J/Achuris i, 10 . 


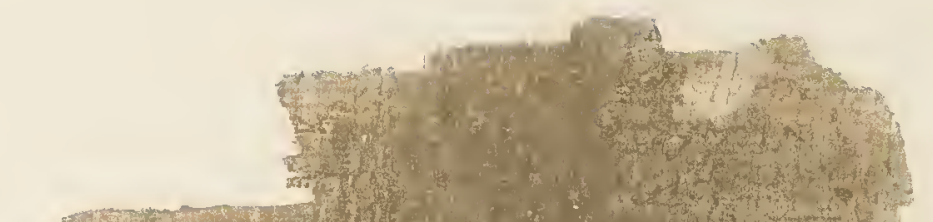

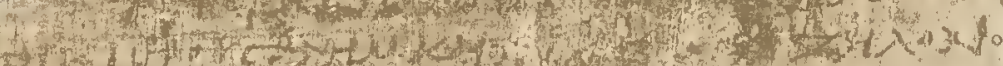

-

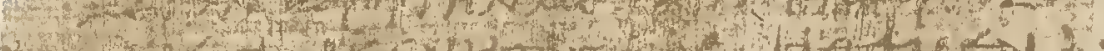

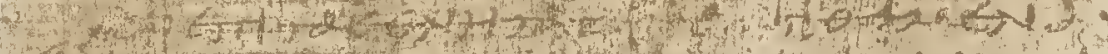

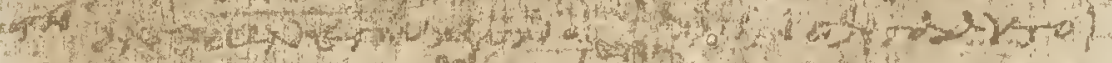

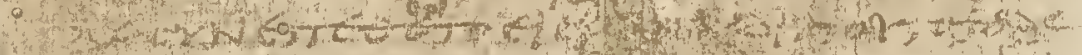

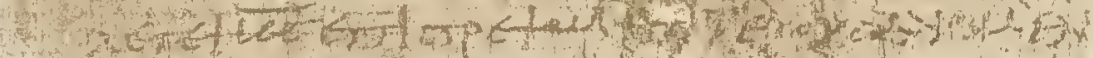

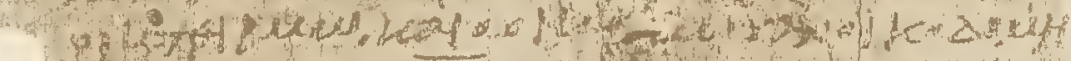

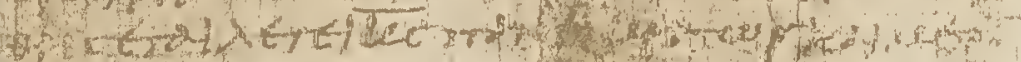

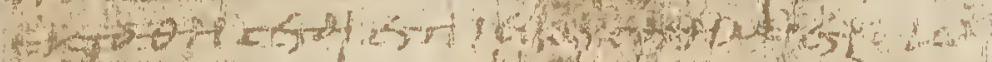

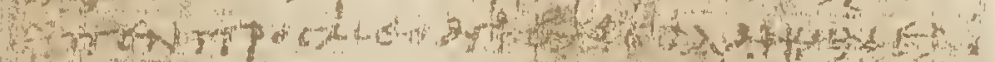

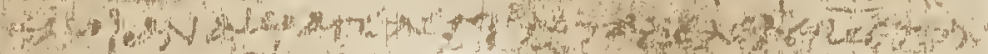

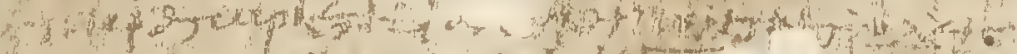

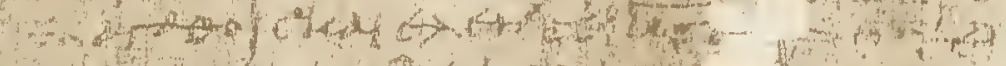

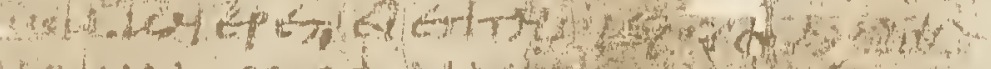

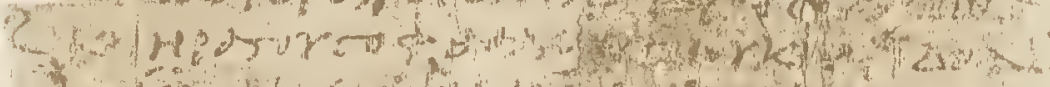

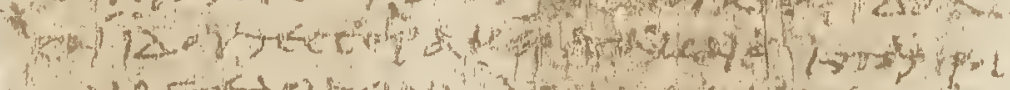
WNorr.

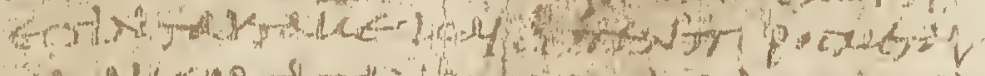

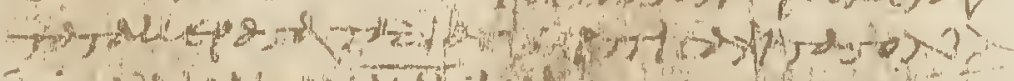

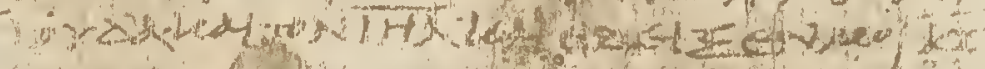

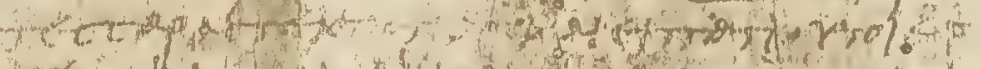

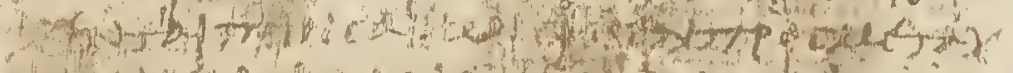

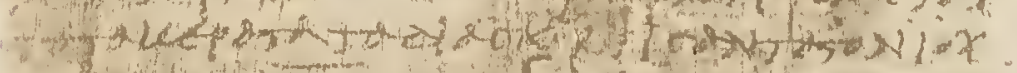

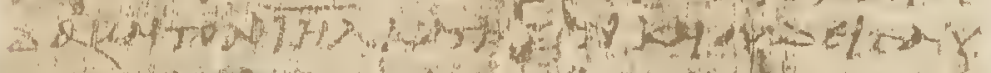
- dof

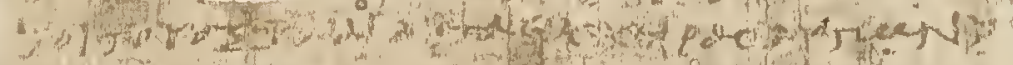

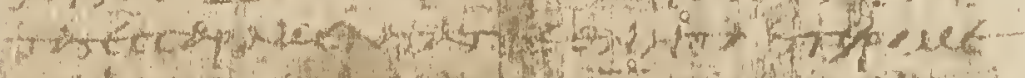

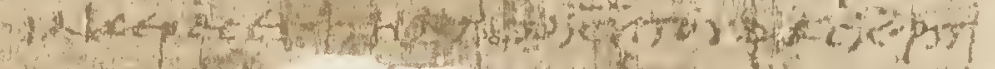

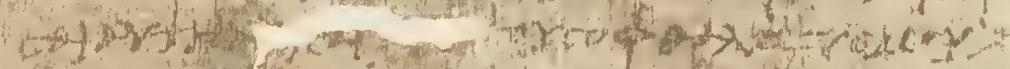

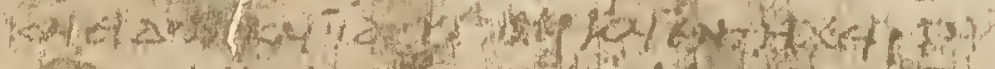

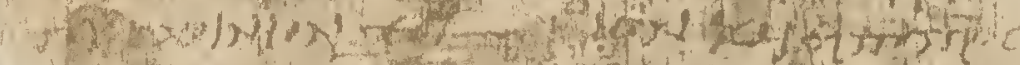

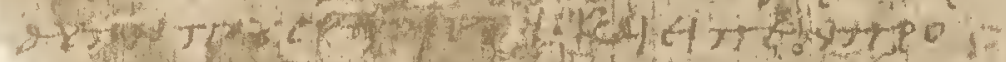

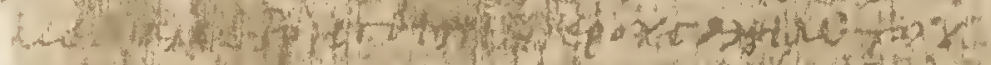

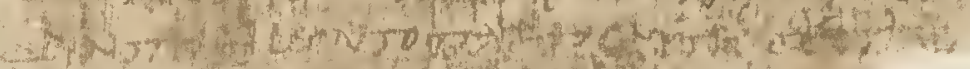
(4)

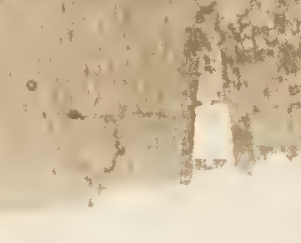




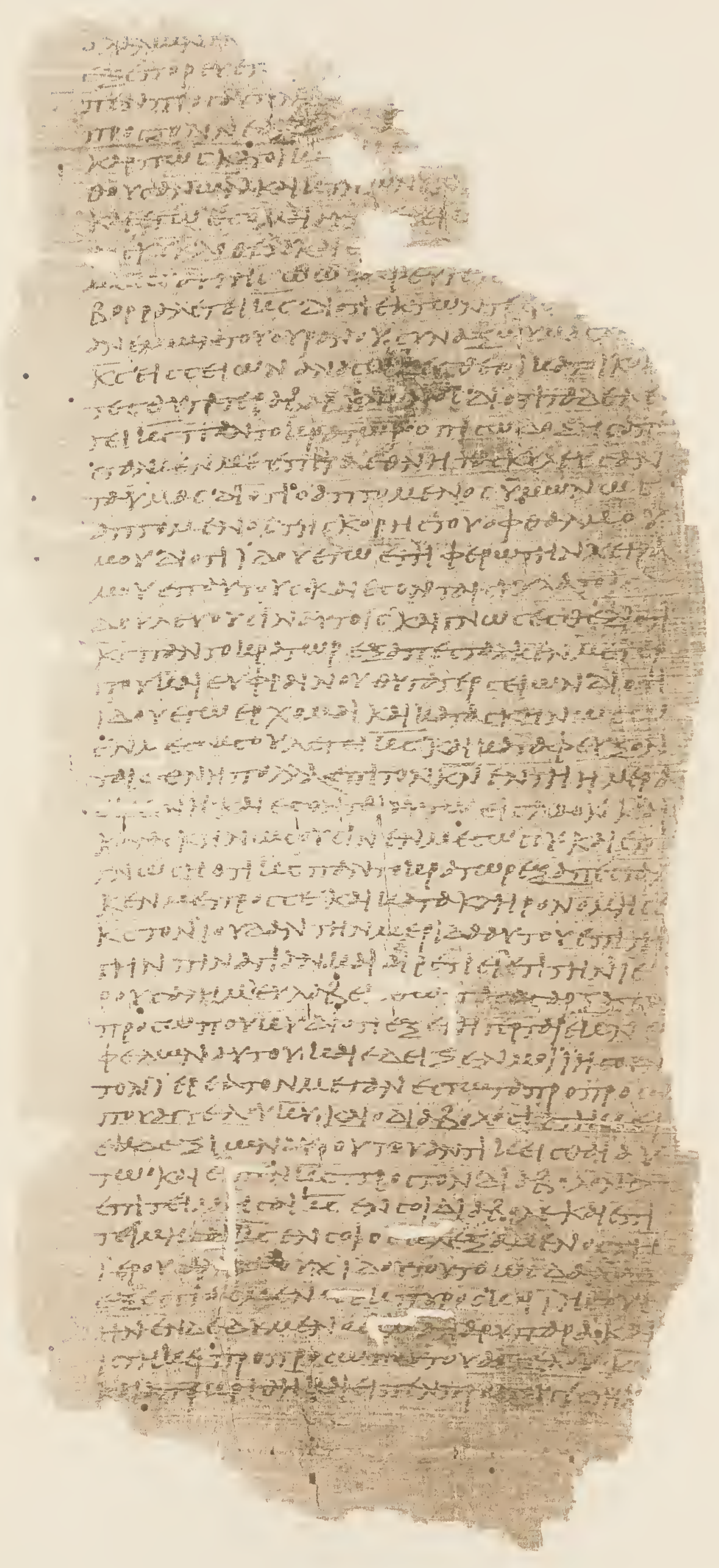

Zachartas ii, 2 - iii, 4 . 


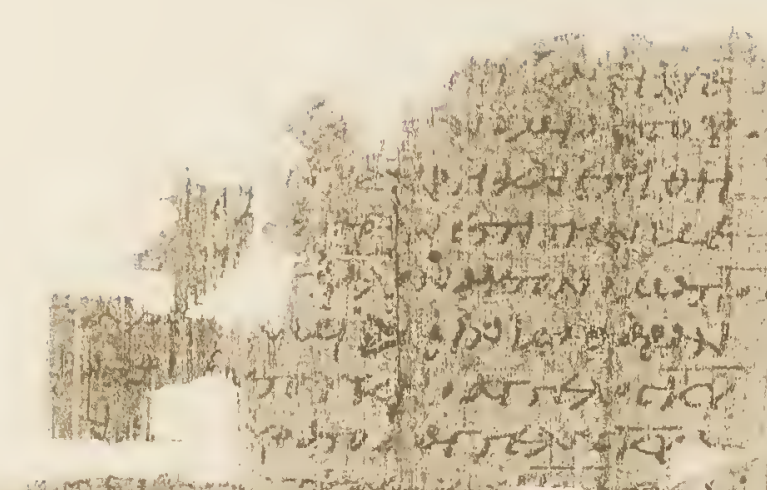

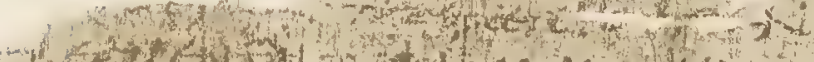

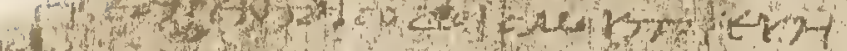

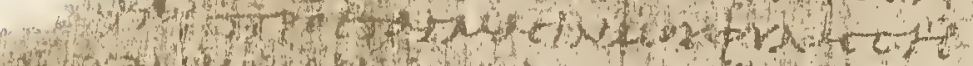

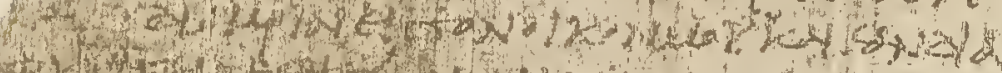

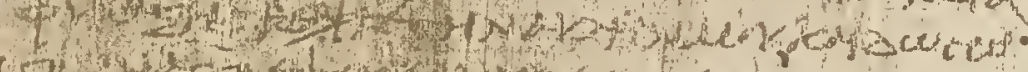

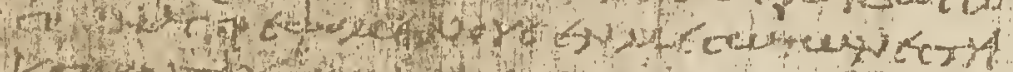

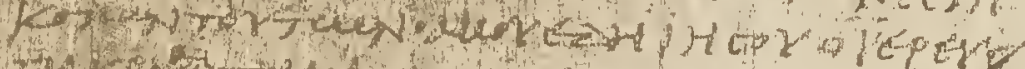

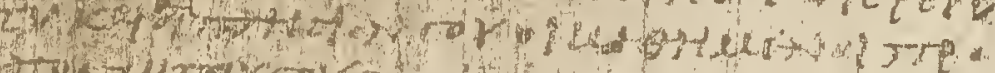

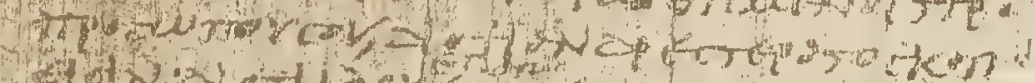

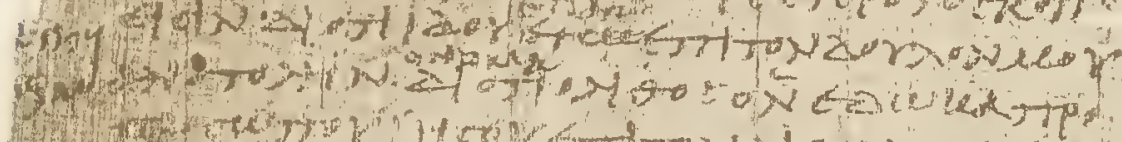

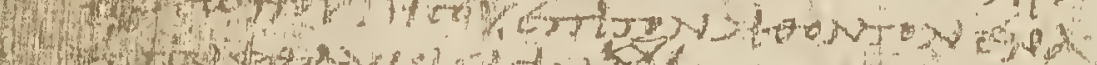
ow

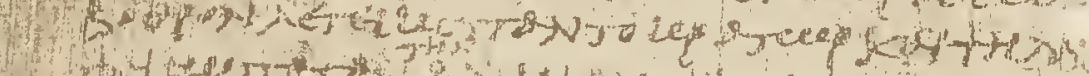

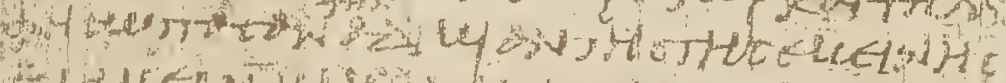
W. tow then

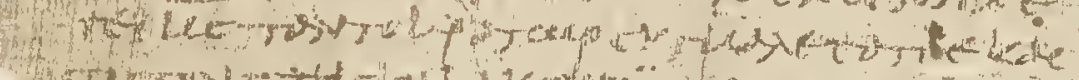

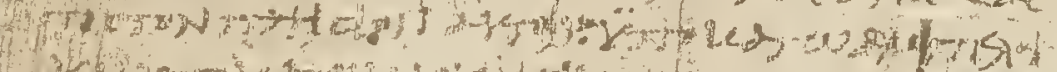

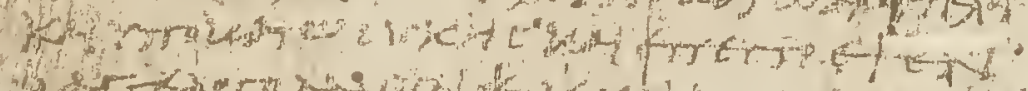

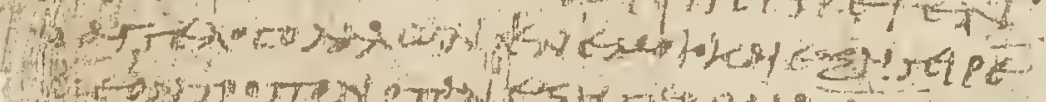
A th

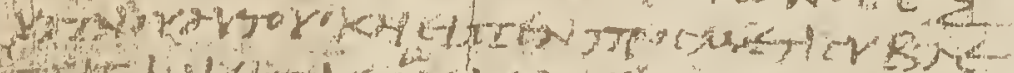

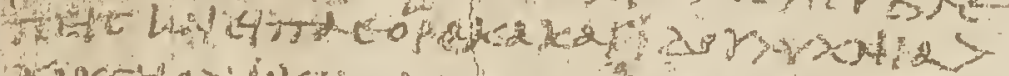

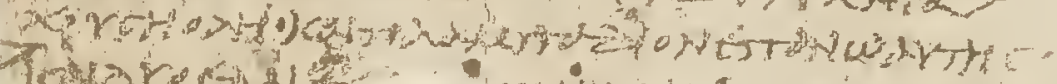

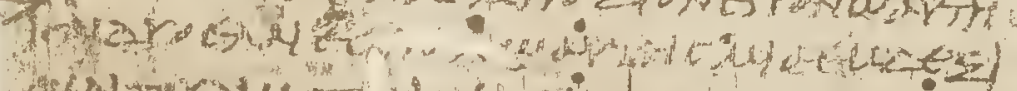

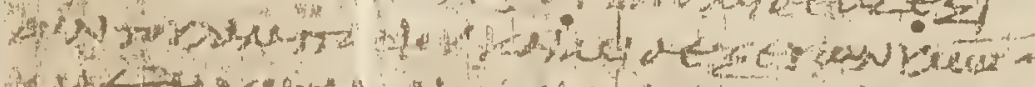

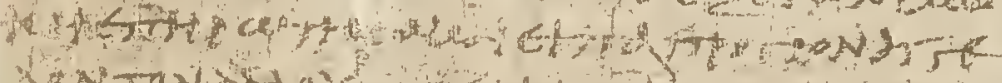

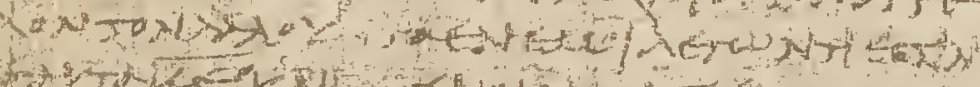

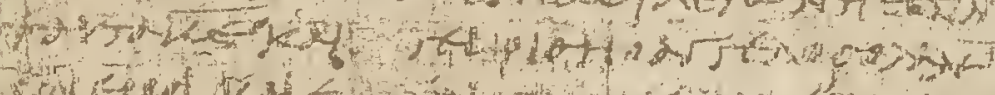

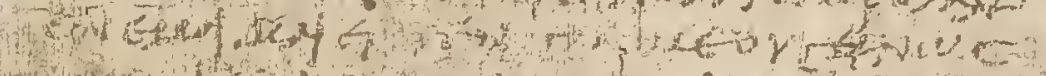

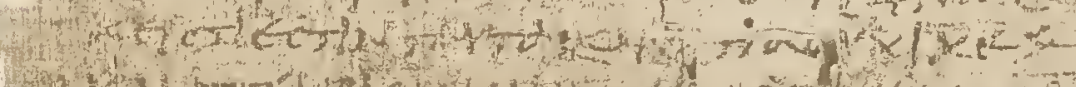
(f)

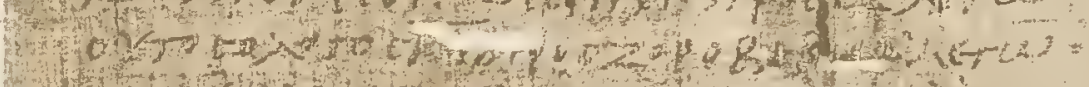

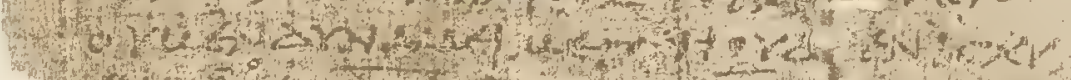

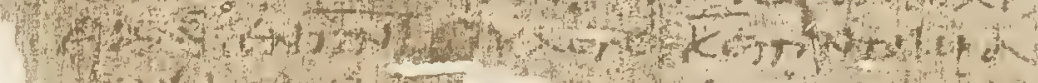

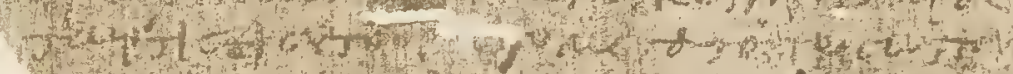

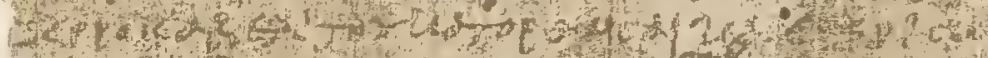

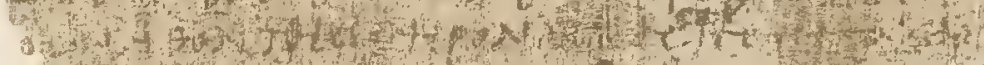

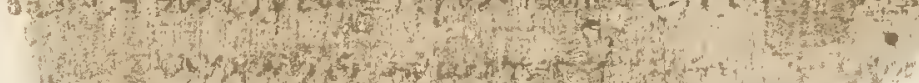

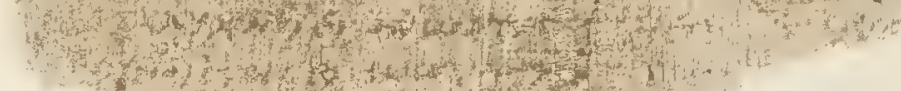

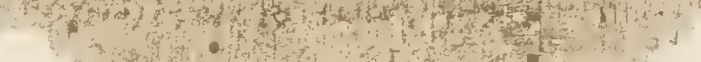
S 


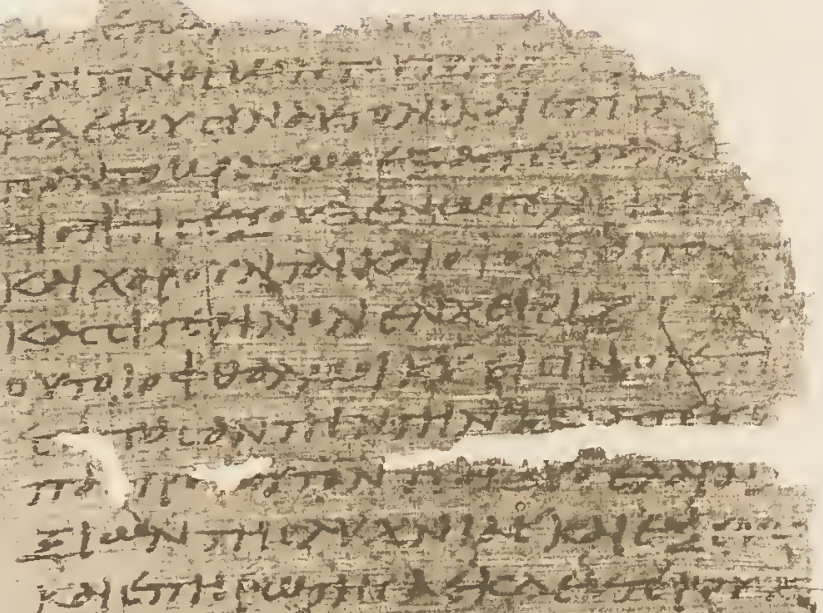

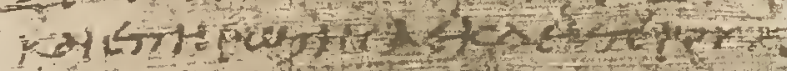

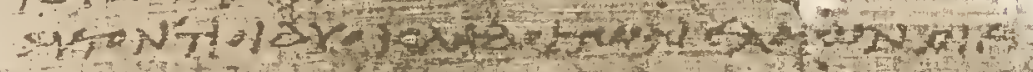

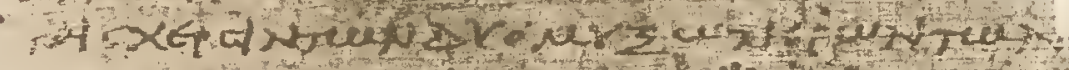

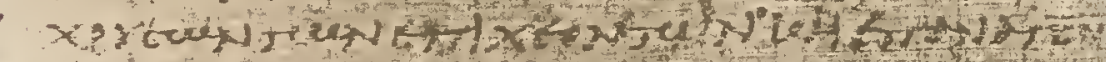

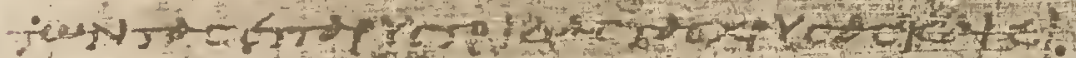

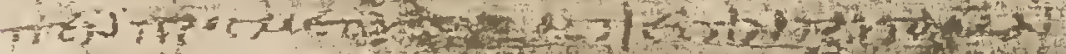

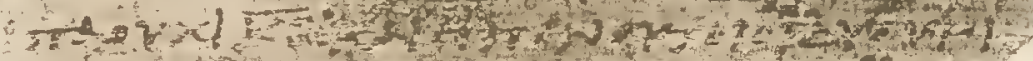

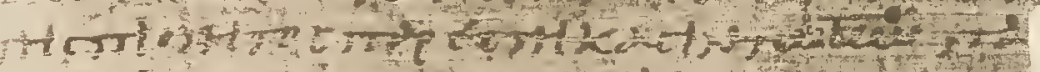

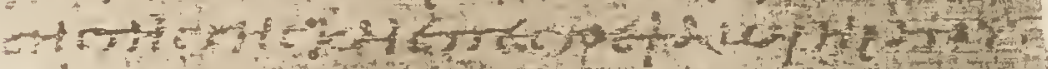

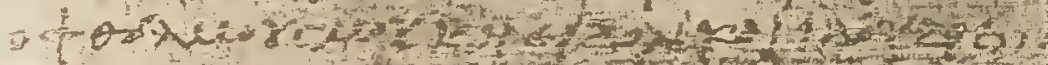

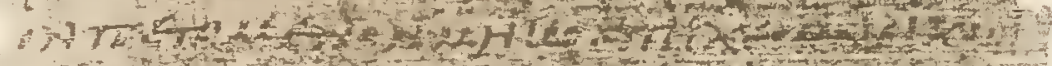

-

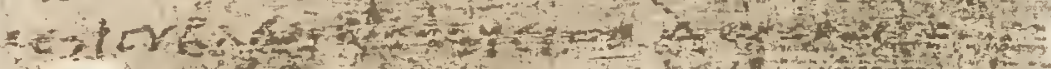

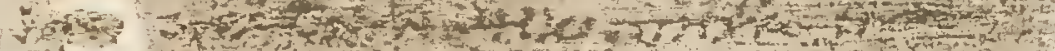

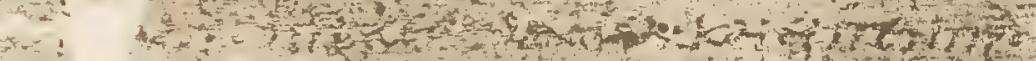

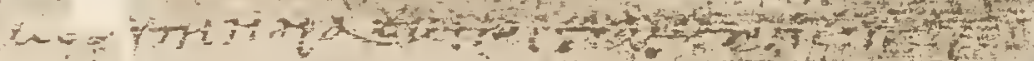

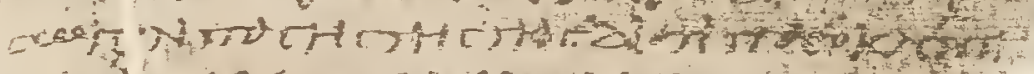

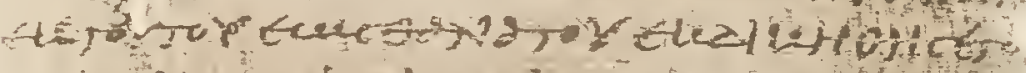

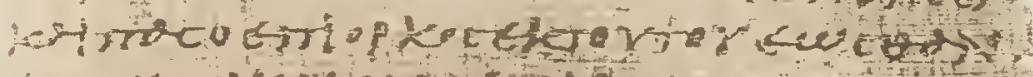
W

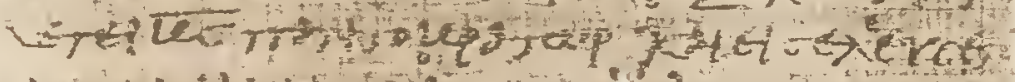

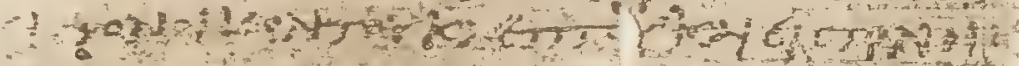
in

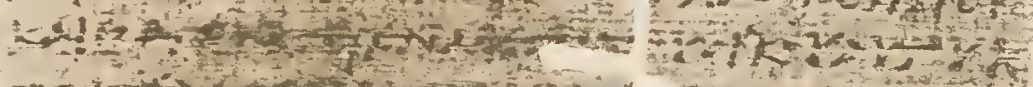

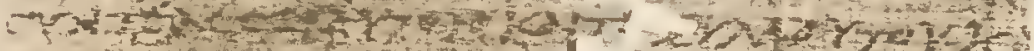

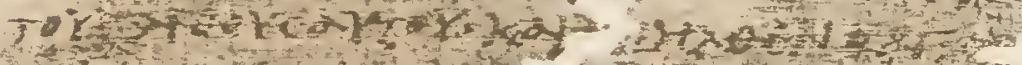

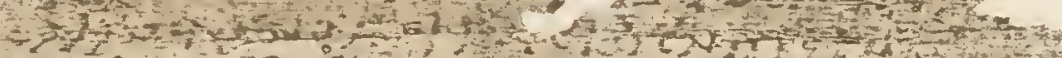

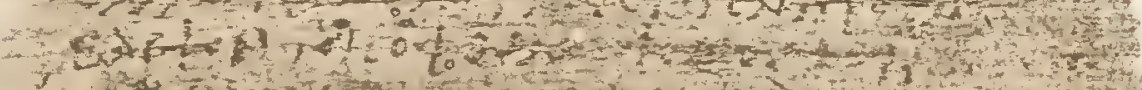
Ty

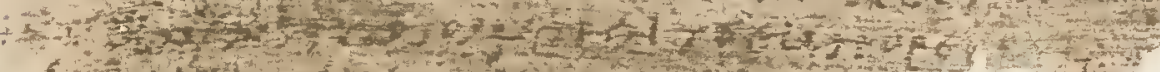

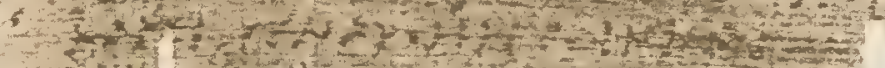

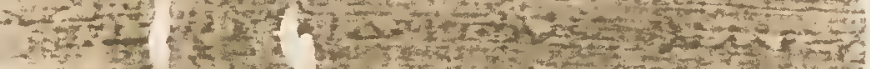

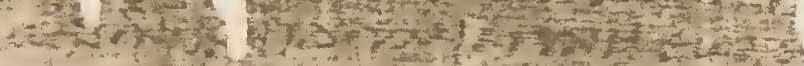

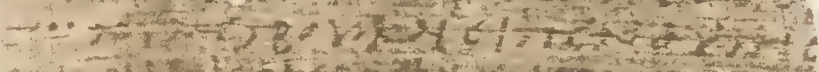

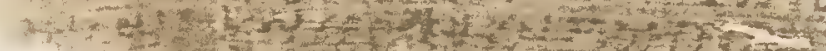

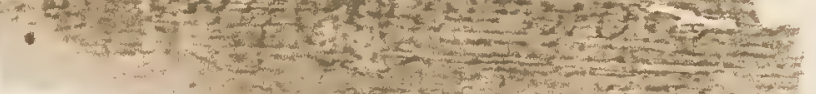

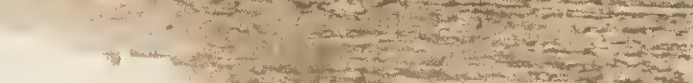

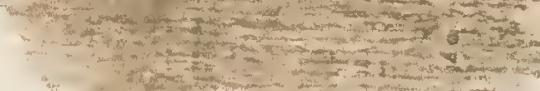

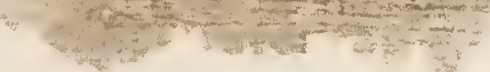




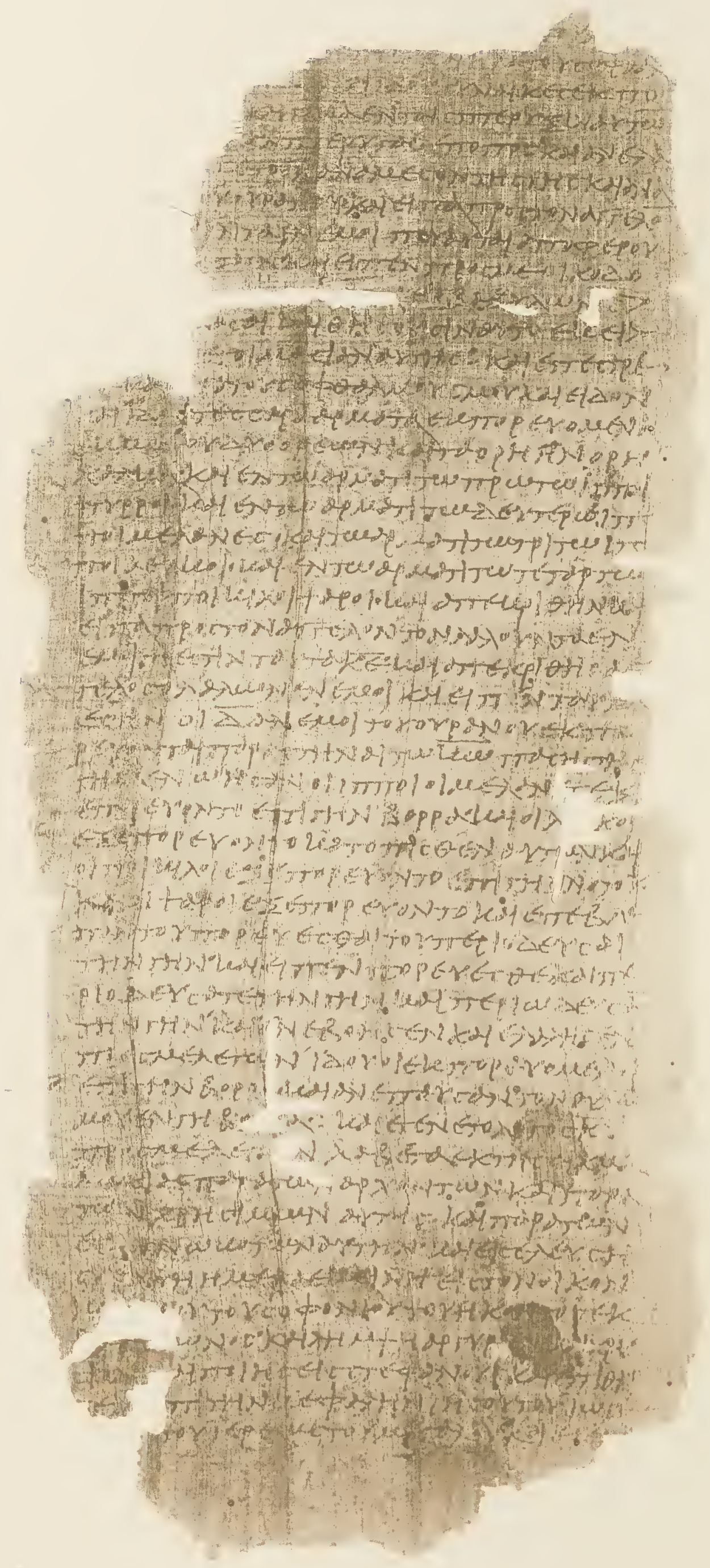

Z,ACHARIAS $v, 8$-vi, 12 


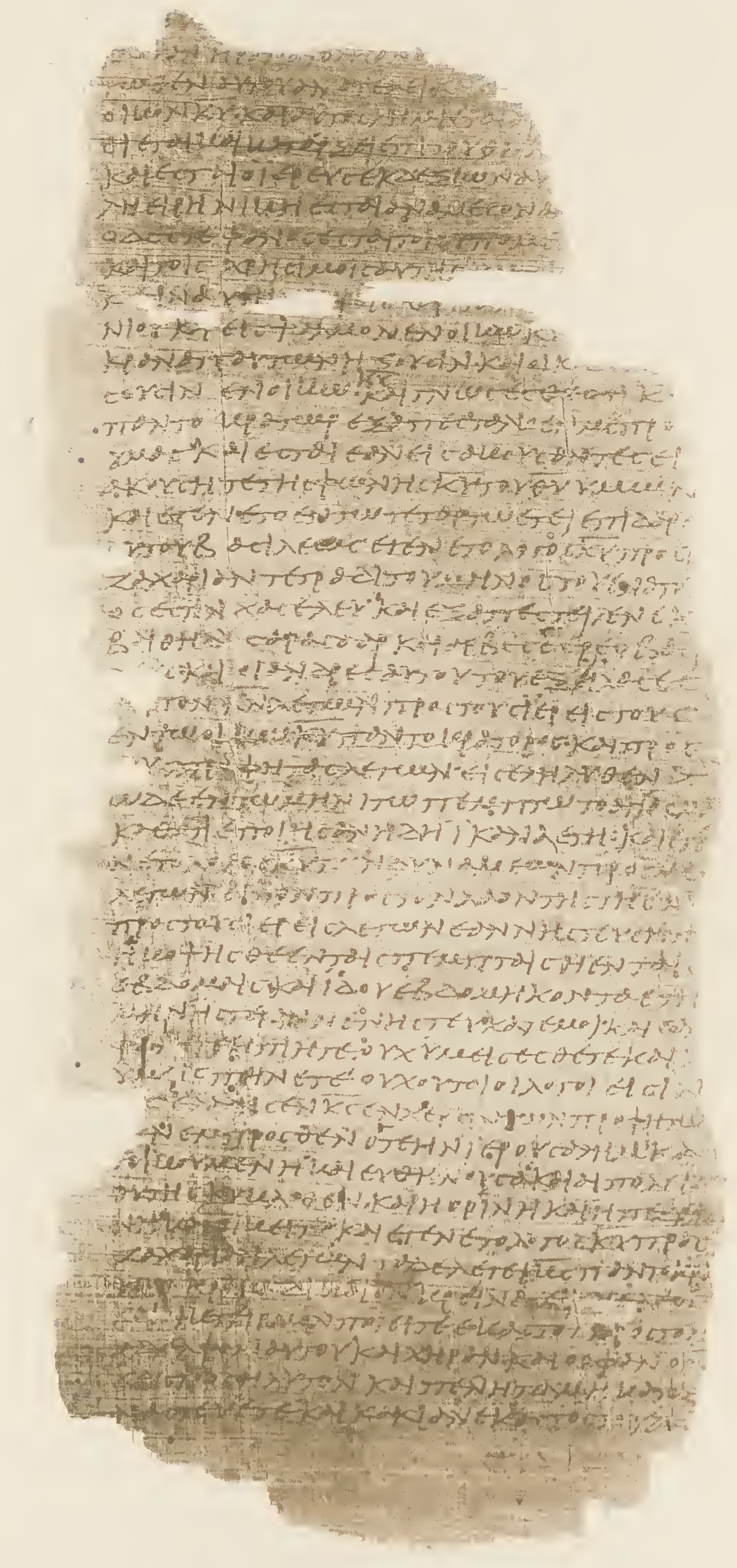




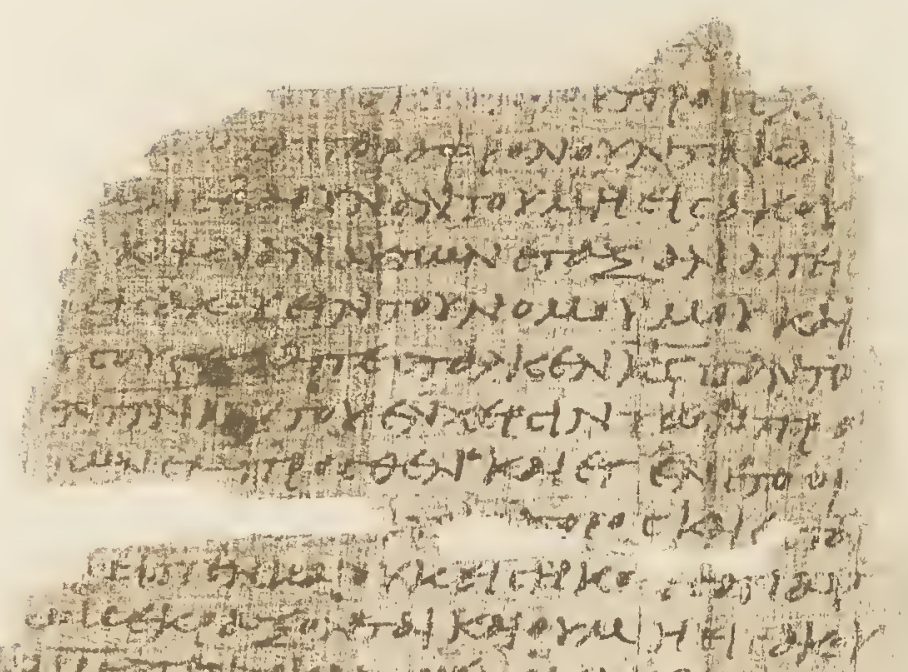

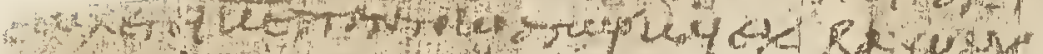
Workt

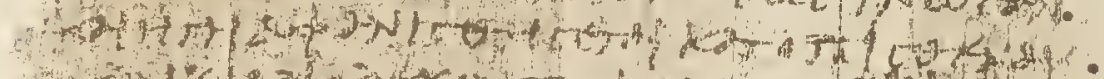

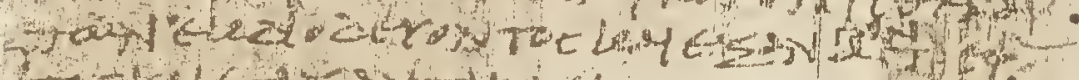

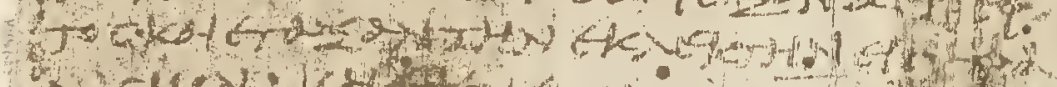

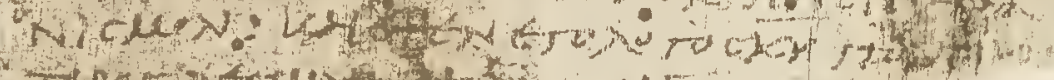

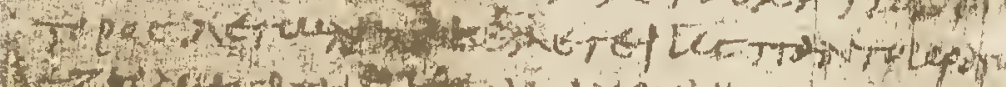

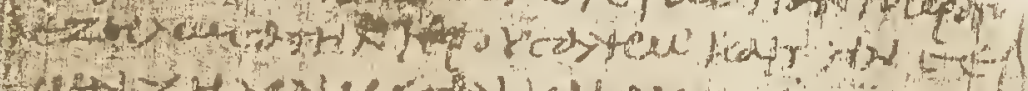

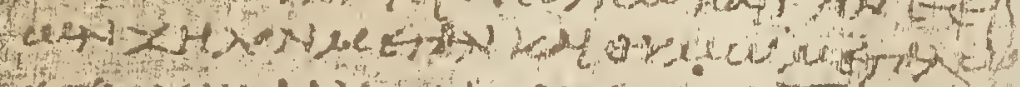

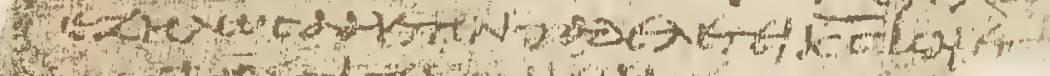

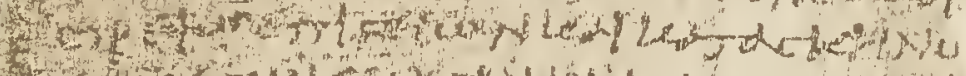

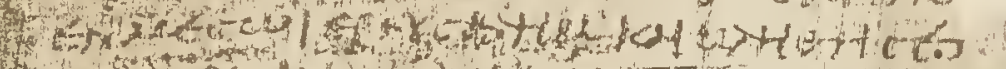

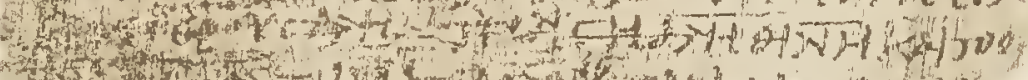

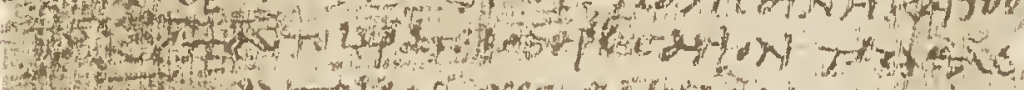

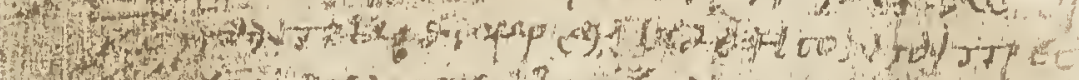

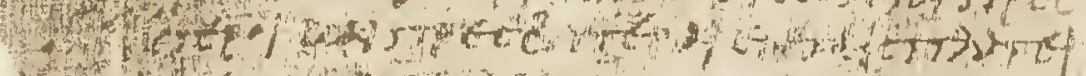

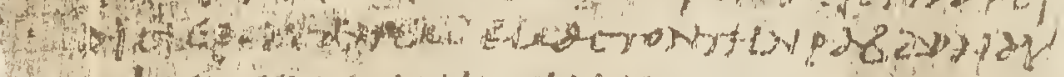

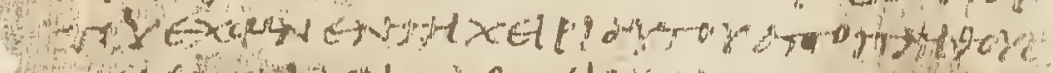

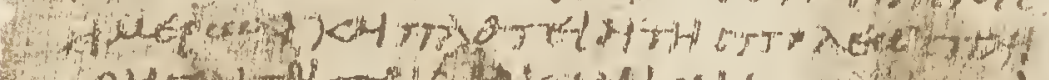
-

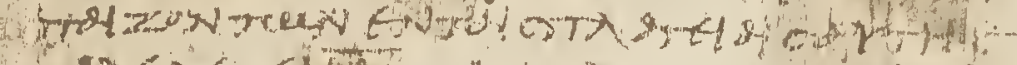

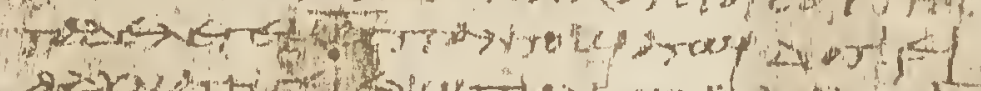

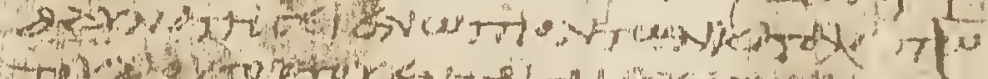

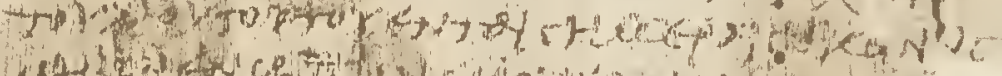

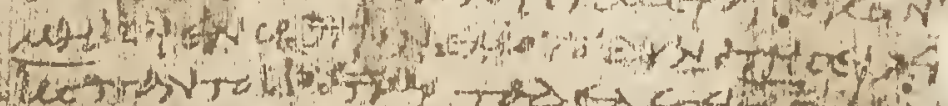

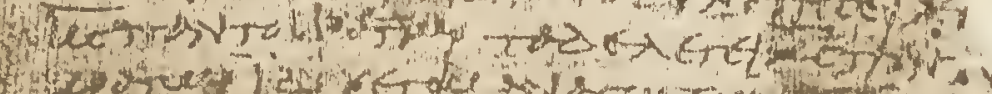

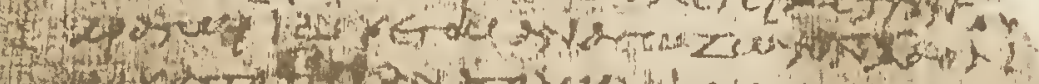

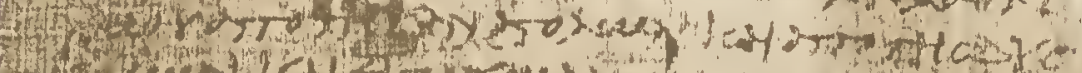

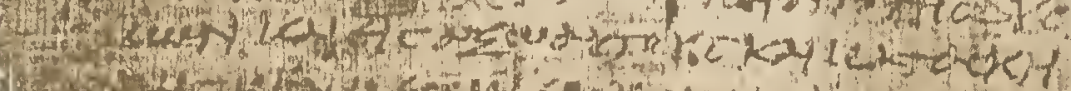

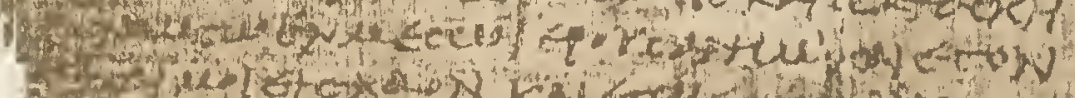

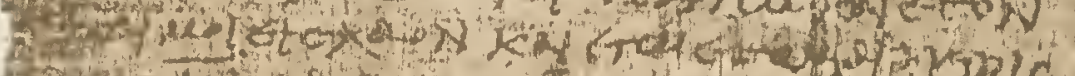

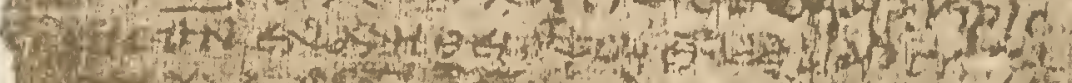

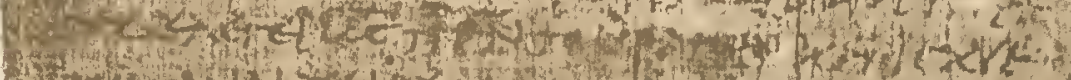

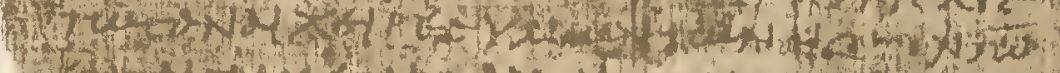

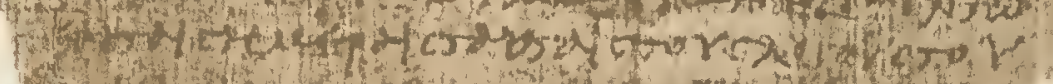

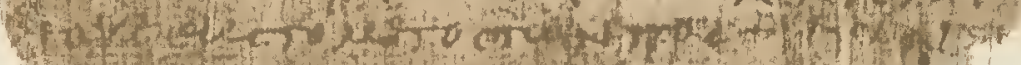

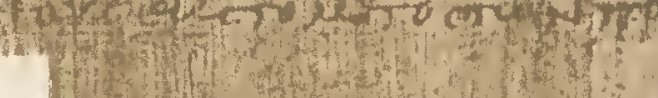

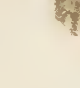
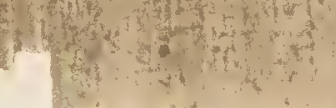


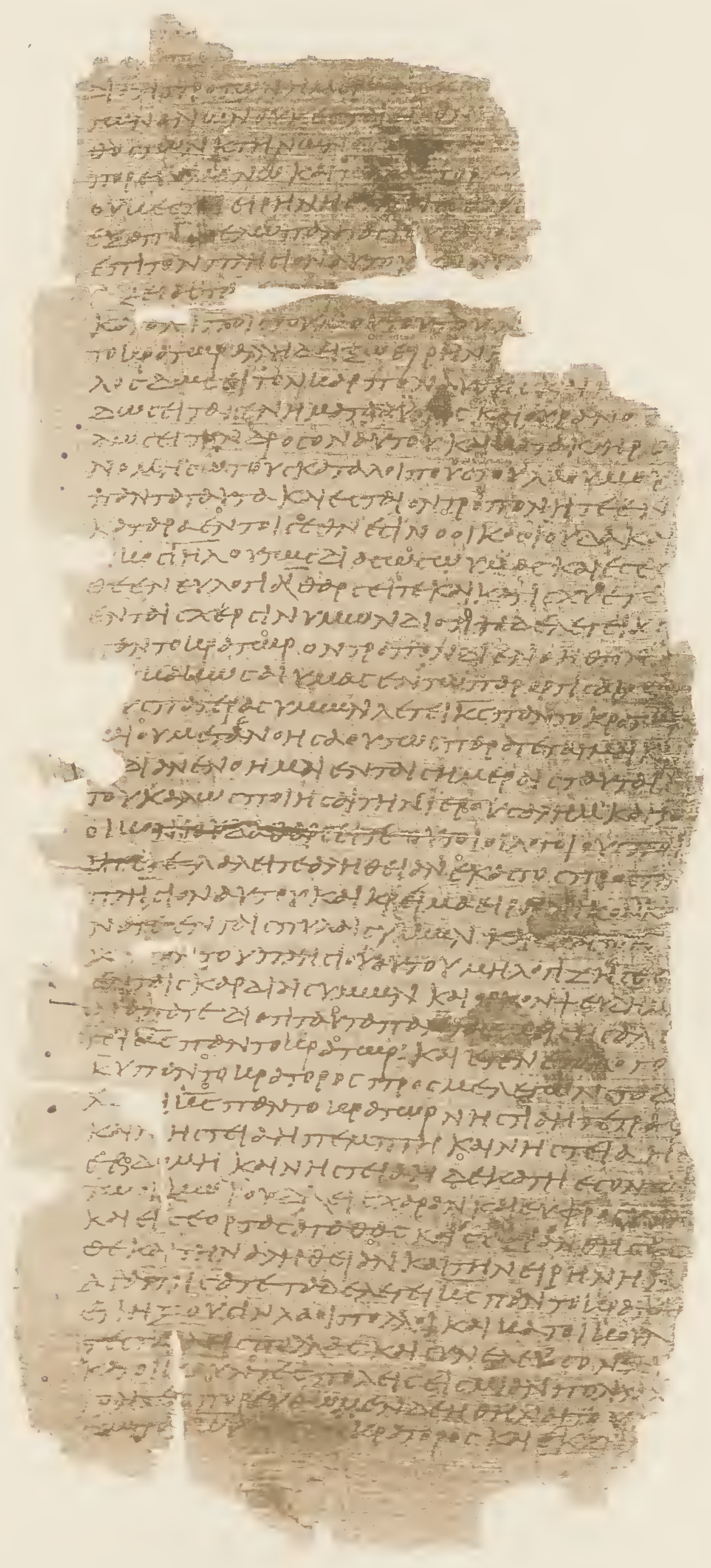

Zacharias viii, 921. 


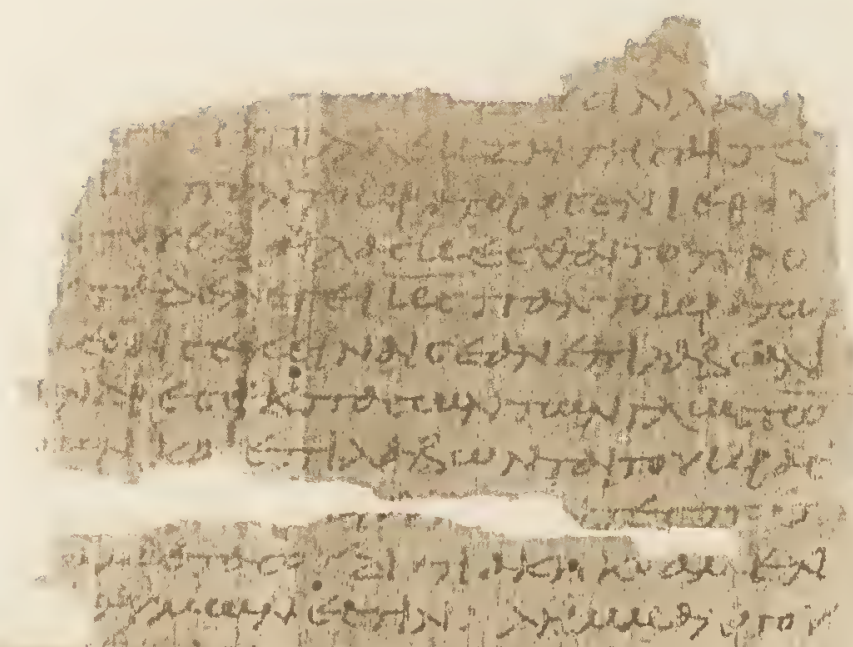

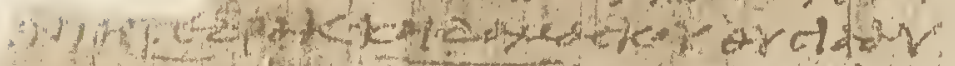

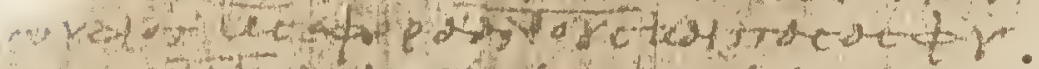

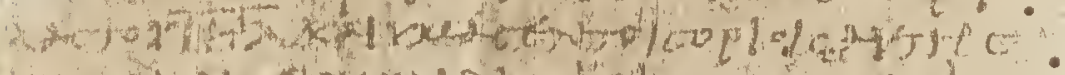

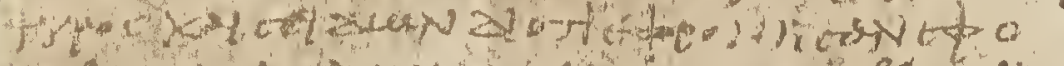

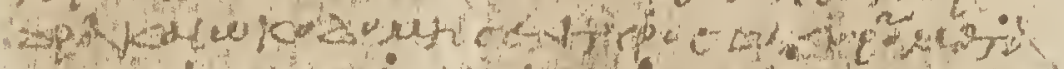

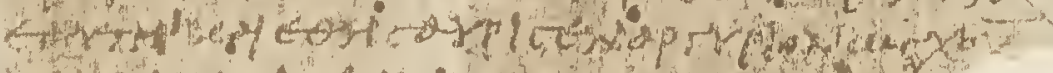

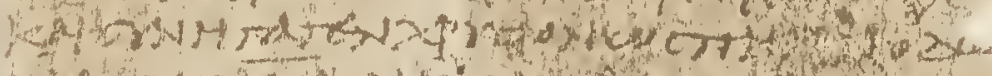

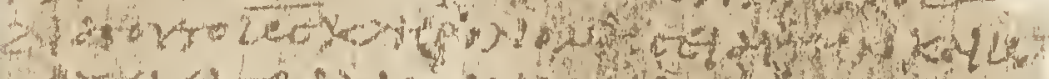

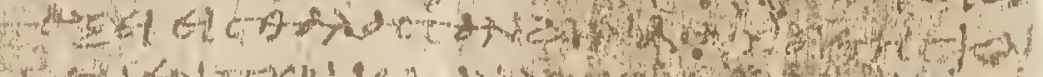

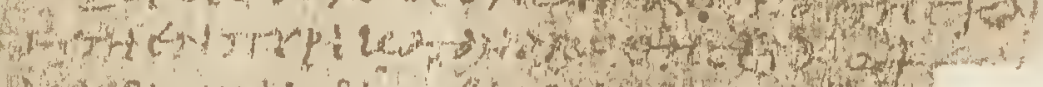

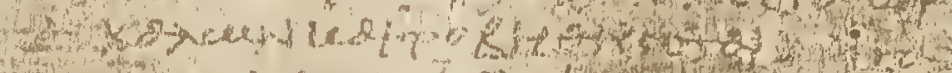

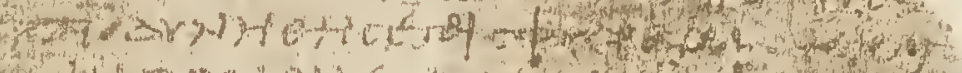

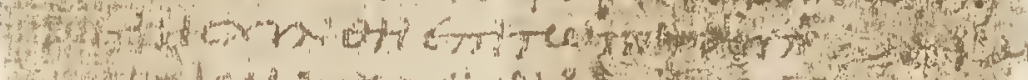

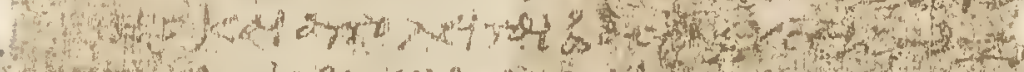

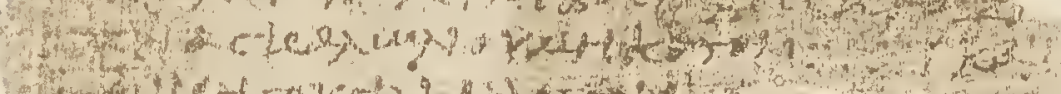
(4)

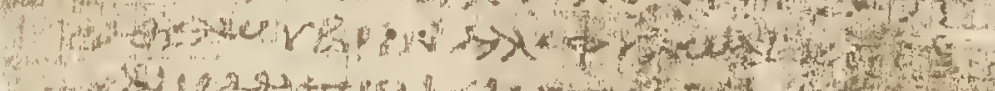

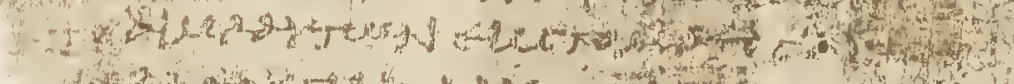

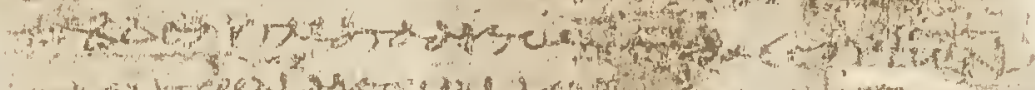

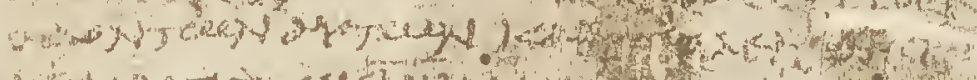

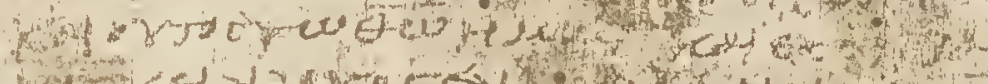

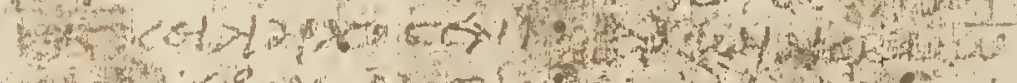

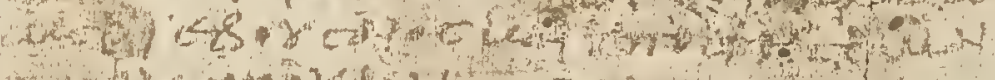

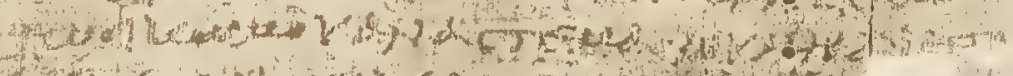

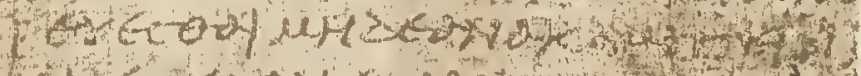

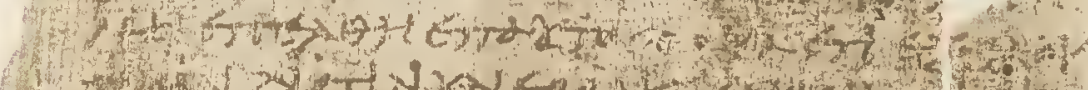

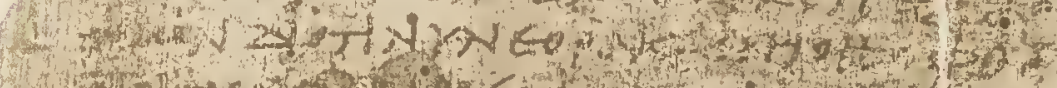

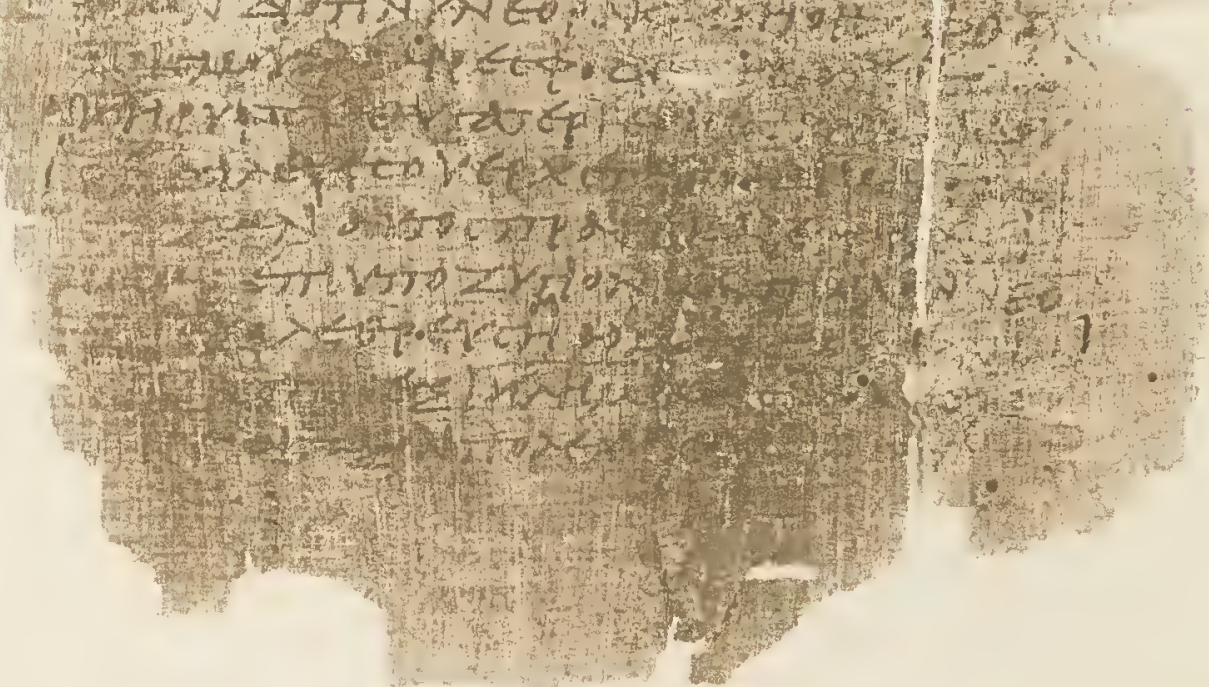




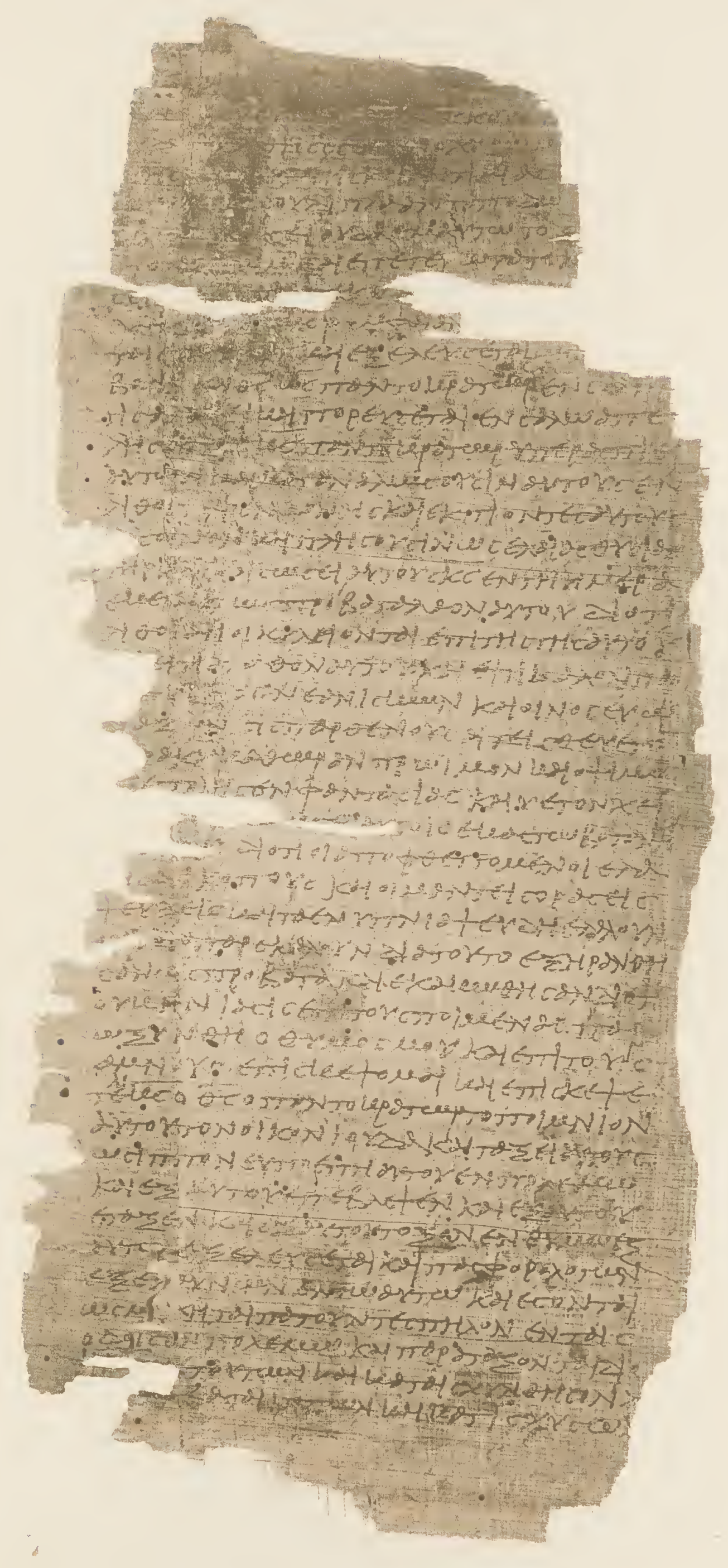

Zachartas ix, $10-\mathrm{x}, 6$. 

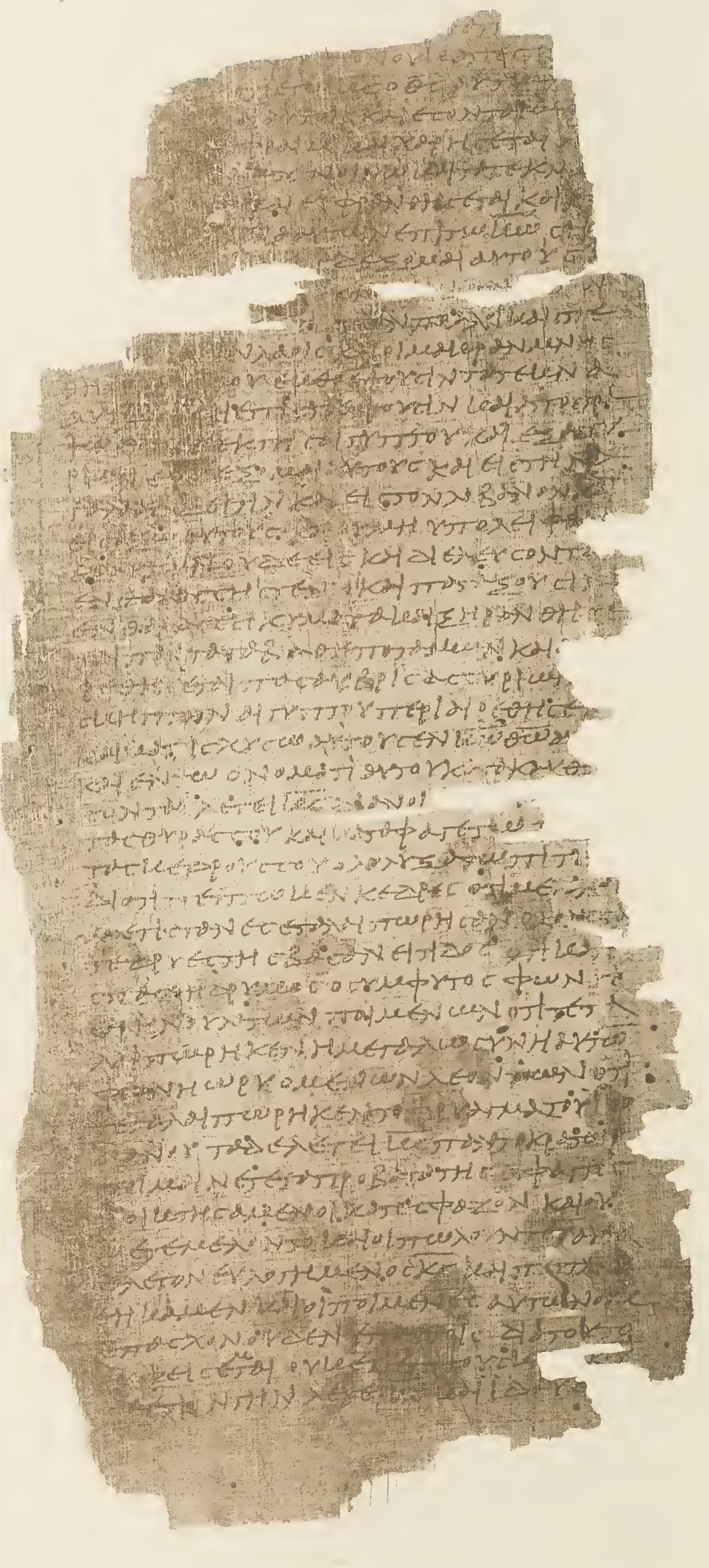

VhaCharias $\mathrm{x}, 8-\mathrm{xi}, 6$. 


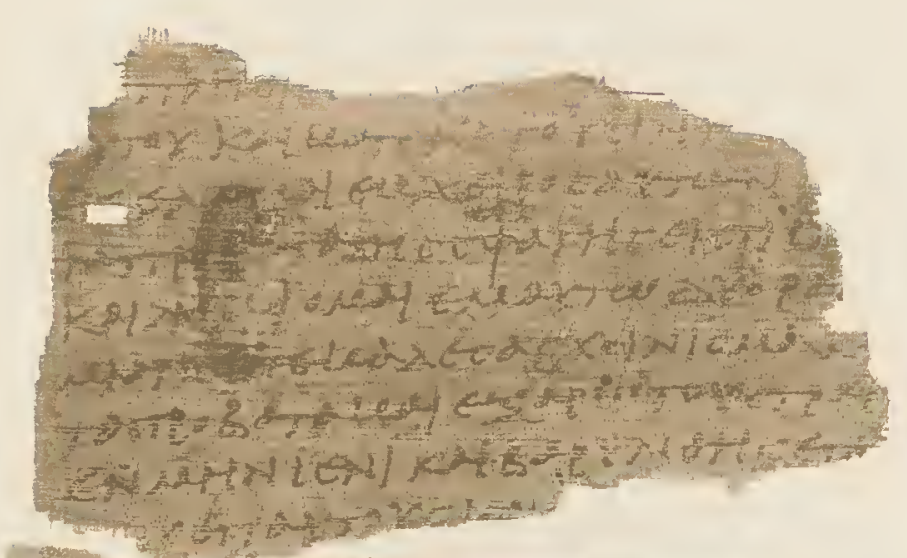

Tost ond

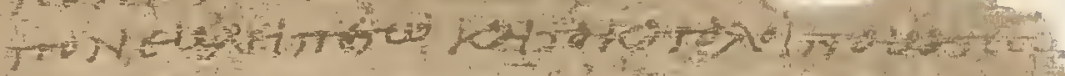

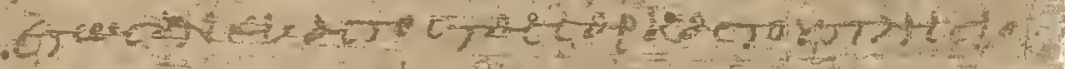

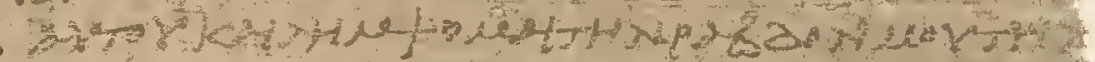

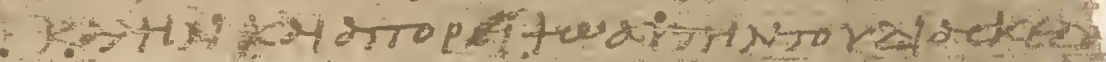

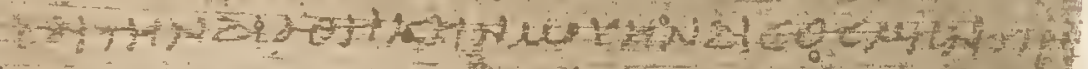

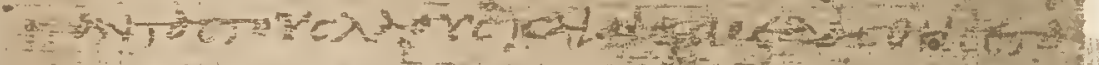

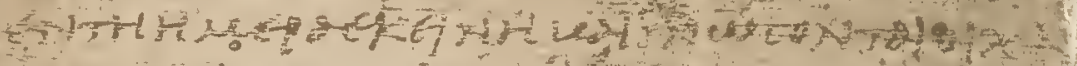

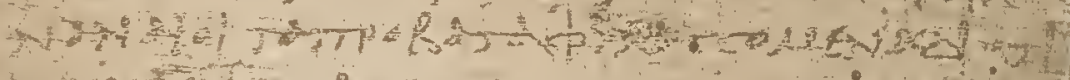

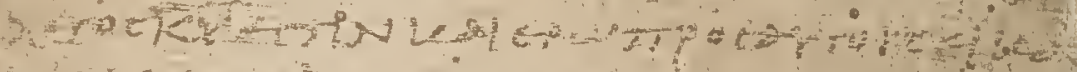

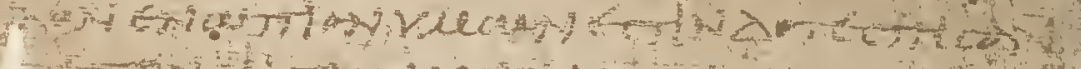

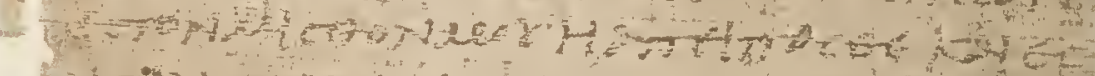

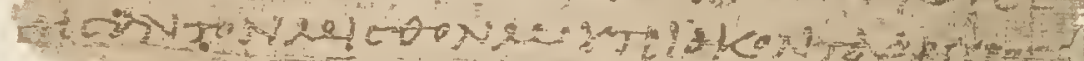

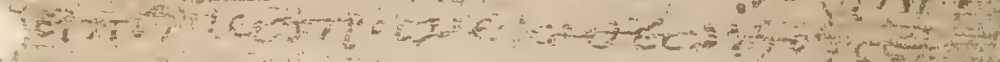

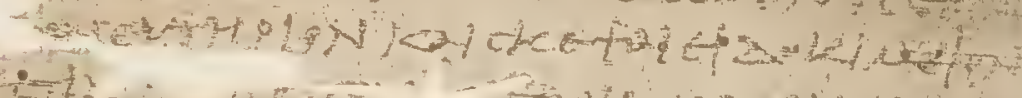

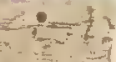

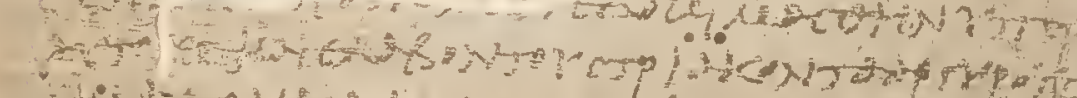
4 te

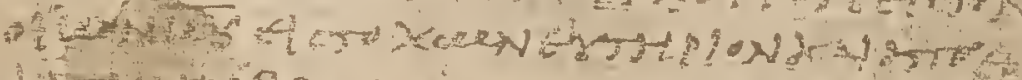

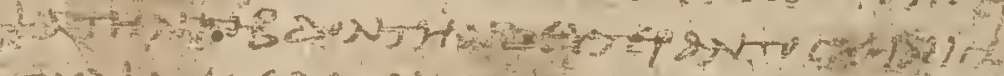

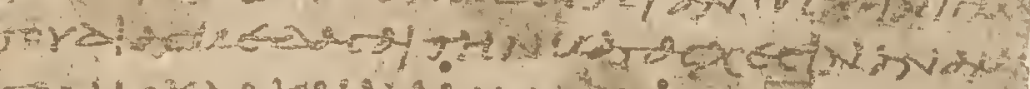

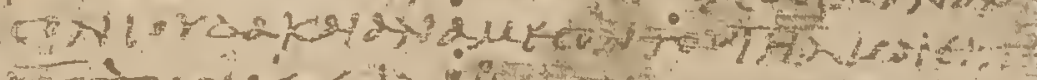

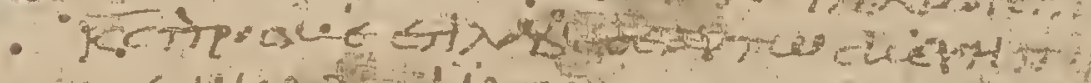

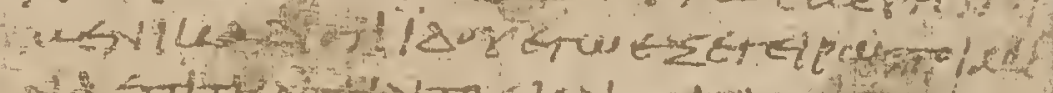

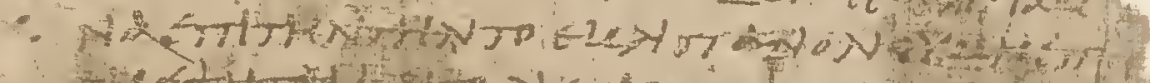

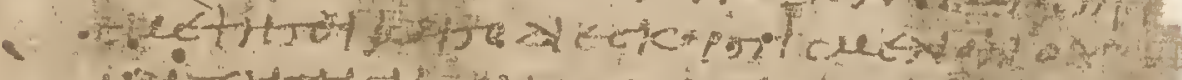

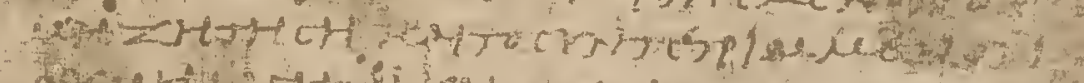

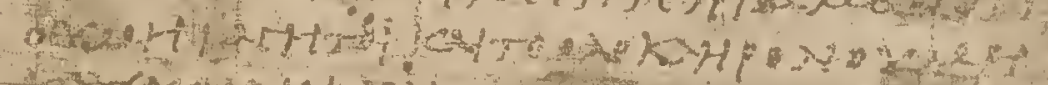

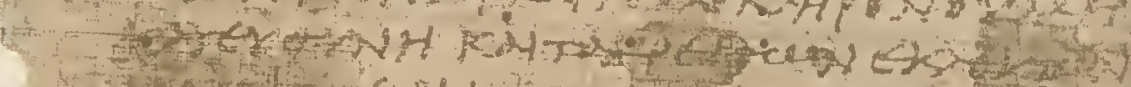

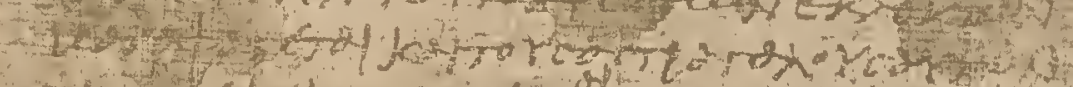

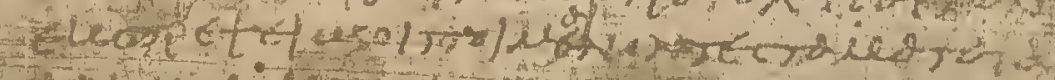

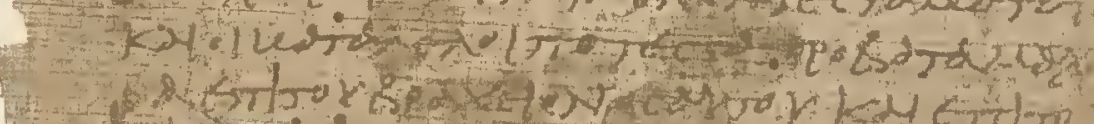

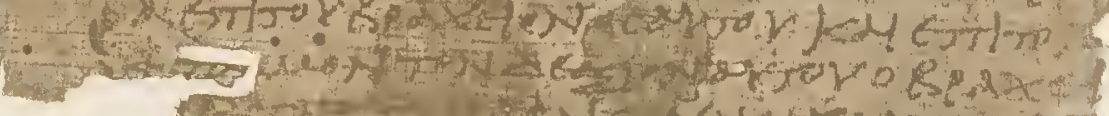

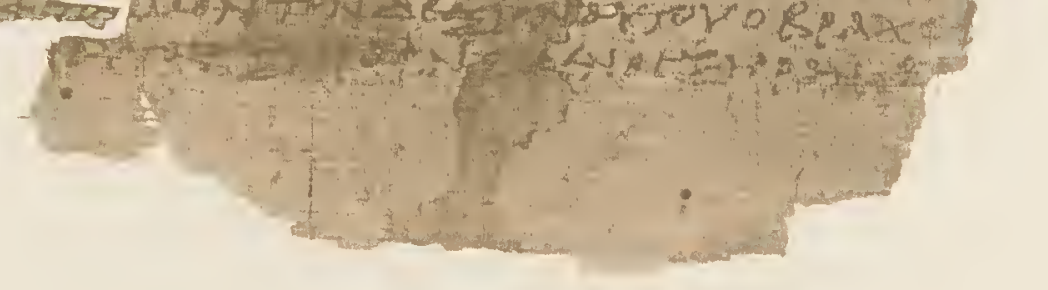



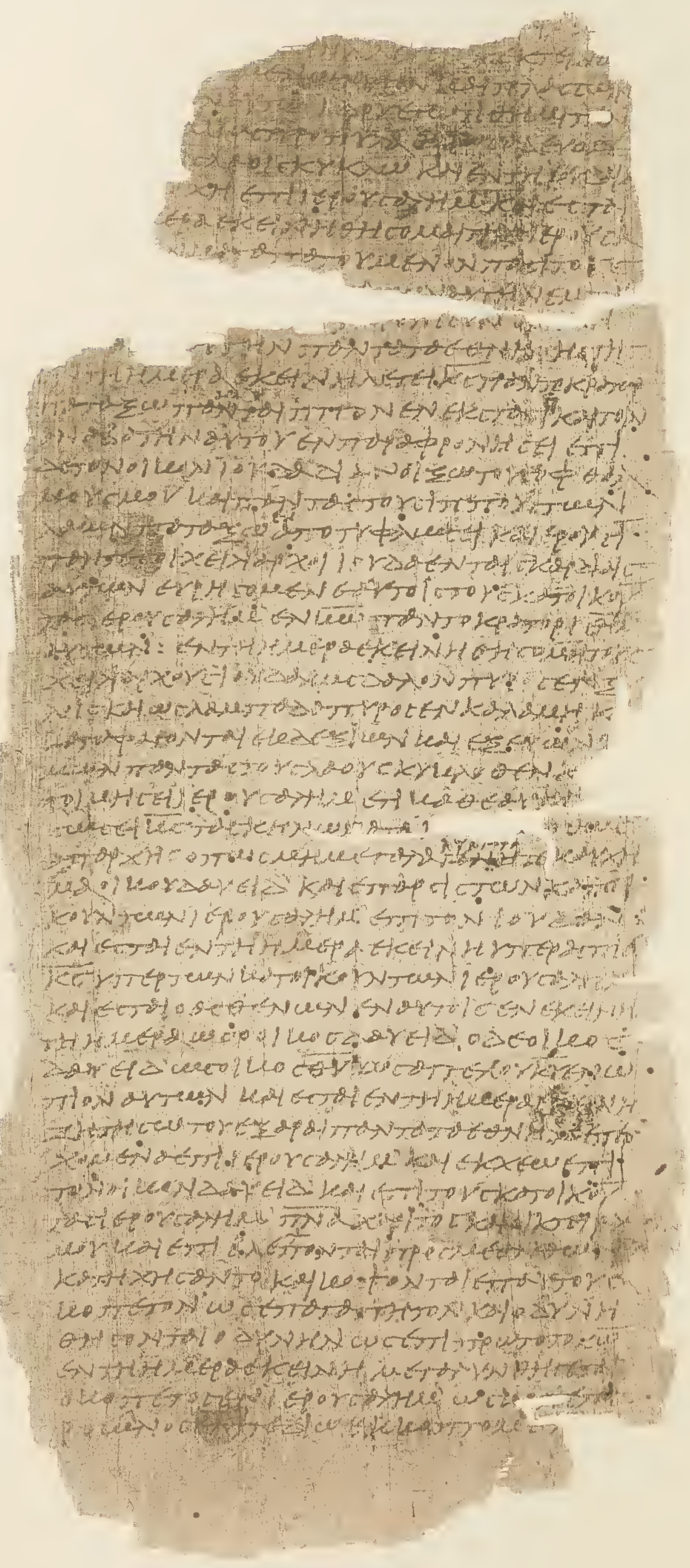

Yacularias $\mathrm{xi}, 17-\mathrm{xii}, 11$. 


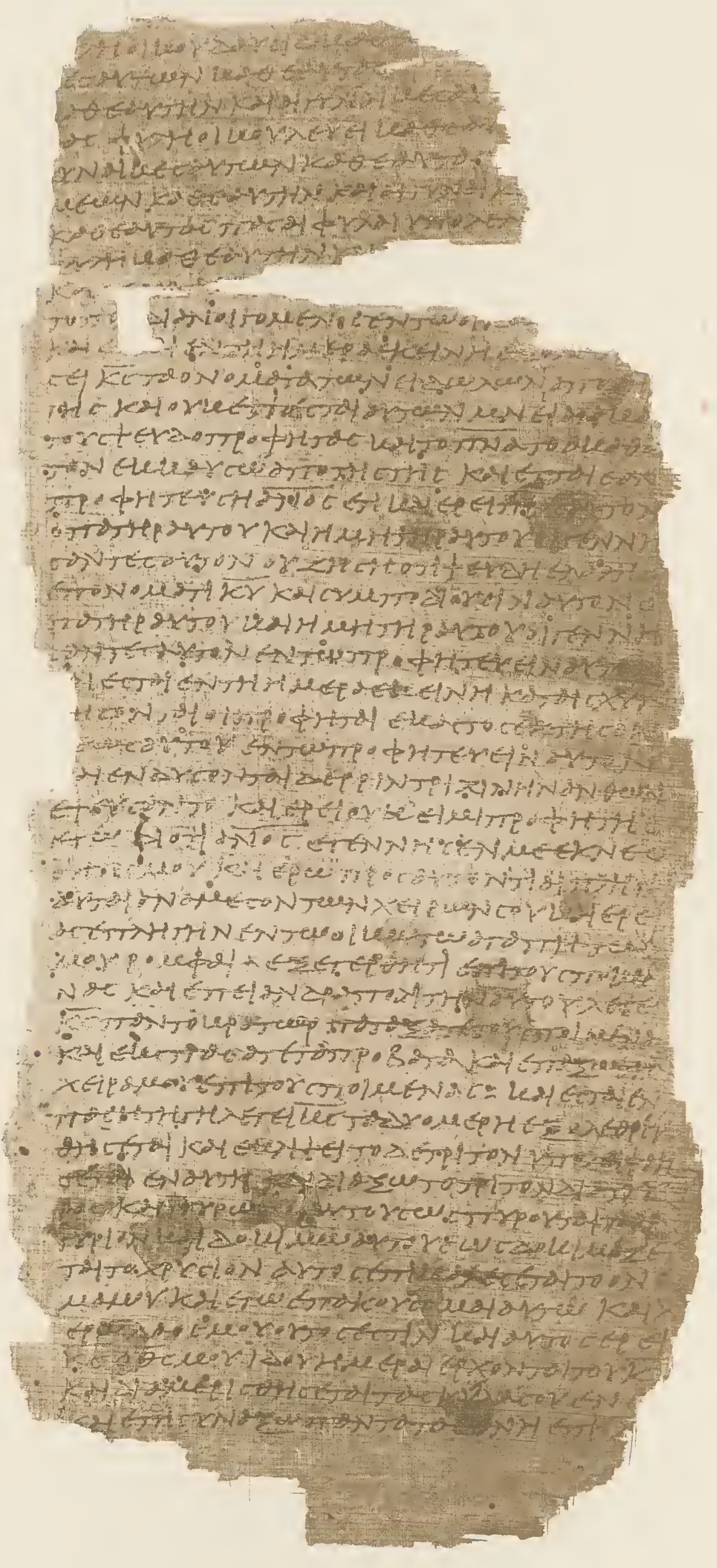

Jachartas xii, $12 \cdots$ xiy, ?. 


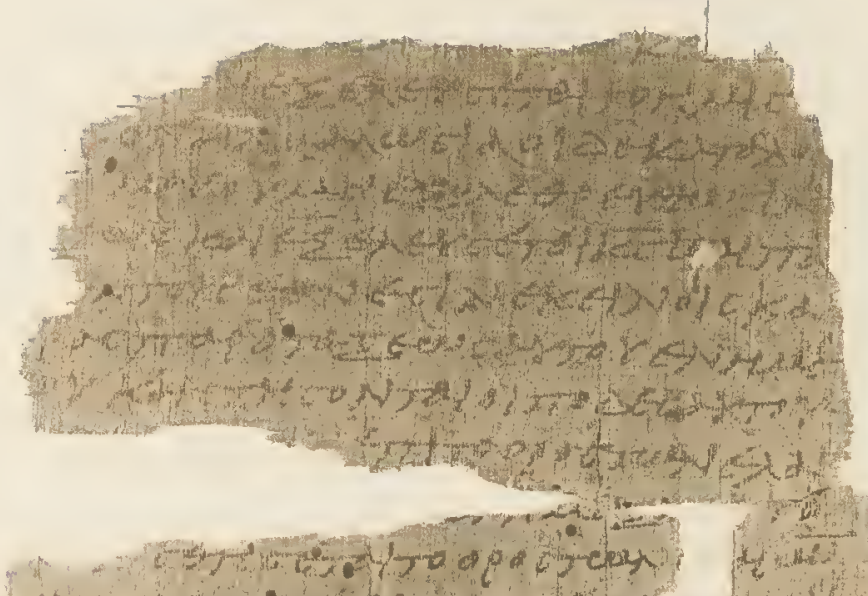

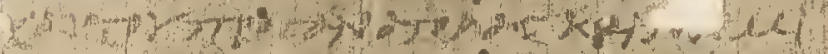

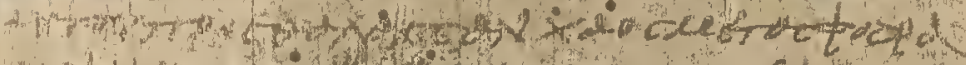

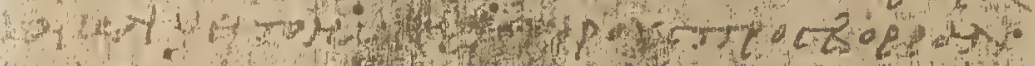

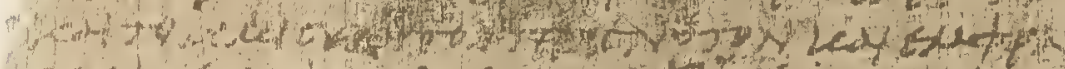

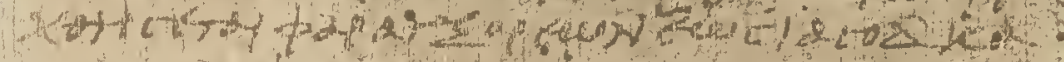

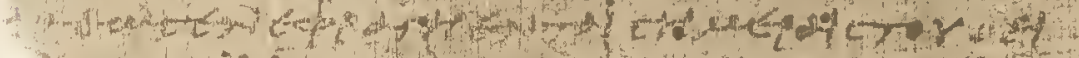

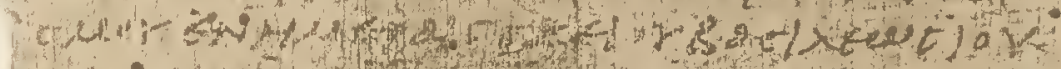

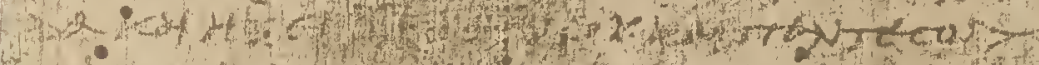

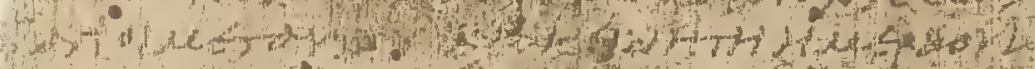

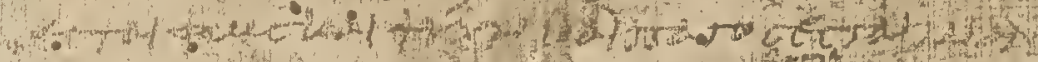

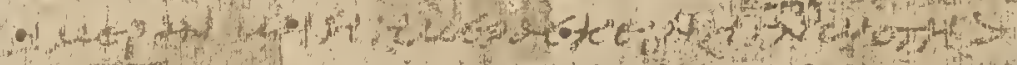

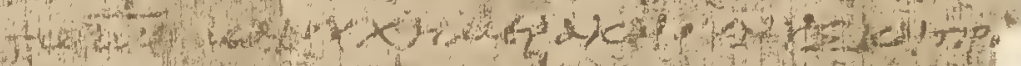

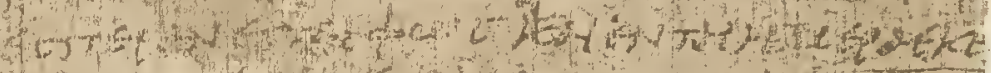

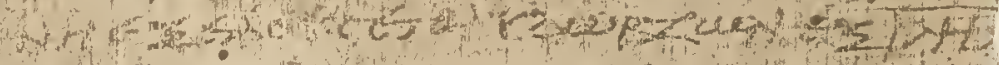

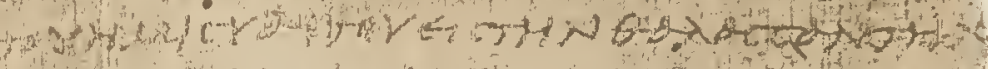

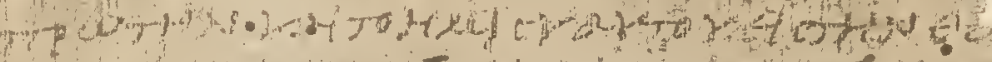

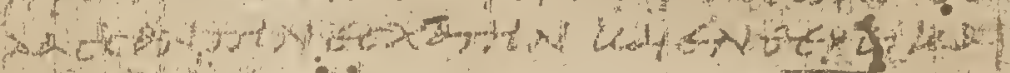

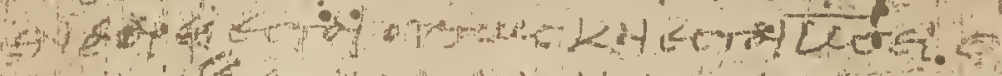

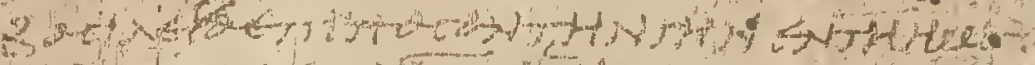

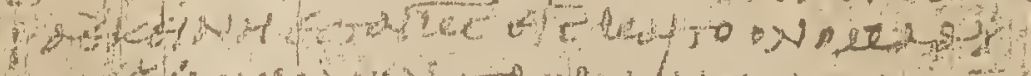

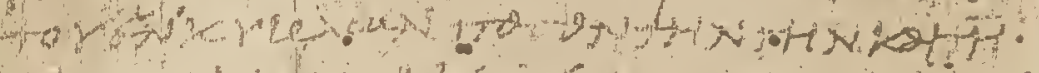

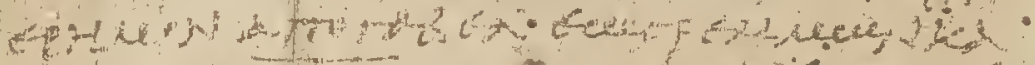
mply of

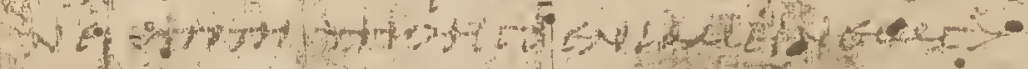

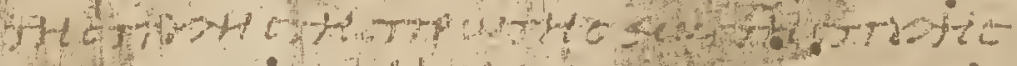

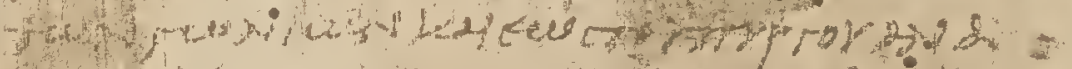

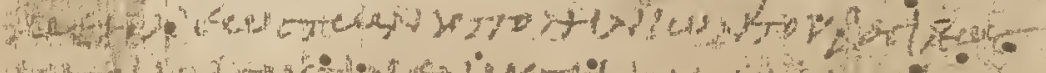

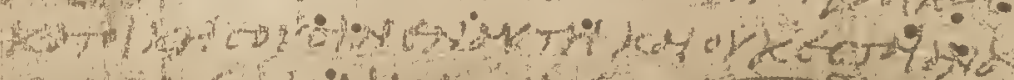

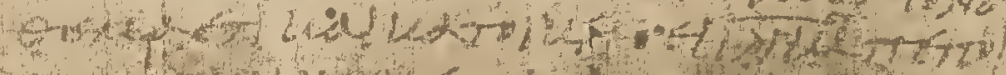

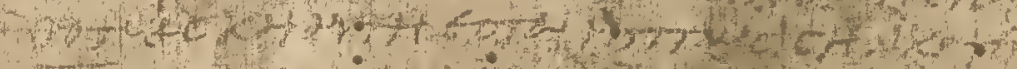

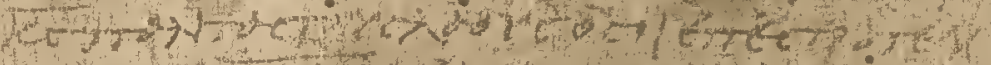

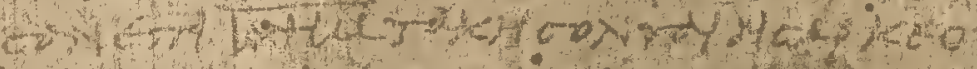

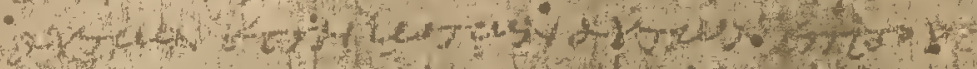

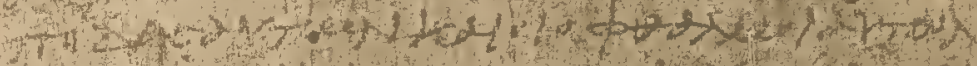

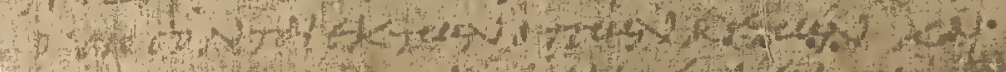

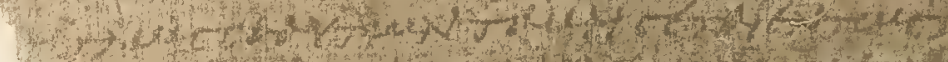

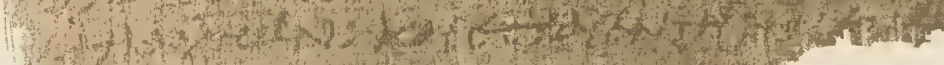
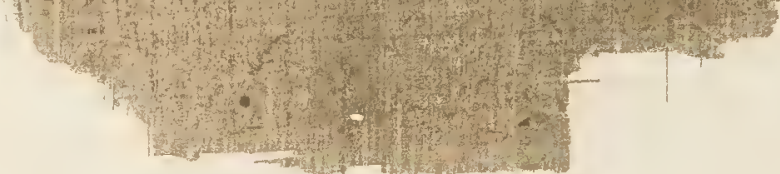


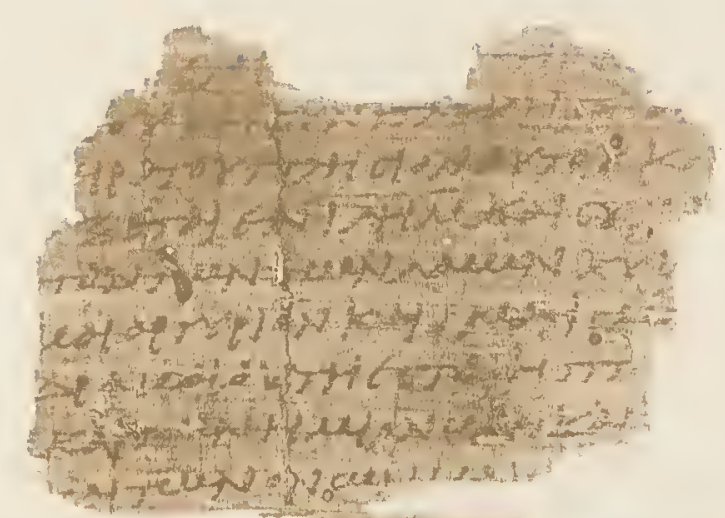

$$
\text { NGepta }
$$

an

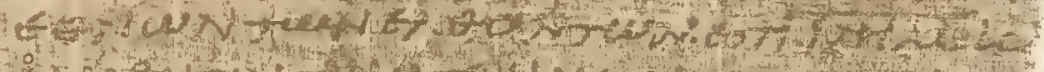

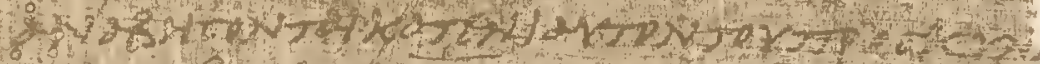

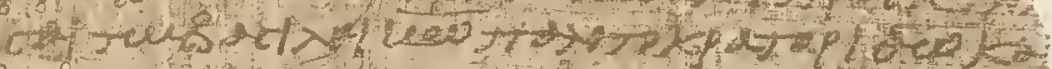

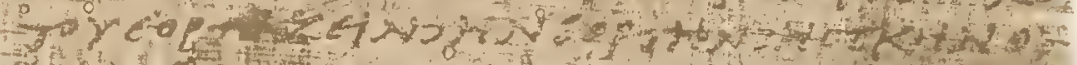

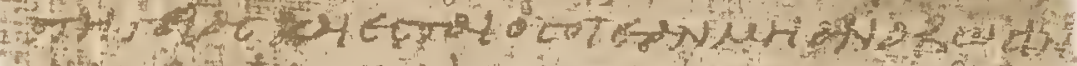

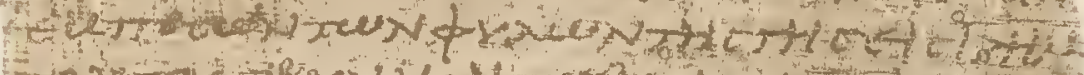

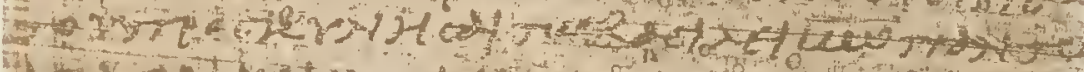

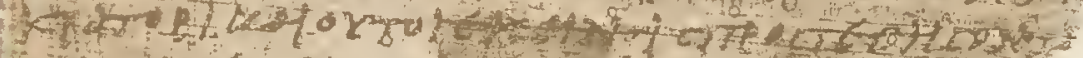

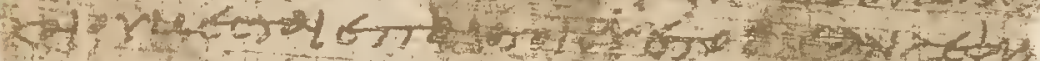

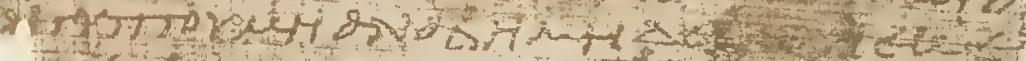

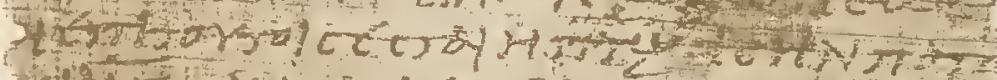

1 (t)

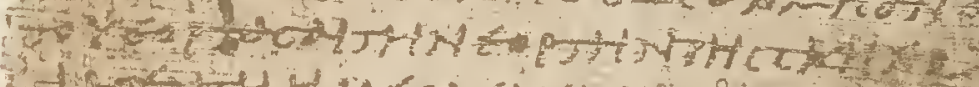

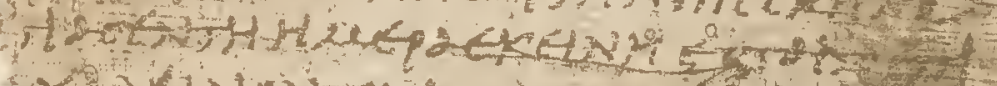

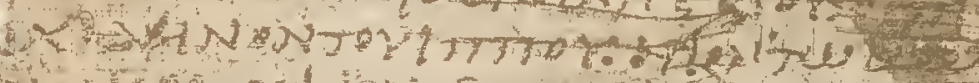

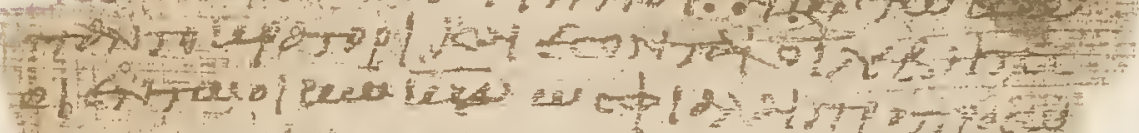

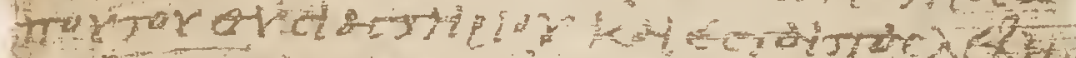
Anthin on

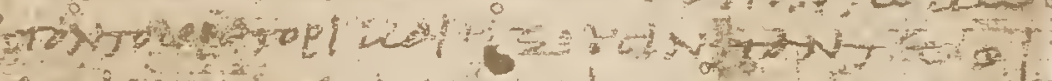

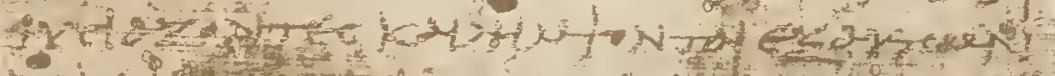

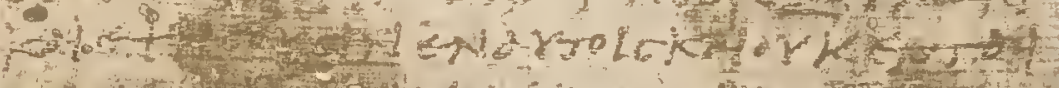

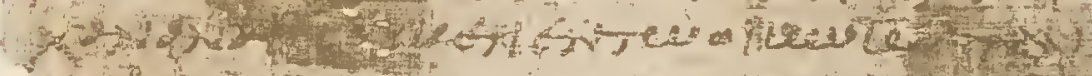

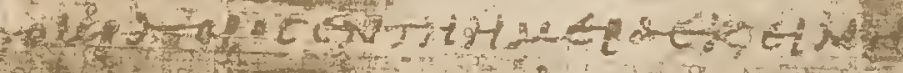

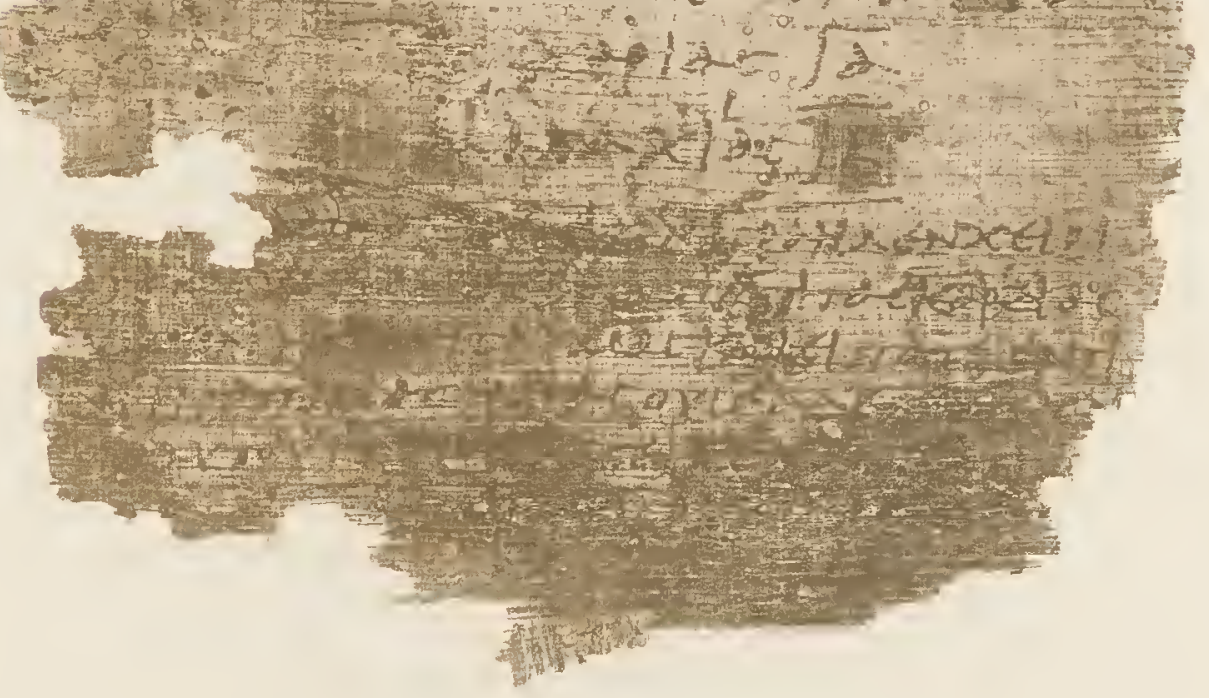




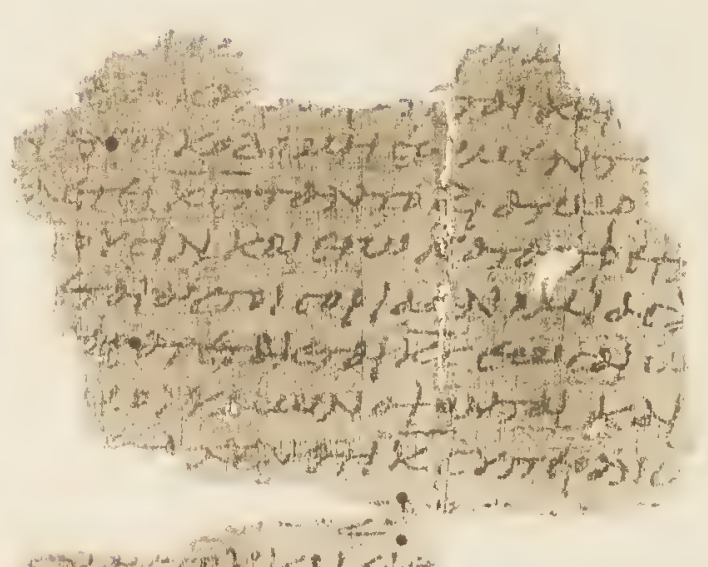

Q ?

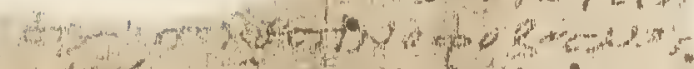

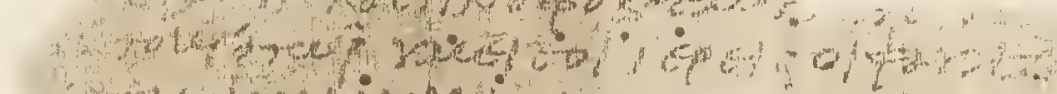

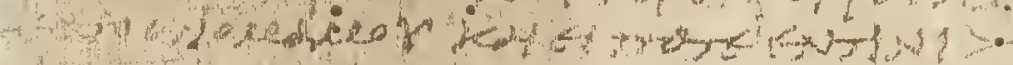

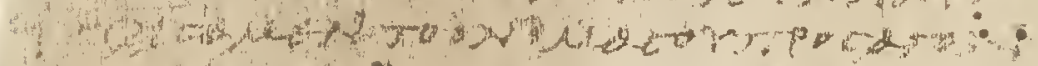

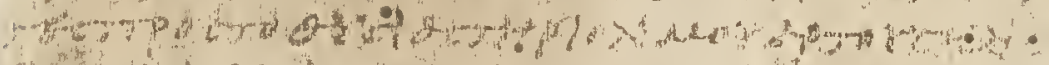

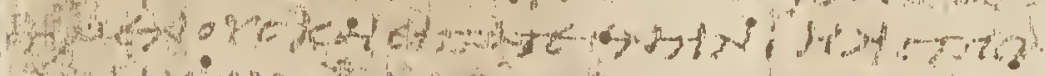

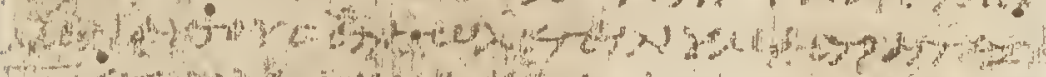

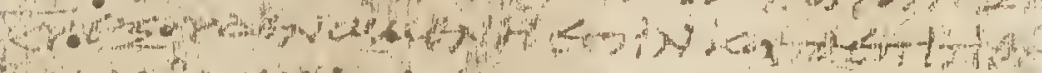

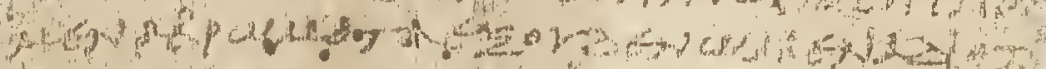

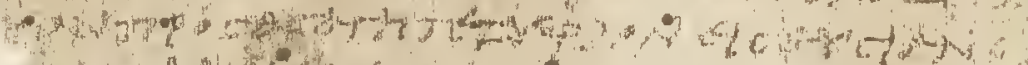

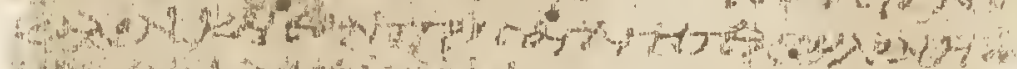

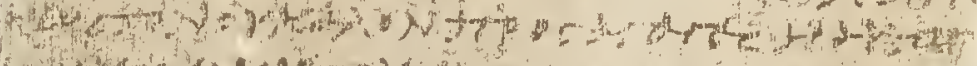

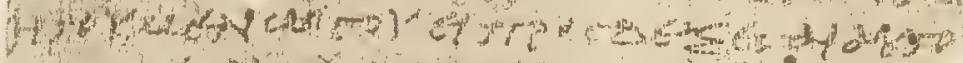

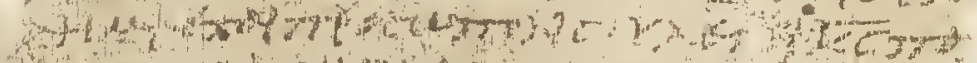

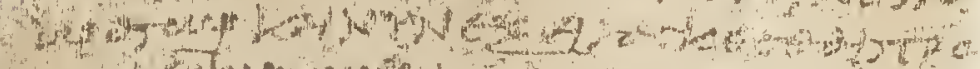

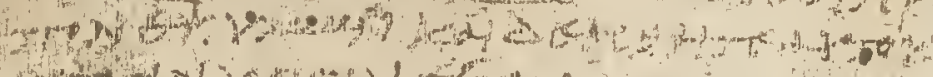

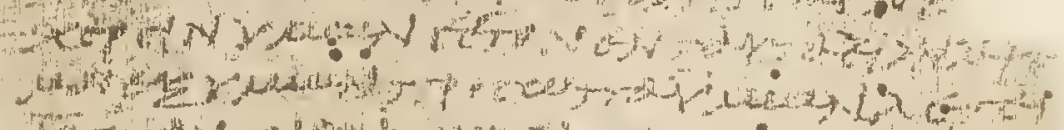

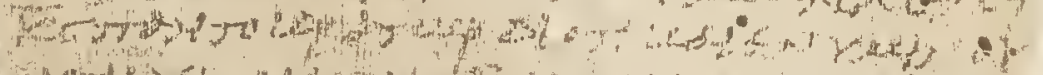

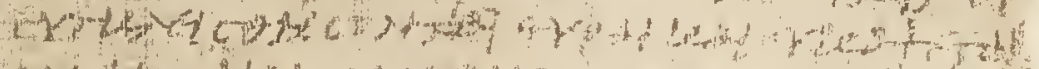

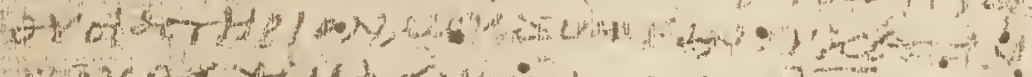

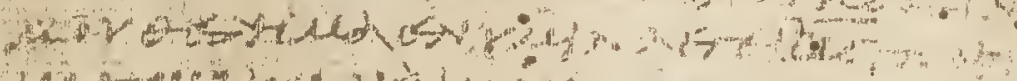

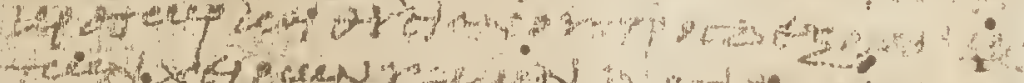

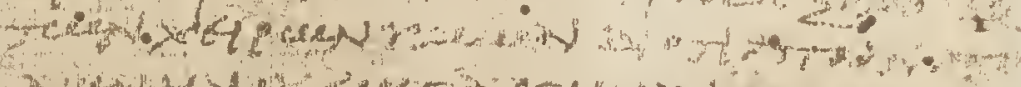

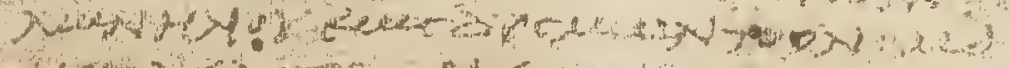

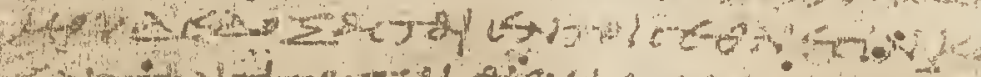

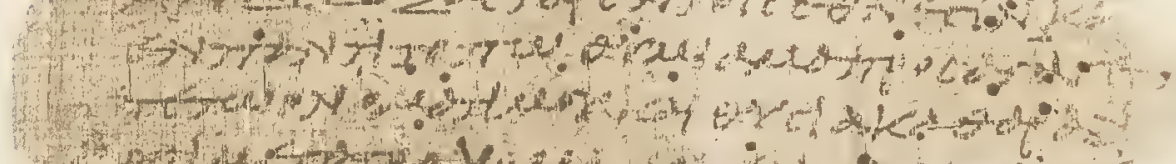

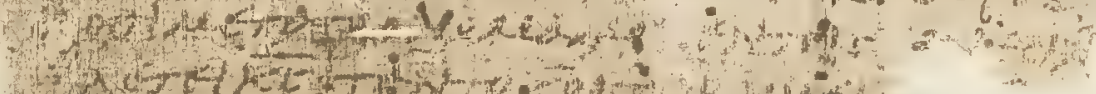

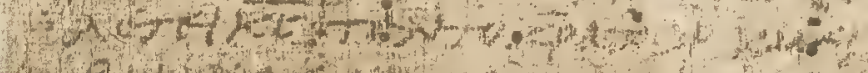

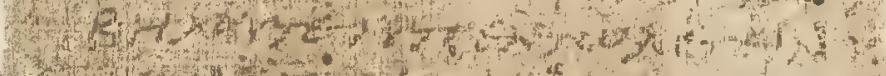

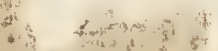

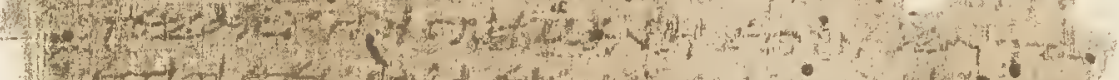

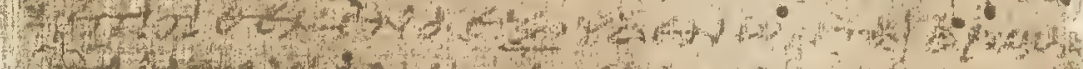

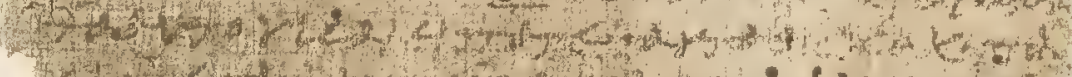

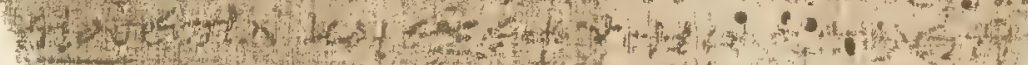

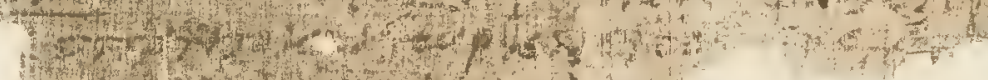

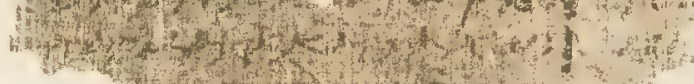

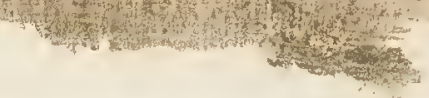




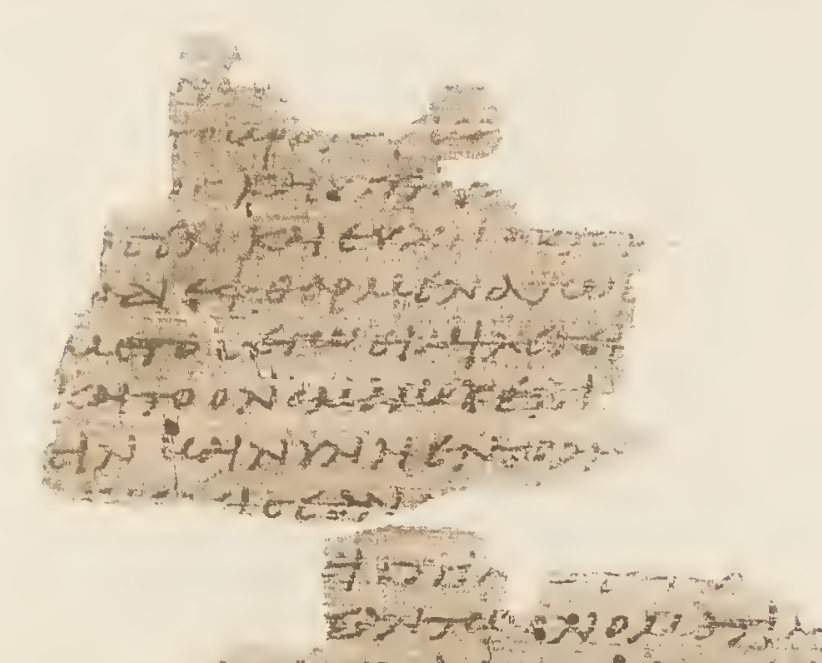

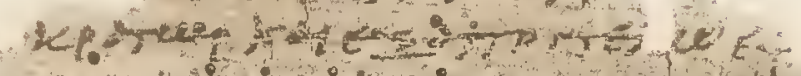

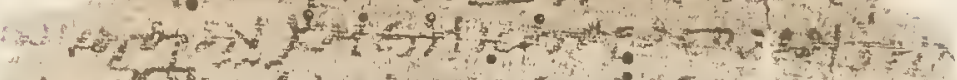

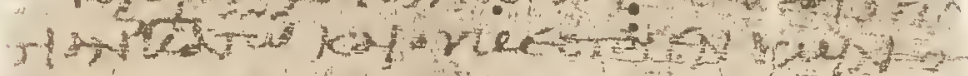

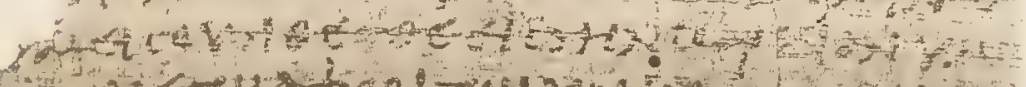

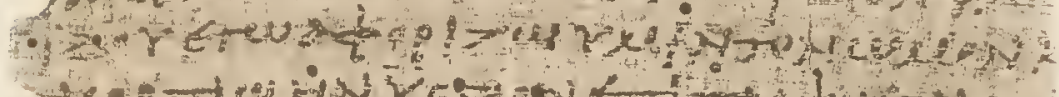

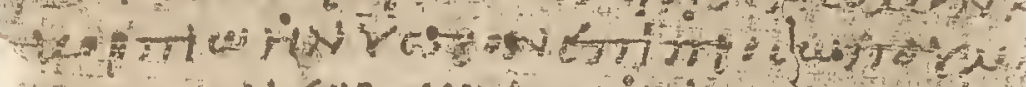
सि

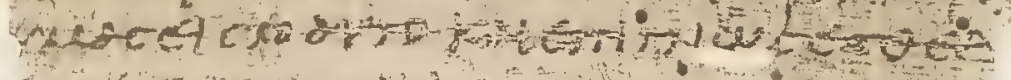

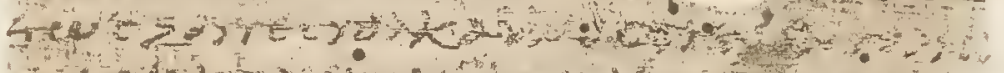

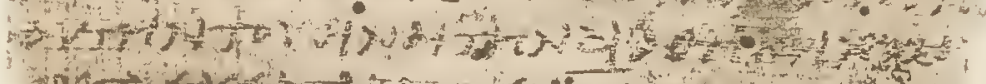

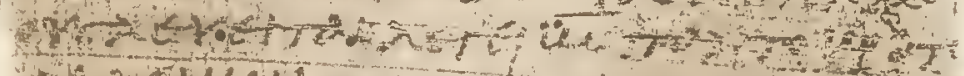

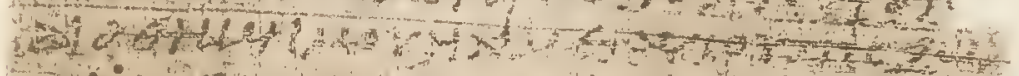

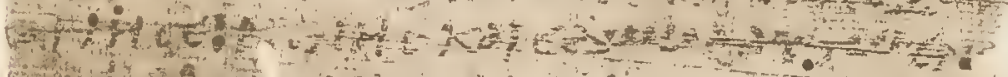

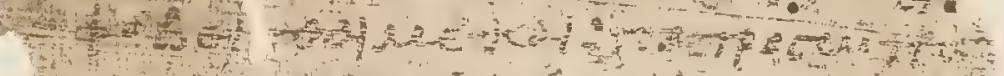

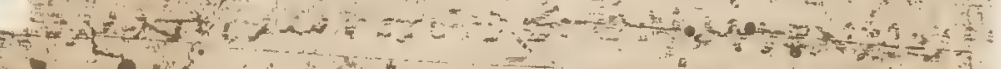

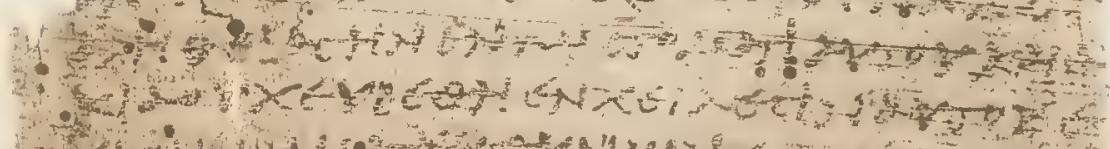

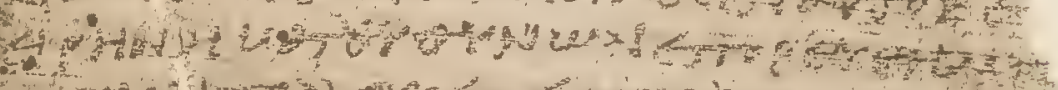

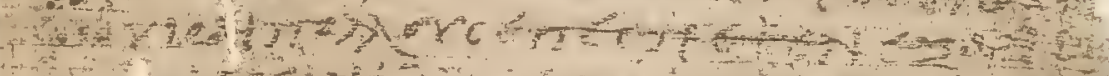

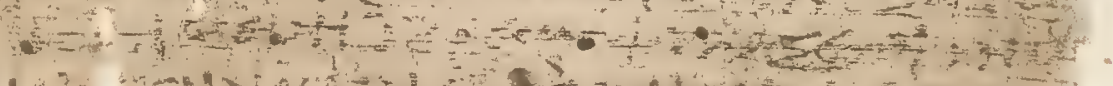

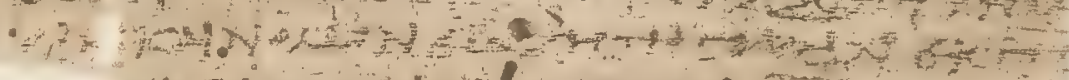

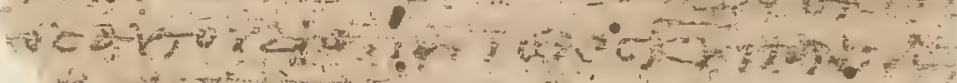
-

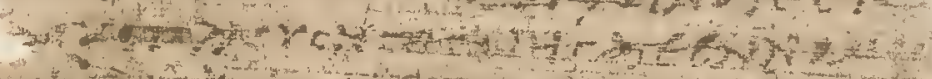

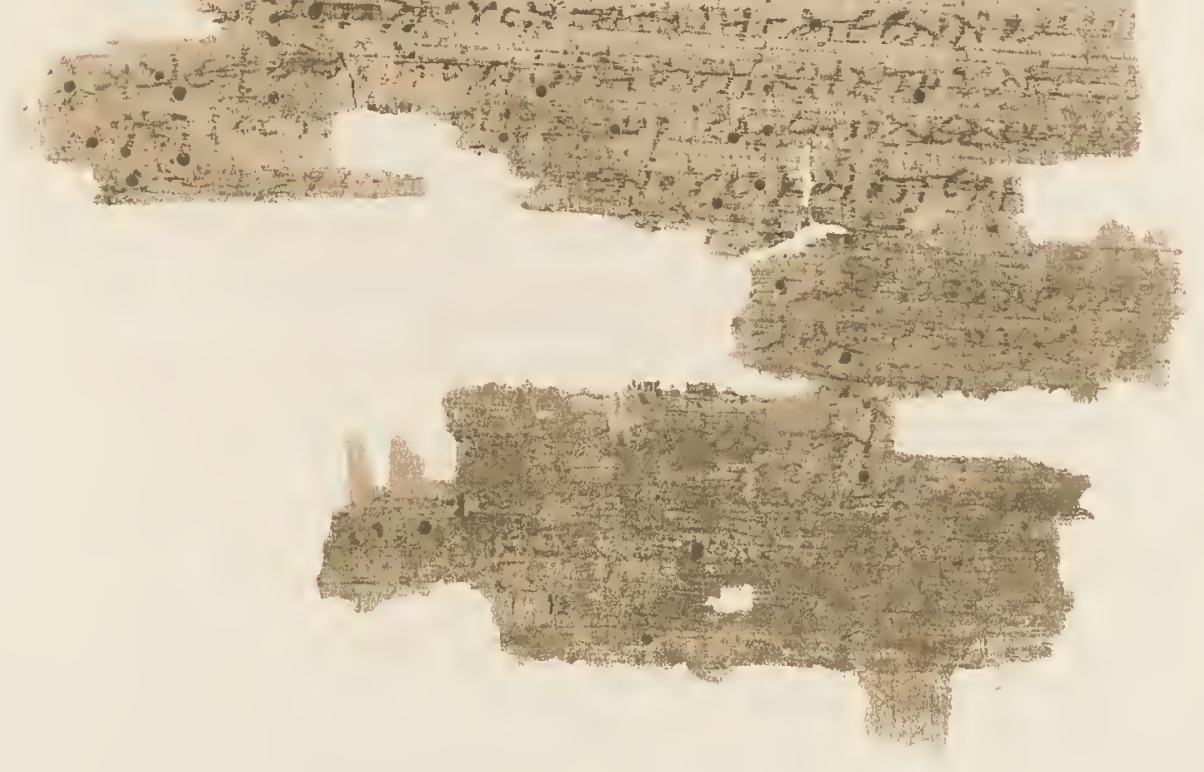




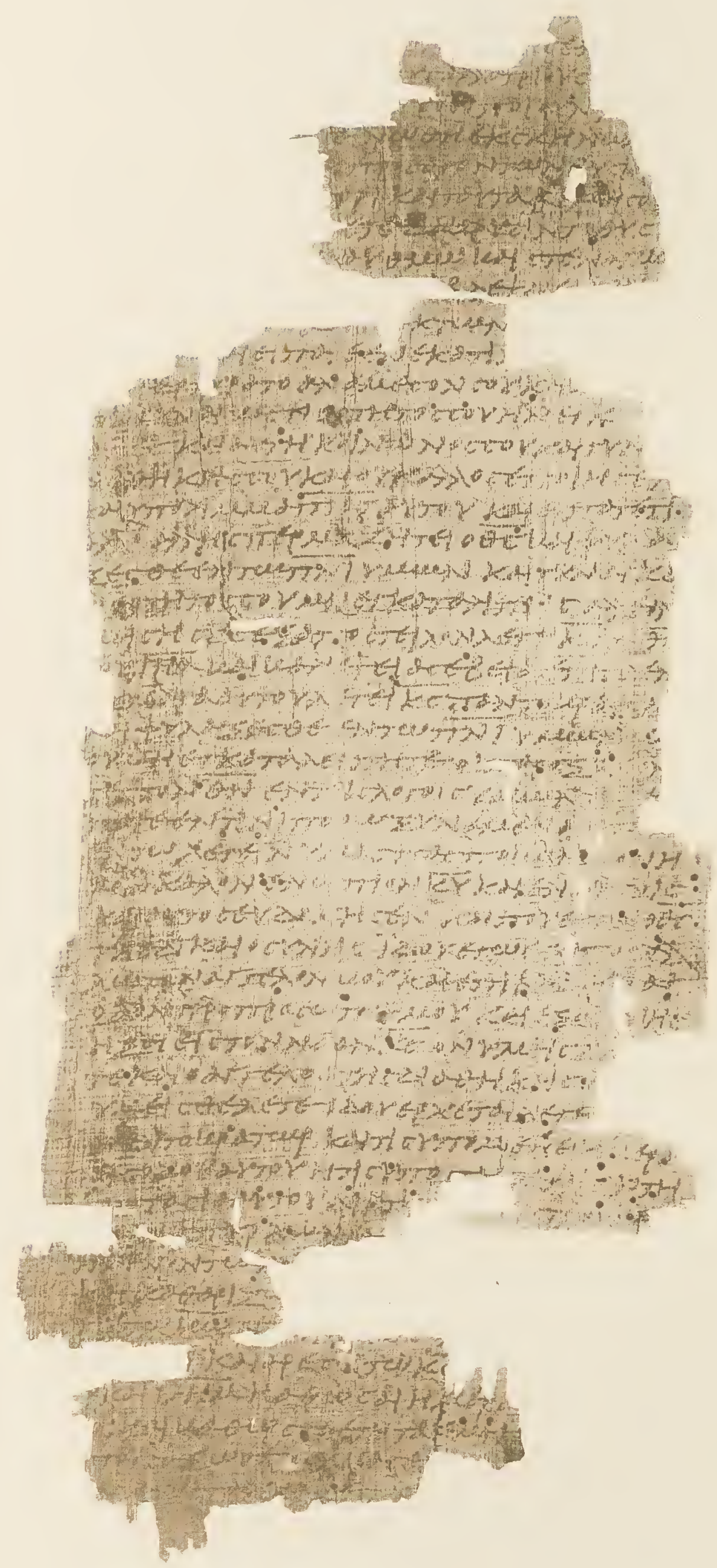




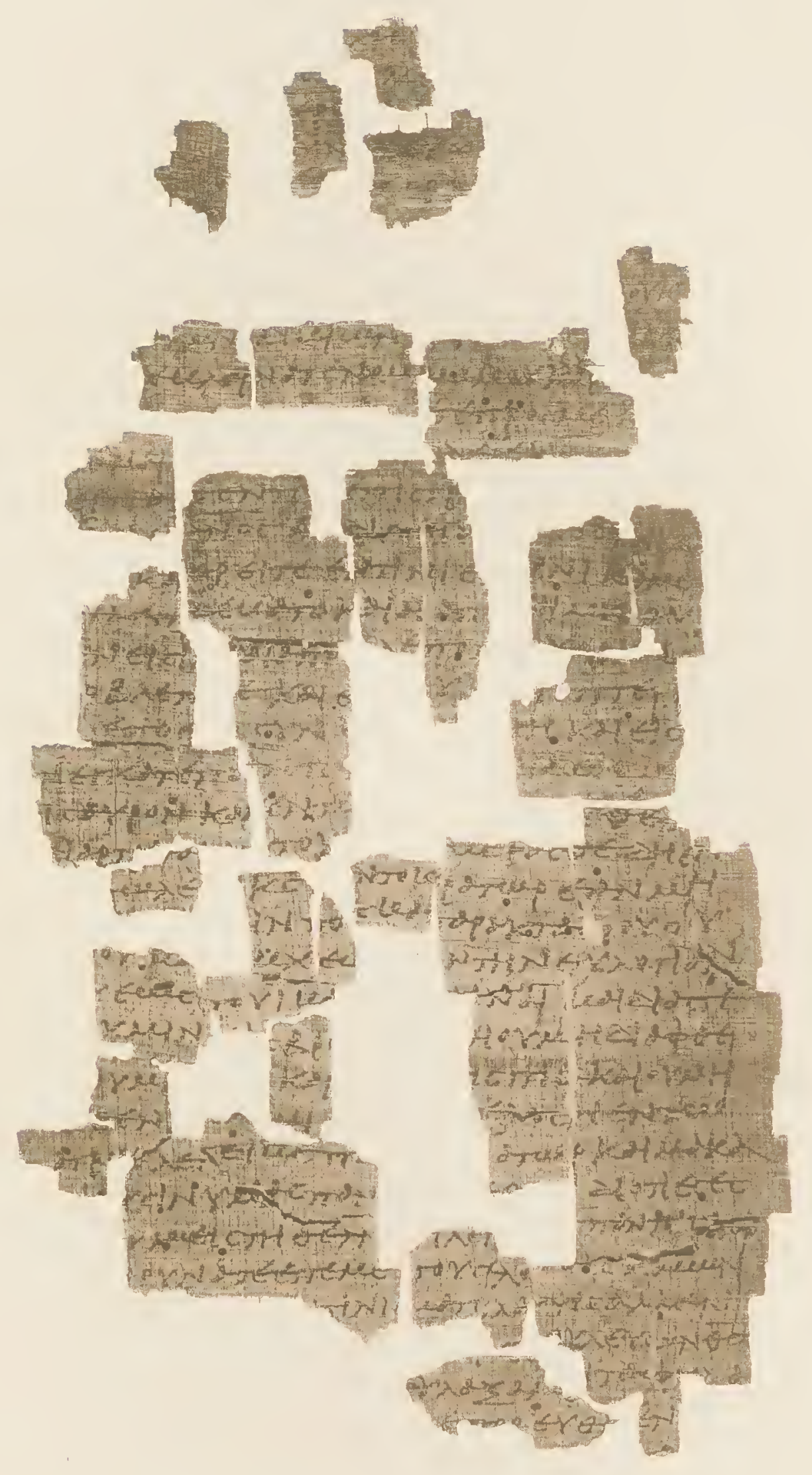

MaLACHAS iii, 5-16. 

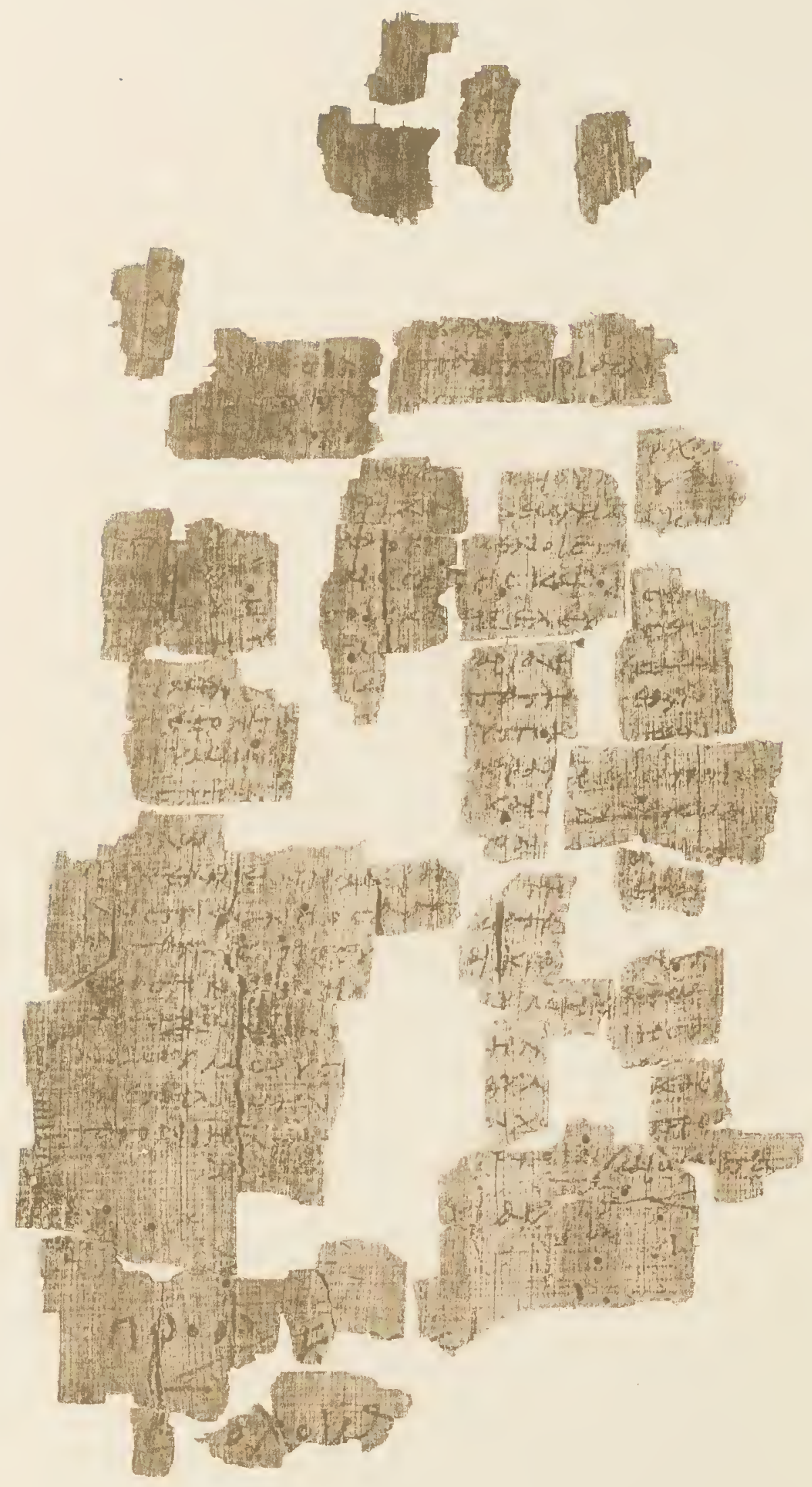

Malacinas iii, 16 -iv, 6. 


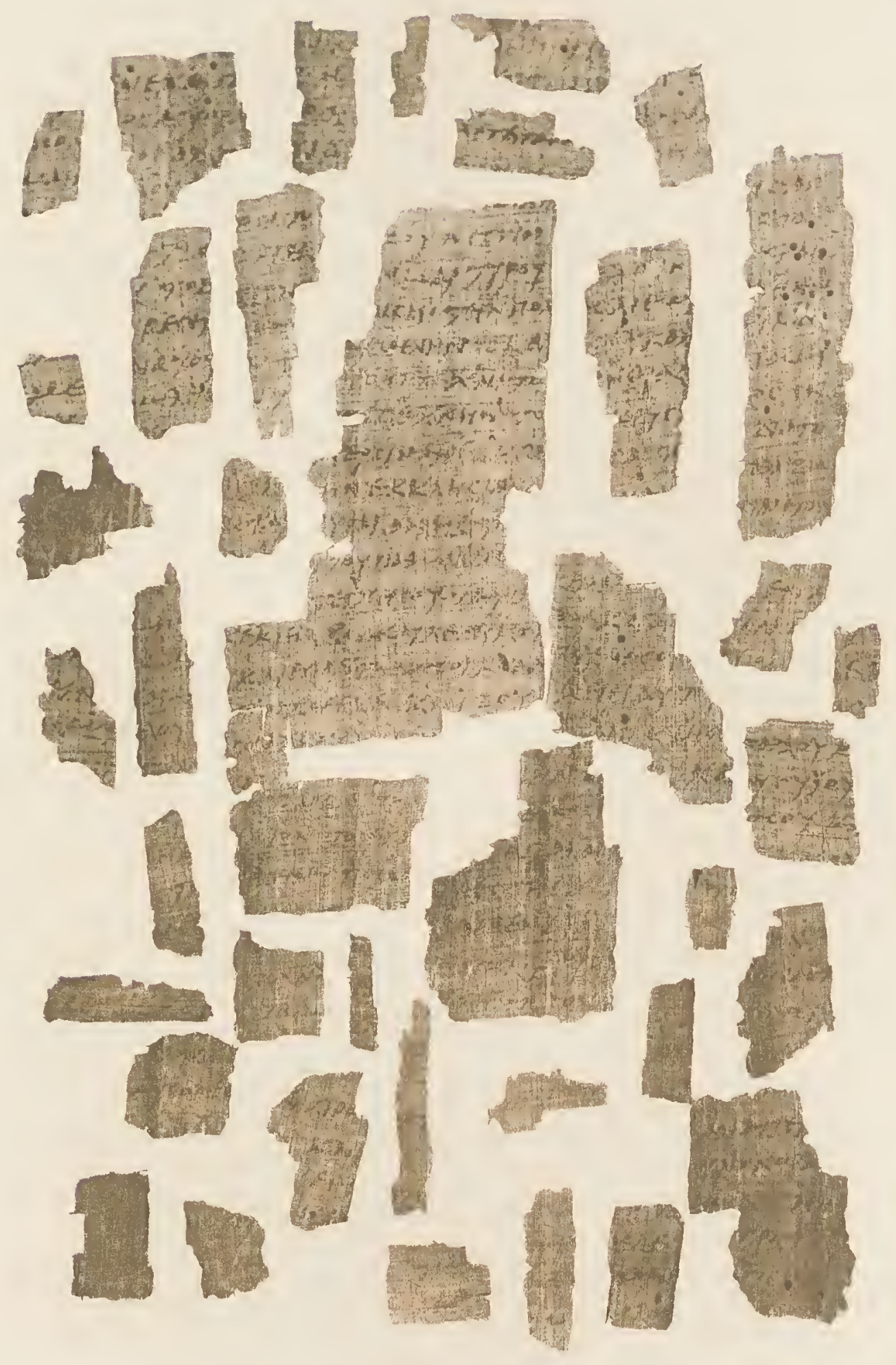




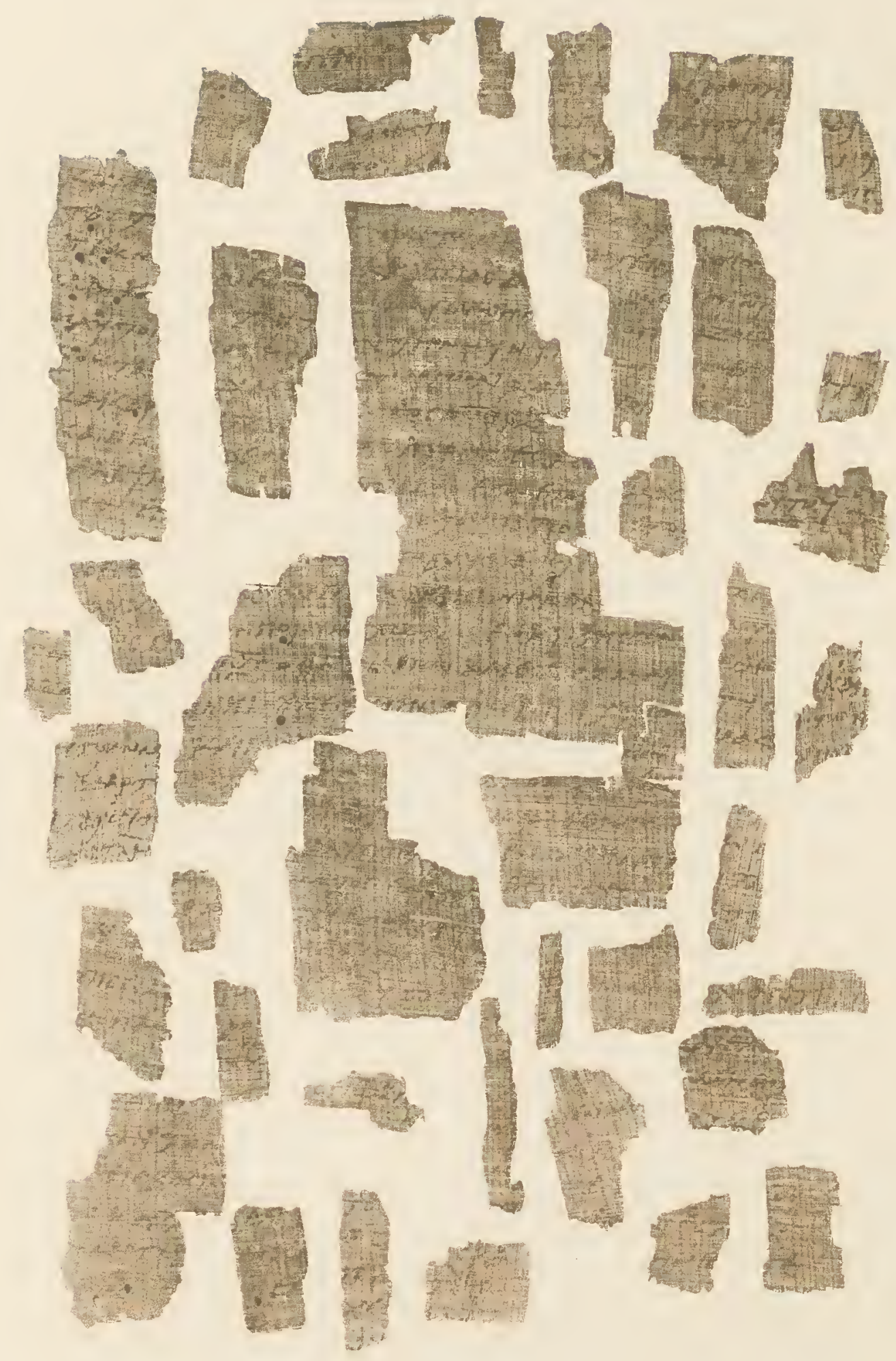




\section{PART II}

THE FACSIMILE OF THE BERLIN FRAGMENT OF

GENESIS 

A

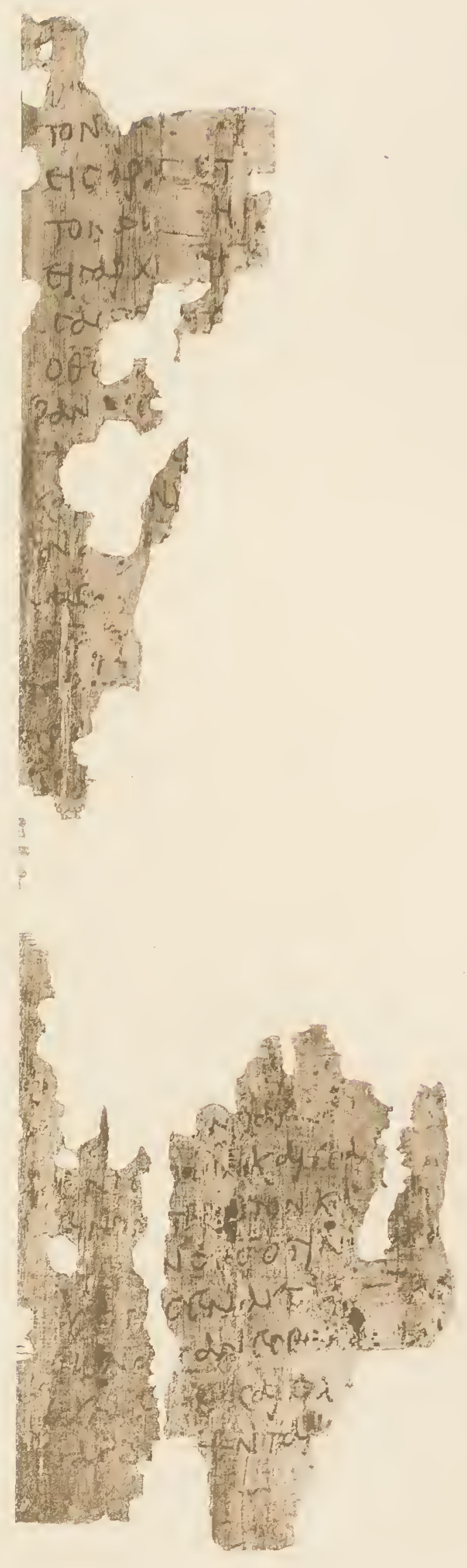



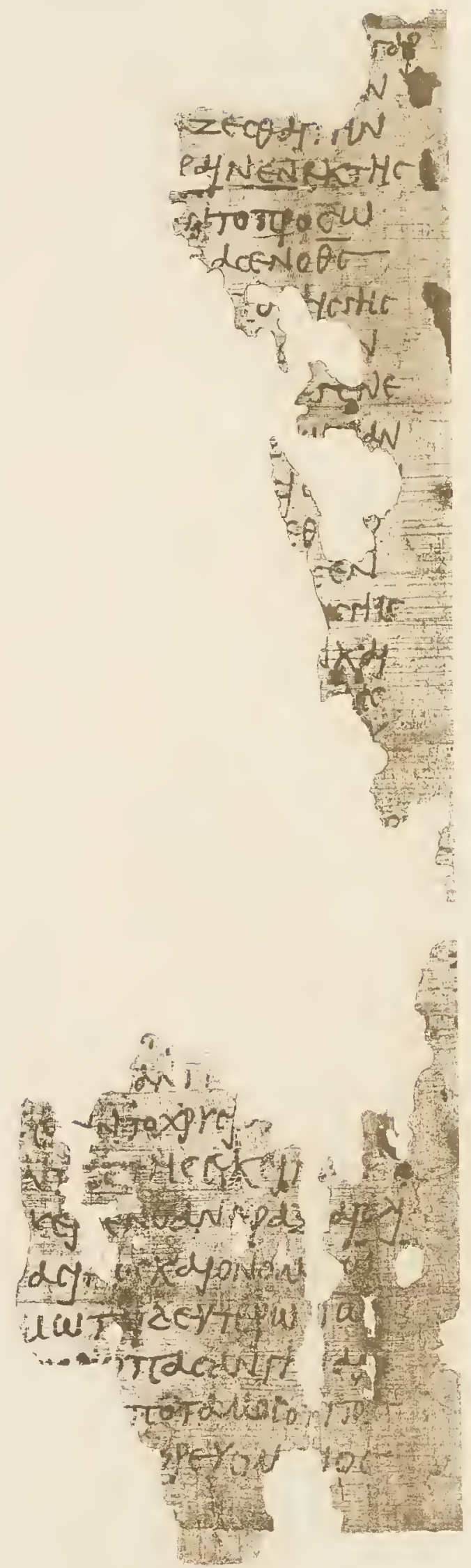

Genesis ii, $5-14$. 

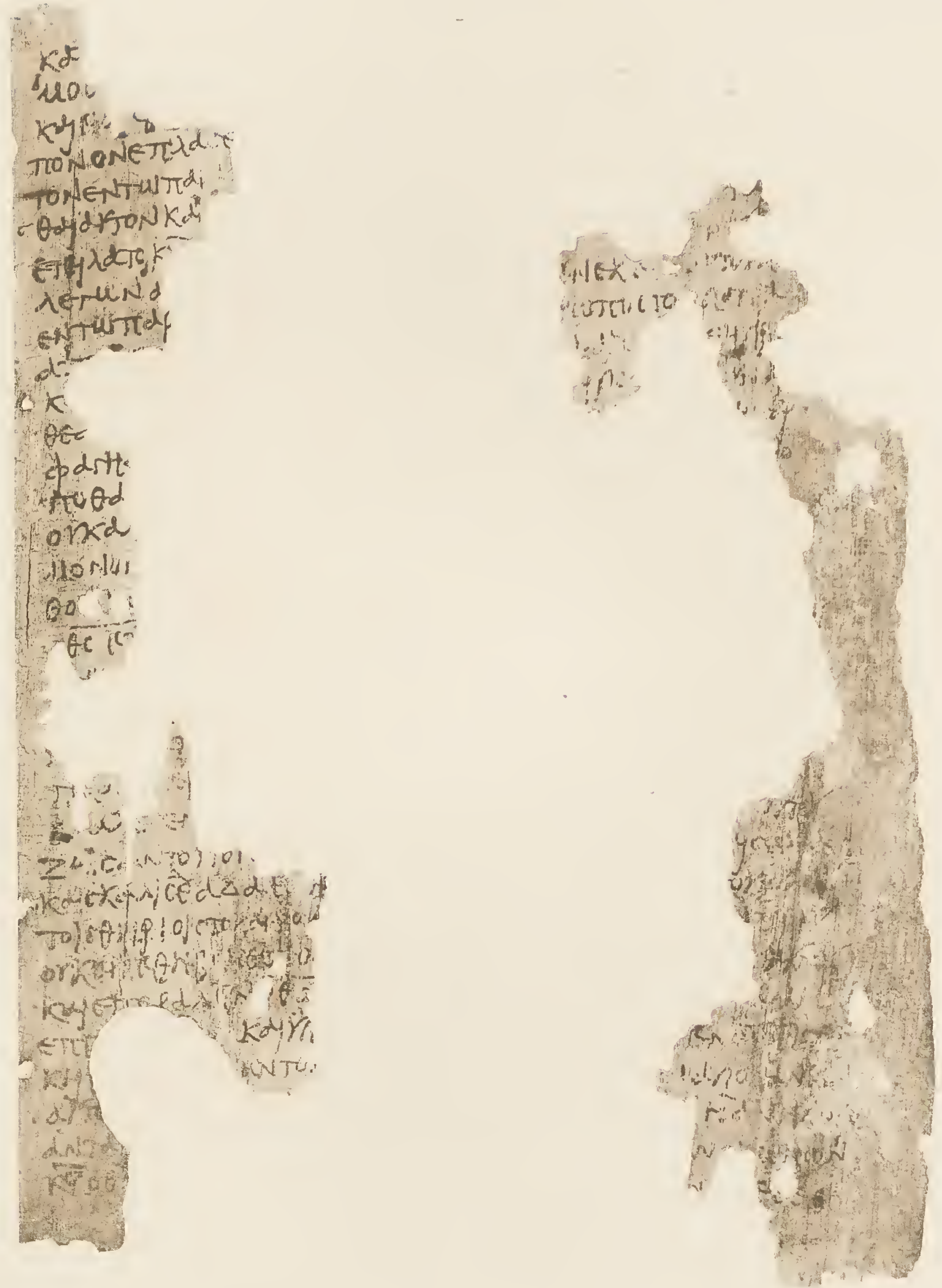

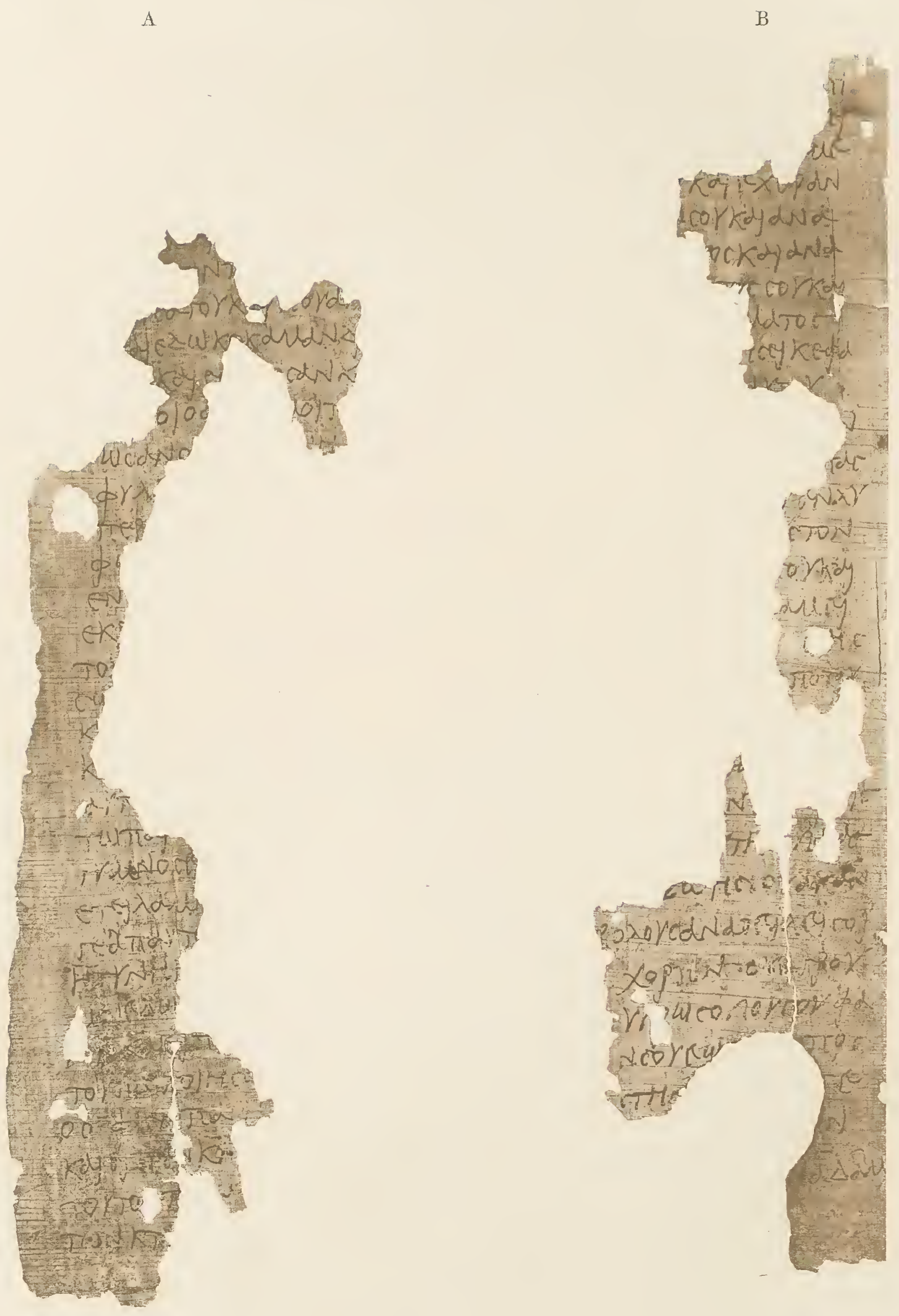

Genesis iii, $6-14$. iii, $14-20$. 

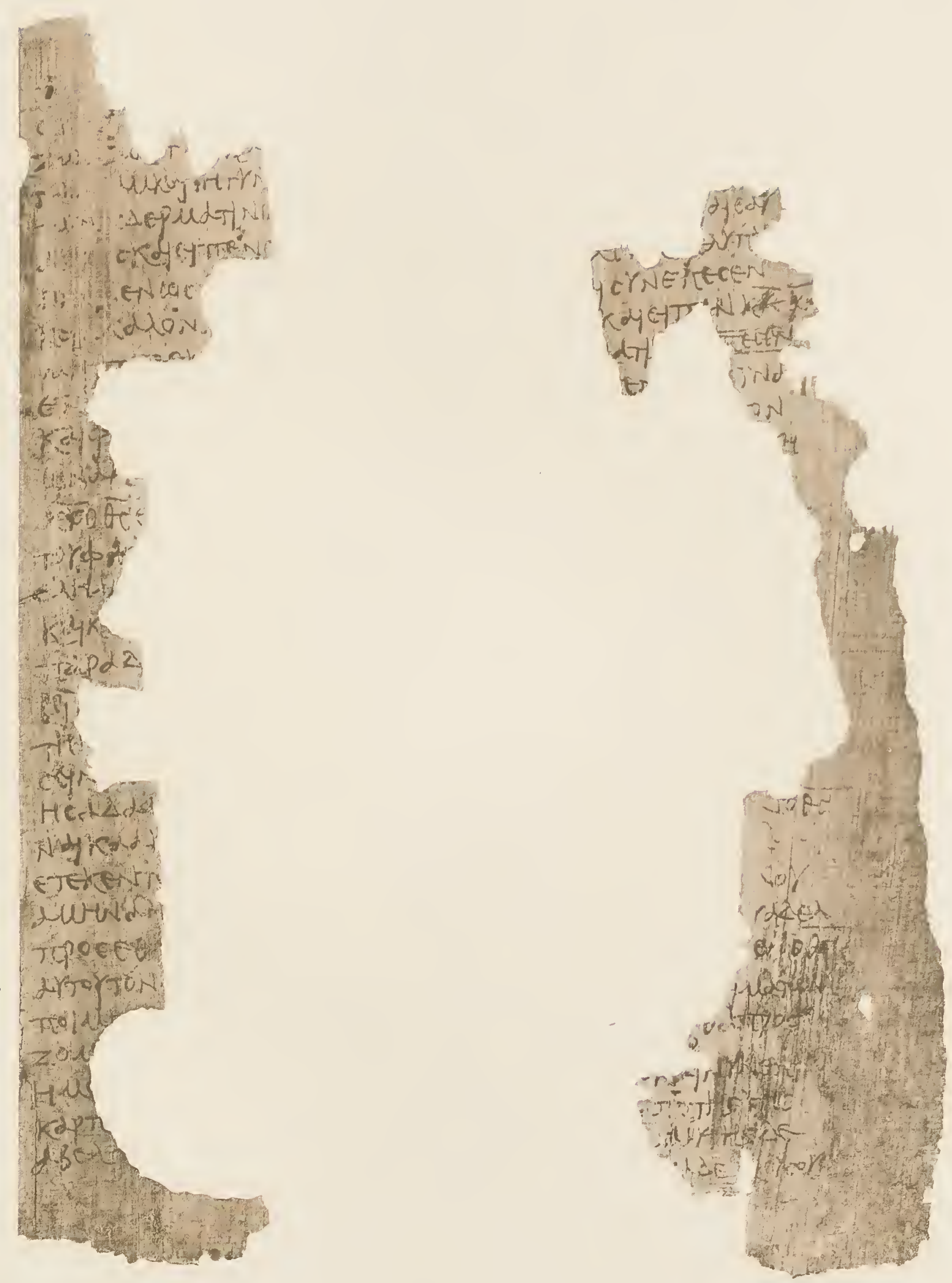

Gexests iii, 20-iv, 4. ir, $5-11$. 
A

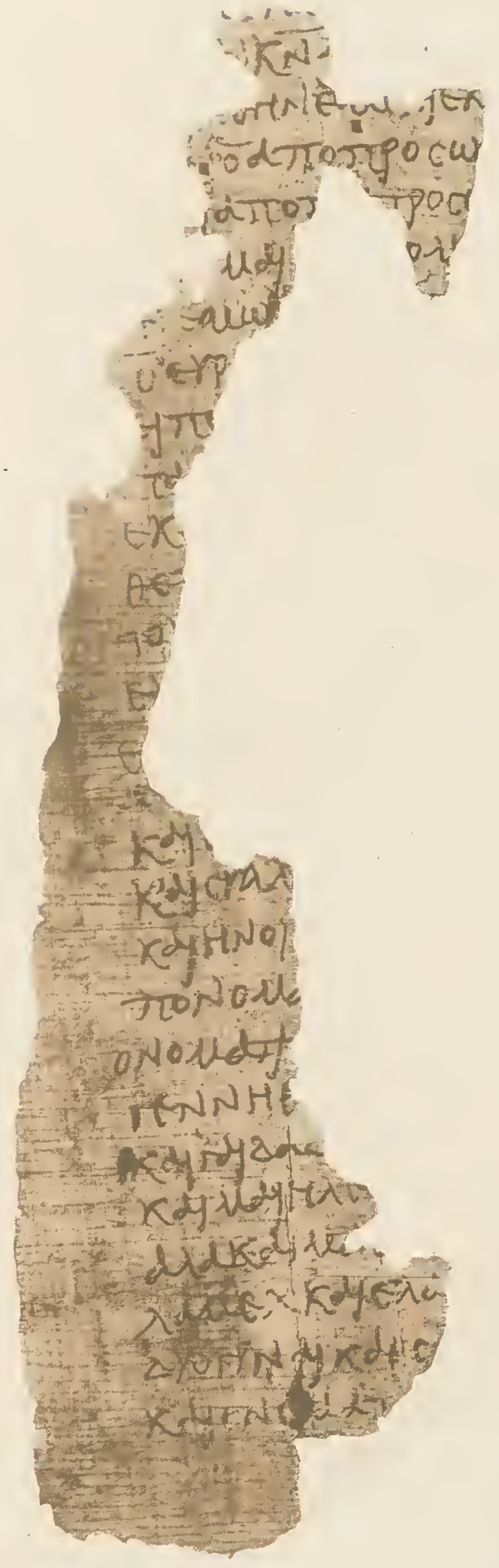

B

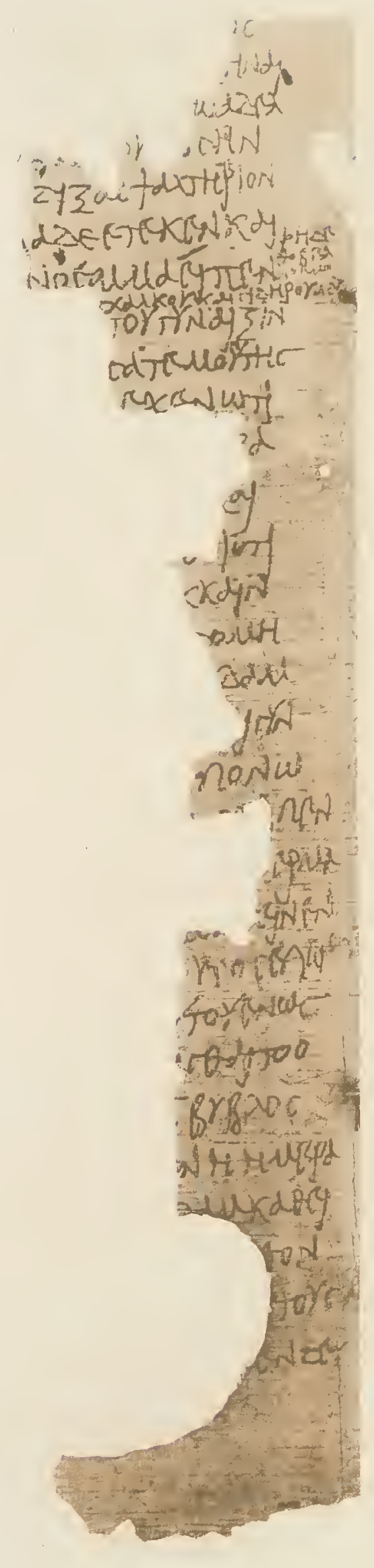

Genesis iv, $13-19 . \quad$ iv, $20-\mathrm{r}, 3$. 

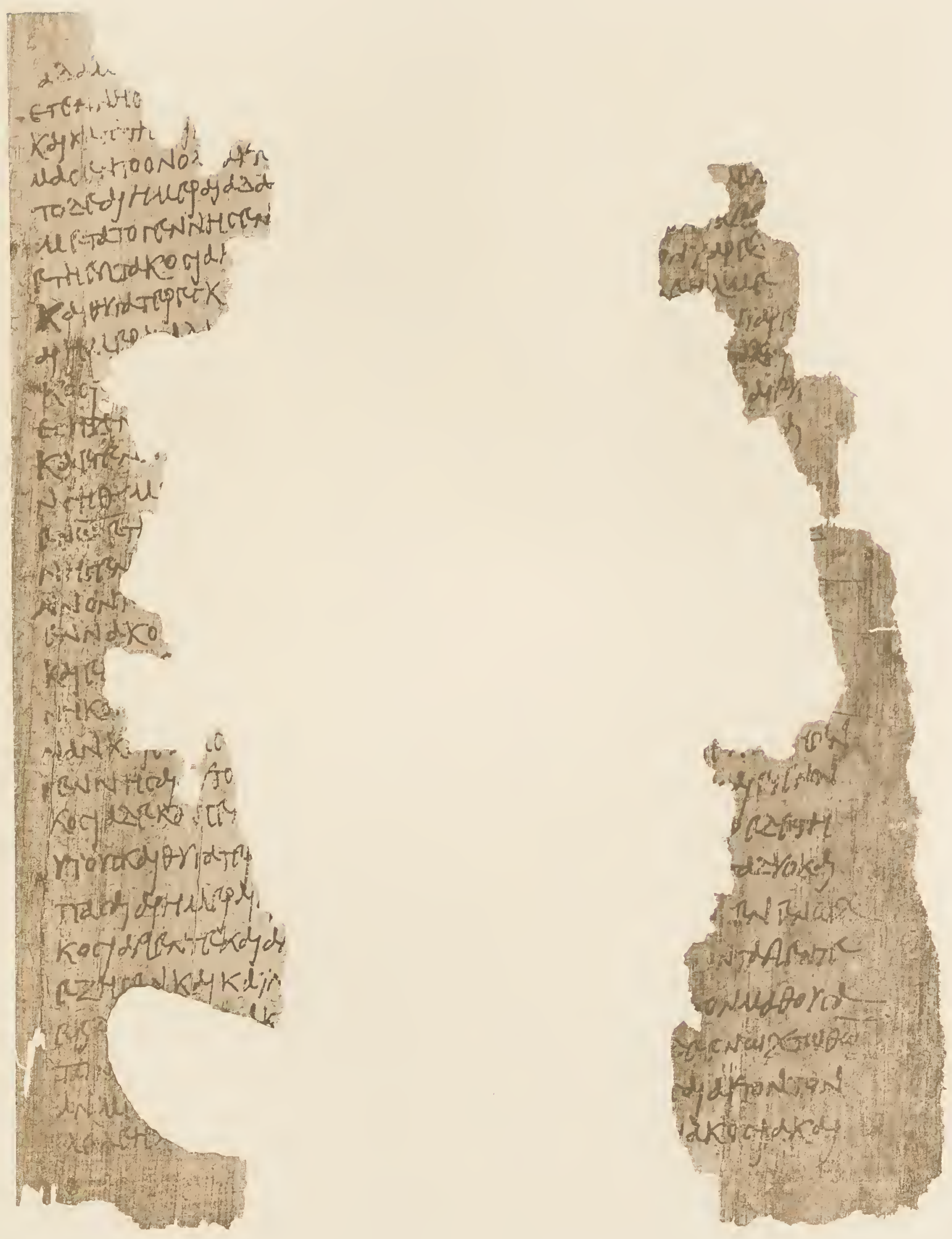

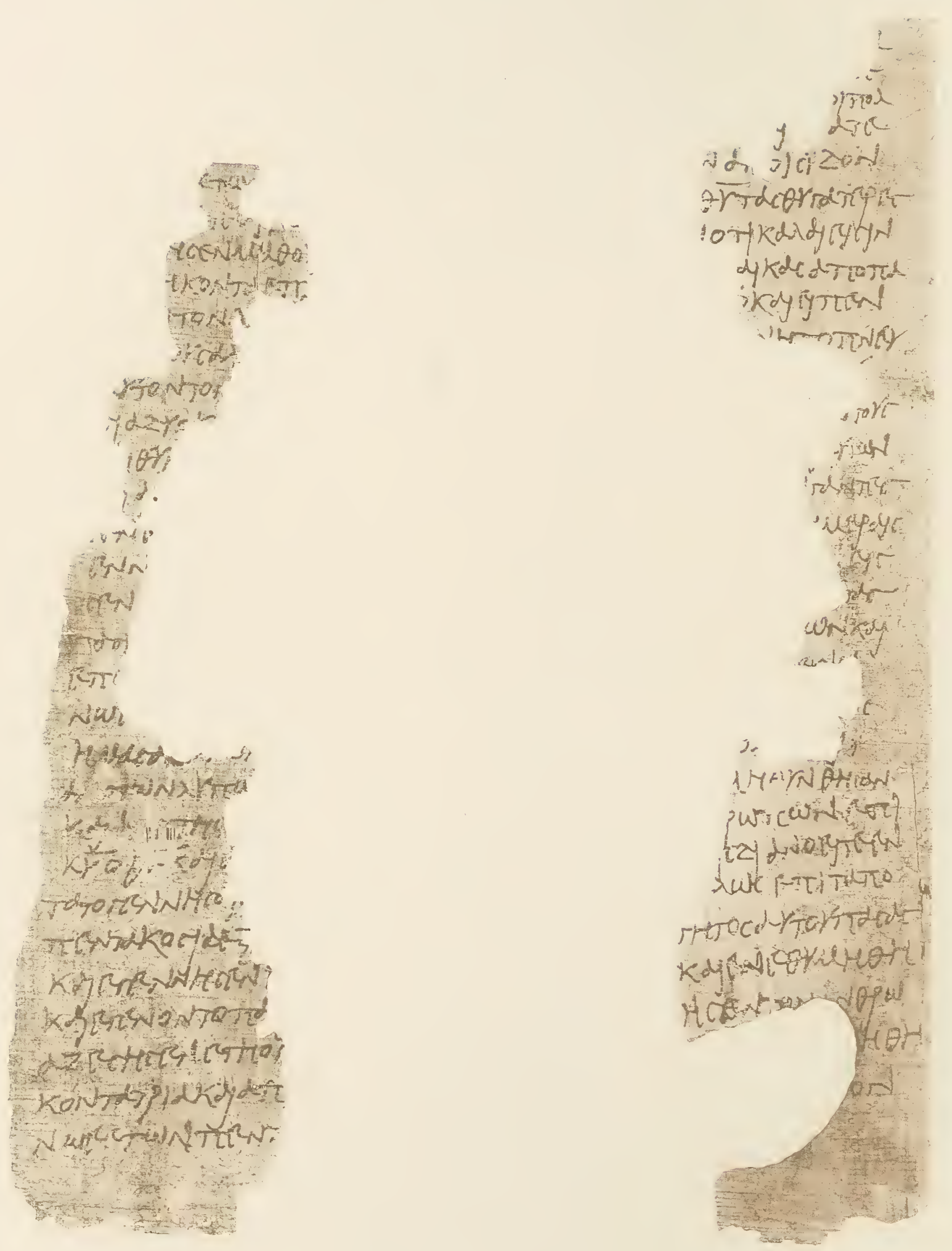

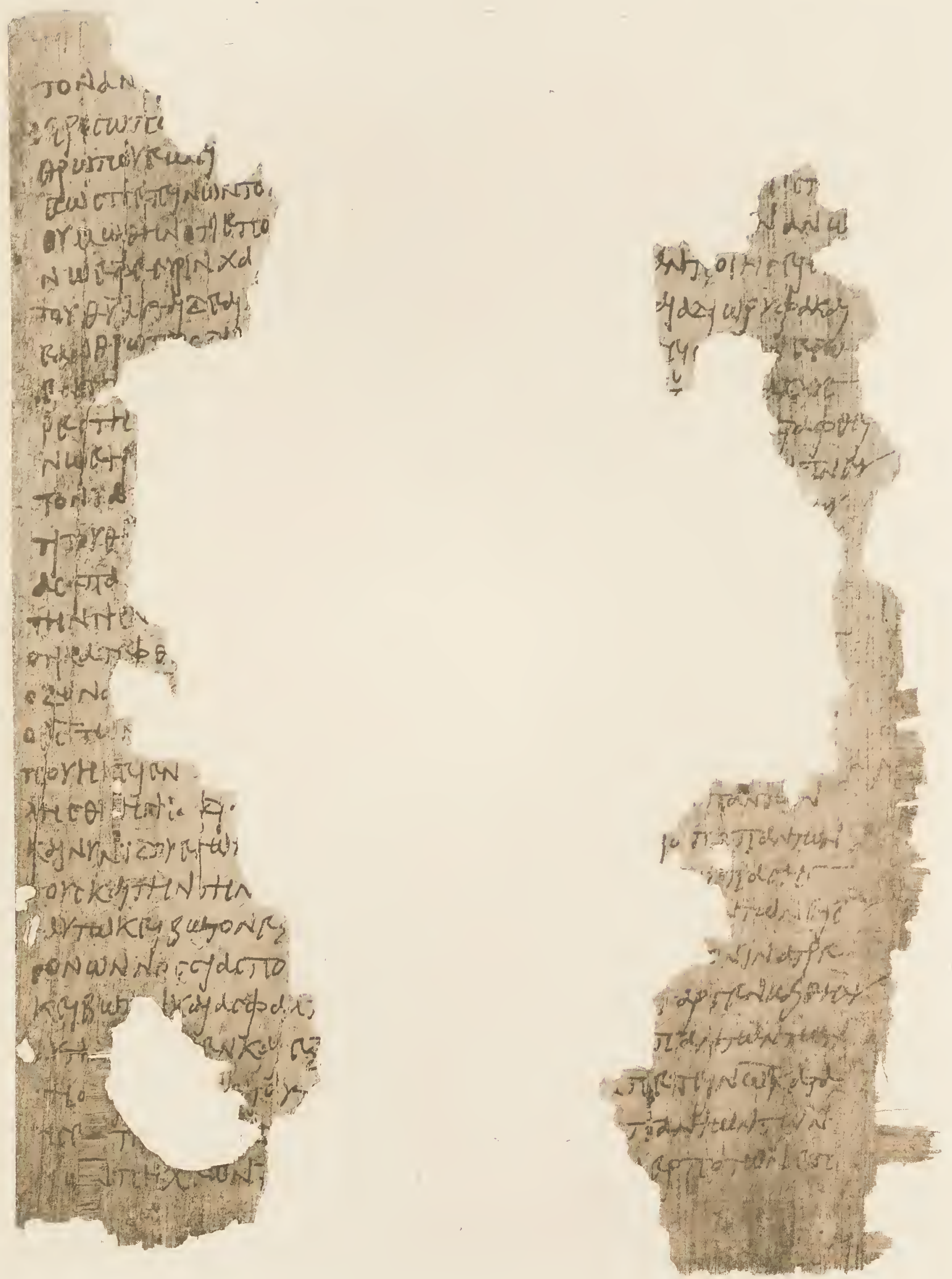

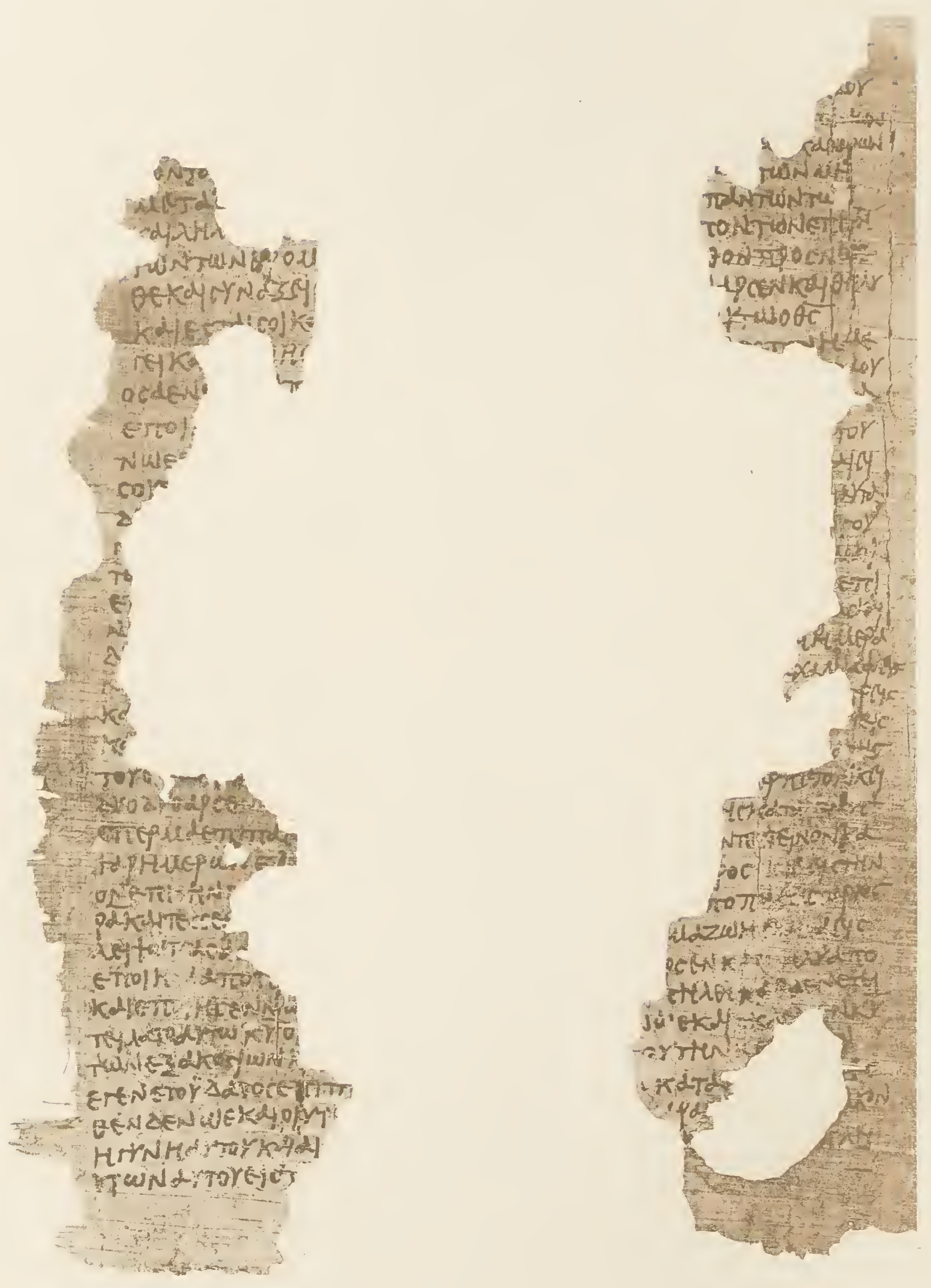

Gevesis vi, 20 - vii, $7 . \quad$ rii, $7-17$. 

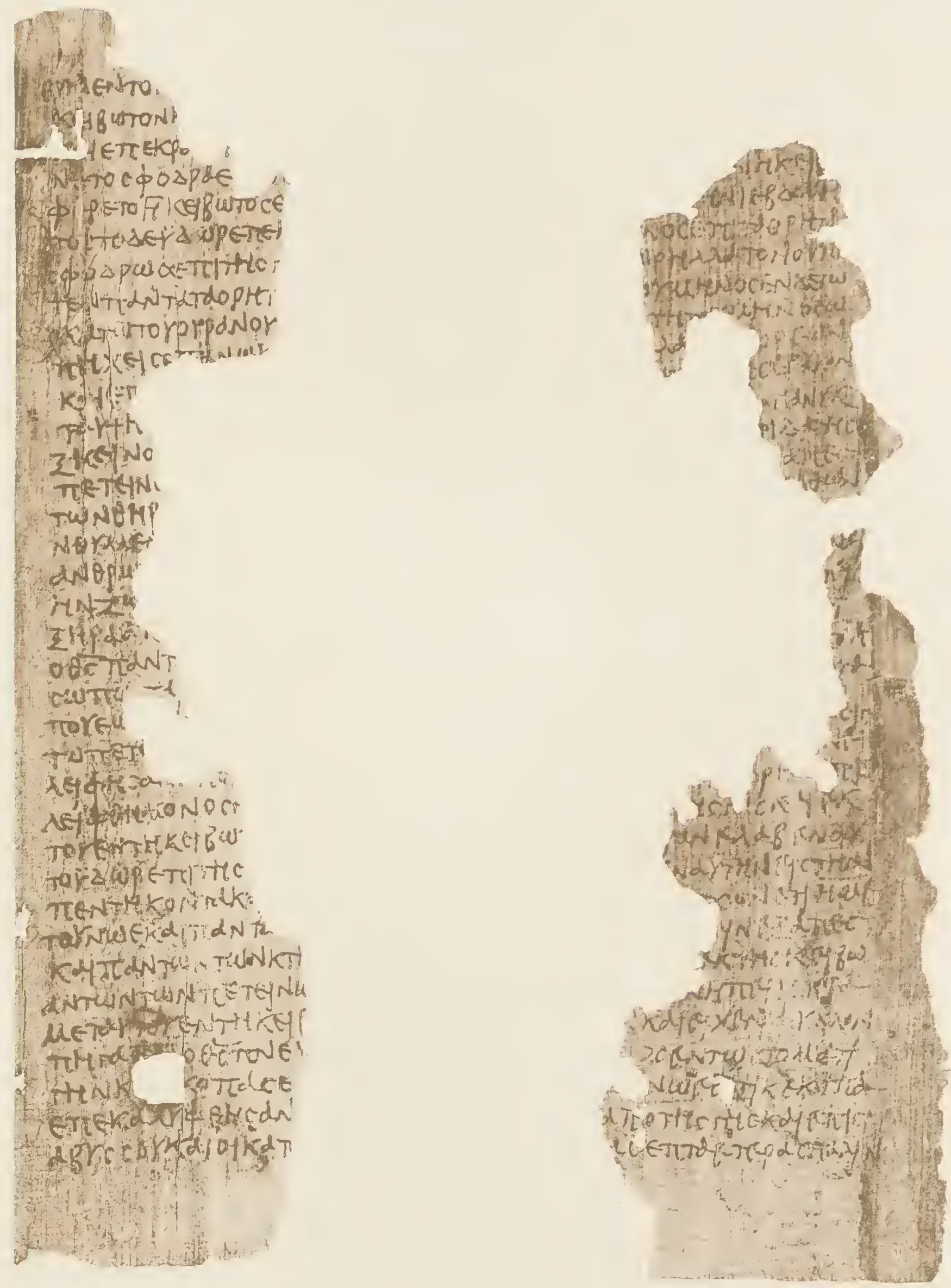

Genesrs vii, 17 - riii, 2. viii, 4-12. 

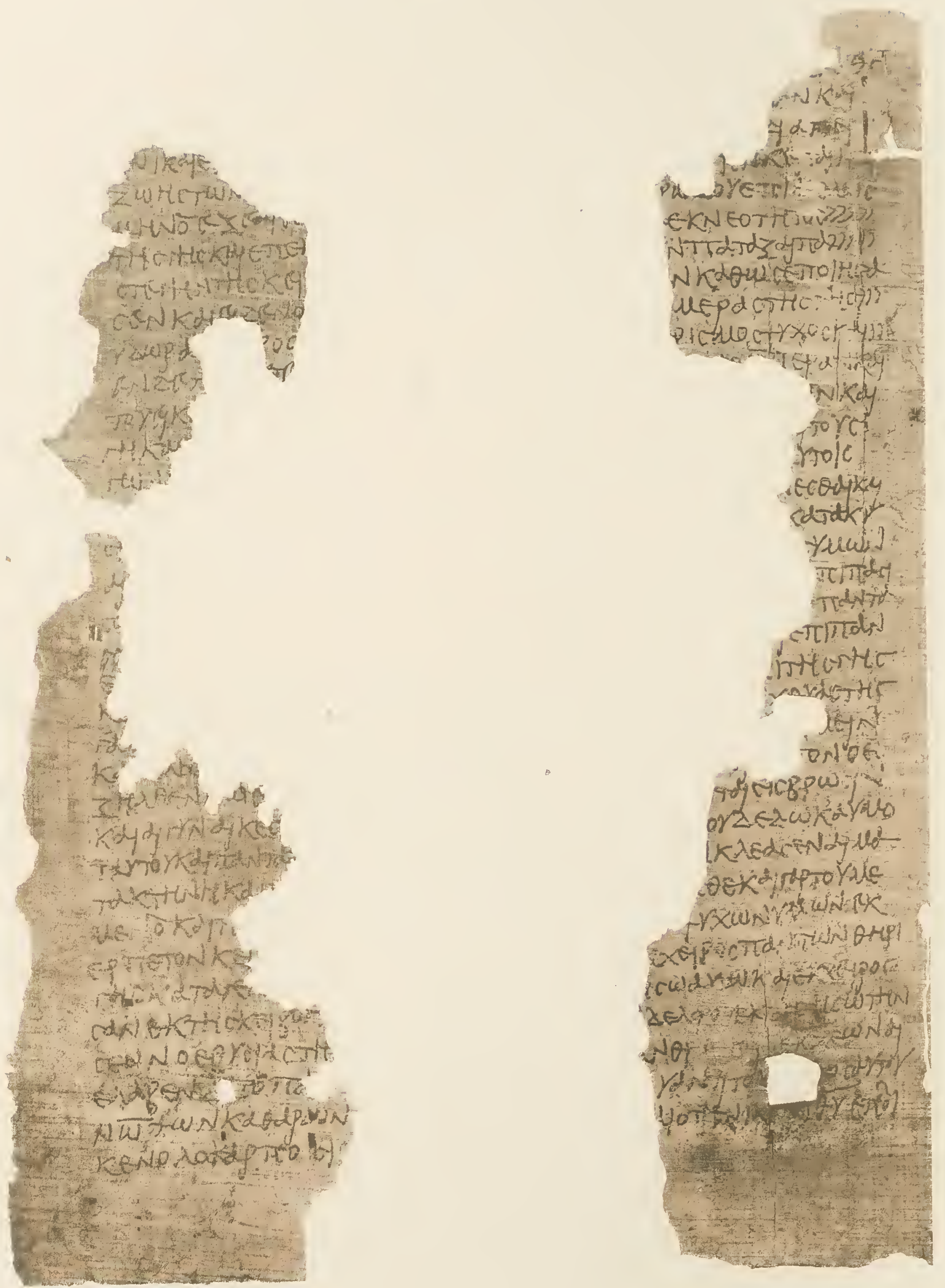

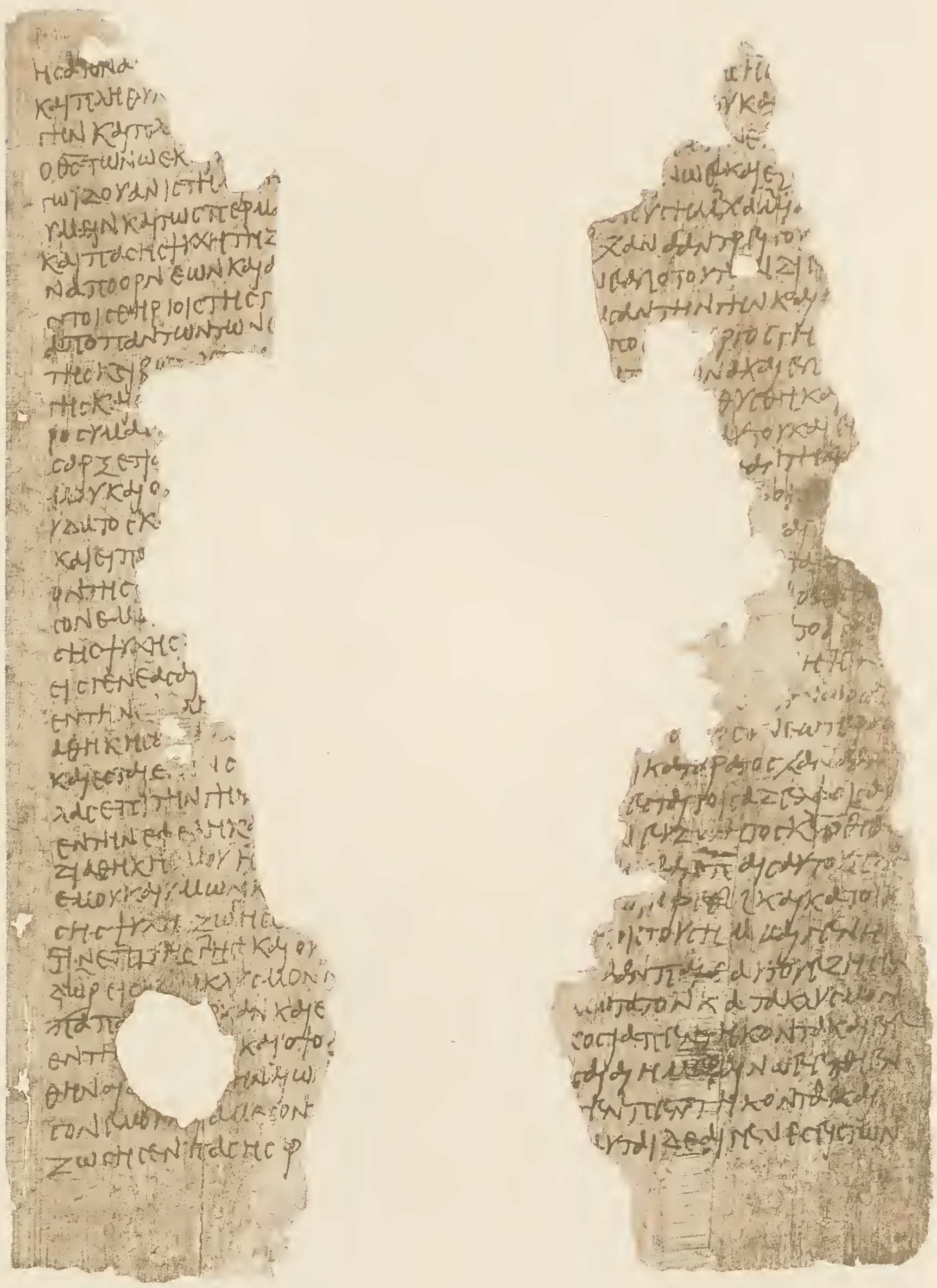

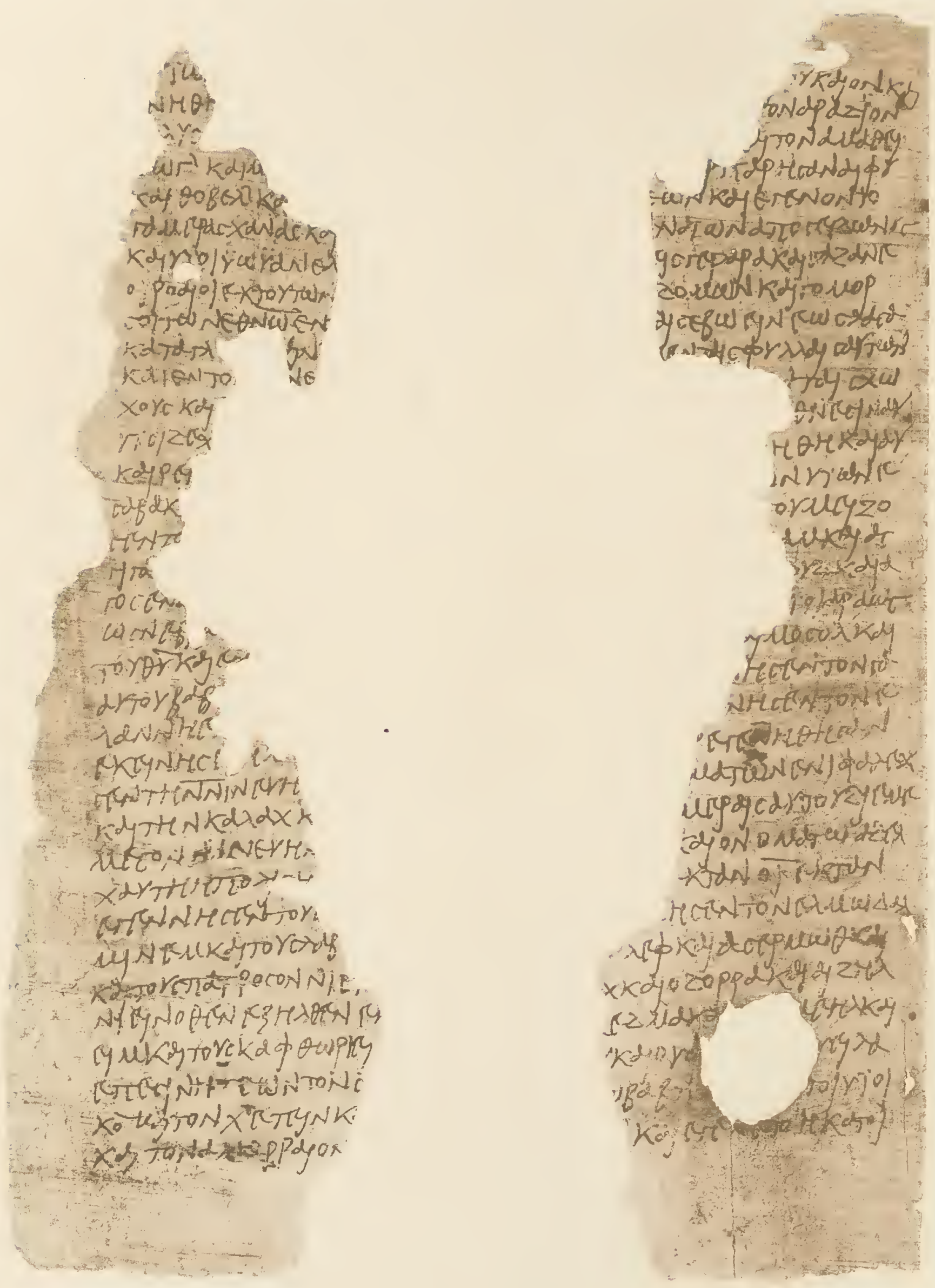

Genesis x, $1-16 . \quad \mathrm{x}, 1 \mathrm{i}-30$. 

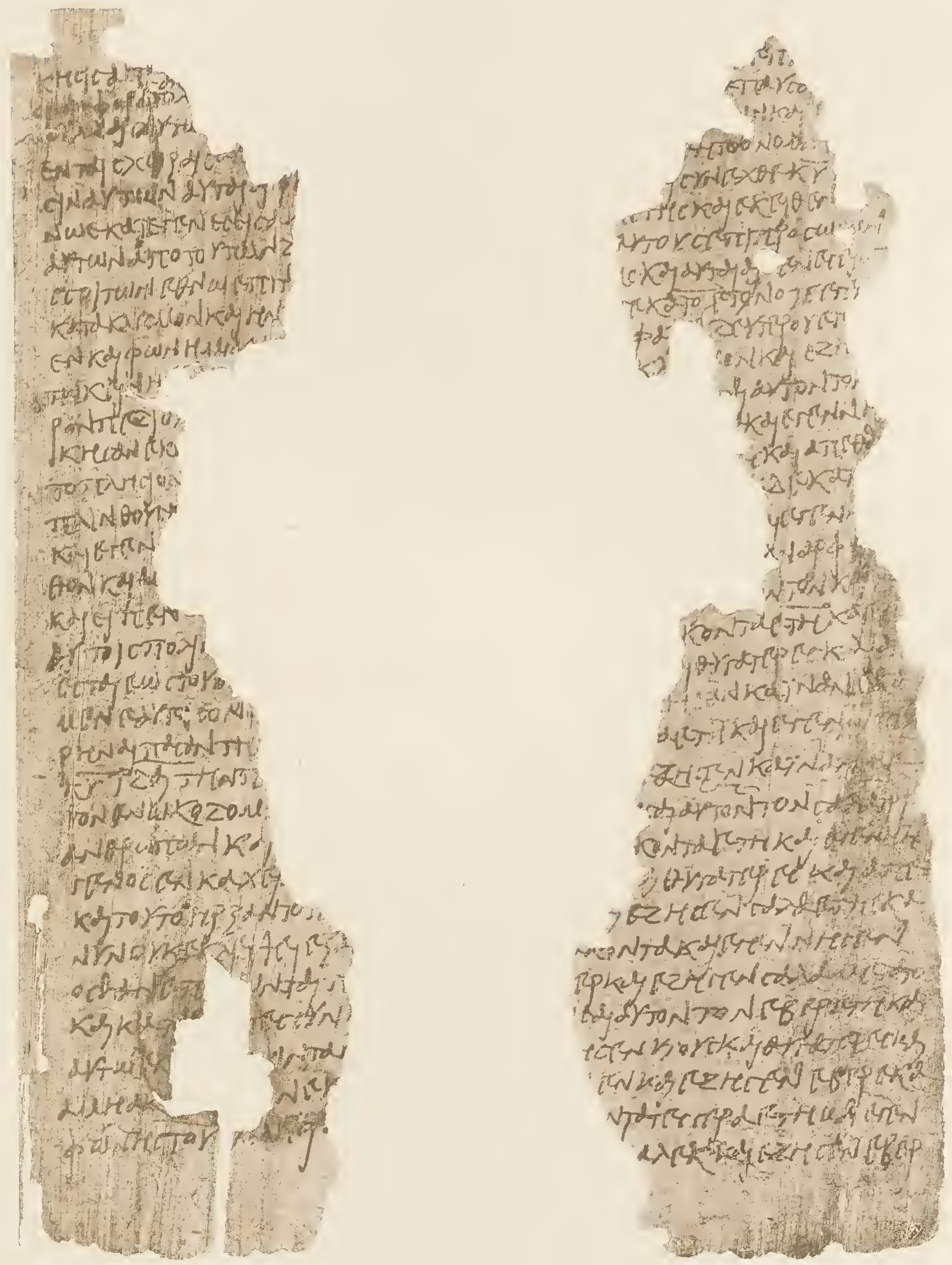

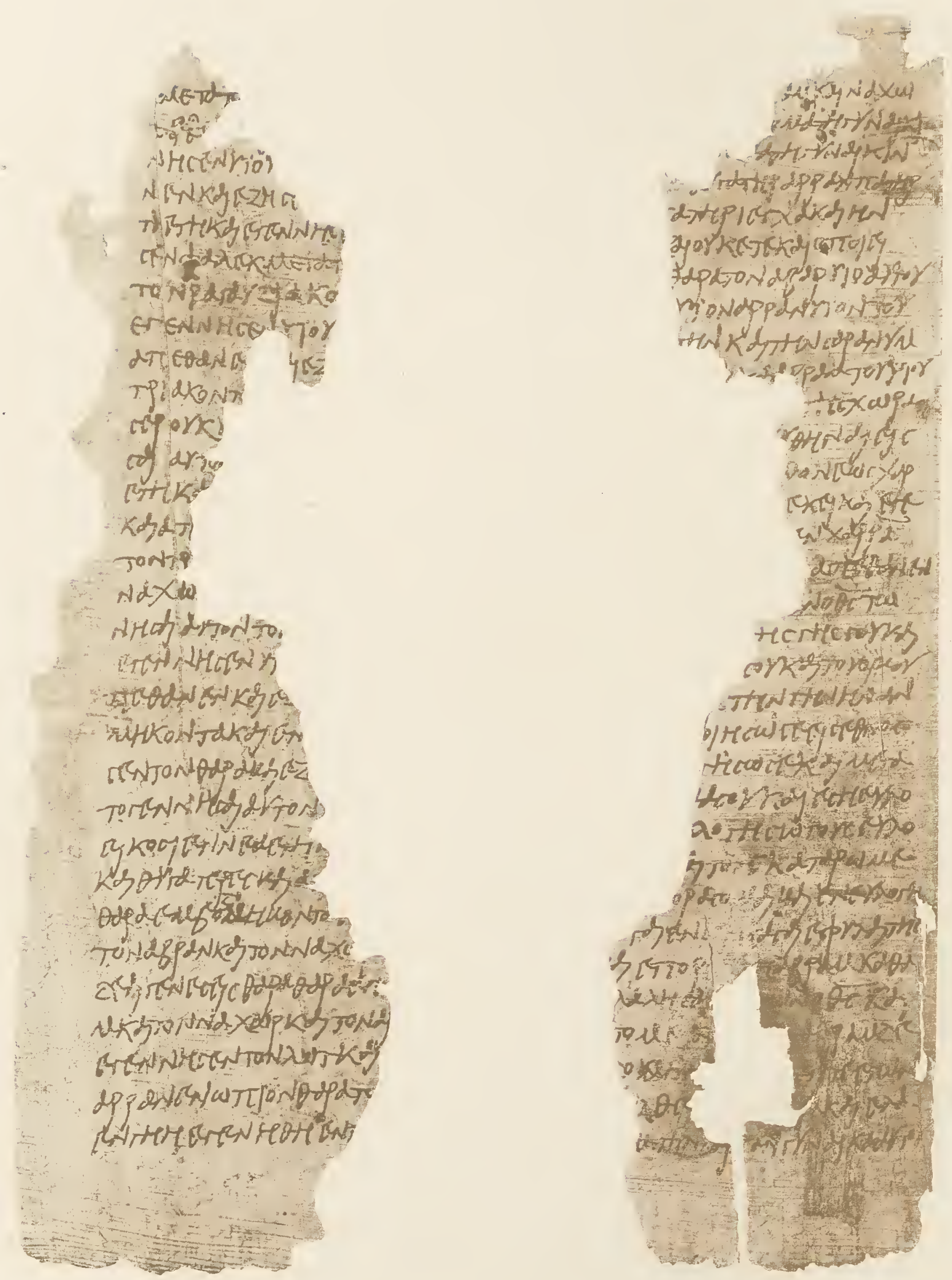

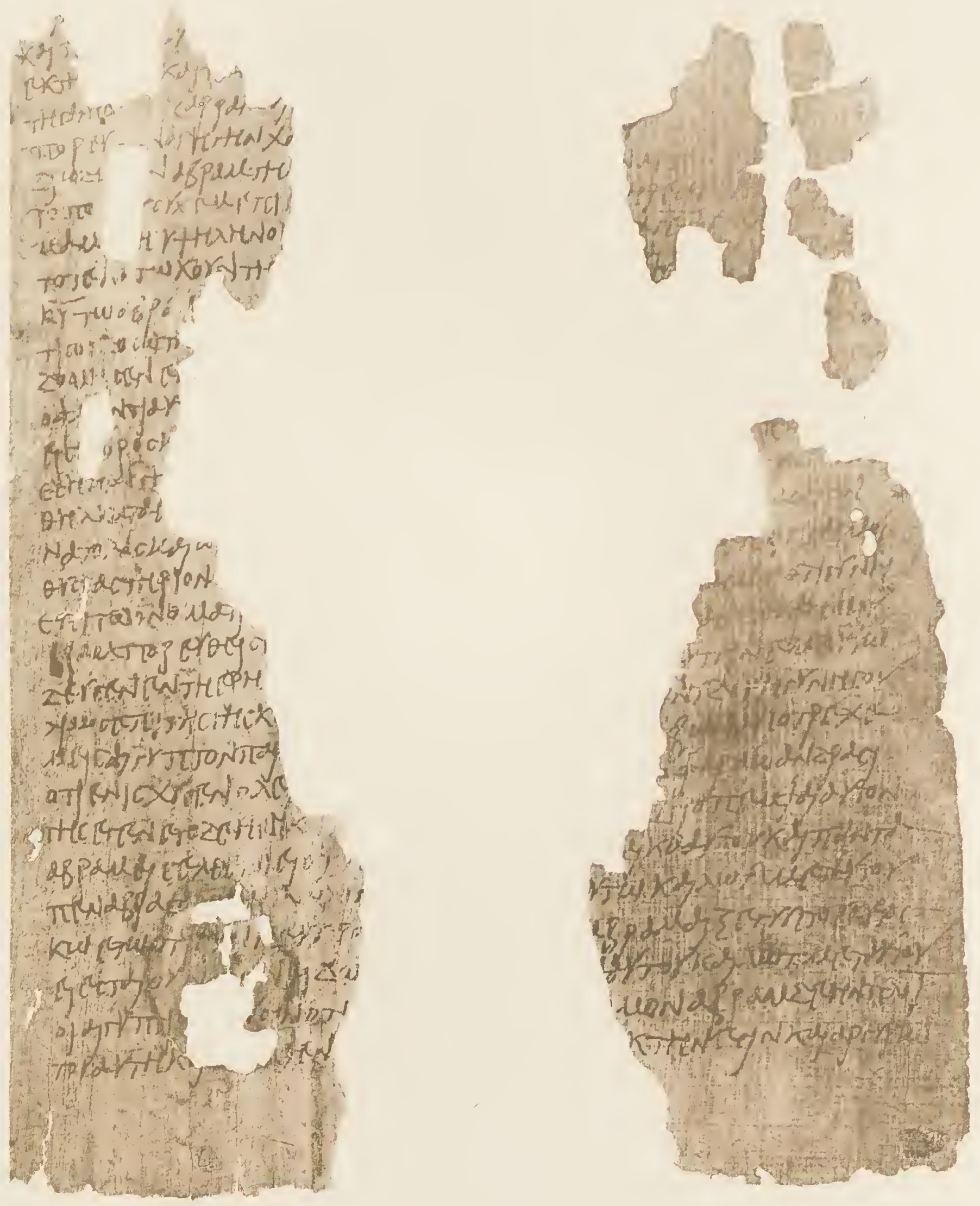

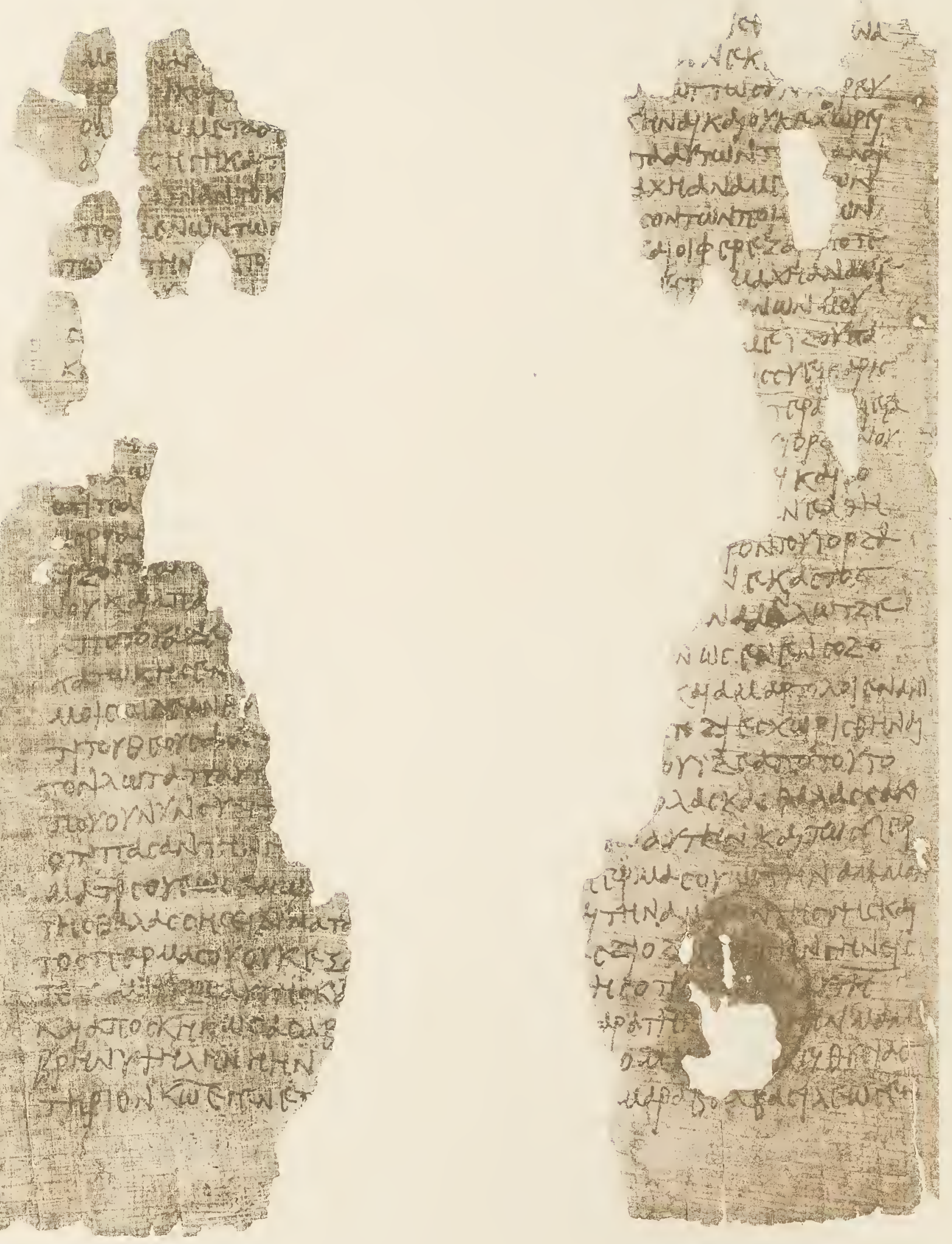

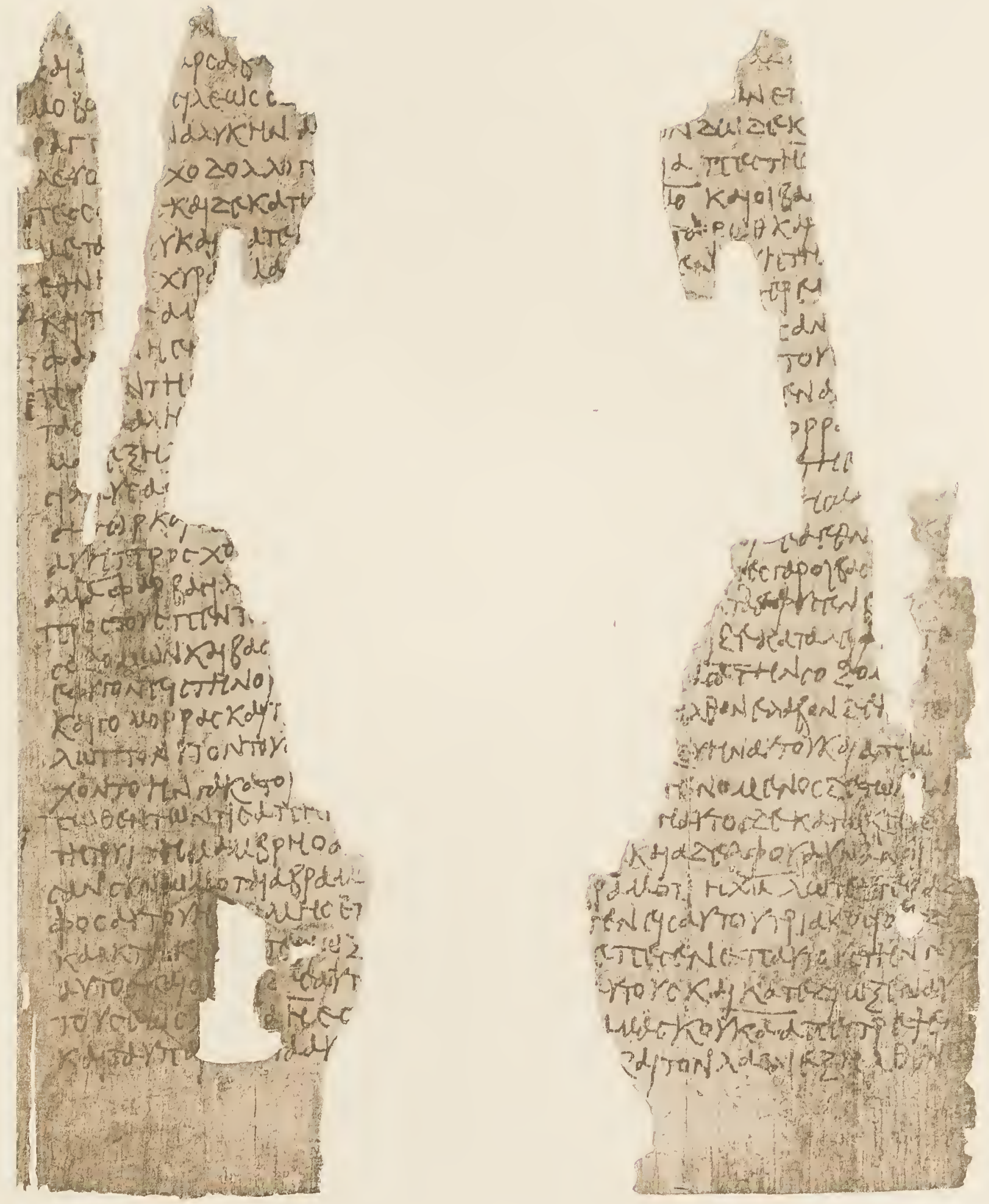

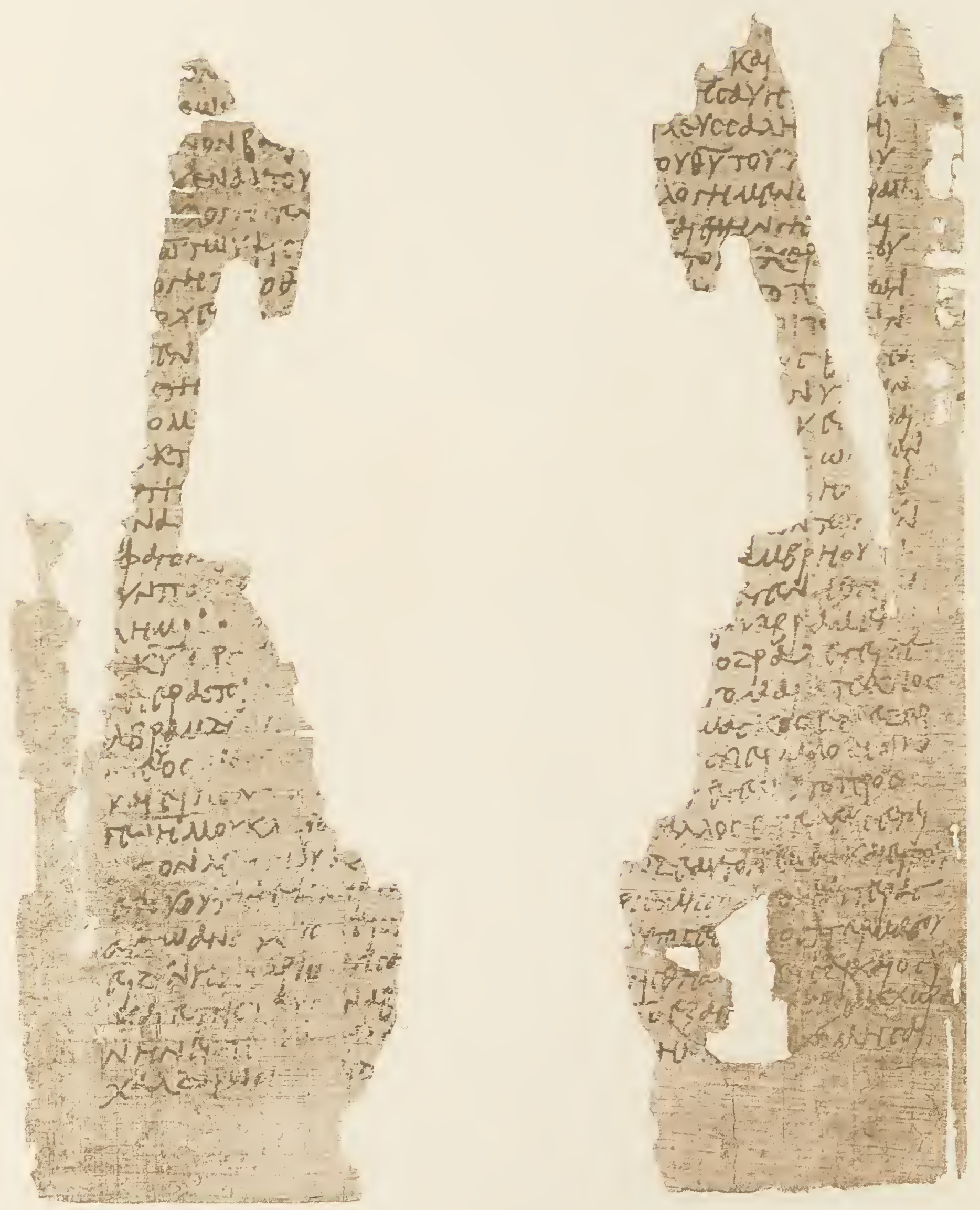


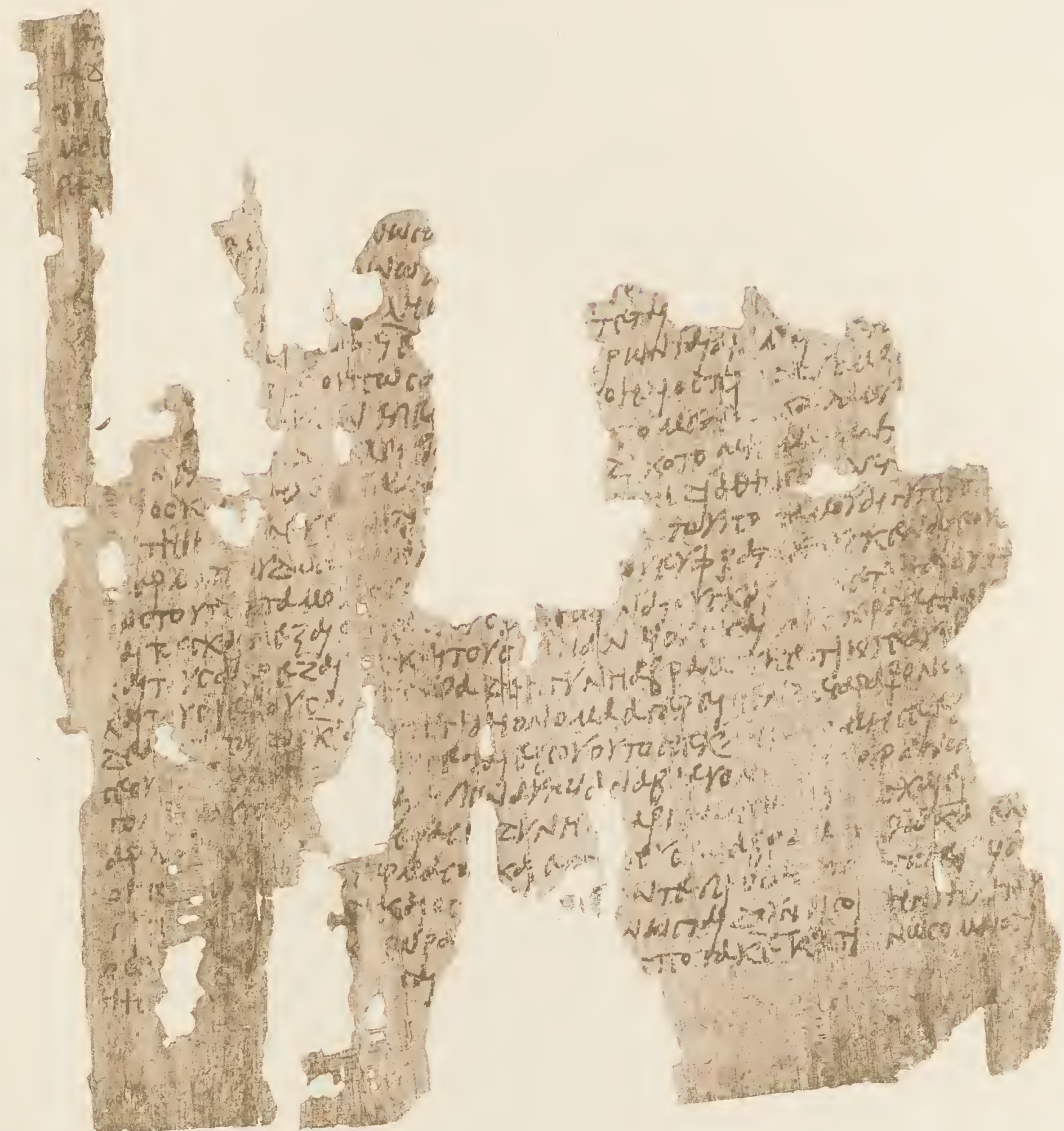




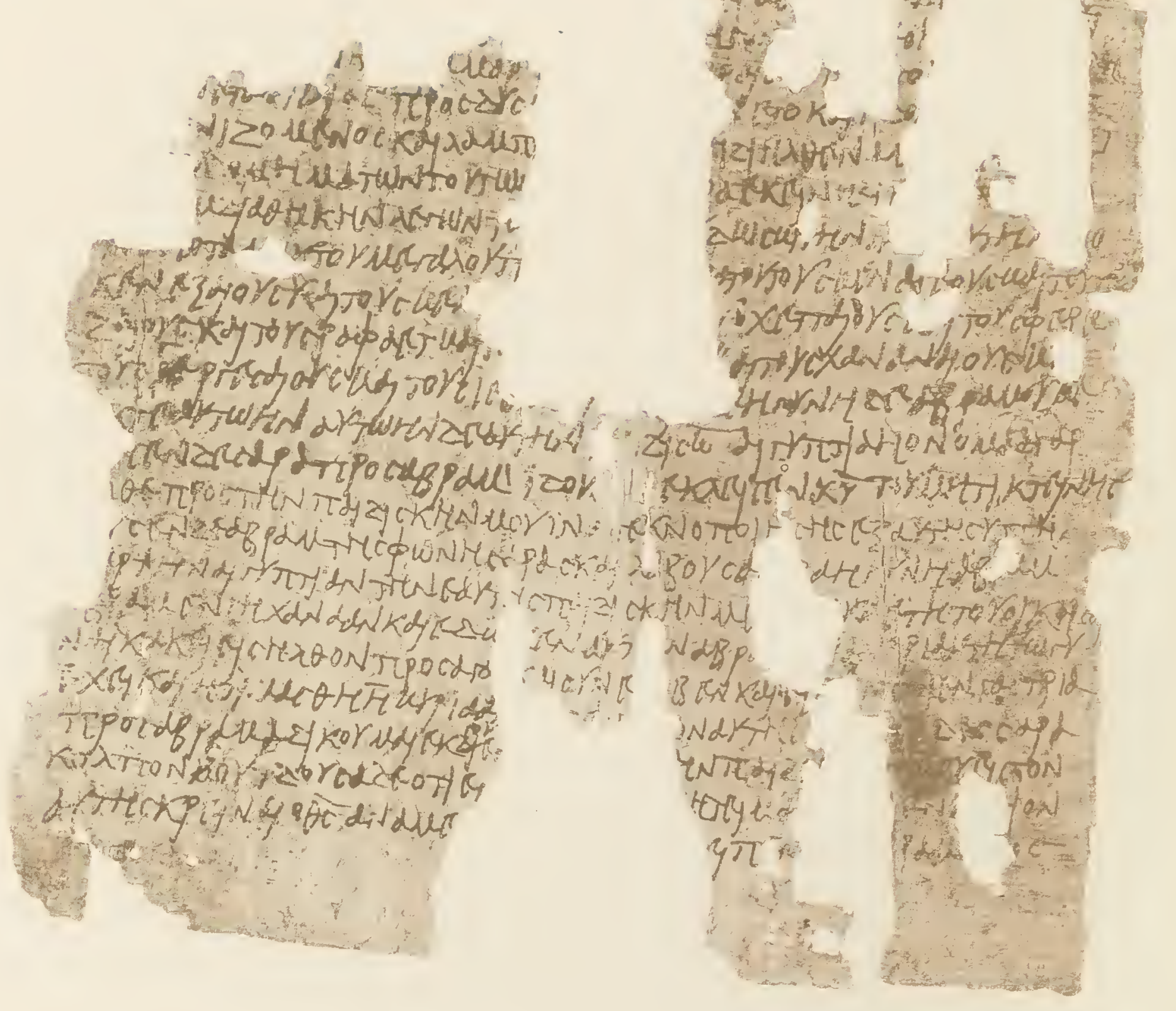




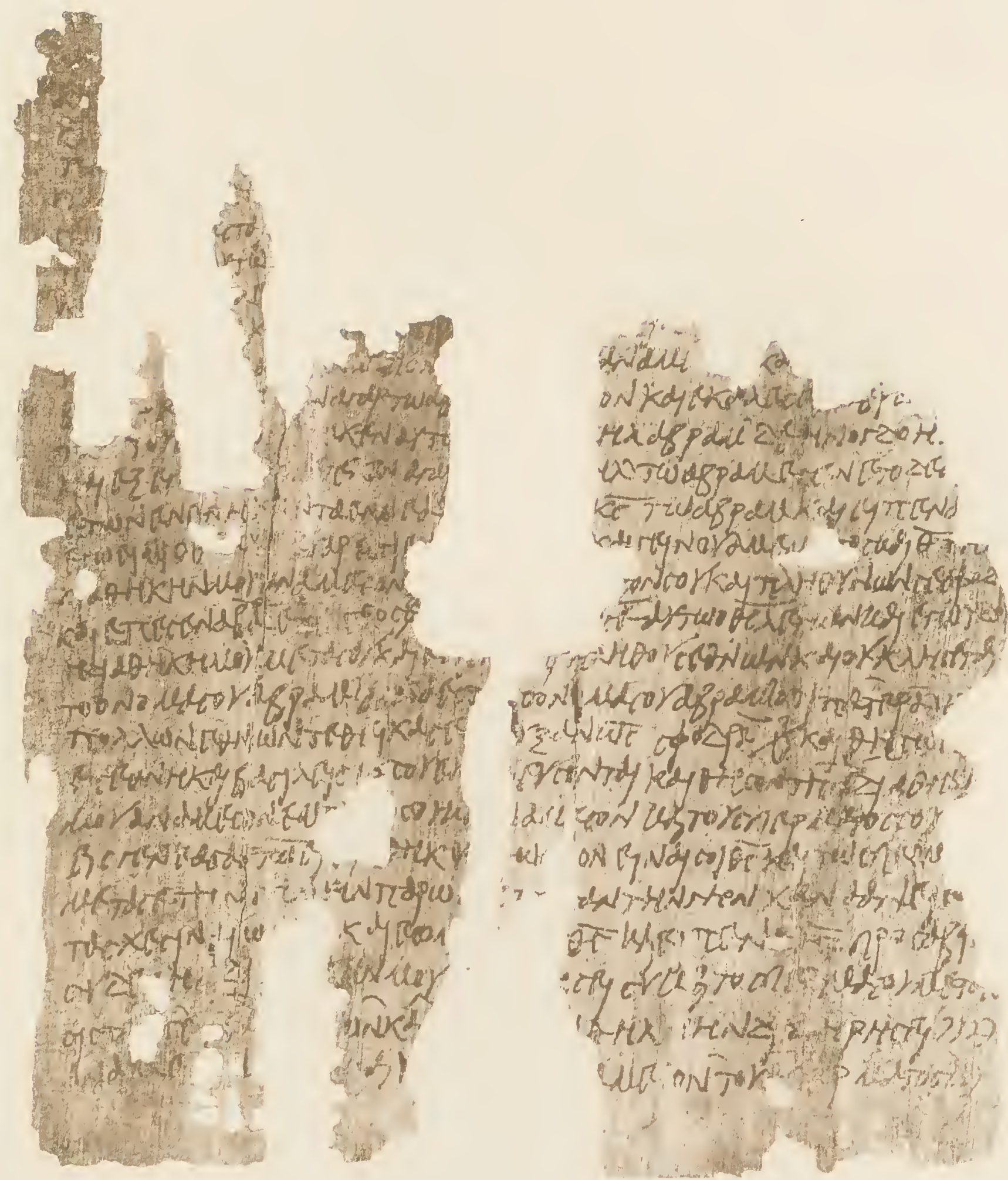



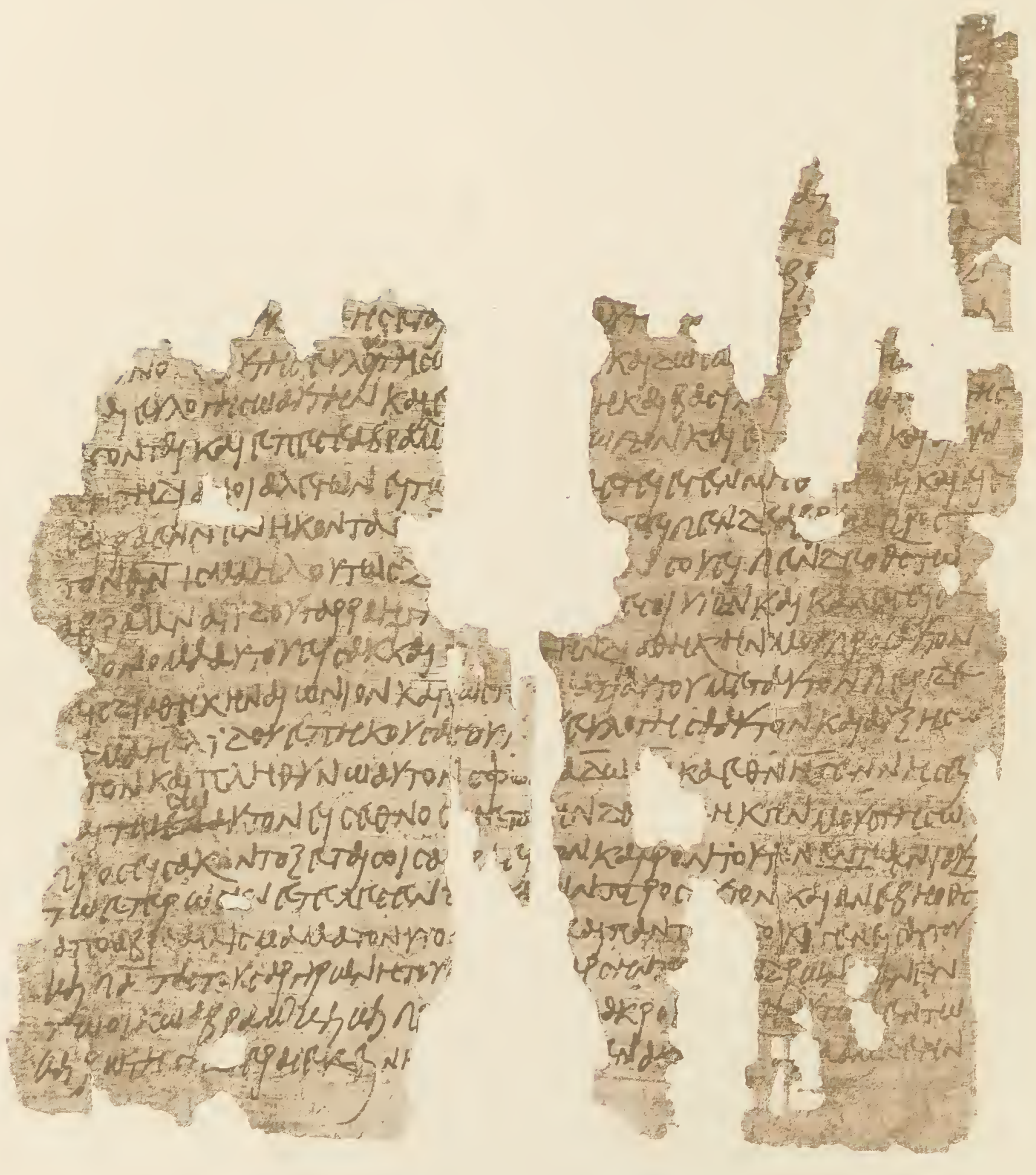

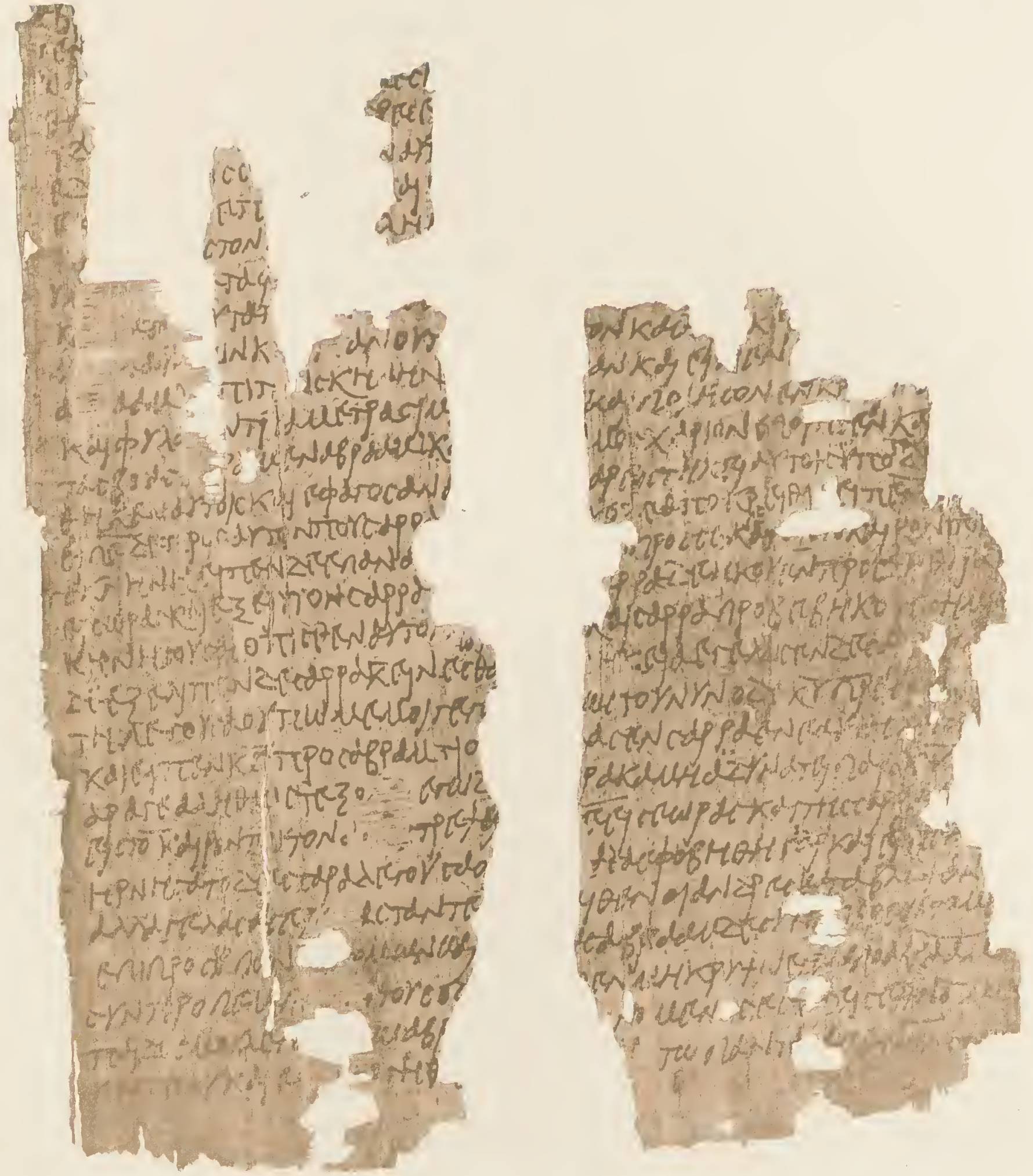


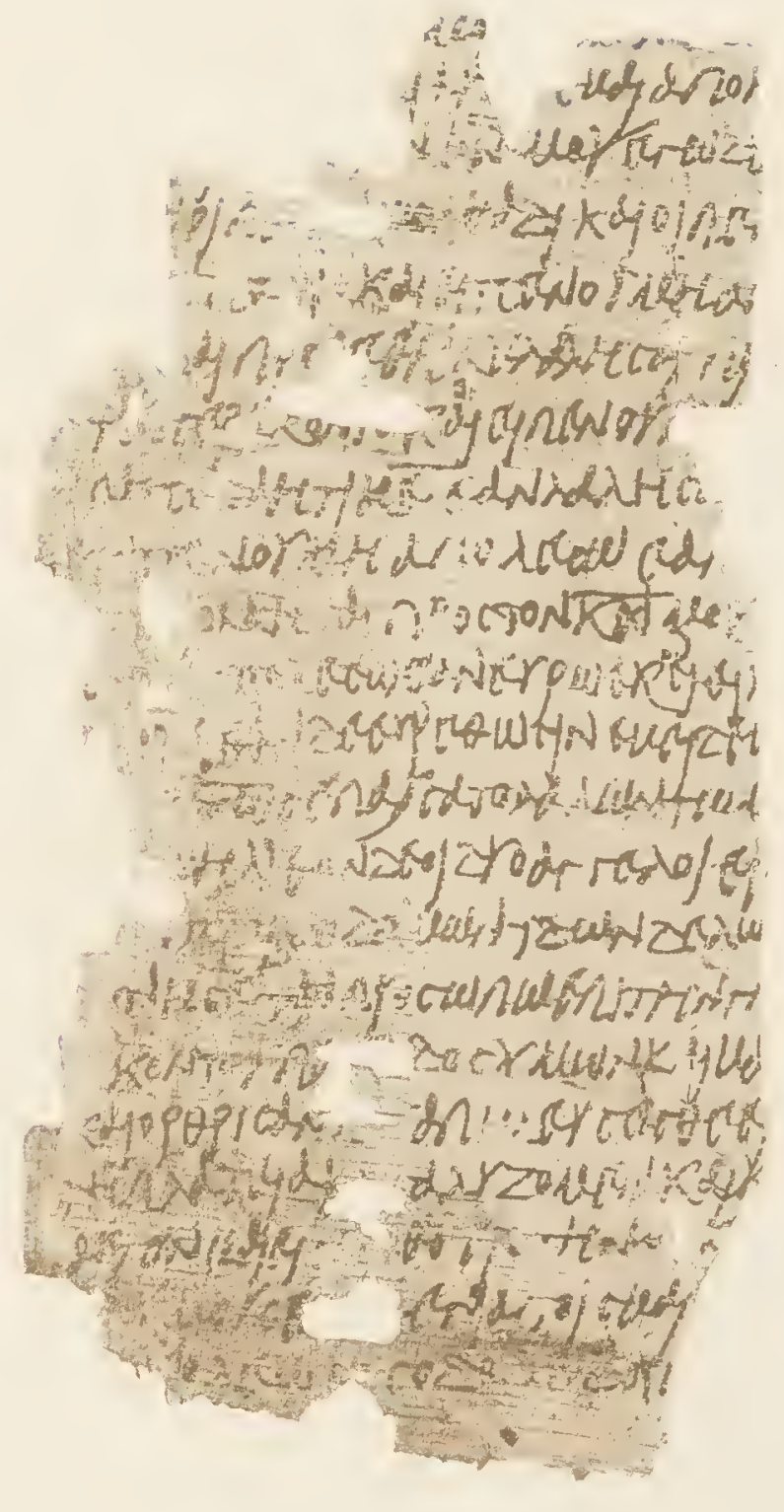

20
$4 \times 3$
4

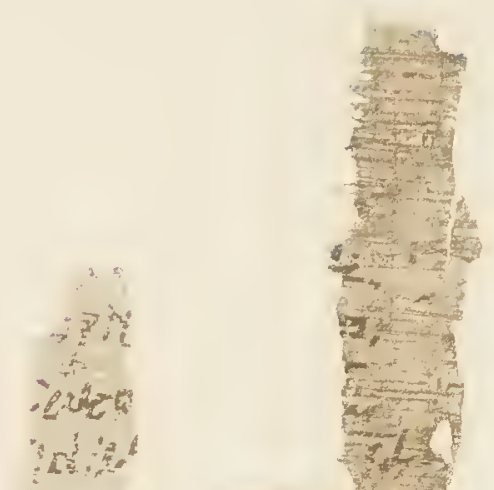

ofsety

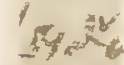

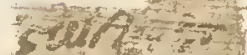

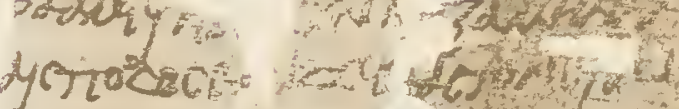

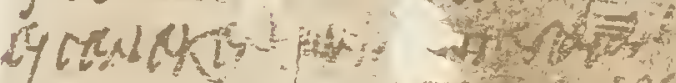

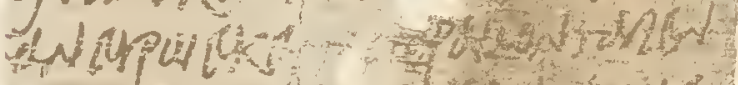
If

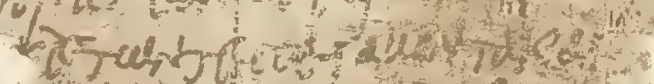

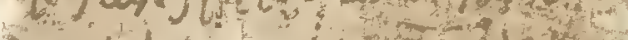

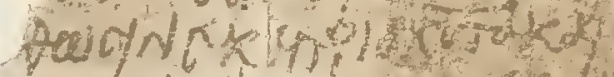

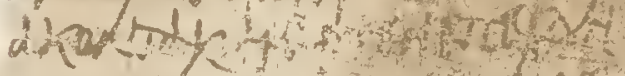

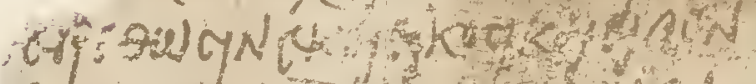

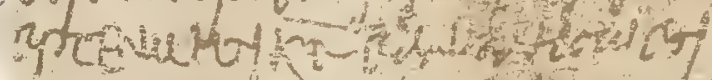

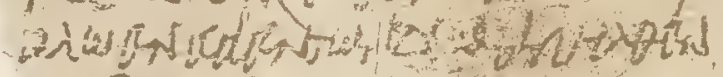

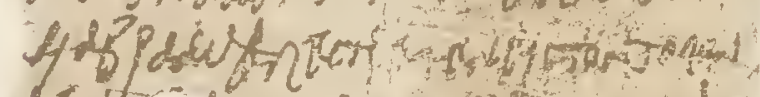

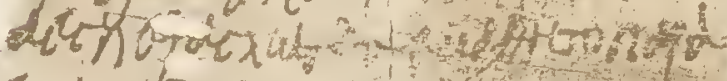

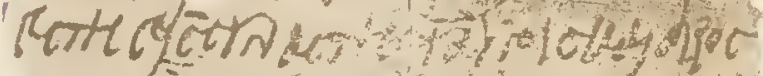

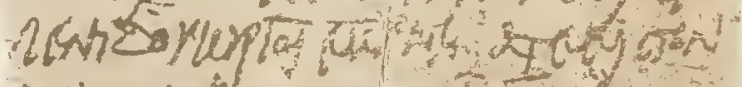

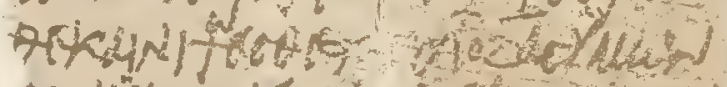

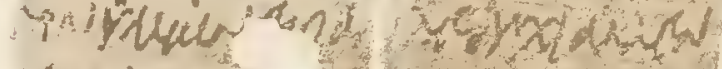
'To dy

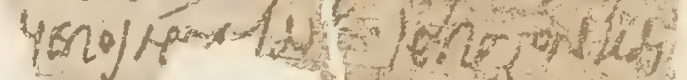

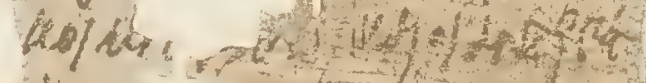

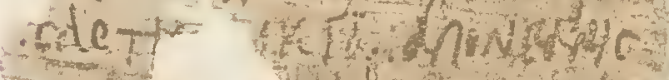

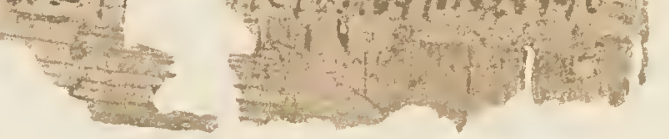




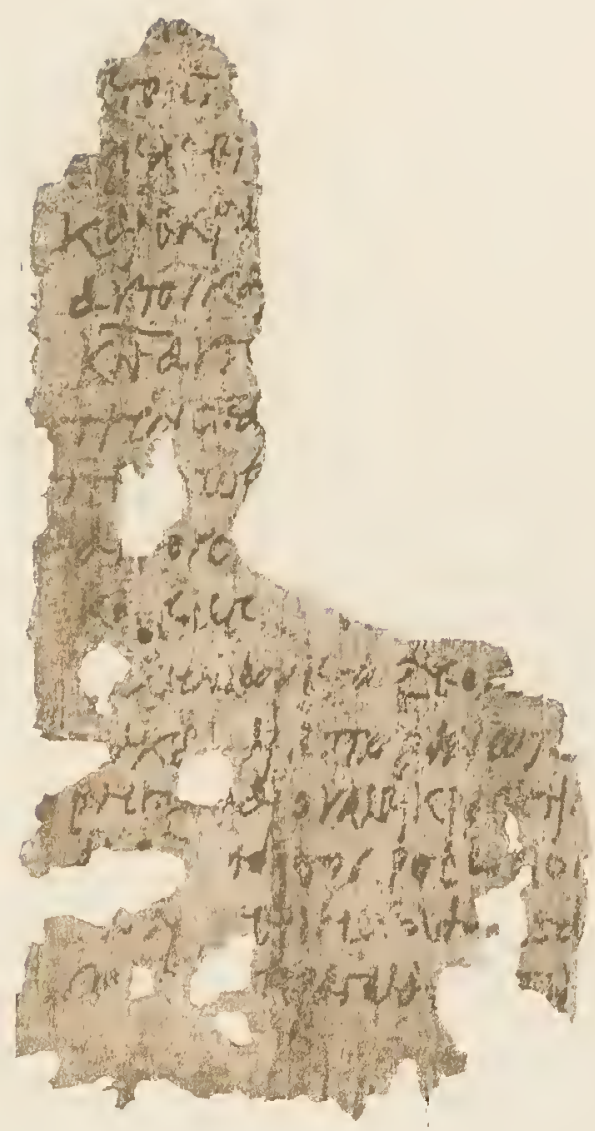




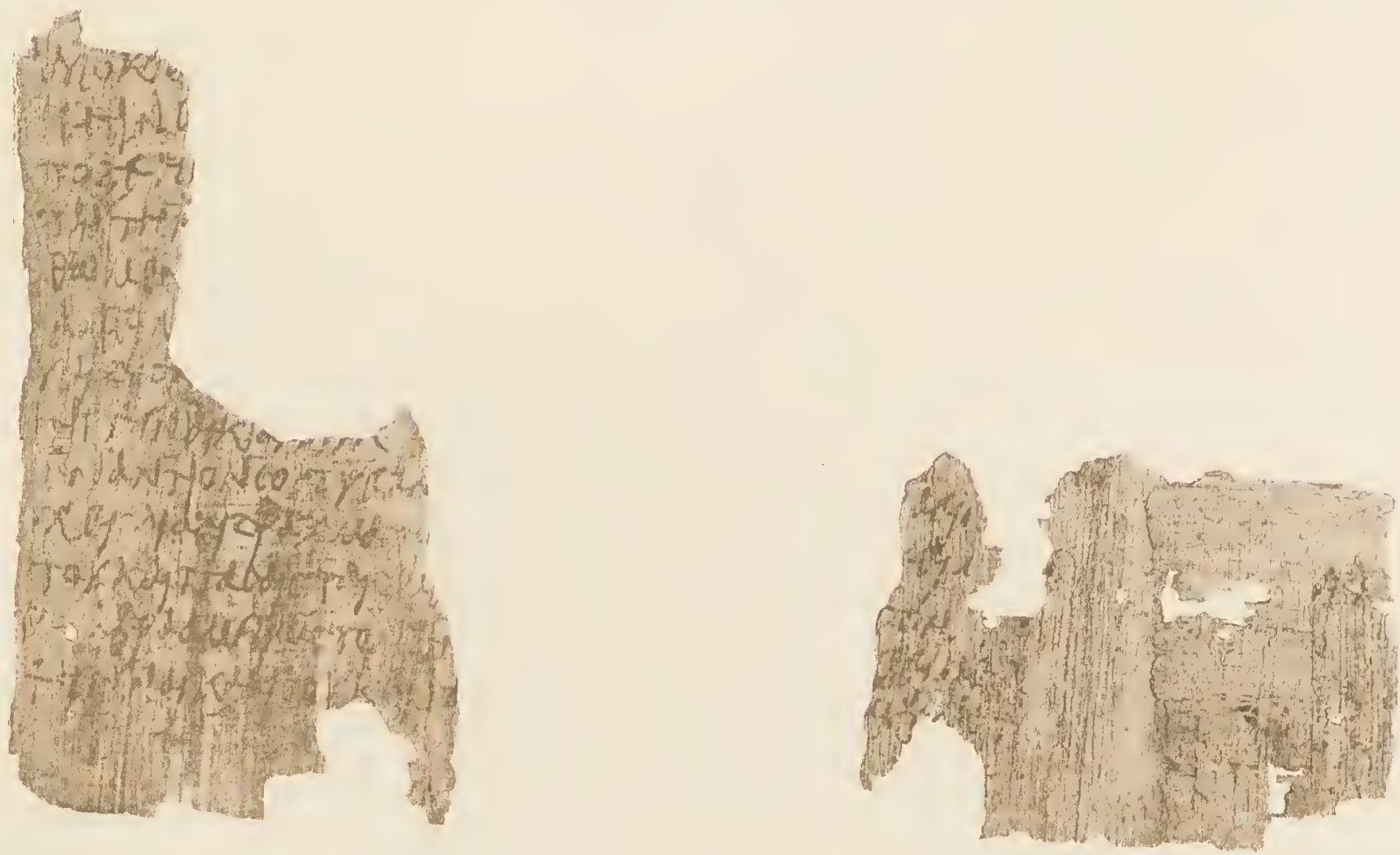

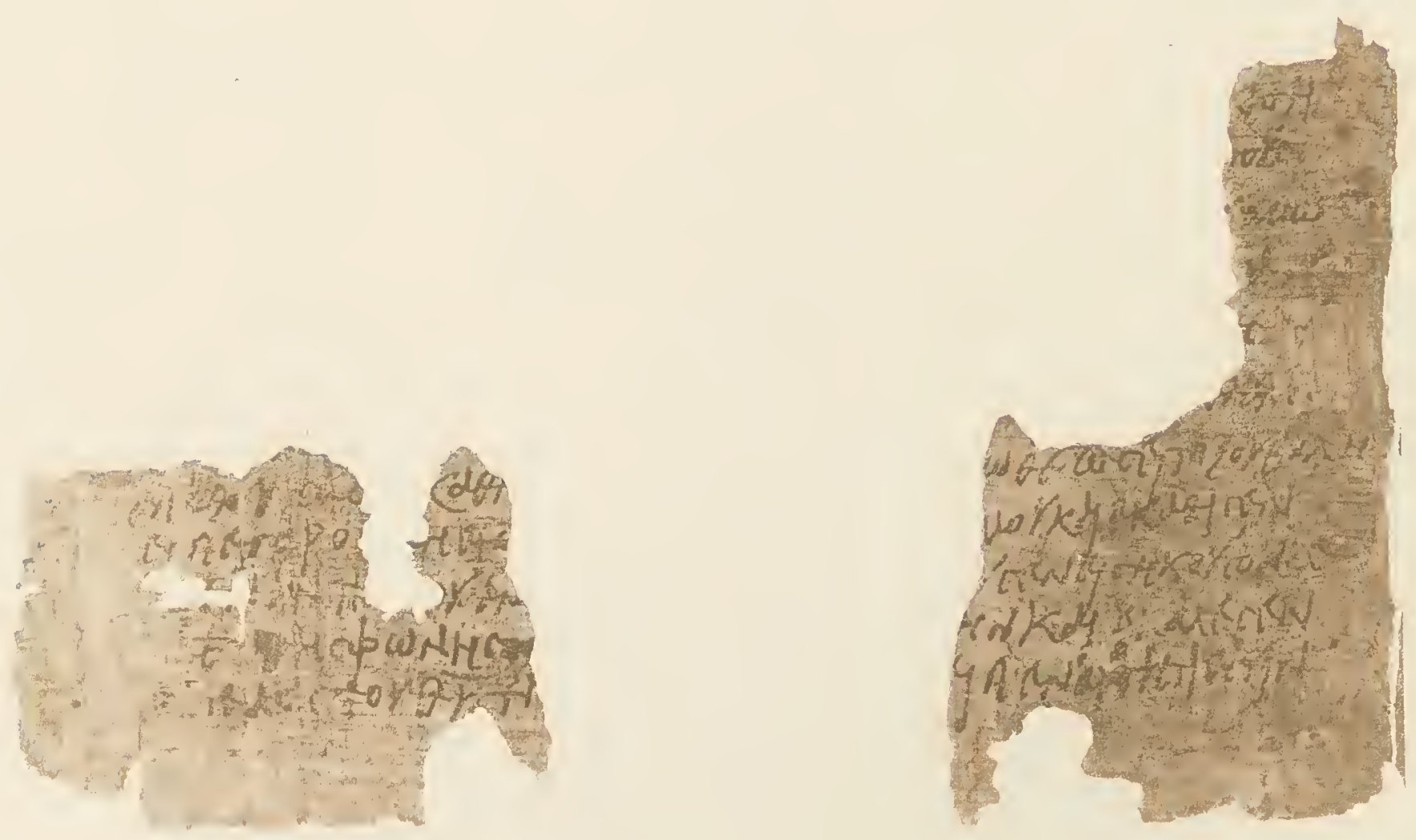

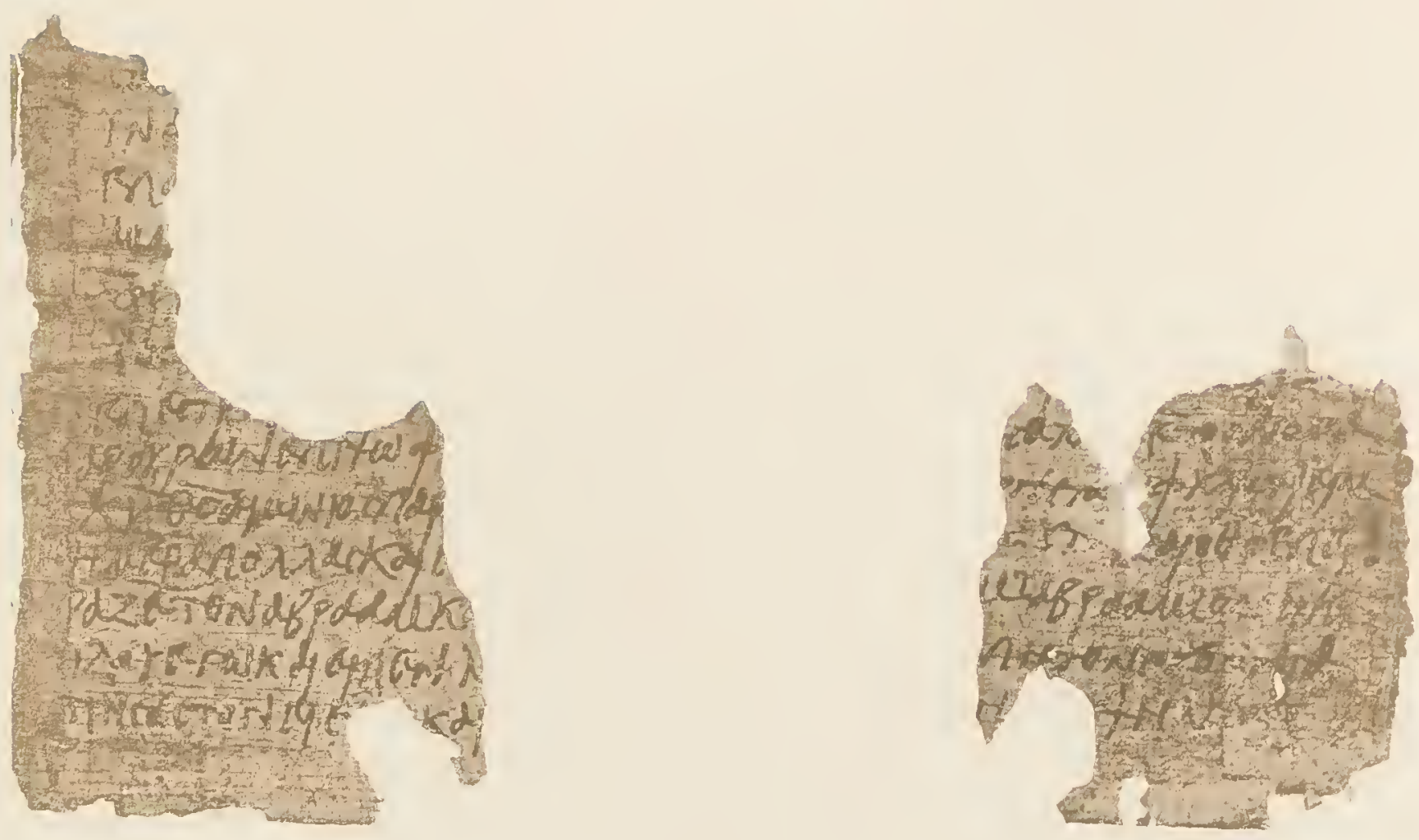

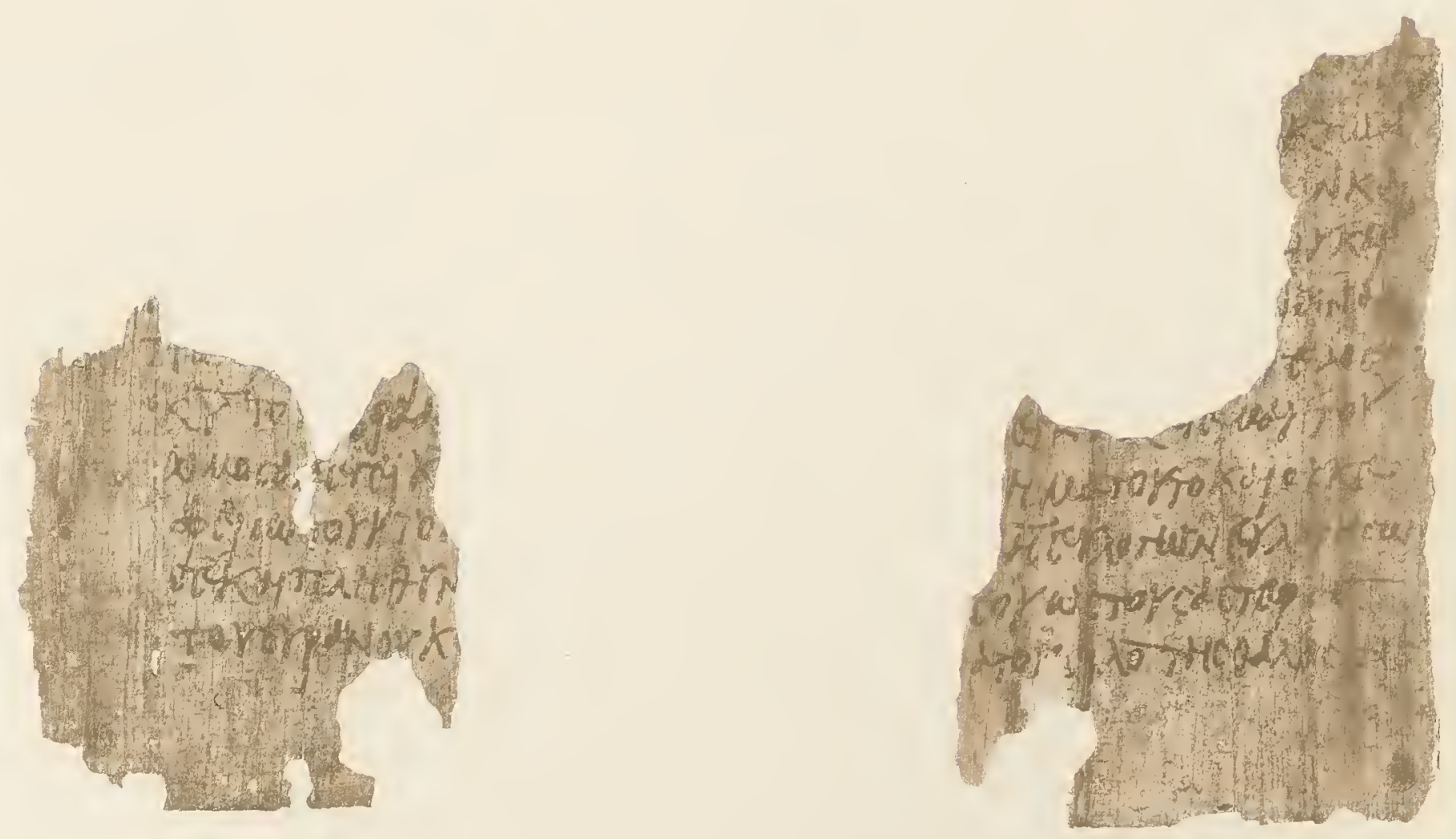

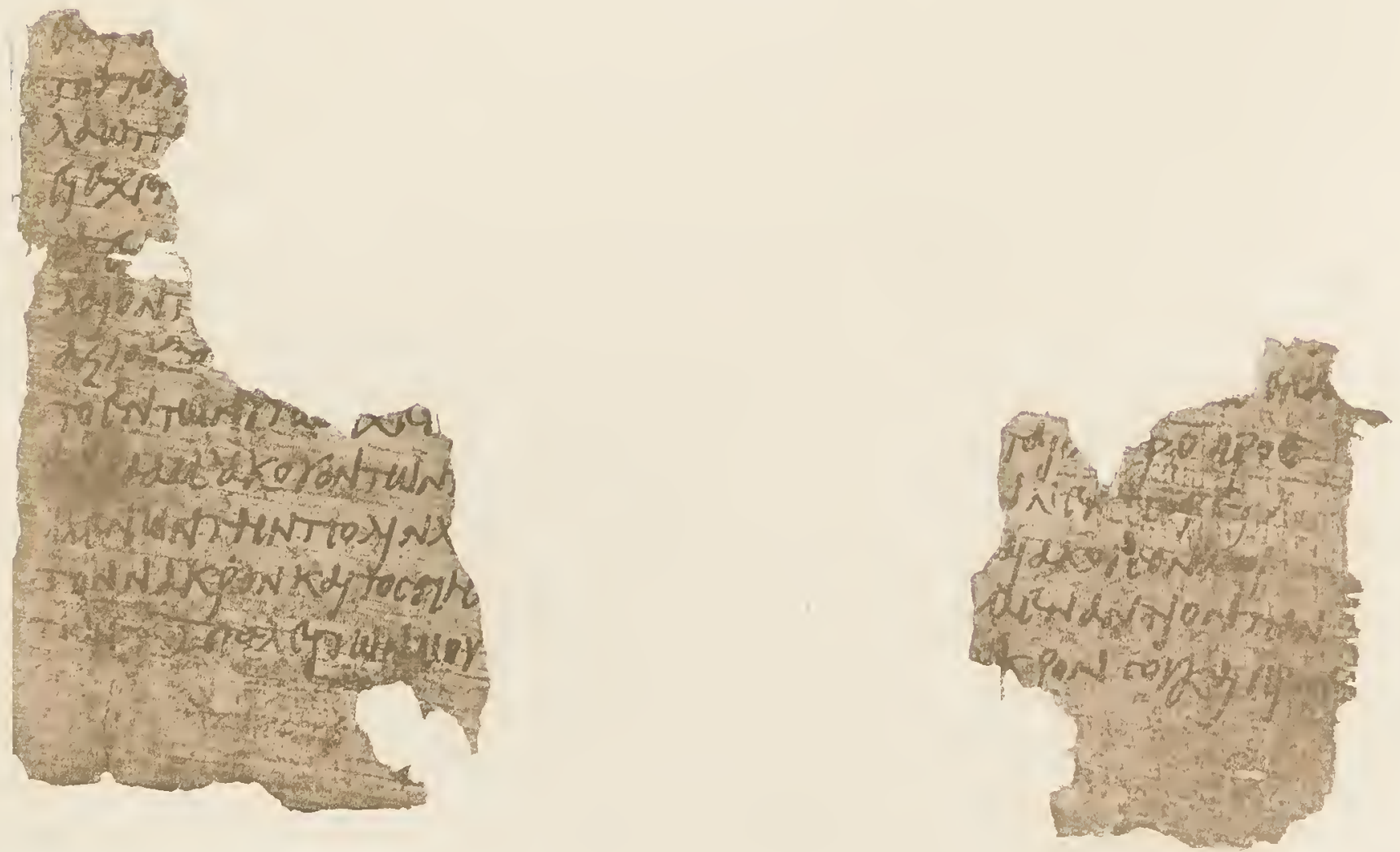

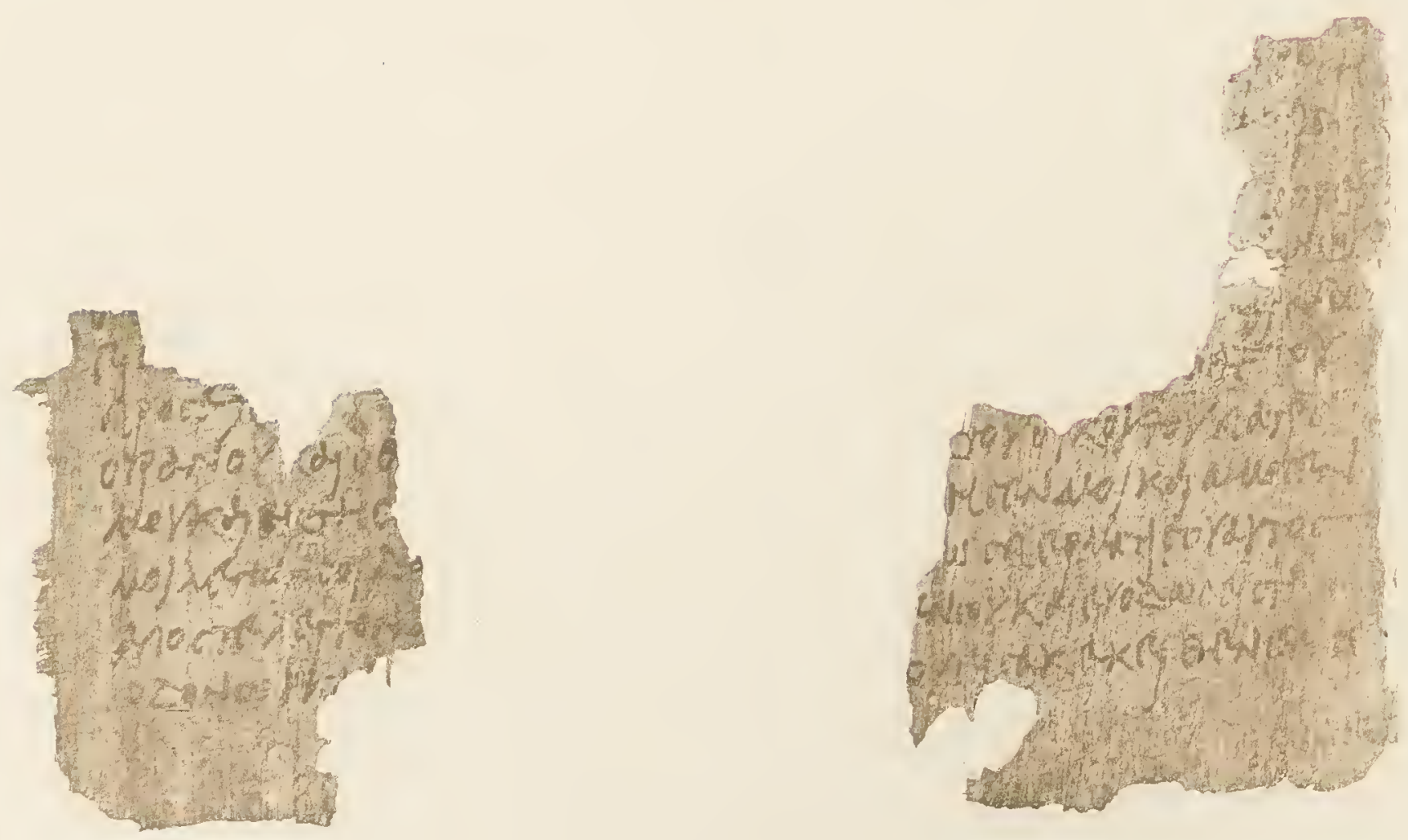

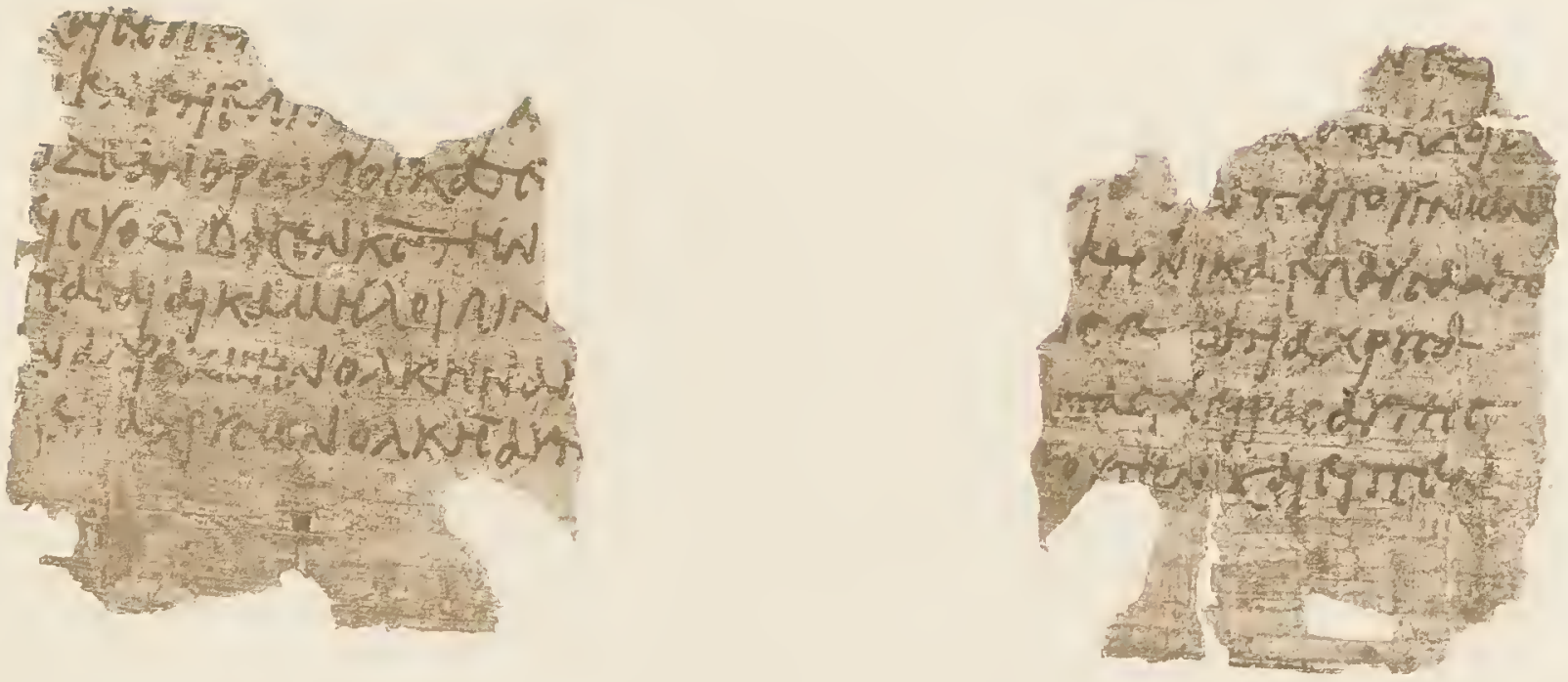

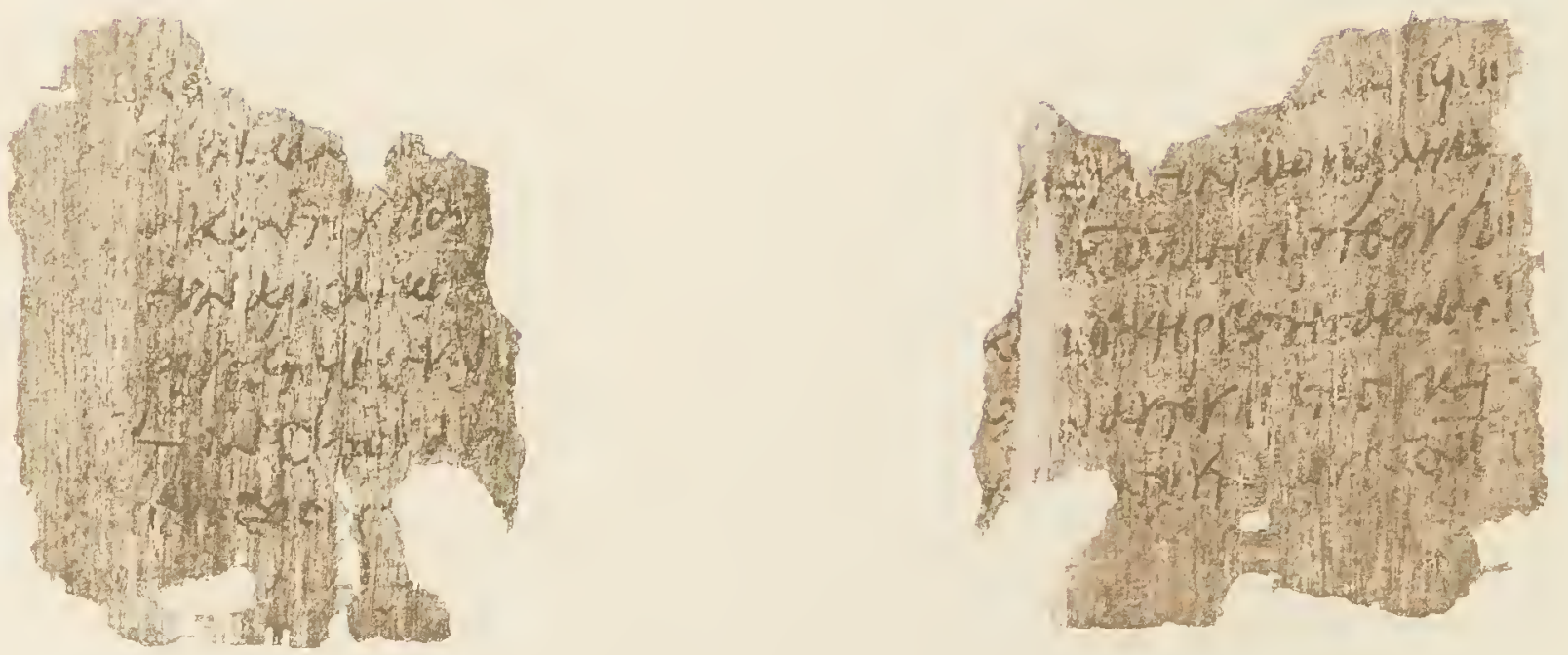

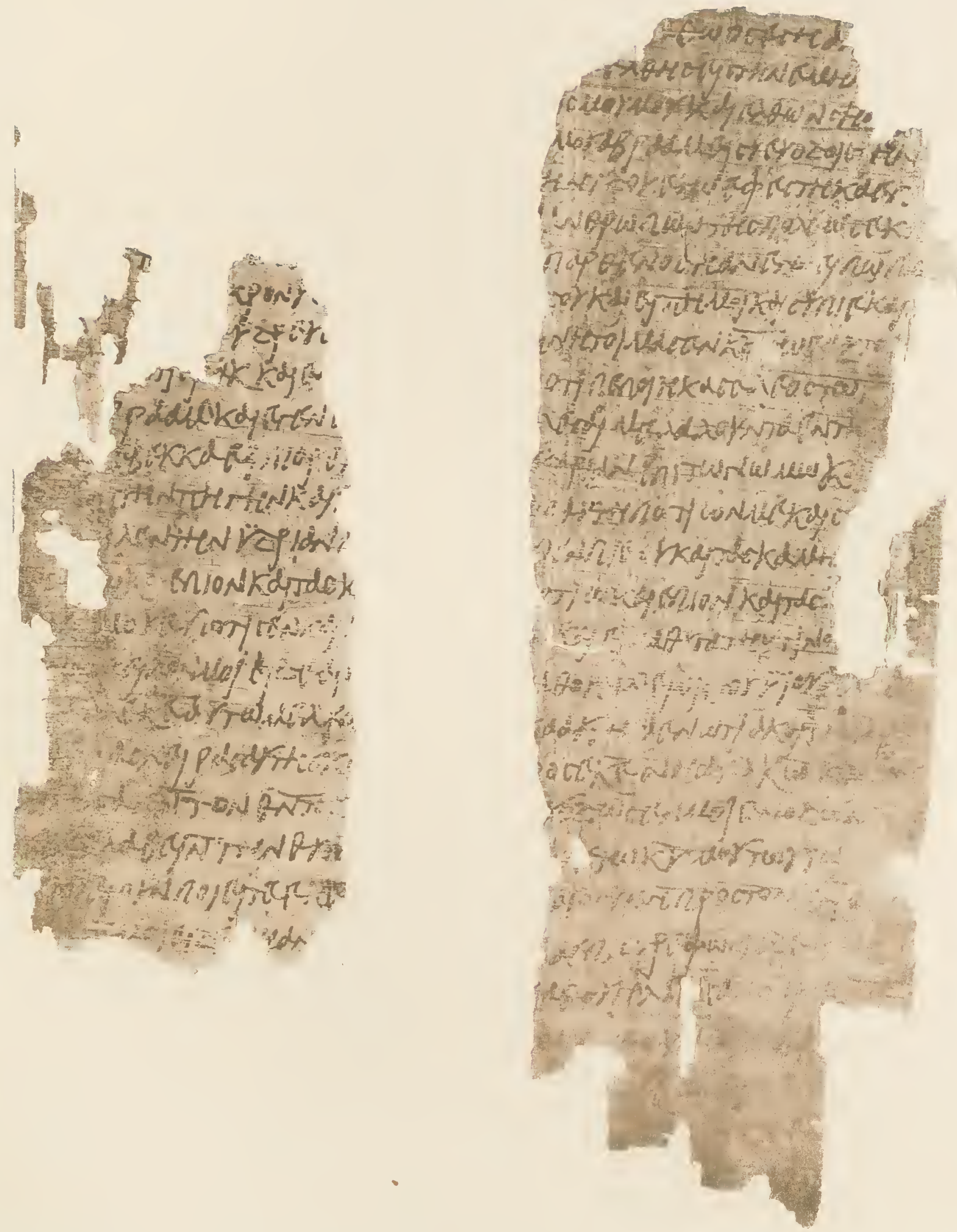

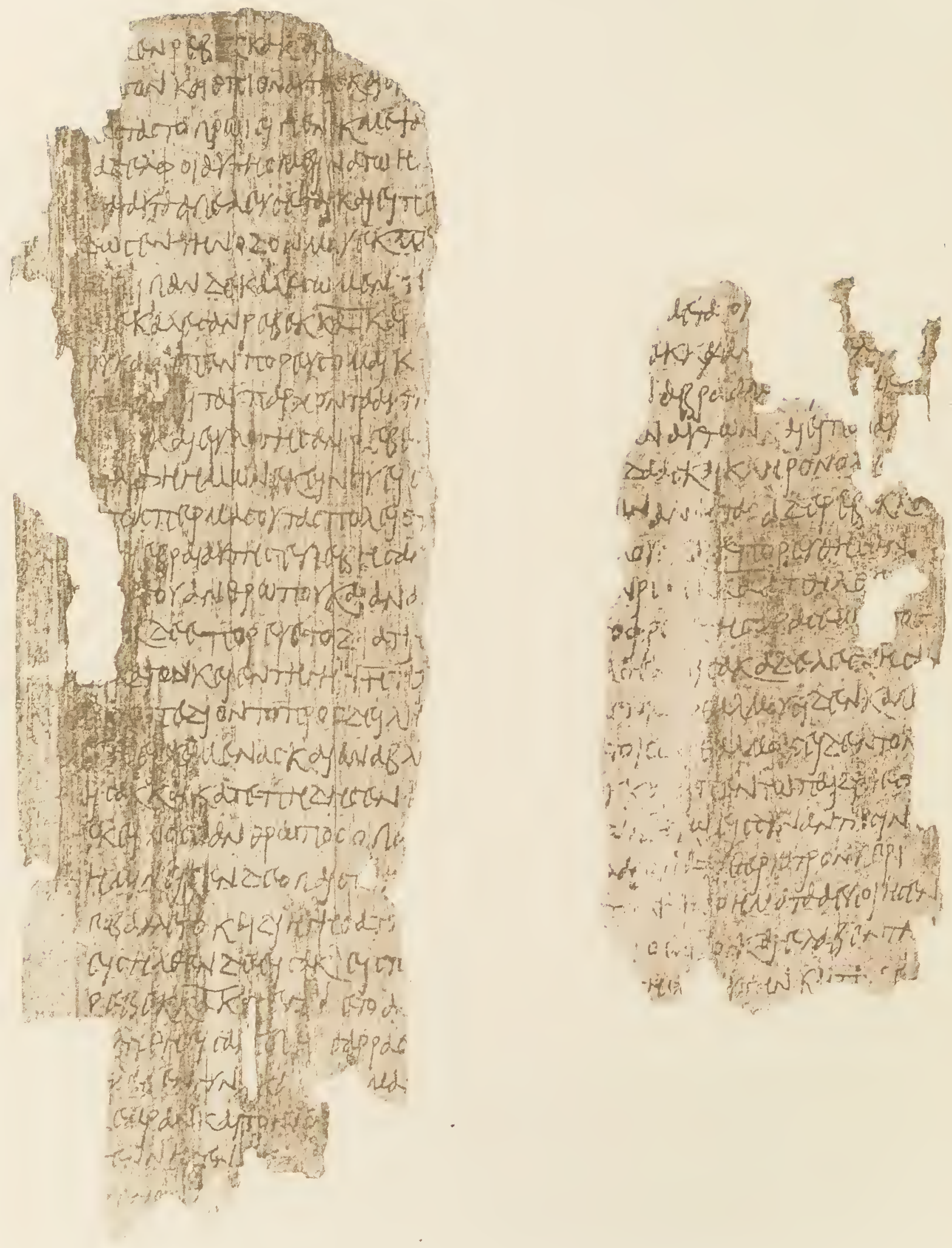


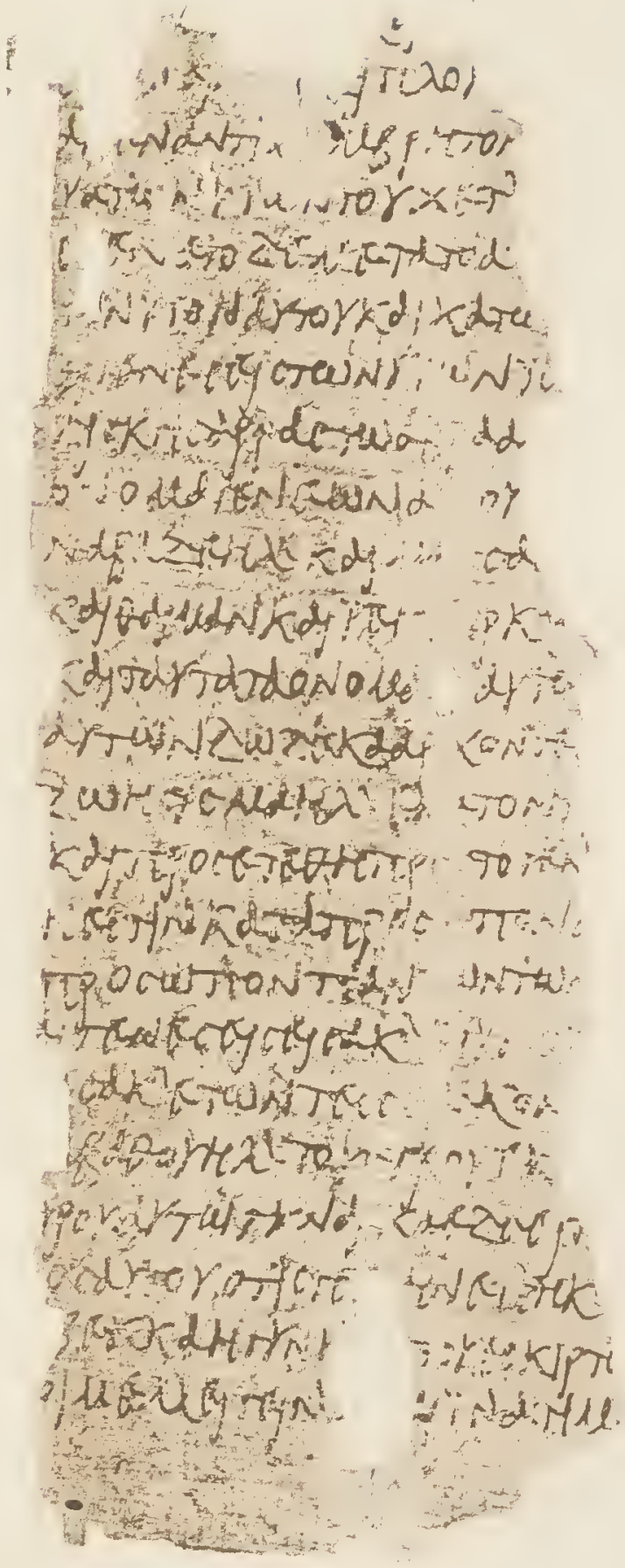



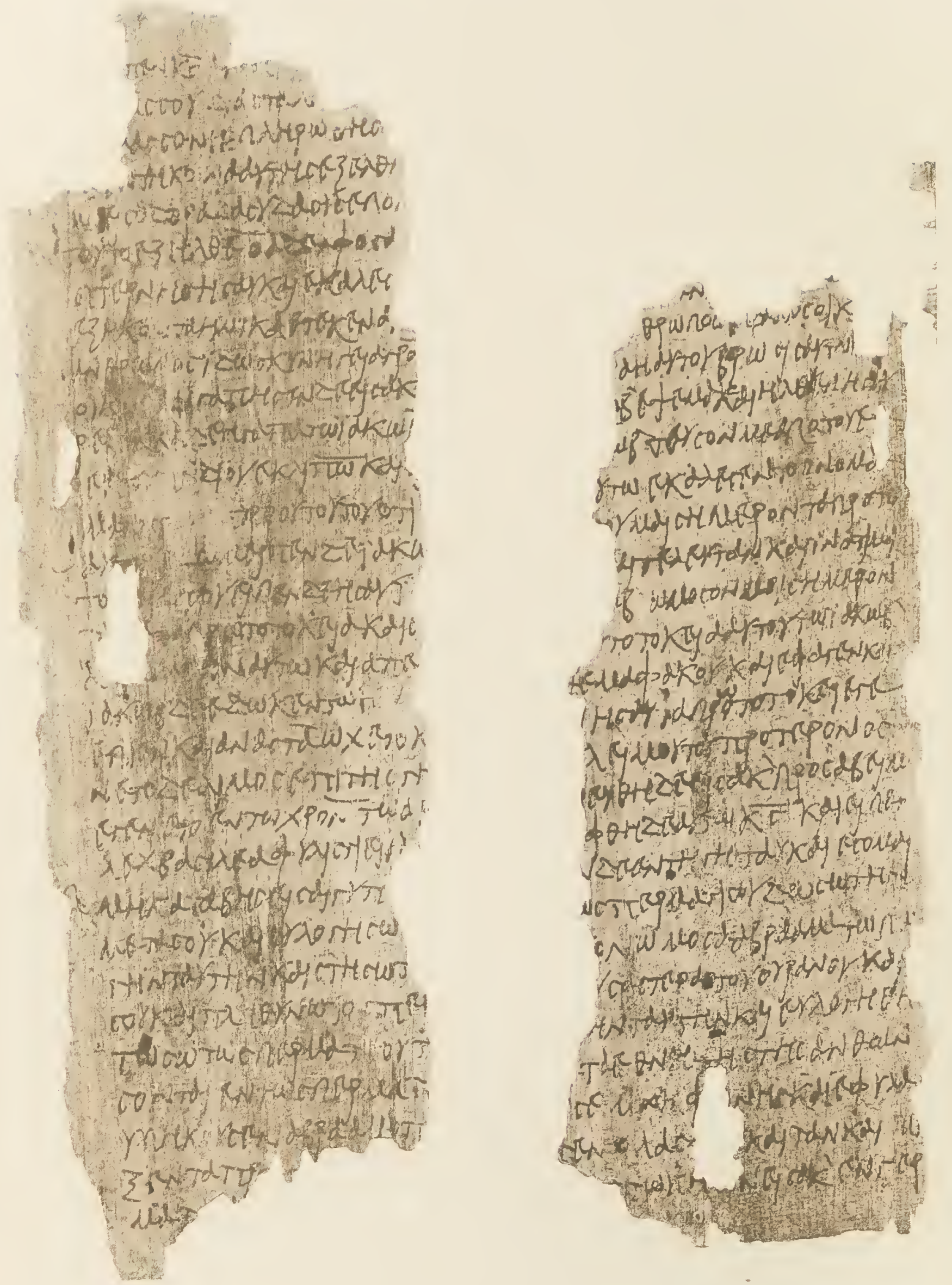

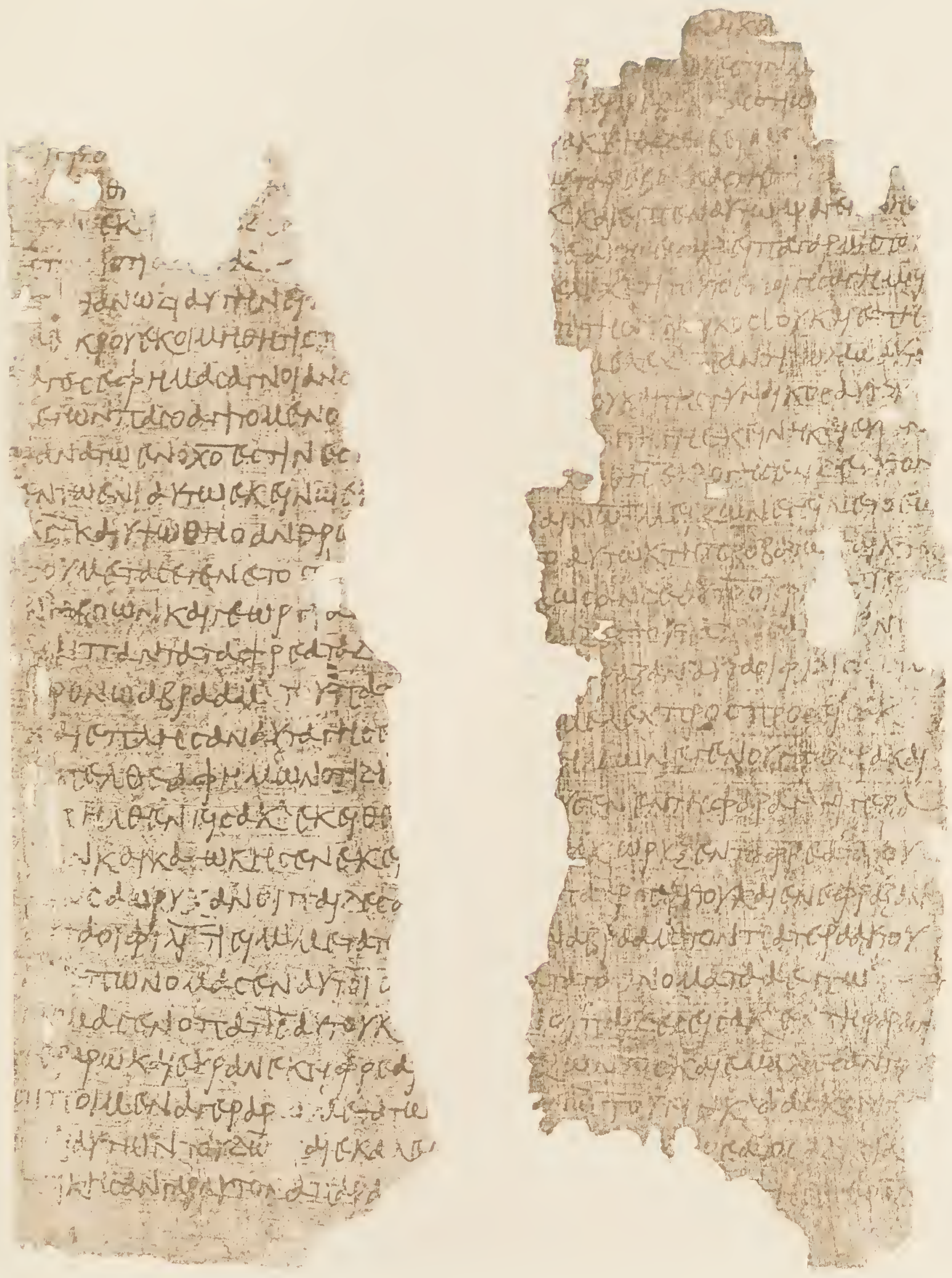

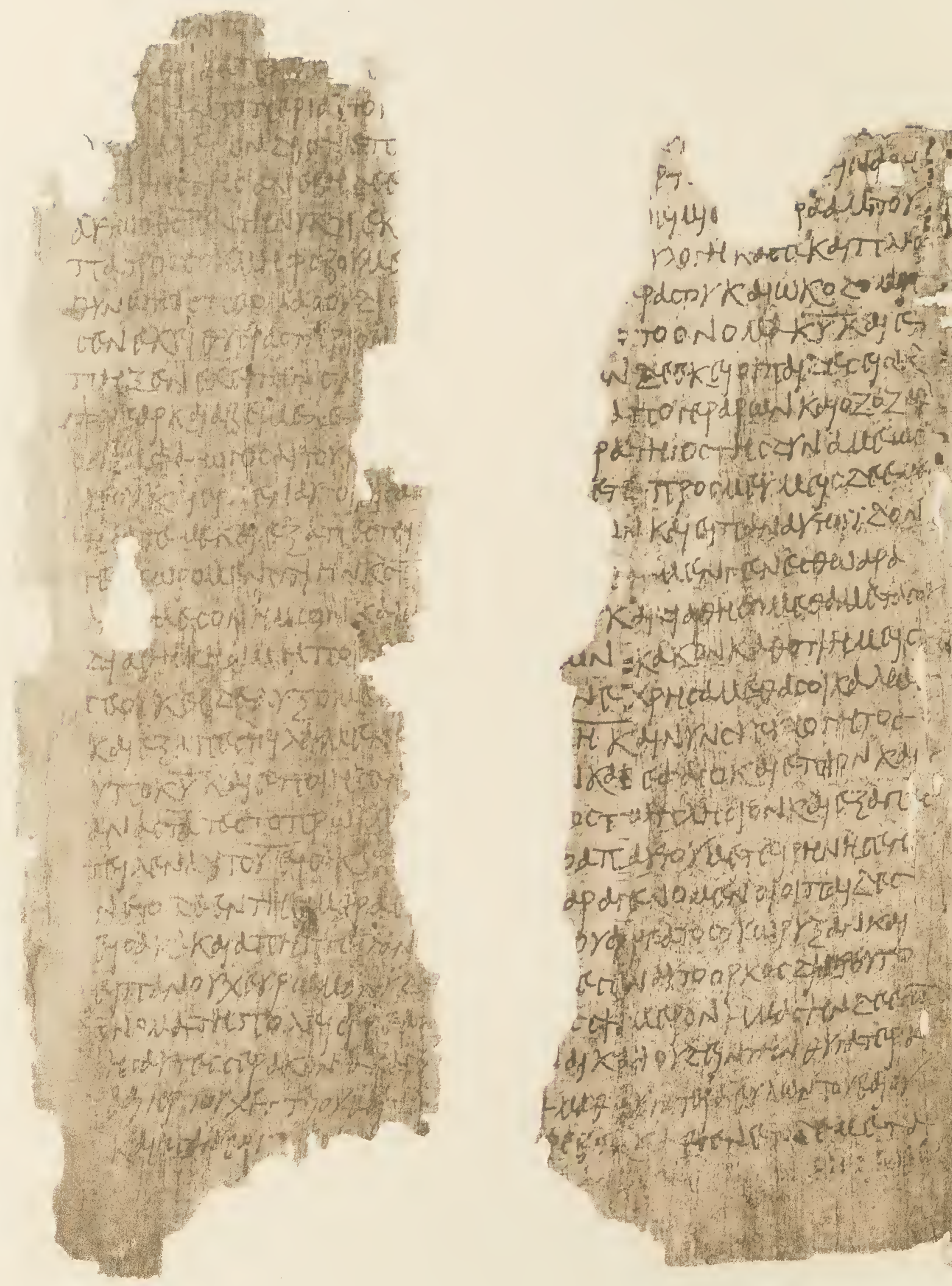

pdonk kauko ody

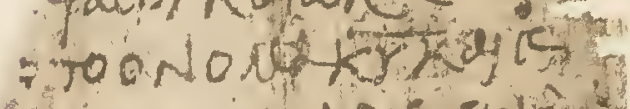

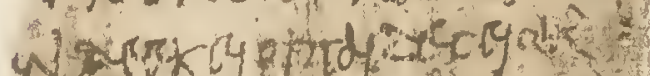

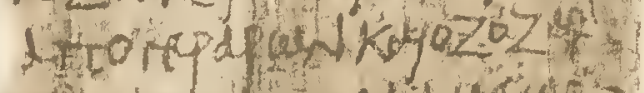

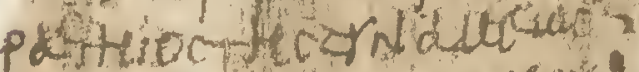

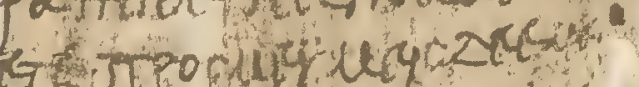

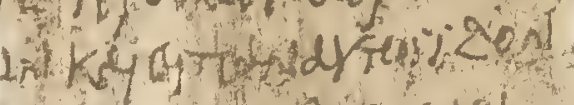
- dín racetouded

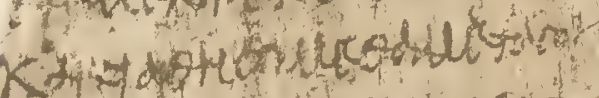

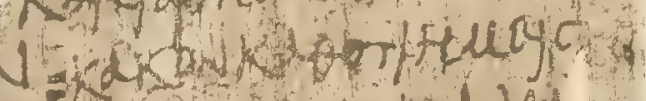

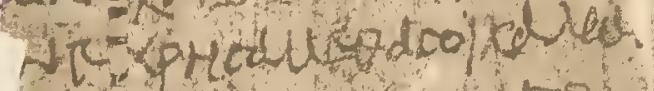
HXHNindrolomac

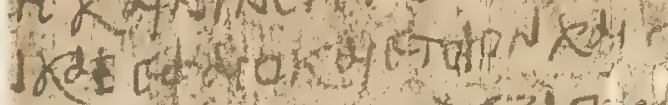

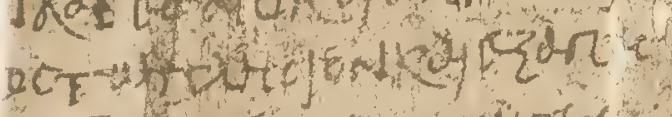

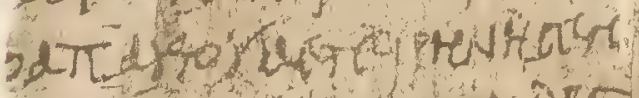

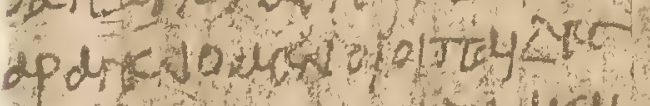

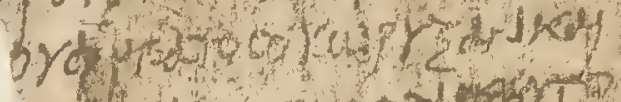

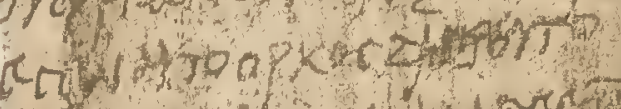

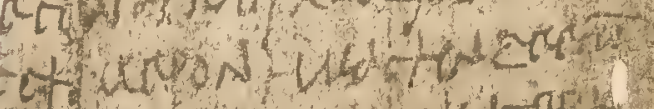

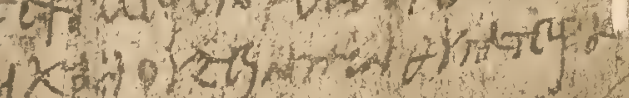

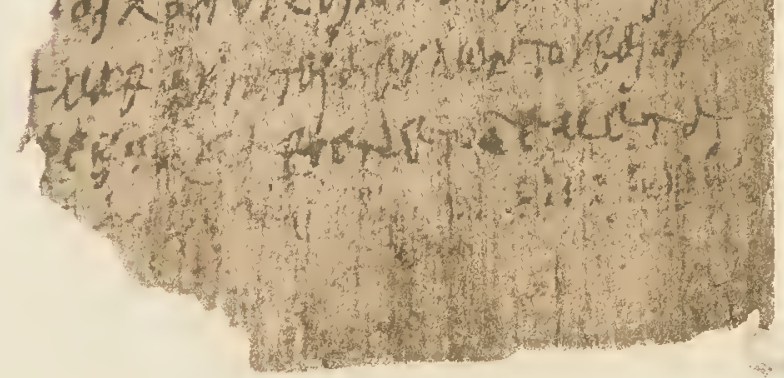



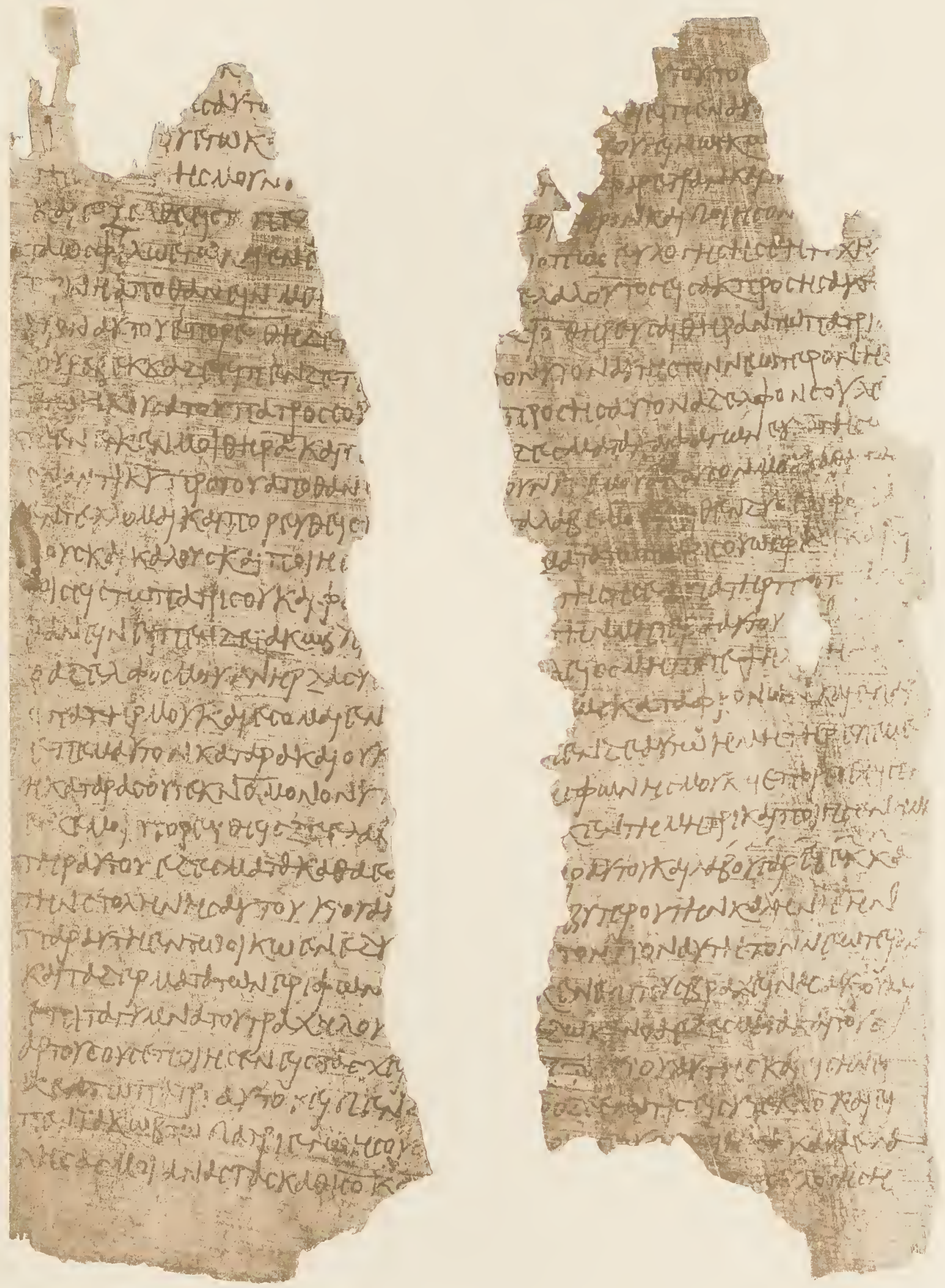

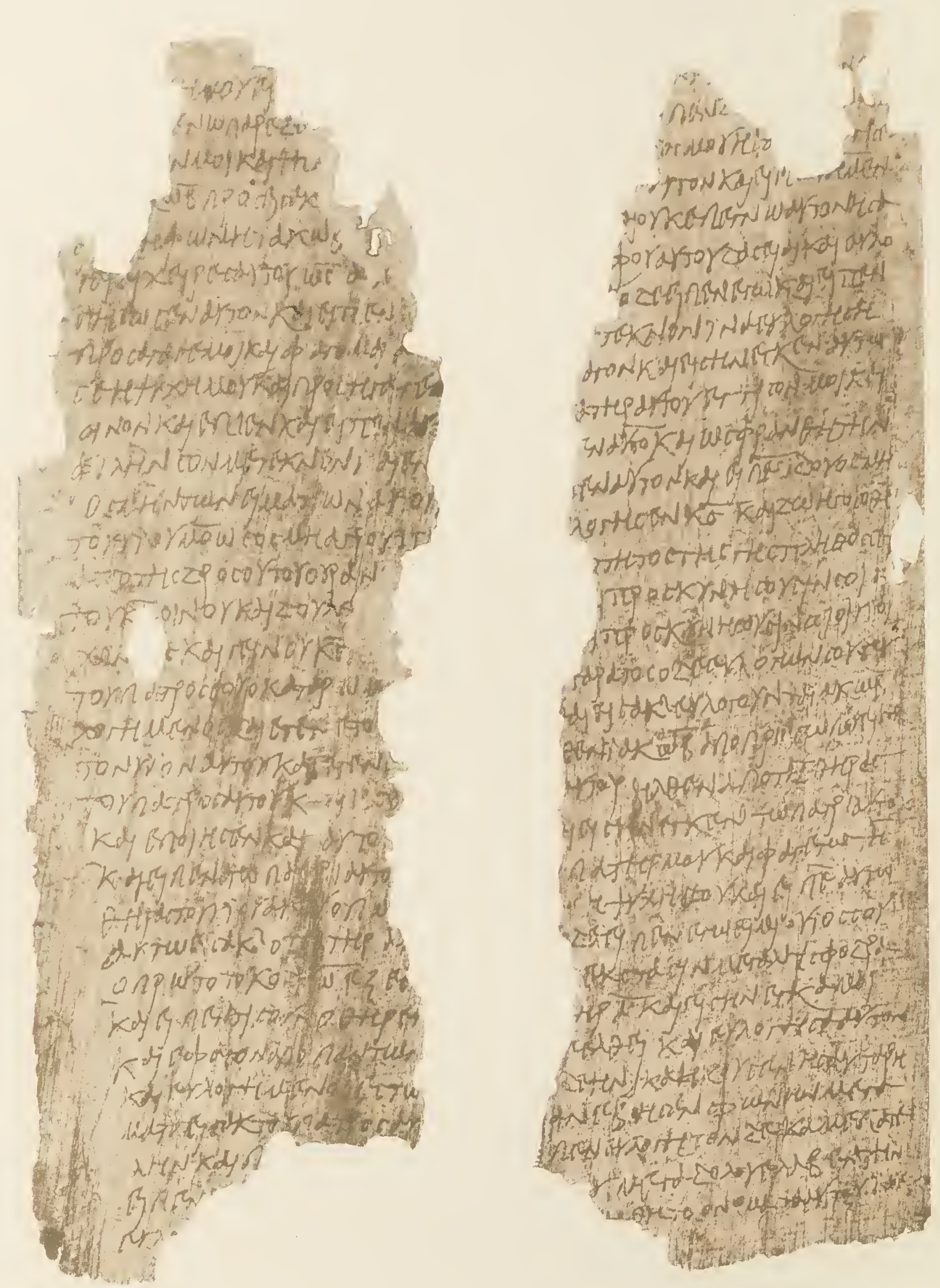


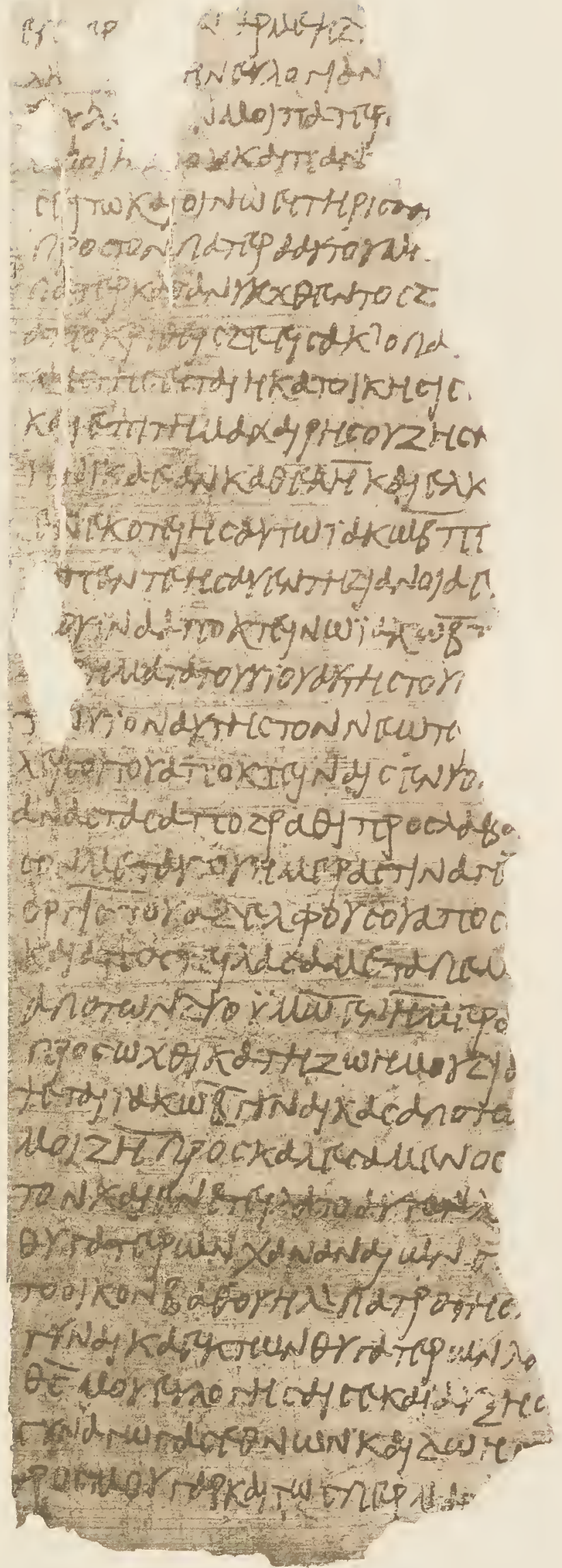



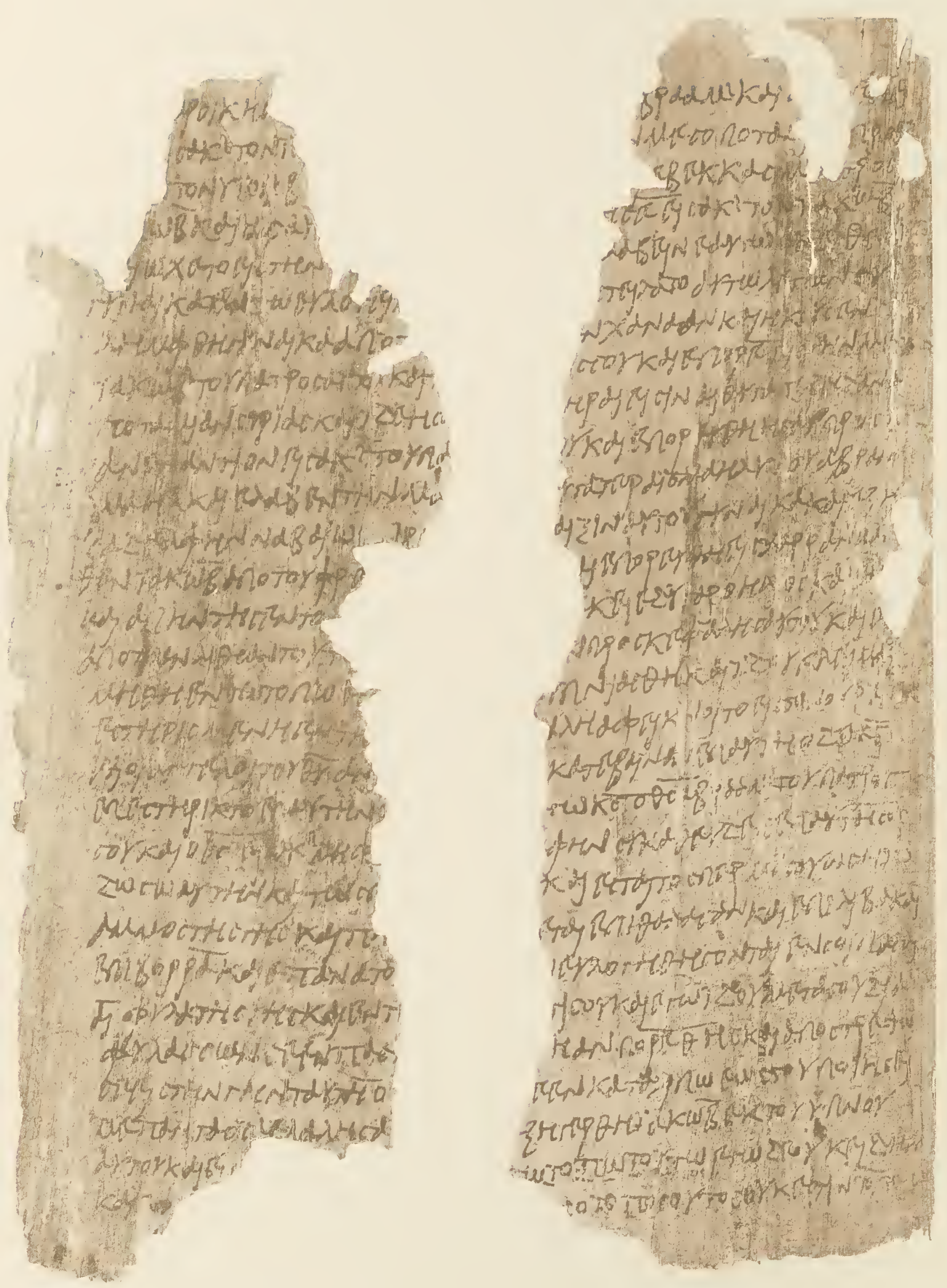


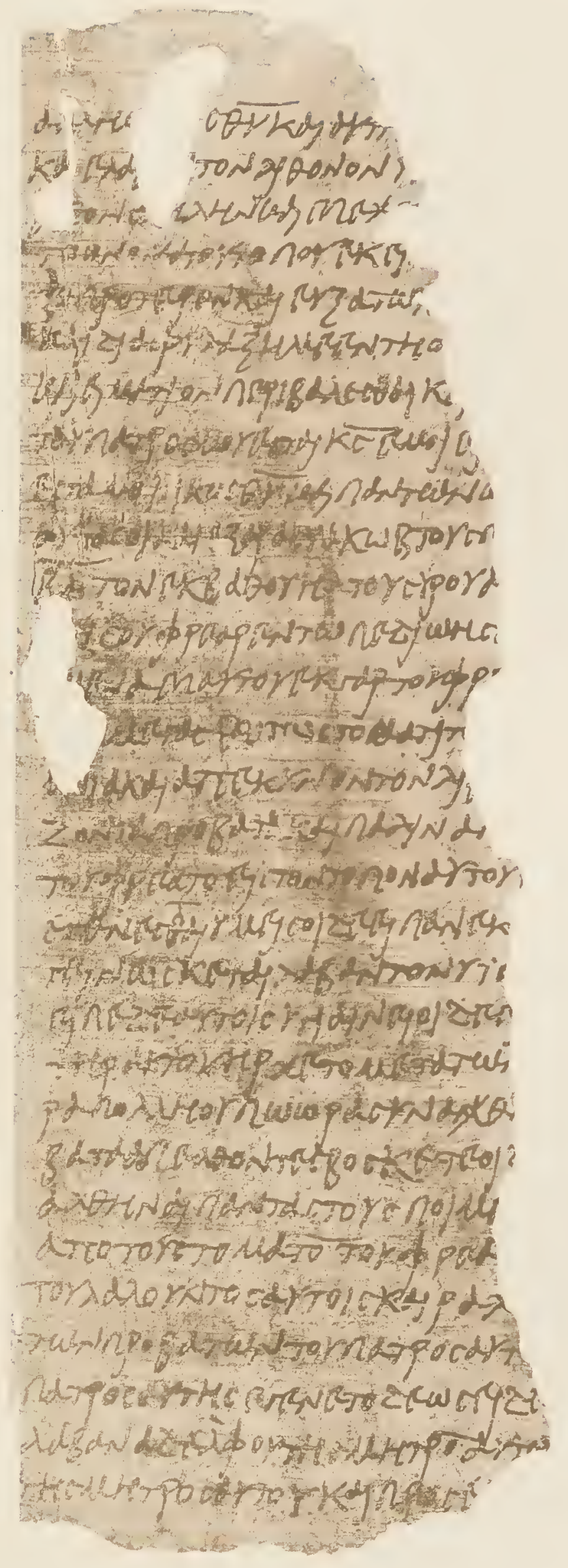

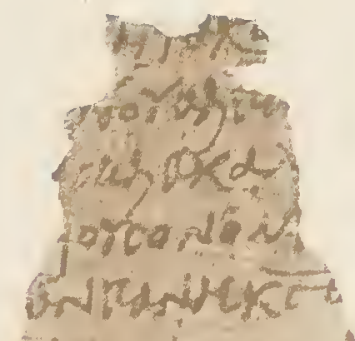

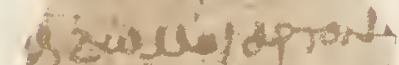

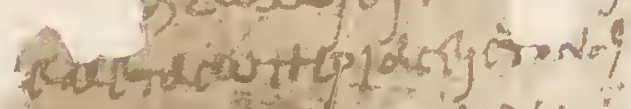

argeonfsite

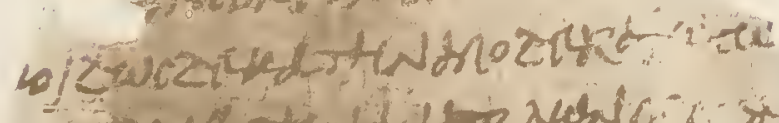

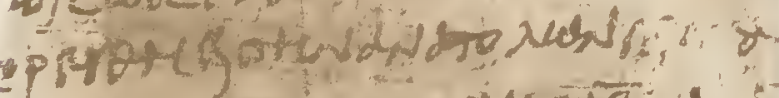

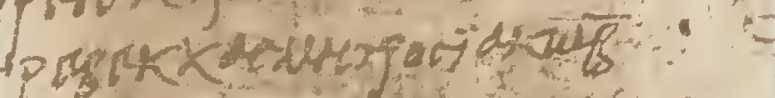
y.

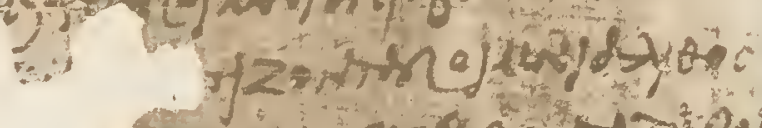

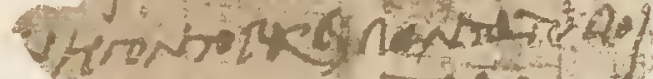

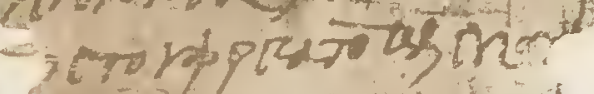
To" rolcidkus intepo/ino

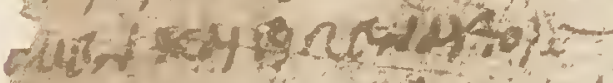

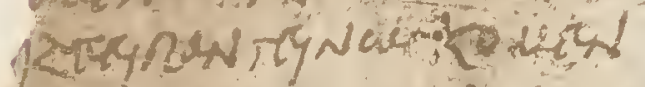

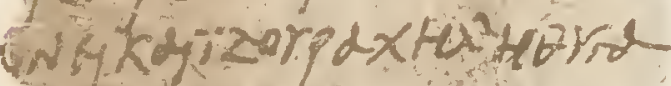

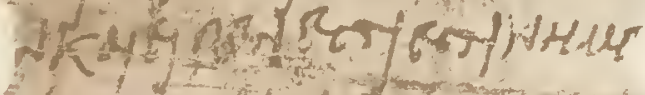

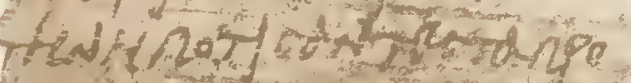

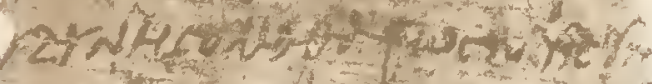

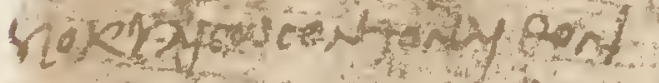

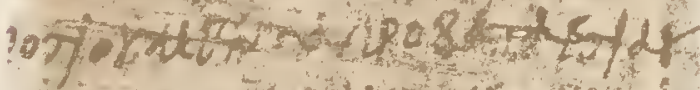

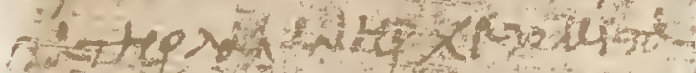

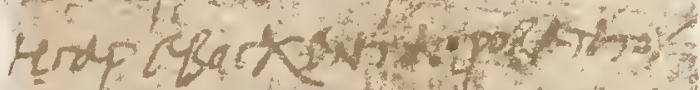

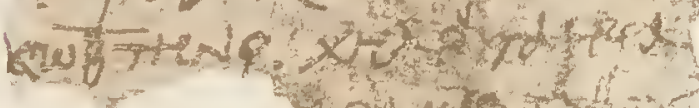

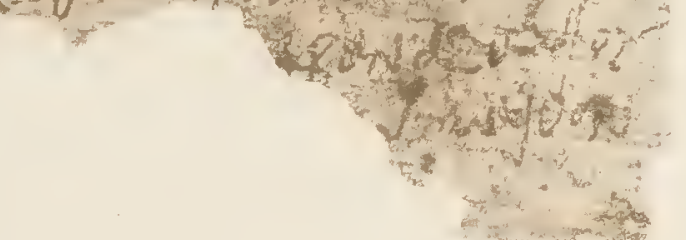



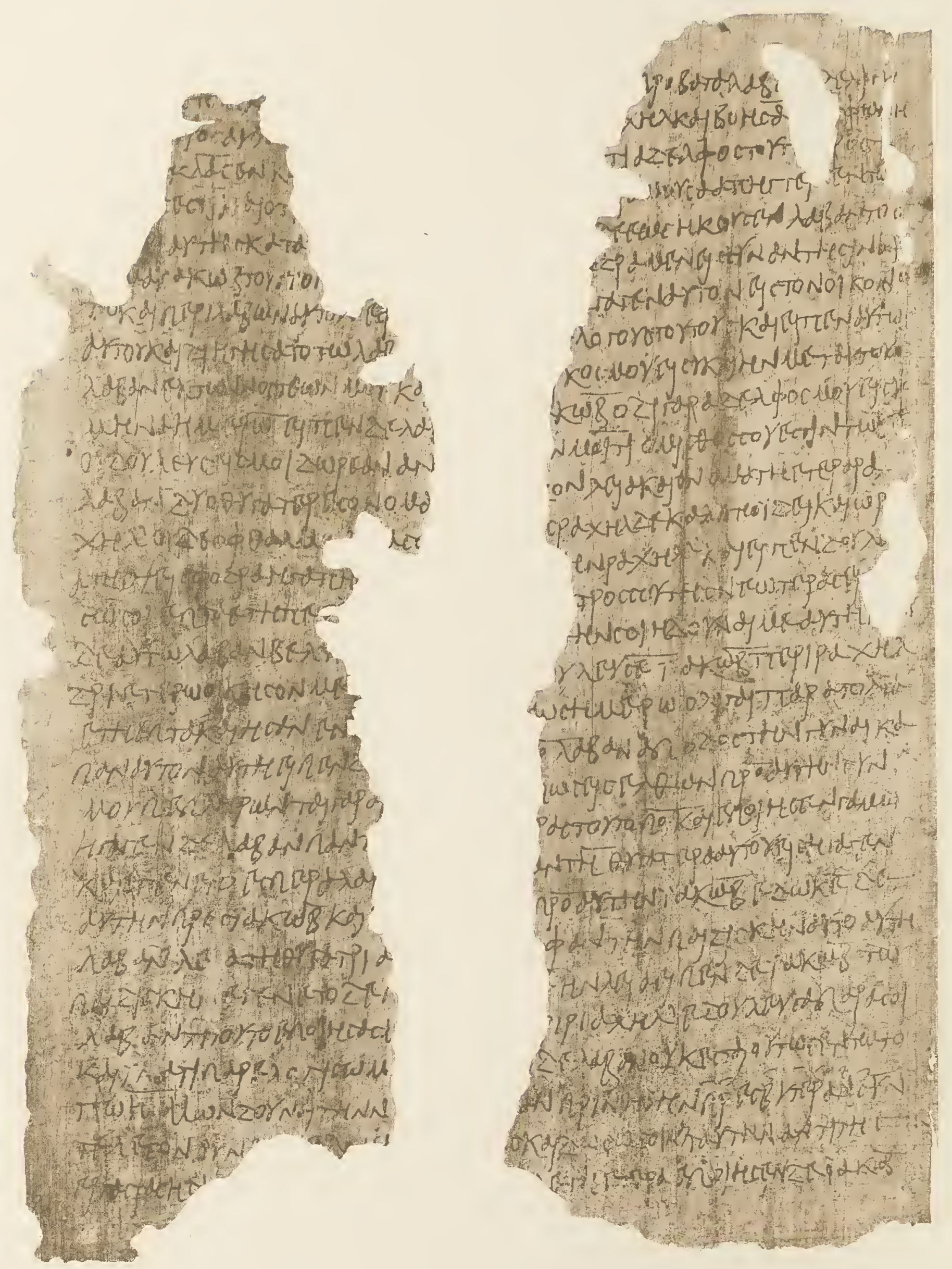

Genesis xxix, 10-28. 

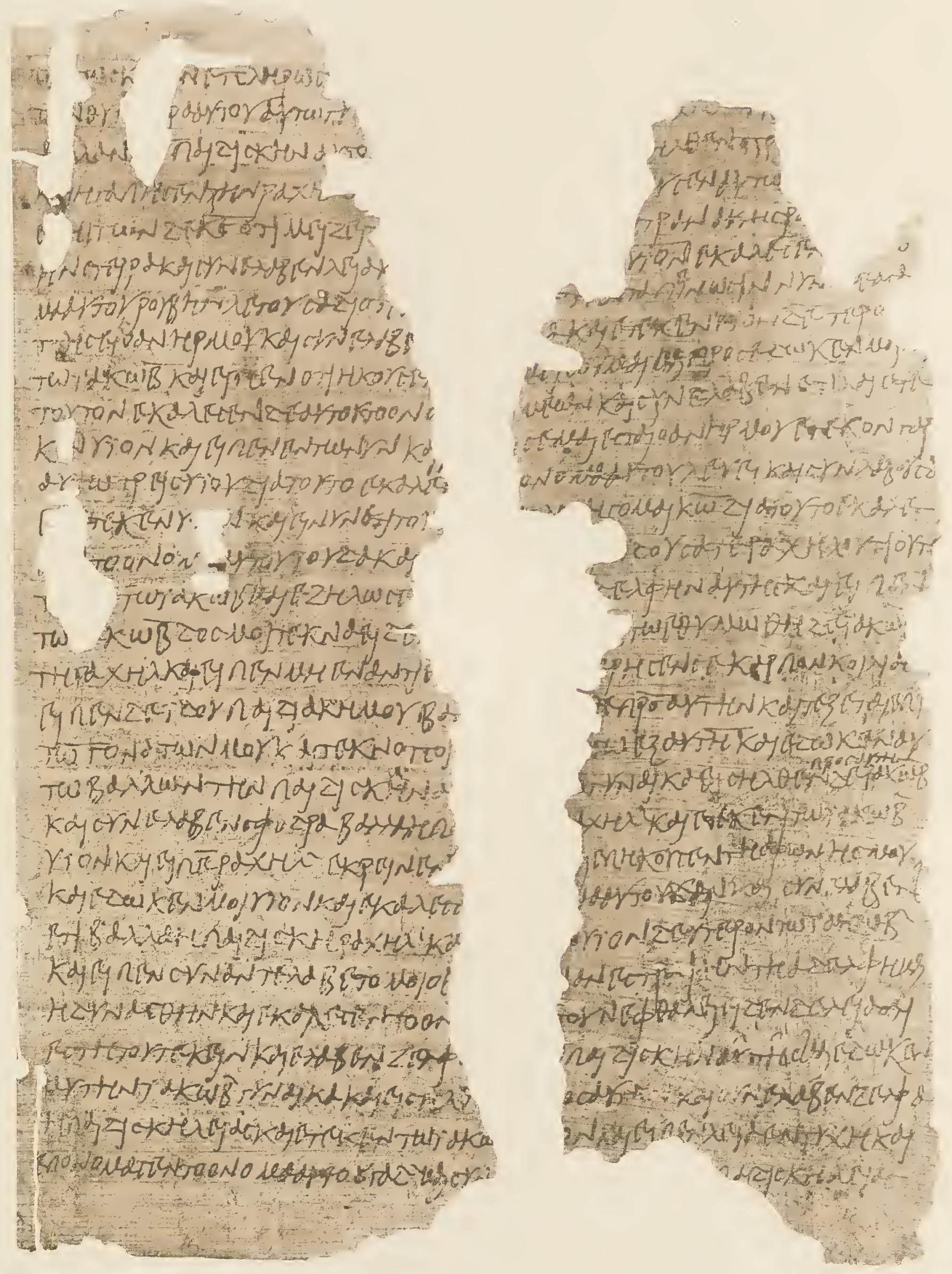

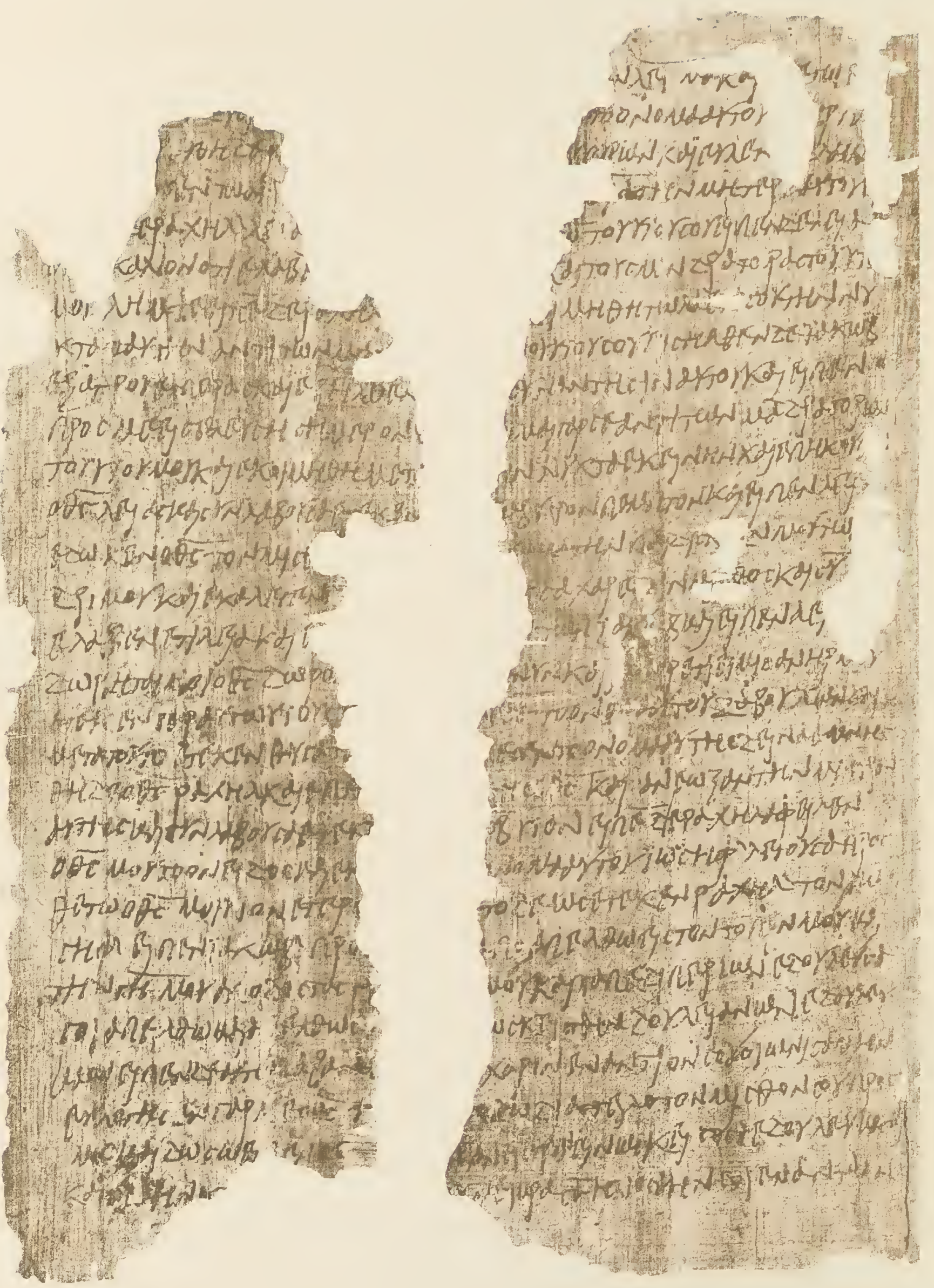

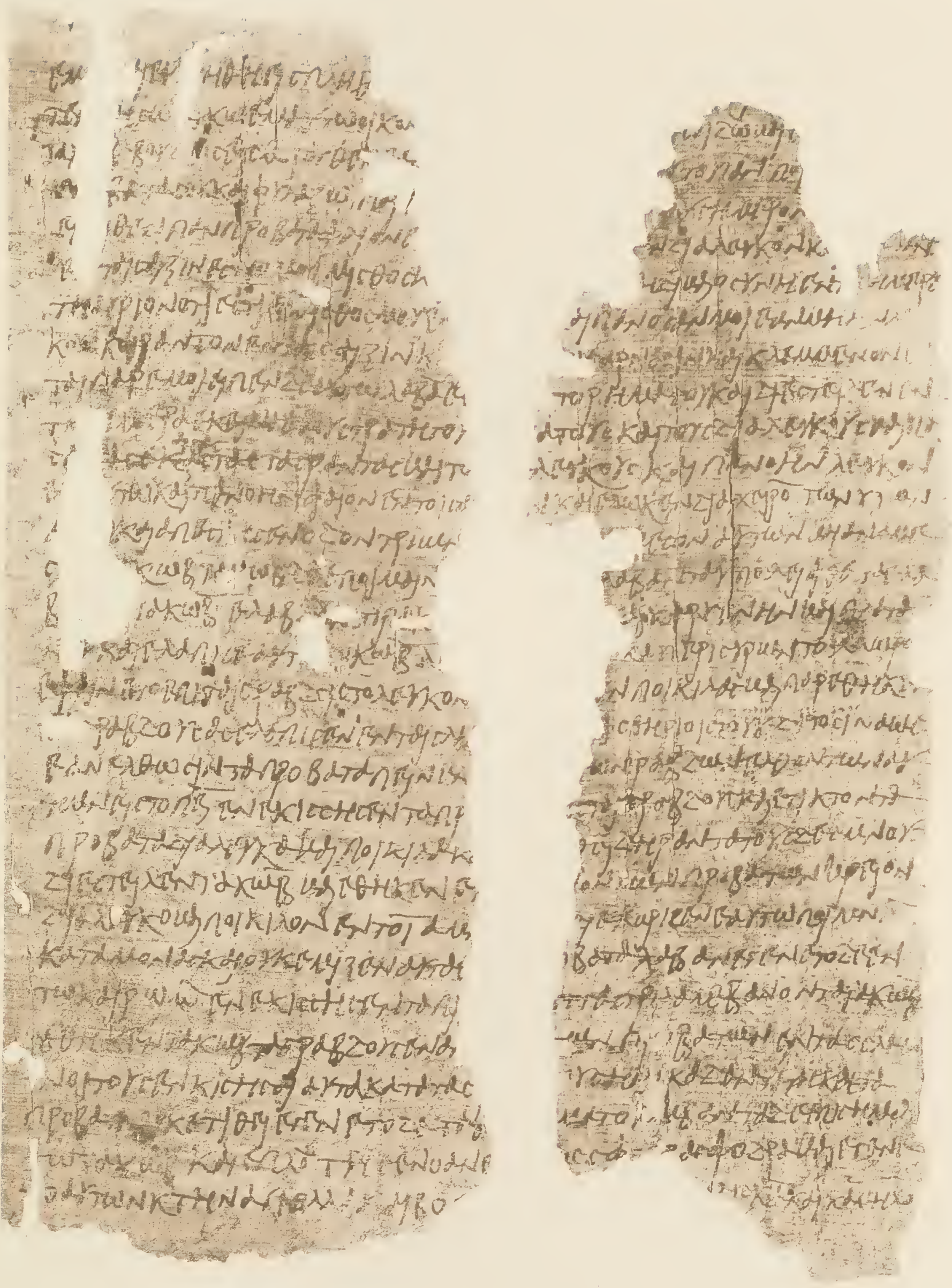

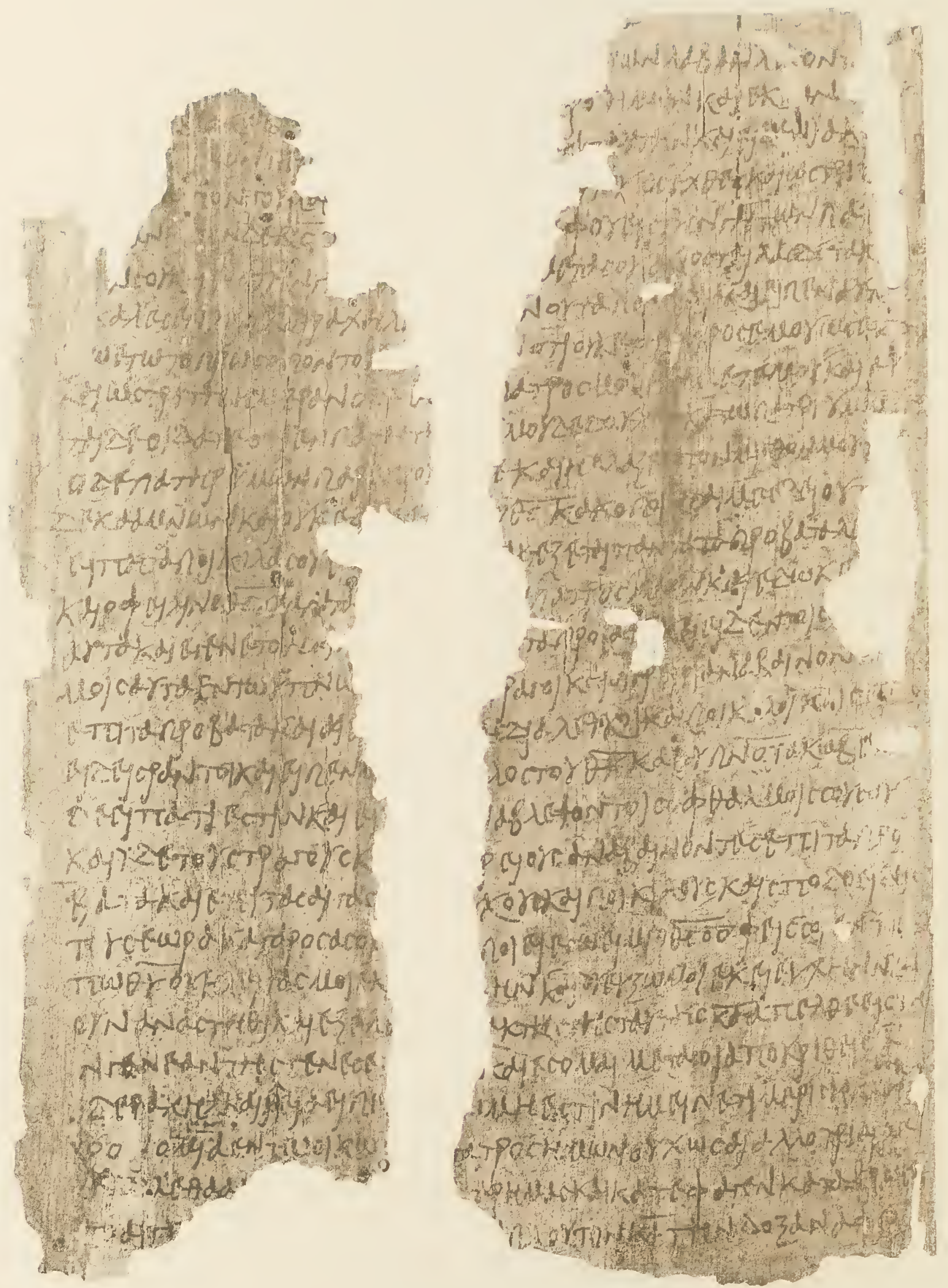

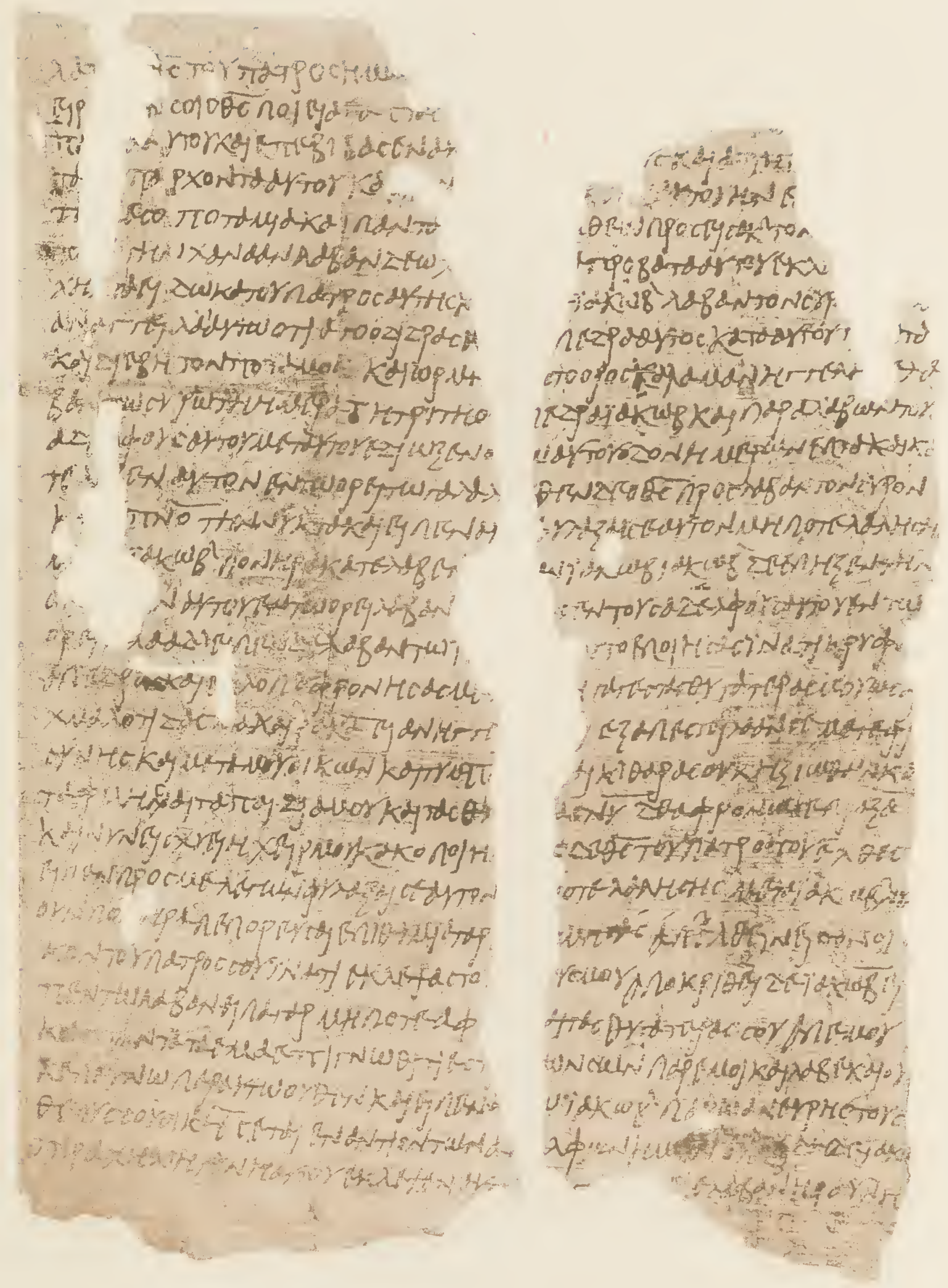


$$
\begin{aligned}
& \text { ch } 1 \text { ind }
\end{aligned}
$$

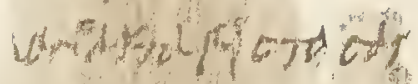

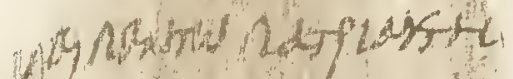

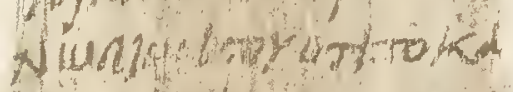

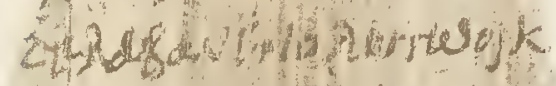

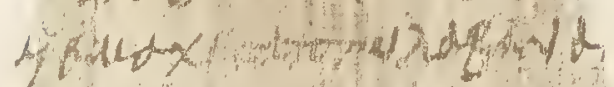

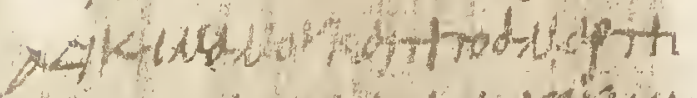

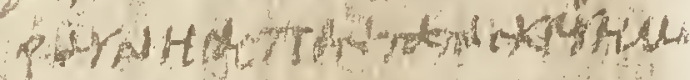

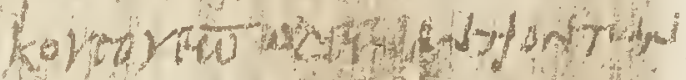

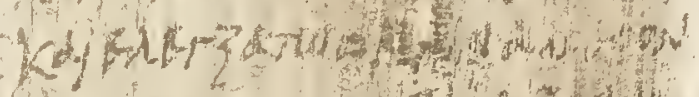

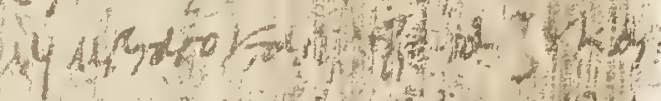

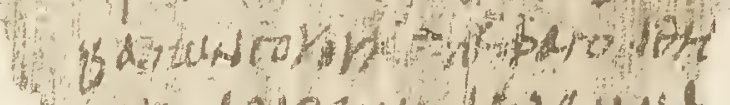

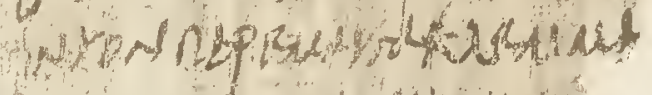

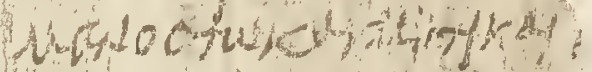

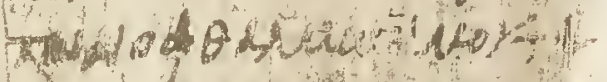

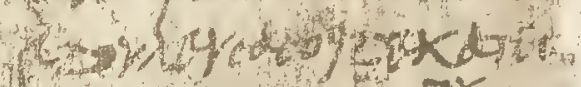

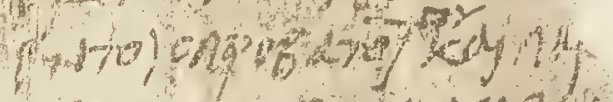

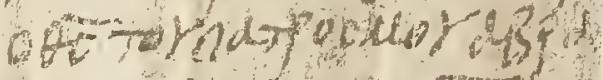

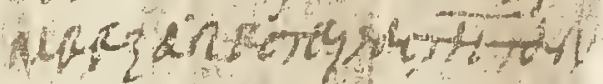

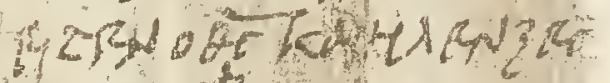

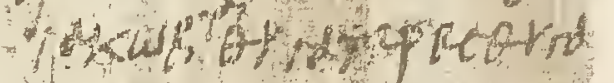

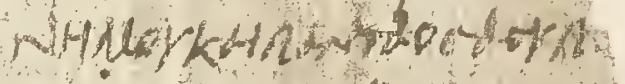

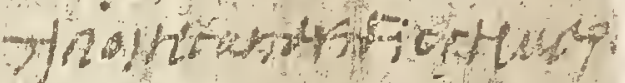

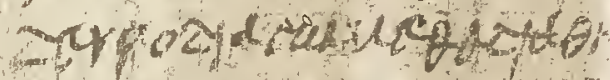

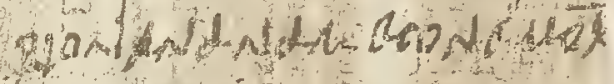

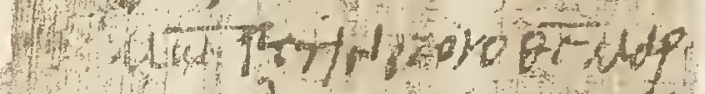

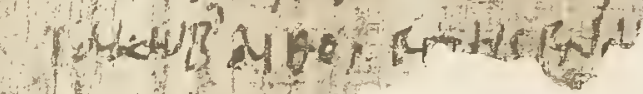

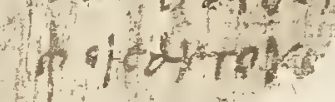

whyoyojkoy ford ky

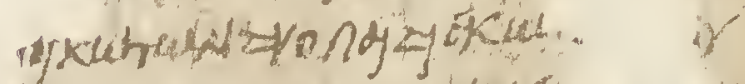

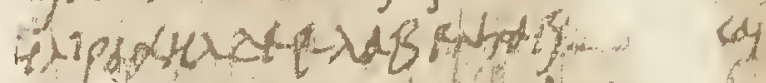
" YKonckedrond r.

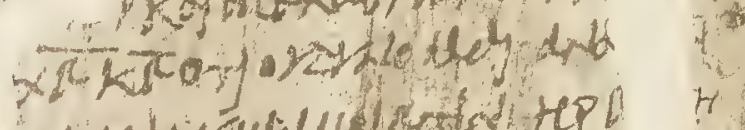

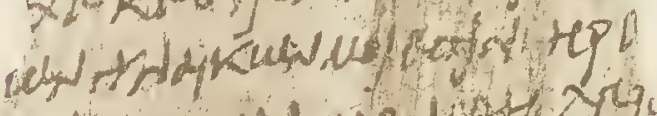

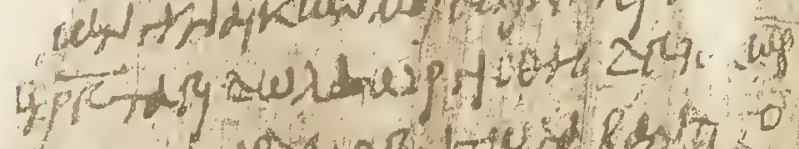

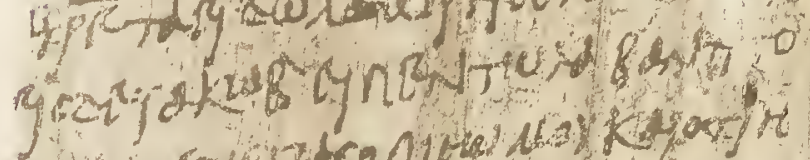

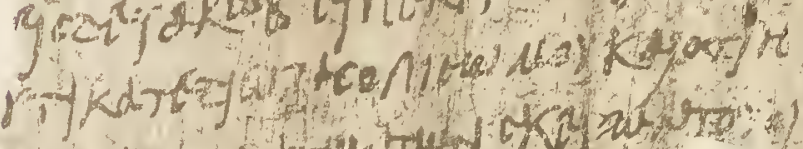

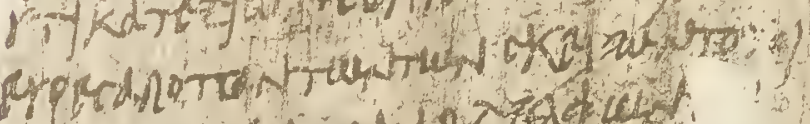

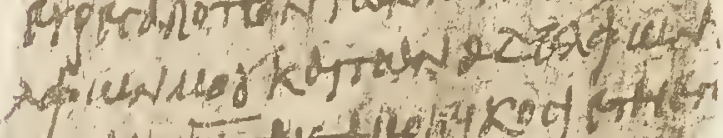

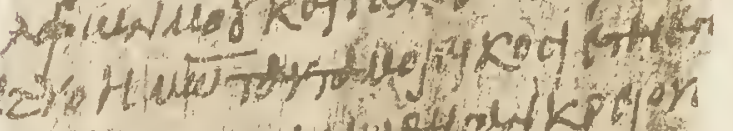

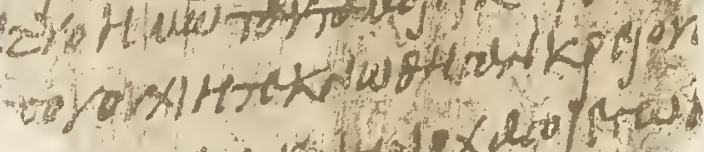

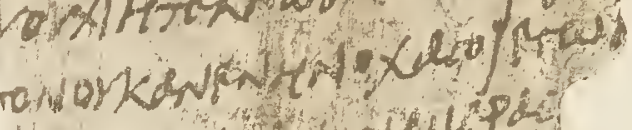

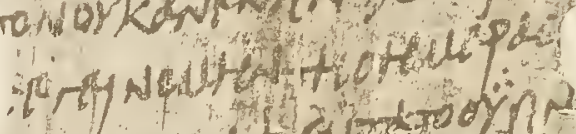

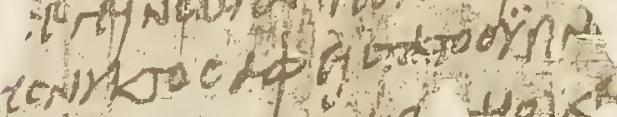

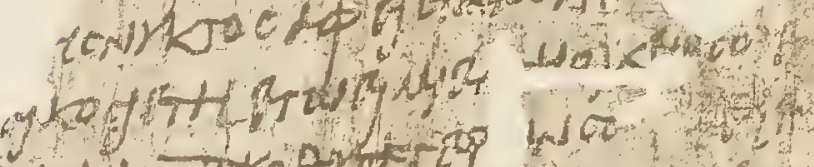

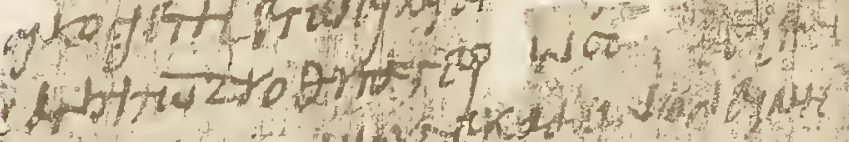

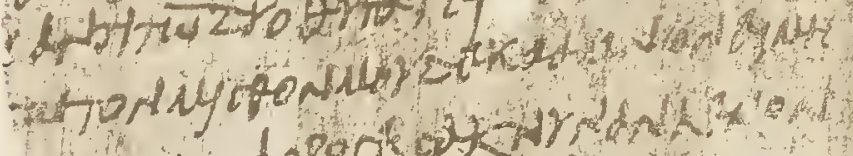

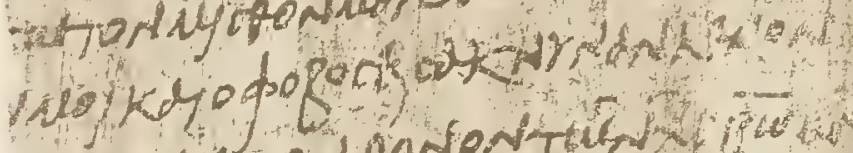

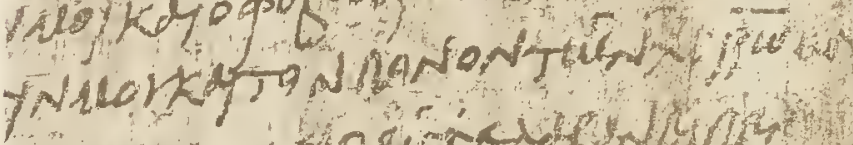

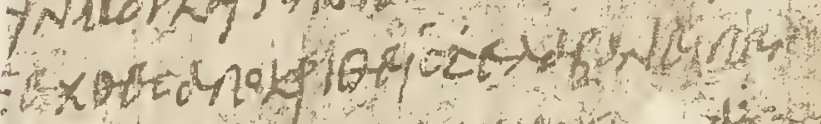

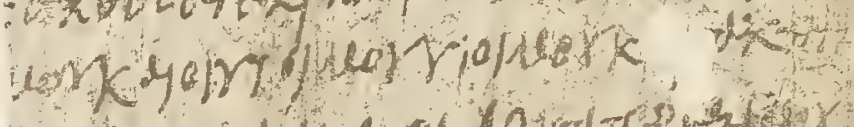

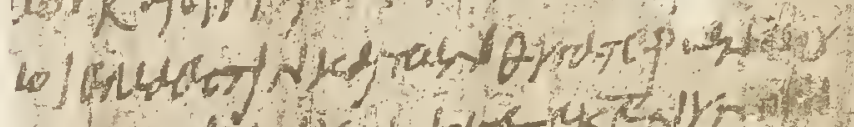

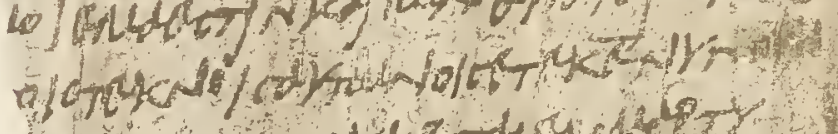

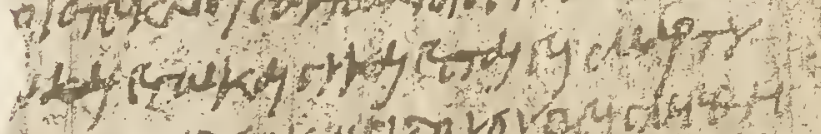

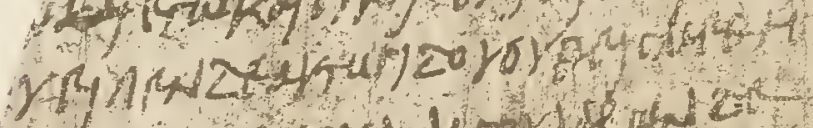

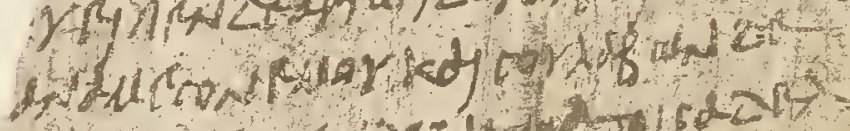

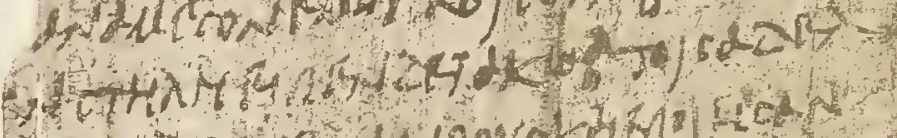

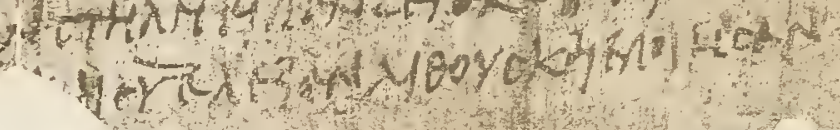




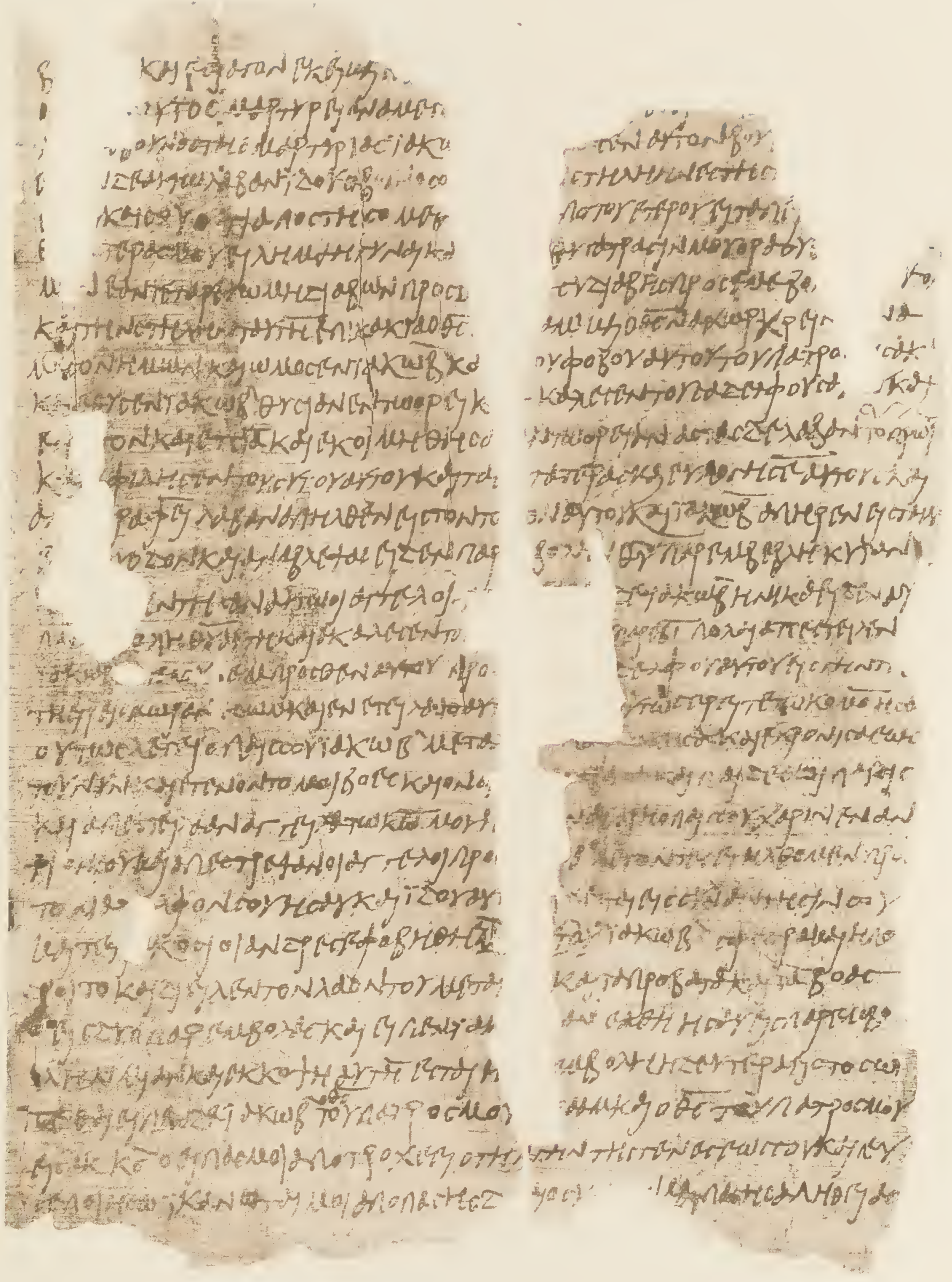



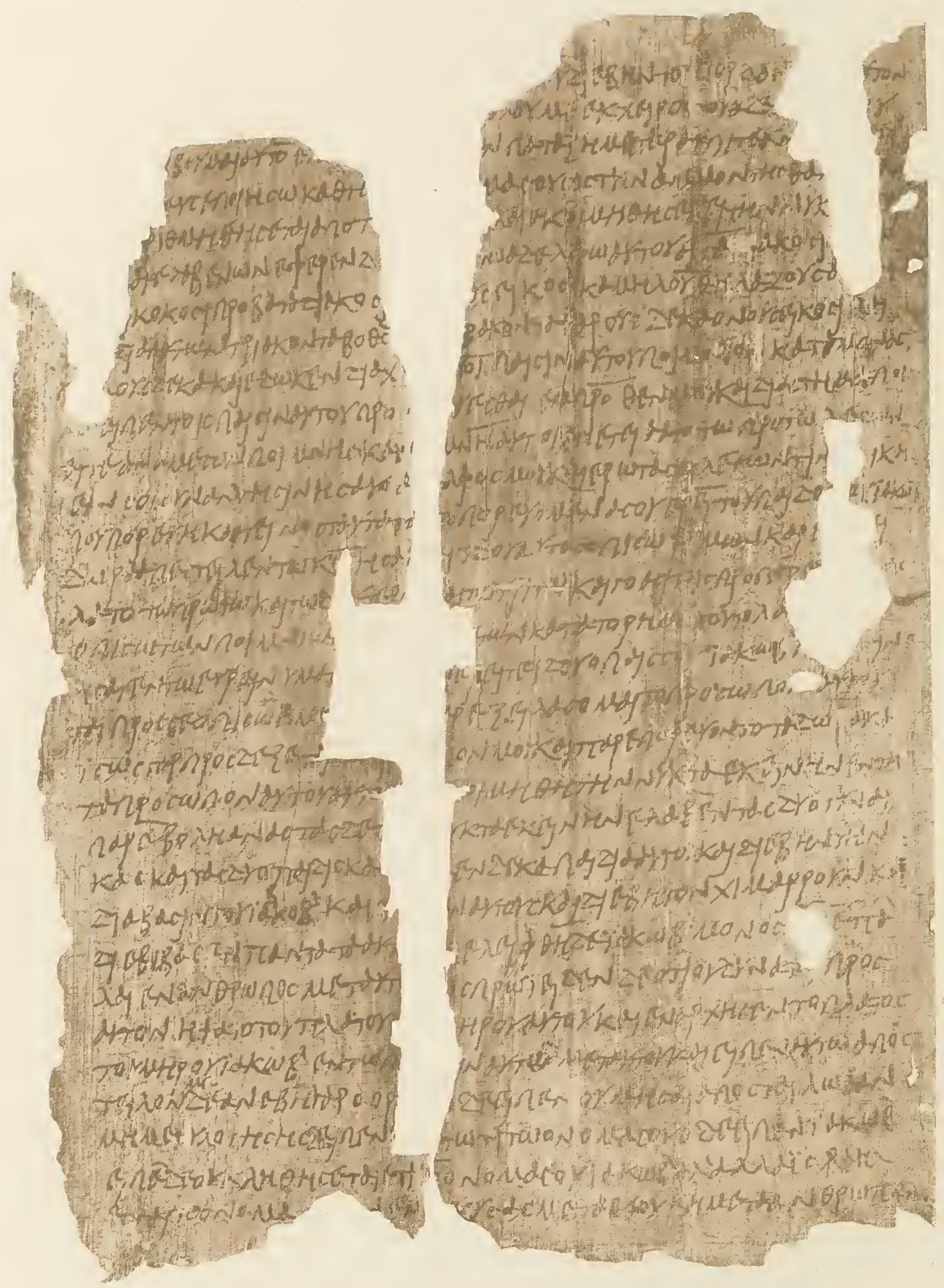


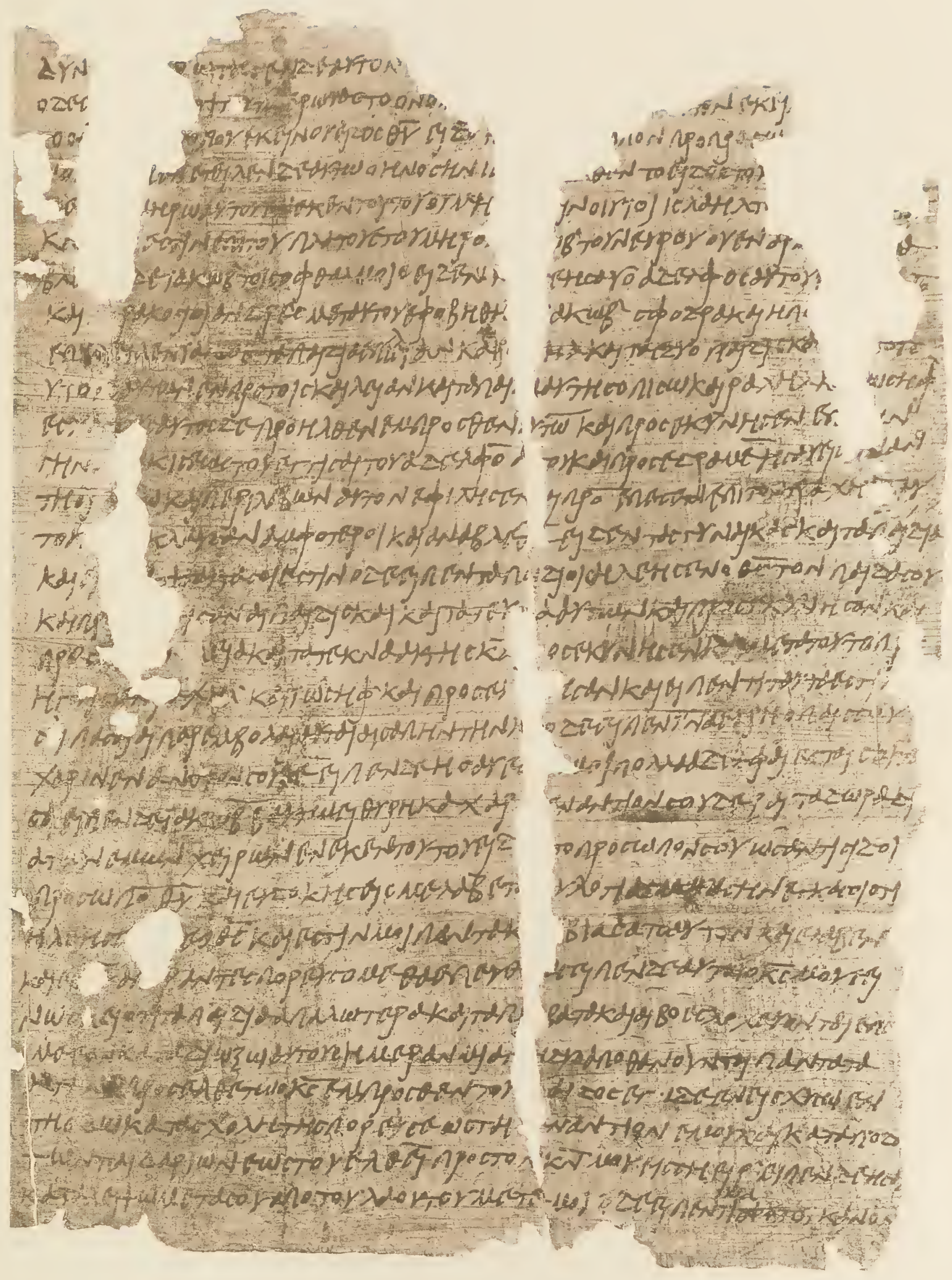


Hothenm

$42 x+15 \%$

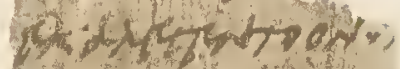

nexprokuknts

Syentortockyidy.

Hom 14 preos $5 x$

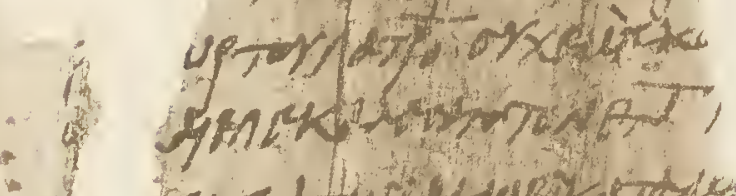

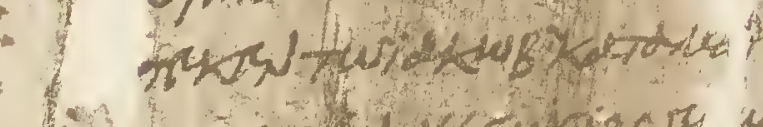

(6)

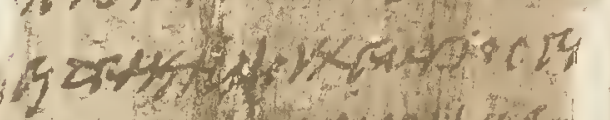

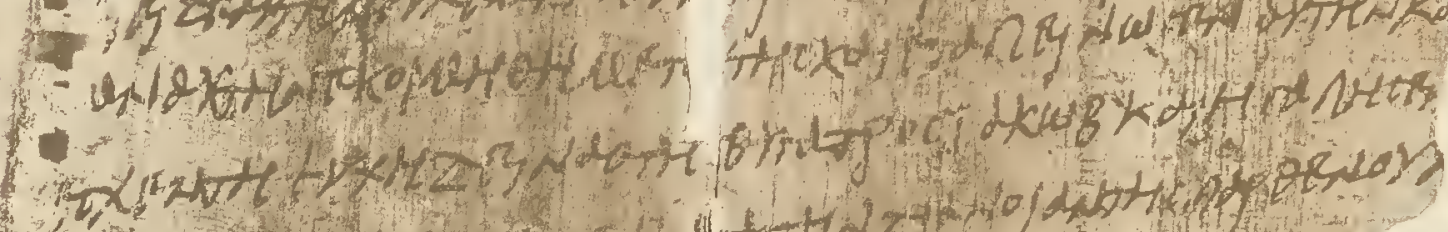

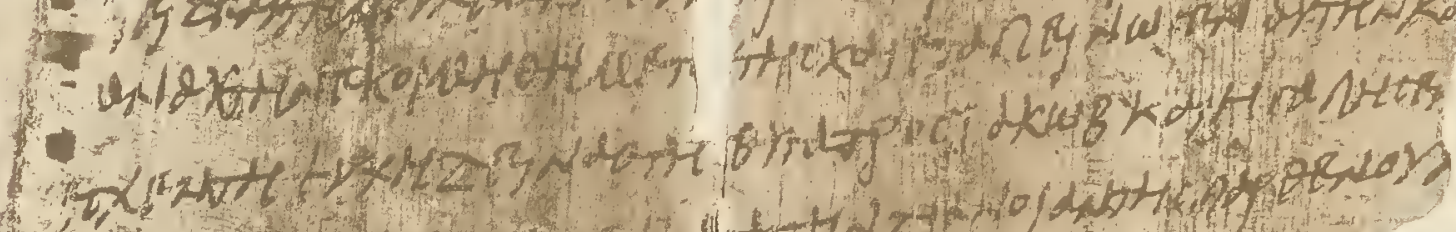

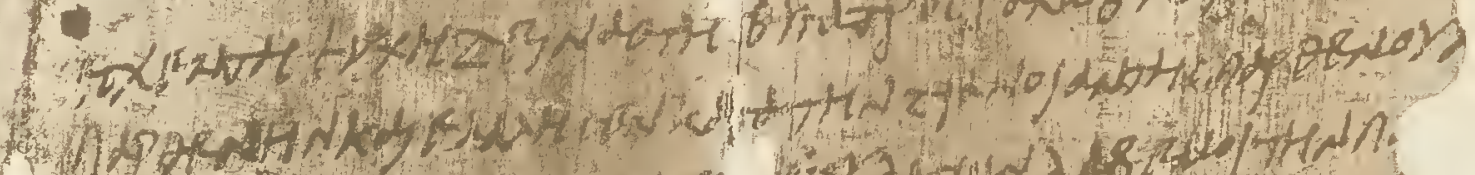

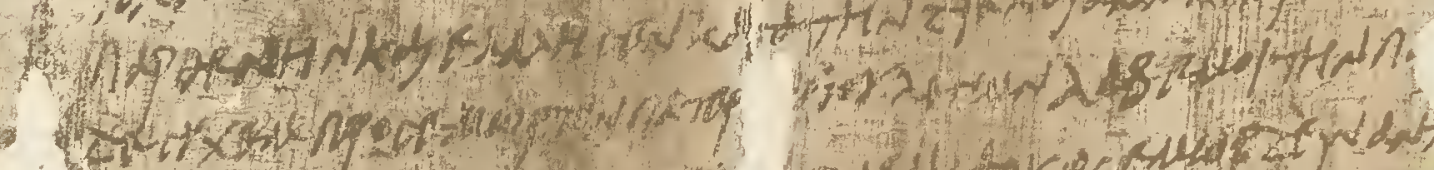

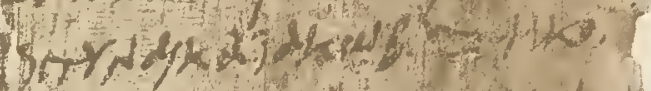

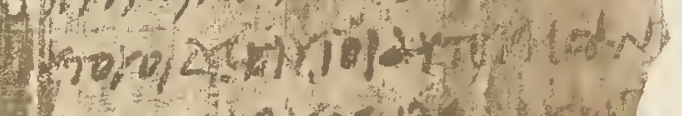

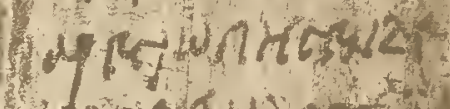

*ternatinge

Play-huttritor

the apd $=7 d x+1$

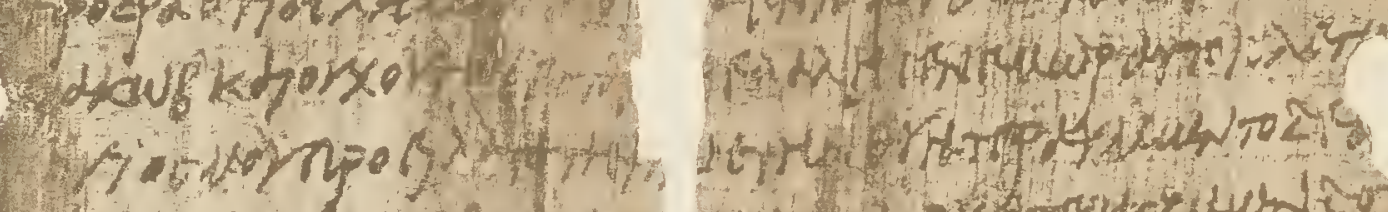

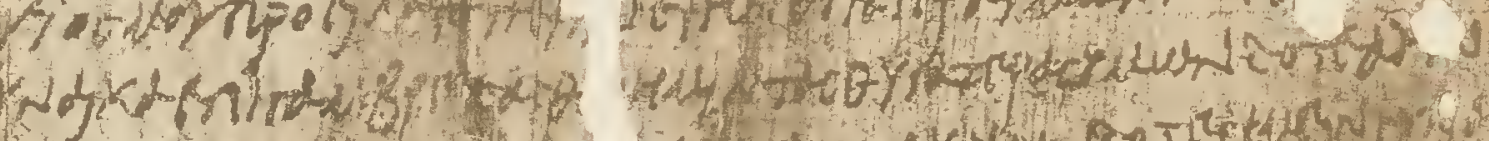

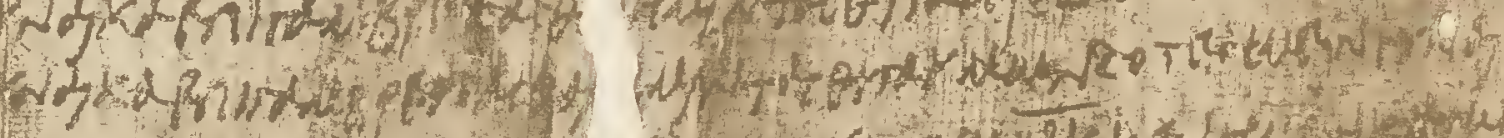

(1)

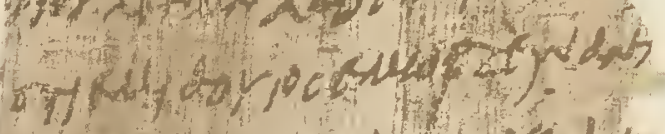

n-Tat

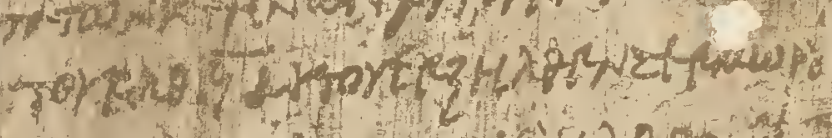

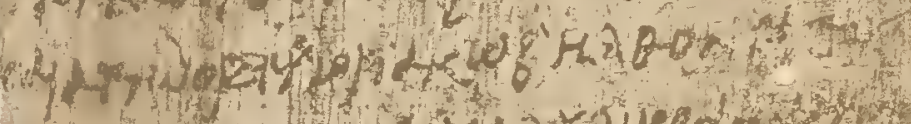

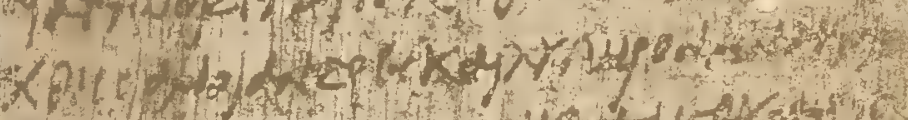

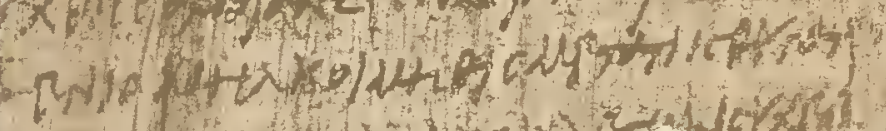

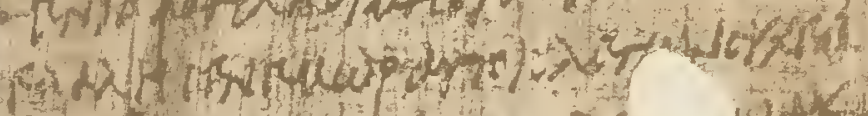

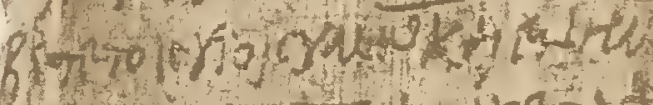

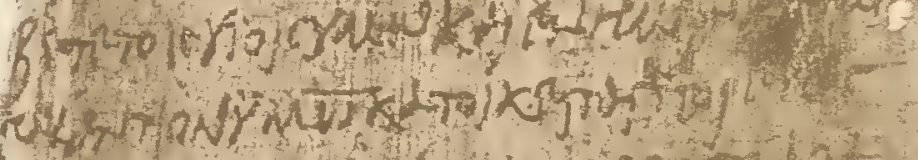

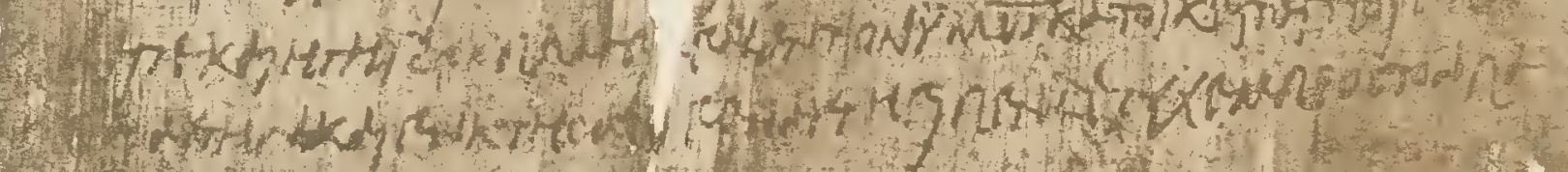

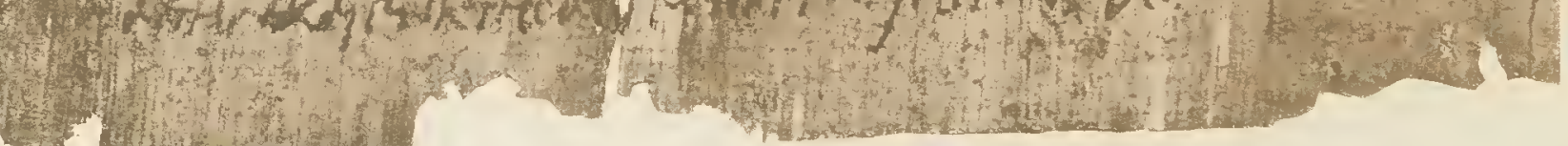




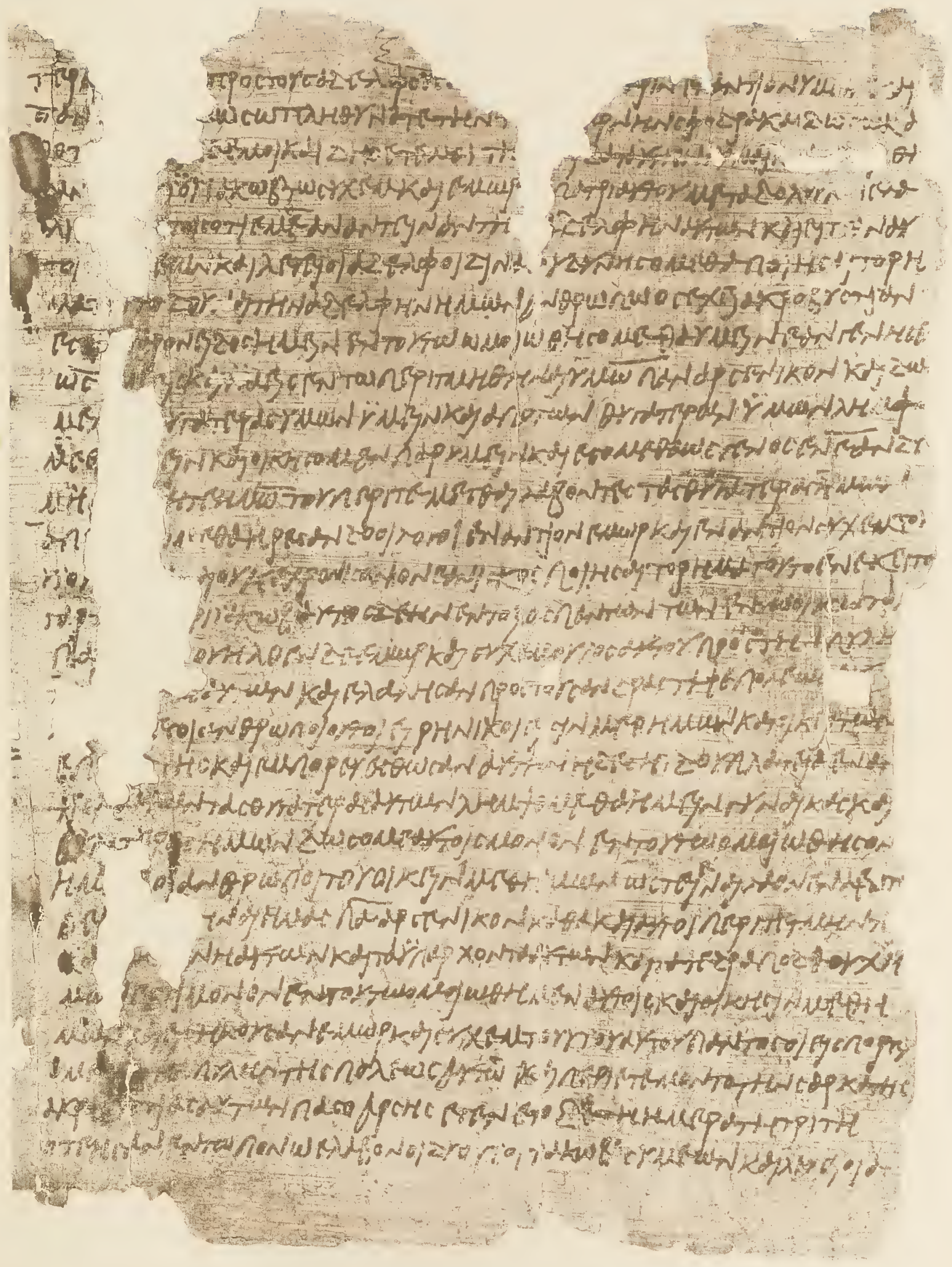



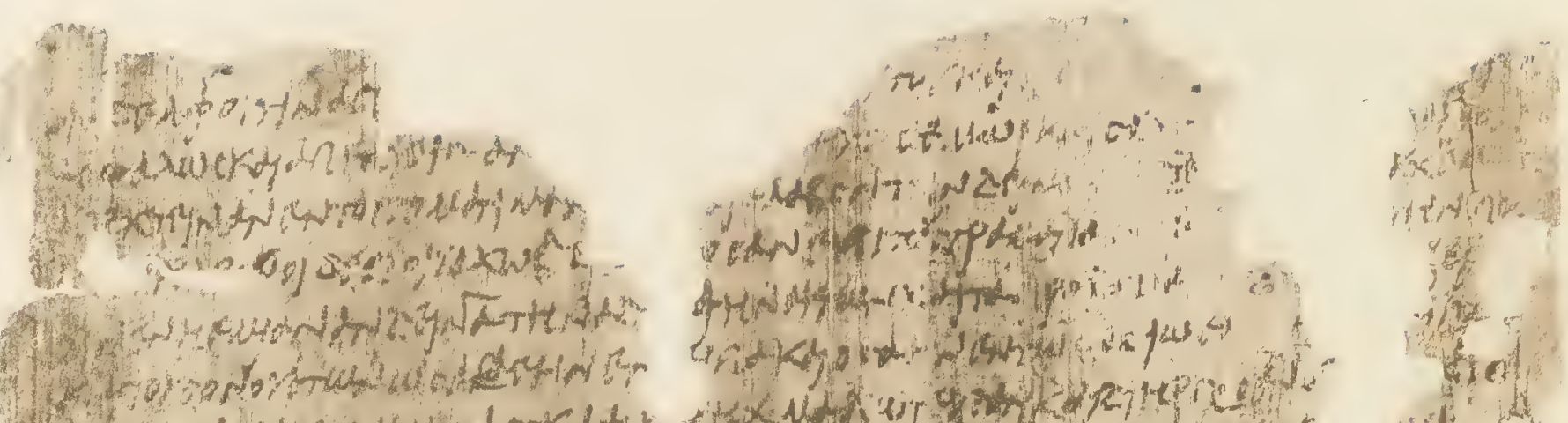

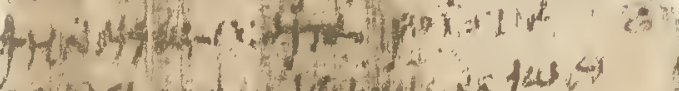

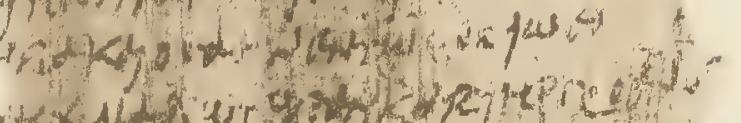

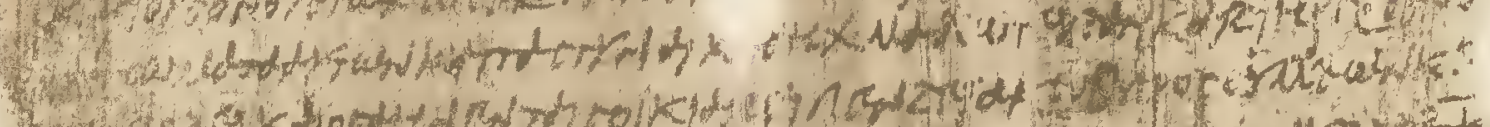

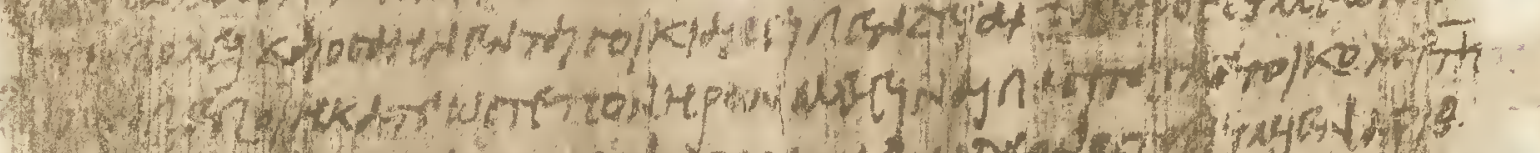

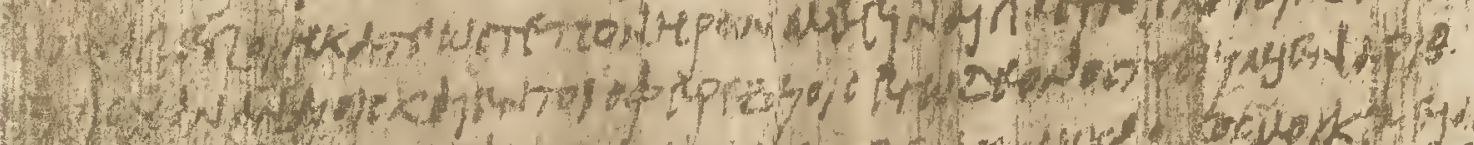

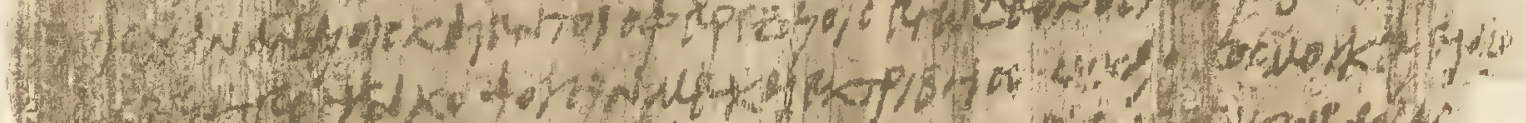

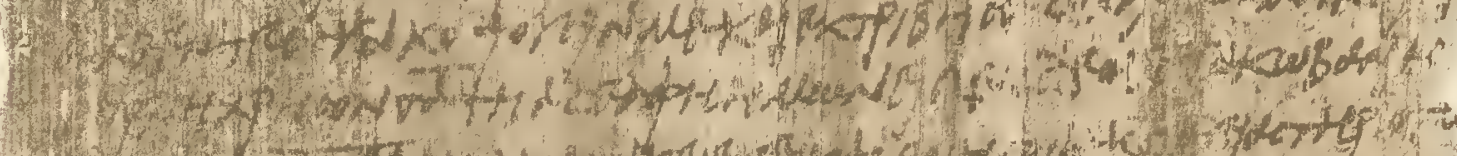

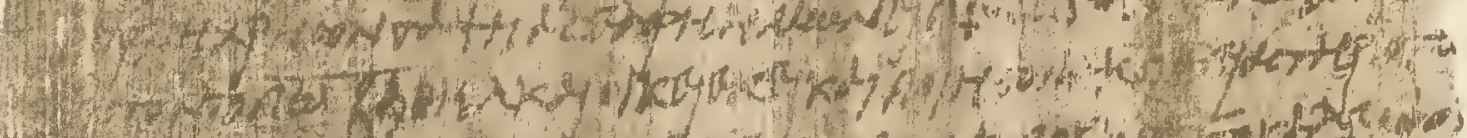

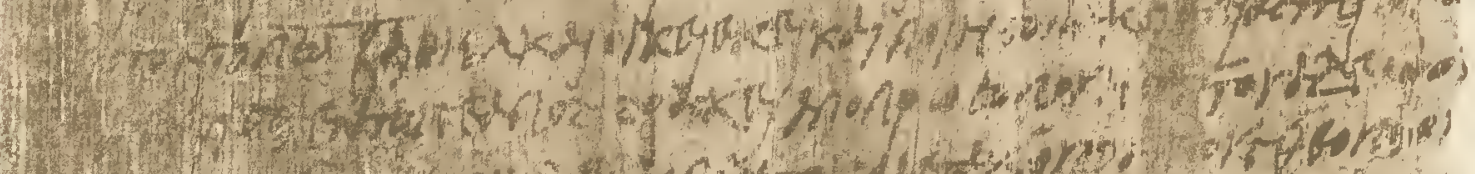

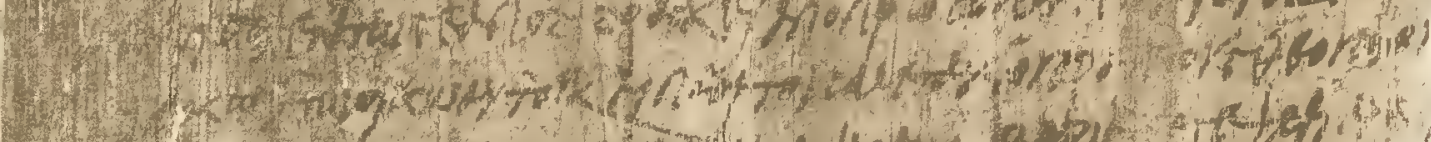

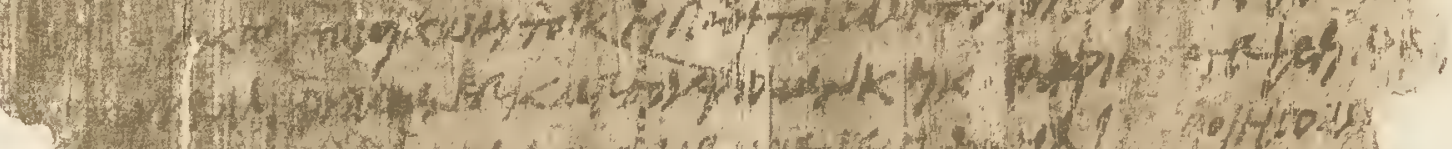

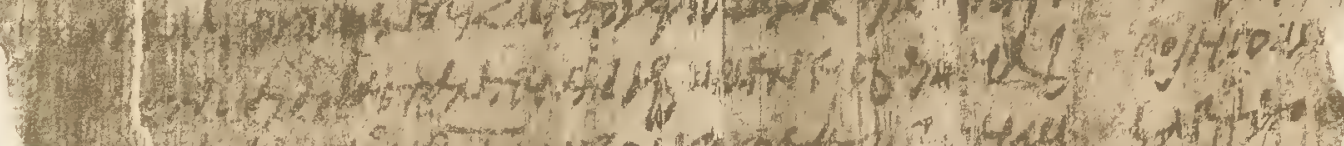

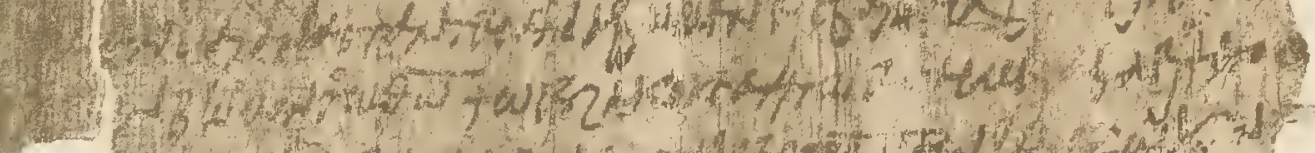

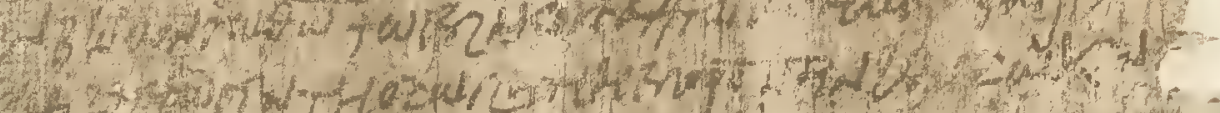
(n)

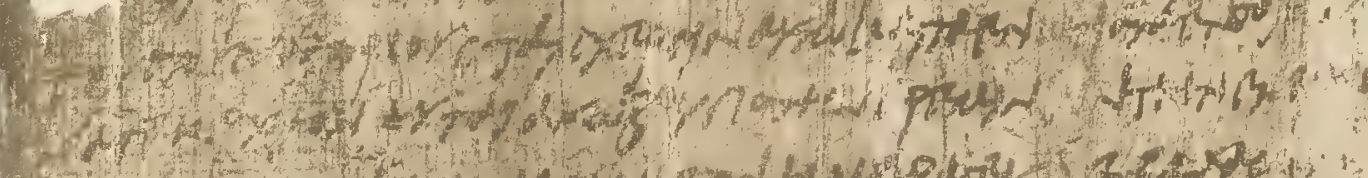

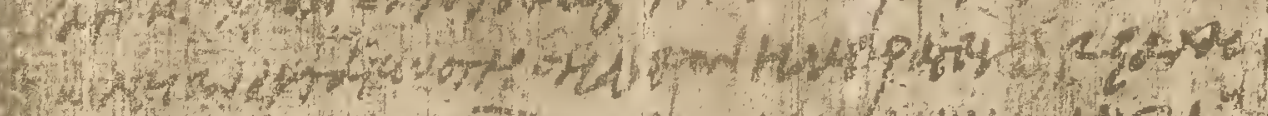

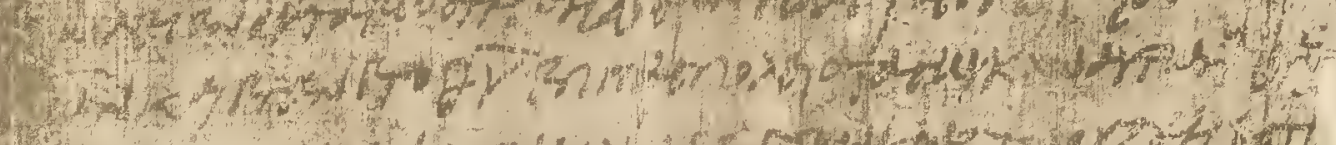

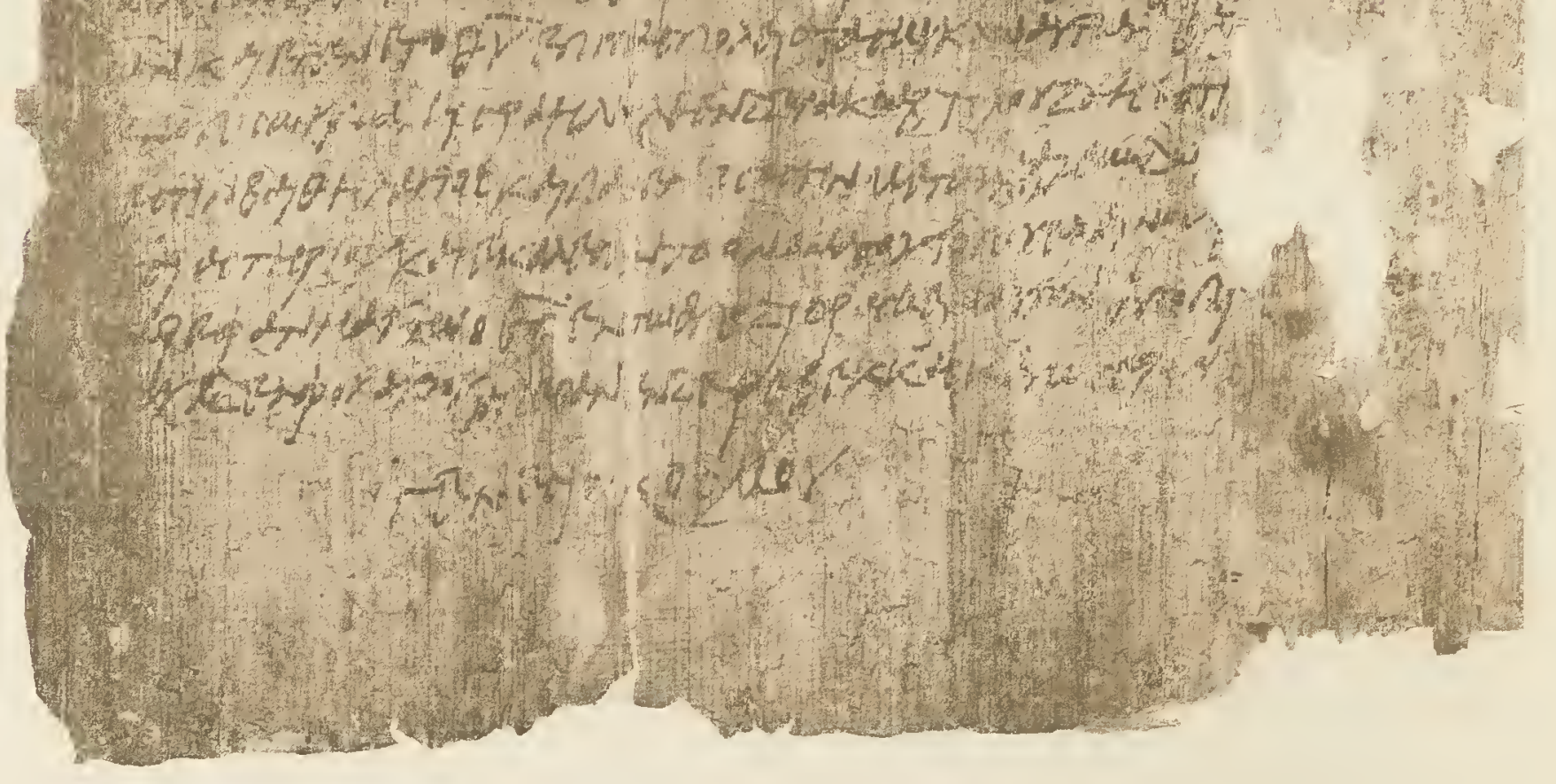












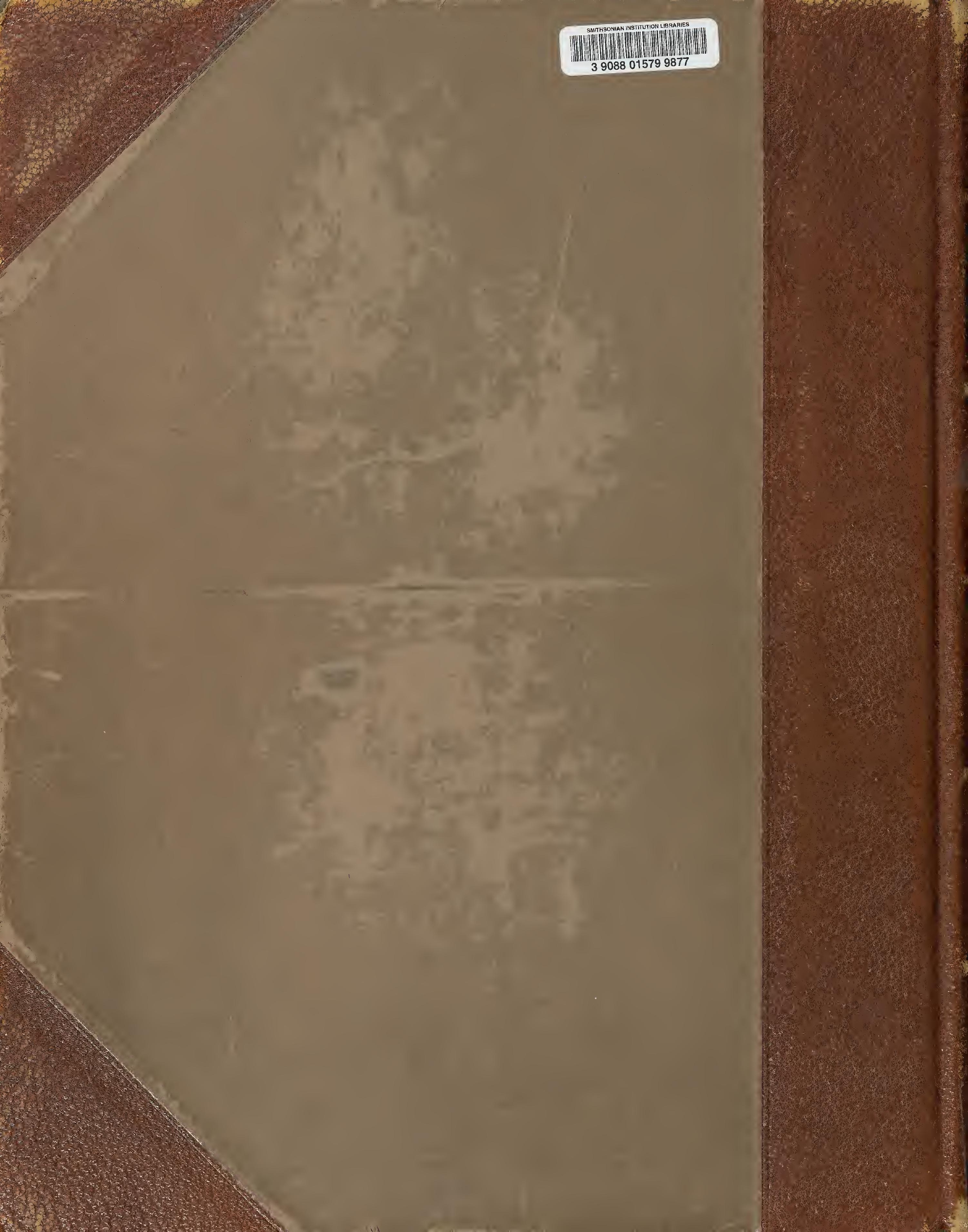

